\title{
Psychedelic bliss : memory and risk taking during MDMA intoxication
}

Citation for published version (APA):

Kuypers, K. P. C. (2007). Psychedelic bliss : memory and risk taking during MDMA intoxication. [Doctoral Thesis, Maastricht University]. Universiteit Maastricht. https://doi.org/10.26481/dis.20070405kk

Document status and date:

Published: 01/01/2007

DOI:

10.26481/dis.20070405kk

Document Version:

Publisher's PDF, also known as Version of record

\section{Please check the document version of this publication:}

- A submitted manuscript is the version of the article upon submission and before peer-review. There can be important differences between the submitted version and the official published version of record.

People interested in the research are advised to contact the author for the final version of the publication, or visit the DOI to the publisher's website.

- The final author version and the galley proof are versions of the publication after peer review.

- The final published version features the final layout of the paper including the volume, issue and page numbers.

Link to publication

\footnotetext{
General rights rights.

- You may freely distribute the URL identifying the publication in the public portal. please follow below link for the End User Agreement:

www.umlib.nl/taverne-license

Take down policy

If you believe that this document breaches copyright please contact us at:

repository@maastrichtuniversity.nl

providing details and we will investigate your claim.
}

Copyright and moral rights for the publications made accessible in the public portal are retained by the authors and/or other copyright owners and it is a condition of accessing publications that users recognise and abide by the legal requirements associated with these

- Users may download and print one copy of any publication from the public portal for the purpose of private study or research.

- You may not further distribute the material or use it for any profit-making activity or commercial gain

If the publication is distributed under the terms of Article $25 \mathrm{fa}$ of the Dutch Copyright Act, indicated by the "Taverne" license above, 


\section{Psychedelic bliss: Memory and Risk Taking during MDMA intoxication}

Kim Kuypers 
PSYCHEDELIC BLISS: MEMORY AND RISK TAKING DURING MDMA INTOXICATION 
(C) K.P.C. Kuypers, Maastricht, 2007

Cover image | Vital Kuypers

Print | Leën Offset Drukkerij NV Hasselt (Belgium)

ISBN/EAN | 978-90-9021656-0 


\title{
PSYCHEDELIC BLISS: MEMORY AND RISK TAKING DURING MDMA INTOXICATION
}

\author{
Proefschrift
}

Ter verkrijging van de graad van doctor aan de Universiteit Maastricht, op gezag van de Rector Magnificus, Prof. mr. G.P.M.F. Mols, volgens het besluit van het

College van Decanen, in het openbaar te verdedigen op donderdag 5 april 2007 om 12 uur.

door

Kim Paula Colette Kuypers

Geboren op 14 maart 1980 te Hasselt 


\section{Promotor}

Prof. dr. W.J. Riedel

\section{Co-Promotor}

Dr. J.G. Ramaekers

\section{Beoordelingscommissie}

Prof. dr. J.Jolles (voorzitter)

Prof. dr. V.Curran

Prof. dr. J.M.A. van Gerven

Dr. B. Jansma

Prof. dr. J. van Os

The studies described in chapters 2, 3, 6 and 8 were part of the IMMORTAL project, funded by the European Commission (Grant GMA1-2000-27043).

The research described in this dissertation was performed at the Maastricht Brain and Behaviour Institute, Department of Neurocognition, Faculty of Psychology, University of Maastricht.

Publication of this thesis was financially supported by: Servier Nederland Farma B.V., Wyeth Pharmaceuticals bv and the Dutch Ministry of Transport 
Paranimfen

Lies Moens

Marleen Wingen 



\section{CONTENTS}

CHAPTER 1.

General introduction ............................................................................................... 9

CHAPTER 2.

Transient memory impairment after acute dose of $75 \mathrm{mg} \mathrm{3,4}$

metbylenedioxymethamphetamine.

CHAPTER 3.

Acute dose of MDMA (75 mg) impairs spatial memory for location but leaves

contextual processing of visuospatial information unaffected.

CHAPTER 4.

Memory and mood during the night and in the morning after repeated evening doses of

MDMA.

CHAPTER 5.

Imaging the acute effects of a single dose of $M D M A$ on word learning. Preliminary

results.

CHAPTER 6.

Acute effects of 3,4 Methylenedioxymethamphetamine (MDMA) on behavioral measures of impulsivity: alone and in combination with alcohol.....

CHAPTER 7.

Acute effects of nocturnal doses of MDMA on measures of impulsivity and psychomotor

performance throughout the night.

CHAPTER 8.

$M D M A$ and alcohol effects, combined and alone, on objective and subjective measures of actual driving performance and psychomotor function.

CHAPTER 9.

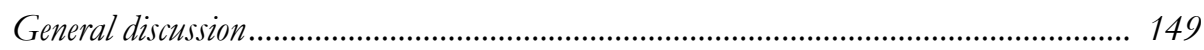

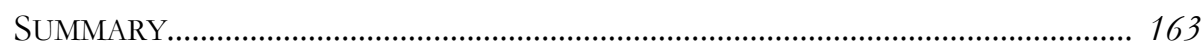

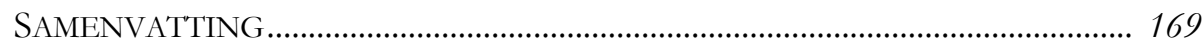

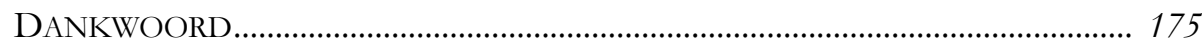

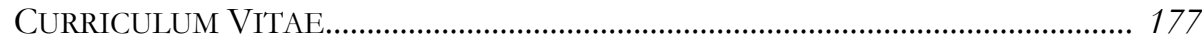

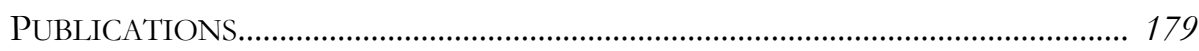





\section{CHAPTER 1}

\section{GENERAL INTRODUCTION}

\section{Background}

Ecstasy is a synthetic drug that is commonly used by people in the dance scene (ter Bogt \& Engels, 2005). It was in the late eighties that, along with the increasing number of large dance parties with loud rhythmic music, the so-called raves, its use became popular in Europe (Milroy, 1999). A recent report about the state of the drug problem in Europe presented estimations of the prevalence of current and life time ecstasy use in European countries in young adults (15-34 years). According to this report, the current ecstasy use is estimated to range between 0.6 $\%$ and $6 \%$ (the Netherlands: $3 \%$ ) and the life time prevalence ('ever used ecstasy') to range between $0.6 \%$ and $14 \%$ (the Netherlands: 7.5\%) (EWDD, 2005). Consequently, it can be stated that the volume of ecstasy use in society is considerable. Despite this fact, the occurence of cases with acute medical problems due to ecstasy is rare (Hall \& Henry, 2006). Concern however is raised by reports of possible long term effects in ecstasy users such as psychiatric disorders and cognitive disturbances e.g. memory problems (Parrott, 2001; Parrott, Milani, Parmar, \& Turner, 2001; Soar, Turner, \& Parrott, 2001). If it is true that ecstasy causes long-lasting memory problems, these subjects might be the early dementia cases of the future. Beside these possible long term threats, acute behavioral effects could interfere with daily life functions i.e. driving a car. In a number of impaired drivers, MDMA concentrations were detected in blood (Logan \& Couper, 2001, 2003). In addition, comparative studies have suggested that ecstasy users display more aberrant impulse control and decision making compared with (poly-)drug users or drug naïve persons (Butler \& Montgomery, 2004; Moeller et al., 2002; Morgan, Impallomeni, Pirona, \& Rogers, 2006). When ecstasy is the cause of these deviant behaviors, it is reasonable to hypothesize that ecstasy could also acutely affect impulse control and decision making and consequently hamper daily life activities. The present thesis describes a range of studies that were designed to assess the effect of MDMA on memory, impulsivity, and psychomotor performance during intoxication. The major aim was to establish a quantitative and causal association between MDMA exposure and performance impairment. 


\section{Ecstasy, behavior, and the brain}

When observing patterns of ecstasy use, it is shown that the majority of the dance attendees take one or two pills on an evening. The timing of intake can differ between individuals. Some ecstasy users take several pills simultaneously (stacking); others take a second pill when the desired effect is wearing off (boosting). A common practice is the combined use of ecstasy with other substances such as cannabis, alcohol, and amphetamines (Barrett, Gross, Garand, \& Pihl, 2005; Topp, Hando, Dillon, Roche, \& Solowij, 1999; Winstock, Griffiths, \& Stewart, 2001).

The major psychoactive constituent of ecstasy is $\pm 3,4$ methylenedioxymethamphetamine or short, MDMA. According to the annual report (2005) of the 'Drugs information and monitoring system' (DIMS) of the Netherlands, an ecstasy pill contains on average $75 \mathrm{mg}$ of MDMA. The doses range from a few milligrams to $200 \mathrm{mg}$ (DIMS, 2005). Peak plasma concentrations are reached approximately 2 hours after intake. The subjective effects begin approximately 90 minutes after intake and they can last up to 4 to 6 hours (Hall \& Henry, 2006; Kalant, 2001).

Shortly following MDMA ingestion, subjective effects can be experienced i.e. mood lifting, stimulant and entactogenic effects (Baylen \& Rosenberg, 2006; de la Torre et al., 2004; ter Bogt \& Engels, 2005). Concretely this means that euphoria, increased energy and an intensification of impressions (e.g. music, light, contact with people) are being experienced (ter Bogt \& Engels, 2005). It is this combination of effects that characterizes ecstasy and distinguishes it from other substances like amphetamines or LSD which have only stimulant or hallucinogenic effects respectively (Kalant, 2001). This mix of mood lifting, stimulant and entactogene effects was also reported by the subjects that participated in the studies presented in this dissertation. Here are some examples of subject reports:

- 'While using XTC, I feel energetic, good-bumored, happy, and euphoric but most of all good. I only use XTC on house-parties because there the drug comes to its right. The combination of XTC, music and the being together with like-minded people makes the experience a special one. XTC tanght me how easy it is to communicate with other people even (and also) when you are not using it."

- "Blissful, pleasant, self conscious, friendly, a little philosophical"

- "Good, love, pleasant, warm, free, energetic, dance"

- "It resembles the feeling of being in love but then multiplied by ten."

- "Cheerful, secure, generally good" 
- "Euphoric, a lot of things are beautiful, more in particular music and light, feeling of closeness to others (friends)"

- "Pleasant feeling; you want to dance and you love everybody"

- "You feel just happy, energetic and clearbeaded; XTC gives you the feeling that you can take on the world"

- "When I use ecstasy, all my inhibitions subside: I'm more relaxed, the music sounds great and the need to dance increases"

- "It gives a nice tingling feeling from the stomach, everybody likes you and you talk nineteen to the dozen. You are more relaxed in your contact with people, the music sounds even better than normal, in fact, it intensifies your feelings and you experience such an evening at a party more intense than when you are not using. The perception of time however is totally gone. Often, an evening has past and you have the feeling that you were just 2 hours there, but instead you have been dancing and talking for 8 hours"

- "When on ecstasy, I feel good and more awake than when I use alcohol. It is just like entering another world where everything is different, prettier and brighter."

- "I feel energetic and ooze of confidence. The contact with people is easier because I adopt an open attitude."

- "I get extra energy from ecstasy. I can dance till the early morning."

Research into the motives to use ecstasy have led to the conclusion that essentially the experience of the expected enhancing effects i.e. euphoria and energy, is the main drive (ter Bogt \& Engels, 2005). This was also confirmed by the reactions of the participants in our studies when they were asked to describe the reasons why they use ecstasy. Here are some examples of subject reports:

- "I use ecstasy because I like the feeling and because it enables you to dance longer on a party"

- "Why not? The feeling you get is great..."

- "I use ecstasy for the psychoactive effects, to put daily things behind me for a while and to do some introspection"

- "It gives a surplus when attending dance parties, everything is more intense"

- 'I use ecstasy because it gives you a pleasant feeling and releases you from all your inhibitions. You don't experience the feeling of becoming tired after having danced the whole night long"

- "With the belp of ecstasy I can party for hours on end"

- "I use ecstasy to enjoy myself, to relax. I experience the music more intense and feel more confident and free. Another reason is that it helps in keeping me going" 
In addition to the positive effects, ecstasy use is also accompanied by short-term side effects occurring early, with respect to administration, and later on. Early short time side effects i.e. fatigue, lack of energy and difficulty concentrating, arise approximately 24 hours after MDMA ingestion. Late short-term side effects occur up to 7 days post-drug and are more or less comparable to early short time effects with the exception that they last longer (de la Torre et al., 2004).

MDMA produces these positive and negative effects through the interaction with several neurotransmitter systems such as the serotonergic (5-HT), dopaminergic, noradrenergic, cholinergic and histaminergic systems. The main effect of MDMA is on the serotonergic system where it acts as a powerful indirect 5-HT agonist. MDMA causes an increased release of 5-HT, a blockade of the 5HT reuptake mechanism, and inhibition of tryptophan hydroxylase, the precursor involved in the synthesis of 5-HT (Britt \& McCance Katz, 2005; Schmidt \& Kehne, 1990). Animal studies have shown that following ingestion of MDMA, there are two depletion phases of 5-HT. The increased release of 5-HT combined with the absence of reuptake and synthesis causes the 5-HT to be depleted in the first 3 to 6 hours after administration of MDMA. This first phase is depicted as the acute and reversible phase as it disappears within 12 hours, when the levels of 5HT have returned to normal. The second depletion phase arises between 24 hours and 7 days post-drug, and is called the long-term or neurotoxic phase. This is because this second phase can be accompanied by the loss of 5-HT uptake sites, an indication of neurotoxic damage (Green, Mechan, Elliott, O'Shea, \& Colado, 2003; Schmidt \& Kehne, 1990). This persistent damage to neurons has been shown to occur in animals when administrated MDMA following a repeated high dosing scheme (Ricaurte, Yuan, \& McCann, 2000). Whether it is justified to extrapolate these data to humans is however a topic of debate because of differences between animal studies and humans e.g. dosage, and the route of administration (De La Torre \& Farre, 2004; Rattray, 1991). An indication for the second 5-HT depletion phase in humans is the existence of lowered mood, a few days after the use of ecstasy, or the so called 'mid-week depression', (Curran \& Travill, 1997). Furthermore, there are also imaging studies showing signs of structural changes in heavy users e.g. lower 5-HT transporter densities, although these changes are possibly temporary (Green et al., 2003; Reneman, de Win, van den Brink, Booij, \& den Heeten, 2006).

Serotonin is a widespread neurotransmitter that is involved in diverse physiologic and cognitive processes. An example of a cognitive function in which 5-HT is implicated is memory performance. In several studies it has been shown 
that disruption of the normal 5-HT levels results in cognitive deficits (Meeter, Talamini, Schmitt, \& Riedel, 2006).

\section{Ecstasy and memory}

Ecstasy use has regularly been associated with cognitive problems in abstinent and/ or current users. The most prominent deficit linked with ecstasy use is impaired memory performance i.e. even in users who had been abstinent for 2.5 years, memory problems were still observed (Green et al., 2003; Parrott, 2001; Thomasius et al., 2006).

The aforementioned authors, probably encouraged by knowledge from animal experiments that showed ecstasy to cause serotonergic neurotoxicity, tend to conclude that ecstasy use causes long-lasting memory deficits. None of the studies that demonstrated an association between ecstasy use and memory impairment were true experiments, which constitute the main problem why the causality of the observed association can not be assessed with any degree of certainty.

The studies investigating the long-term effects of ecstasy use typically retrospectively compare between groups the cognitive performance of abstinent and/or current ecstasy users with that of poly-drug users or in some cases with that of cannabis users. However, these types of studies offer no good solution when trying to uncover the source of the cognitive problems observed in ecstasy users. Several confounding factors complicate these retrospective between group comparisons. The memory deficits could be the result of other factors related with the ecstasy use i.e. a particular lifestyle characterized by a disturbed eating and sleeping pattern or poly-drug use. Because of these confounding variables it is not possible to draw a conclusion about the causality of the association between ecstasy use, and memory impairment; more methodological scrutiny is required to achieve this (Gouzoulis Mayfrank \& Daumann, 2006). Halpern and colleagues (2004) tried to control for these confounding variables by using a group of pure MDMA users (who used little else), and comparing their performance with that of a group of subjects who had a comparable lifestyle, save for MDMA use. When splitting the MDMA group into a moderate and a heavy user group, it appeared that heavy users performed worse than moderate and non-MDMA users on several cognitive parameters but verbal memory performance. They concluded that this was an indication that MDMA use itself was responsible for the cognitive deficits observed in the heavy MDMA users group (Halpern et al., 2004). In theory, the 
approach of this study seemed appealing and looked well-fitted to disclose the effects of MDMA use on cognitive functions. In practice however it turned out that there were statistical and methodological problems. A methodological limitation was that alcohol and cannabis use were not equally distributed over the MDMA group and the control group i.e. the MDMA users used more of these substances. This between-groups difference could have confounded the results, and misrepresented ecstasy use as the cause of deficits in the heavy MDMA user group. Furthermore, Lyvers and Hasking (2004) pointed out some statistical weaknesses e.g. small sample sizes, and lack of correction for alpha inflation. They argue that when appropriate statistics and larger samples were used, it would have been impossible to draw the same conclusion i.e. that MDMA use was responsible for the cognitive impairments in the heavy users group (Lyvers \& Hasking, 2004).

Almost ten years ago, two quasi-experimental studies assessing the acute and residual effects of ecstasy on cognition were conducted. It was shown that when subjects were 'on ecstasy', verbal memory was impaired (Curran \& Travill, 1997; Parrott \& Lasky, 1998). Characteristic of those studies was that they were 'uncontrolled' and conducted according to a 'between groups design'. People who self-administered ecstasy at a party were tested on a range of cognitive measures e.g. verbal memory performance. Their performance was compared with that of a control group that used alcohol, but not ecstasy. Because of the naturalistic nature of the study, certain factors could not be controlled i.e. ecstasy dose and additional use of other substances and this could have confounded the results. It was reported that all subjects used additional substances like alcohol (Curran \& Travill, 1997), amphetamines, cannabis and cocaine (Parrott \& Lasky, 1998) and this polydrug use was not equivalently distributed over the different groups. The quantification of poly-drug use was based on self-reports of the subjects, so this estimation of quantities of substances used could have been unreliable. Furthermore, because subjects were tested at night, the presence of sleep shortage in addition to the ingested drugs could have influenced cognitive functioning. Moreover, in the study of Parrott and Lasky (1998) not all subjects were tested at MDMA's supposed peak concentration. The majority of subjects $(73 \%)$ were tested between 2 to 8 hours after drug intake, 26\% was tested between 8 and 16 hours post-drug. The peak plasma concentration of MDMA rises round 2 hours post drug and the half-life is estimated around 7 or 8 hours post-ingestion (de la Torre et al., 2004). It is possible that the acute effects were already subsiding and that the first 5-HT depletion phase had already set in. Lastly, the comparisons were between subjects' and it is possible that groups were fundamentally different. 
Parrott and Lasky (1998) showed that at baseline, when all groups were drug free, the ecstasy group performed worse than a control group.

Overall, the use of retrospective between-group (but usually far too small groups) comparisons in acute, uncontrolled studies and in studies looking at the long term effects, in addition to the possible confounds (i.e. unknown MDMA dose, polydrug use) have hampered the possibility to ascertain a scientific valid relation between ecstasy use and memory deficits in current and/or abstinent MDMA users. Acute studies with a placebo controlled within subject design however provide a much better tool to investigate the link between MDMA and memory performance. Characteristic for this type of studies is that differences between conditions are directly attributable to the experimental intervention (i.e. drug with a known dosage) and not to randomly occurring between group differences or other confounding factors (e.g. poly-drug use). This experimental placebo-controlled, within subject design was used in the studies presented in this dissertation.

\section{Ecstasy, impulsivity and psychomotor performance}

Ecstasy use has also regularly been associated with impaired decision making and elevated levels of impulsivity (on behavioral measures) in abstinent and current users (Kalant, 2001; Moeller et al., 2002; Morgan, 1998; Morgan et al., 2006). The level of impulsivity has been shown to relate in a negative way to the levels of 5HT in humans (Moeller, Barratt, Dougherty, Schmitz, \& Swann, 2001). This means that when 5-HT levels are high, the degree of impulsivity will be low and vice versa. As it is known that an acute dose of MDMA causes an increased availability of serotonin, it can be suggested that people 'on ecstasy' display a higher degree of impulse control. Otherwise, more impulsive behavior could occur in one of the 5HT depletion phases, i.e. in the first 3 to 6 hours post drug or between 24 hours and 7 days post-drug (Schmidt \& Kehne, 1990). Changes in impulse control are also relevant to daily life activities such as driving. Case studies give an indication that this concern is justified as they have shown that people under the influence of ecstasy display bizarre behavior as demonstrated by speeding and ignoring red traffic lights (Schifano, 1995). Furthermore, epidemiological studies have revealed the involvement of illicit drugs in combination with alcohol in a small percentage (4\%) of the fatalities (Carmen del Rio, Gomez, Sancho, \& Alvarez, 2002). It was also demonstrated that even 'normal' doses of MDMA can cause driver impairment as the MDMA blood plasma levels of a number of impaired drivers 
was comparable with the plasma levels of recreational users (Logan \& Couper, 2003). In a quasi-experimental study by Brookhuis and colleagues (2004) signs of driver impairment were shown after ingestion of multiple drugs. They investigated the effects of MDMA and poly-drug use on driving behavior in a driving simulator, in people who had self-administered ecstasy (average dose 56mg), before going to a party. A major finding was that not so much lateral and longitudinal control was impaired, but that risk-taking behavior increased after MDMA and MDMA in combination with other substances, and sleep loss. This was indicated by the acceptance of smaller gaps between cars and an increase in collisions (Brookhuis, De Waard, \& Samyn, 2004). There were however a number of methodological problems (e.g. uncontrolled poly-drug use, absence of an appropriate control for the nocturnal ride) which limits inferences about the direct effect of ecstasy alone on driving behavior.

In sum, quasi-experimental studies, and studies in abstinent or current users are loaded with methodological problems related with the use of inadequately controlled between-group comparisons in this type of studies, and confounders such as poly-drug use. This complicates the drawing of conclusions on the causality of the association of current ecstasy use with certain adverse behavior e.g. disturbed impulse control, and impaired actual driving performance. Acute studies with a placebo-controlled, within subject design circumvent these methodological problems and therefore provide a much better solution to investigate the relation between MDMA-use and impulsivity, risk-taking behavior and actual driving performance. This means that when differences between conditions are found, it is certain that the intervention and not other confounding factors caused this effect. The studies described in this dissertation are carried out according to this methodologically much more adequate experimental design.

\section{Aim of the dissertation}

The major point from the preceding paragraphs was that although ecstasy use has commonly been associated with cognitive problems in abstinent and/or current users, it has never been possible to ascertain a causal relation. The aim of this dissertation was therefore to investigate the relation between MDMA and these cognitive impairments e.g. impaired memory and elevated impulsivity.

In chapters 2 to 5 the relation between MDMA and memory is the focal point. The acute effects of MDMA on spatial and verbal memory were investigated, and presented in these chapters. In chapters 6,7 , and 8 , the focus lies 
on the relation between MDMA, and impulse control, and actual driving performance. The effects of MDMA alone or in combination with alcohol or sleep deprivation on behavioral measures of impulsivity, and psychomotor performance and on actual driving performance were studied, and presented in these sections.

\section{Methodology}

All the studies described in this dissertation were conducted according to a double blind, placebo controlled within subject design. Some studies also included an active-drug control (methylphenidate) or a reference condition (ethanol). Participants were healthy male and female recreational users of ecstasy. All subjects were healthy as ascertained by a medical examination. Starting point of cognitive tests was always $1.5 \mathrm{~h}$ post drug, at MDMA's peak plasma concentrations.

\section{Outline of the dissertation}

Chapters 2 to 5 describe studies of the acute effects of MDMA on memory performance.

Chapter 2 and chapter 3 describe a study of the acute and withdrawal effects of a single dose of MDMA on verbal and spatial memory performance. Eighteen healthy subjects with previous experience with MDMA use participated in this double-blind placebo-controlled three-way cross-over study. It was hypothesized that verbal and spatial memory performance would benefit from elevated 5-HT levels, and be impaired when 5-HT levels were lowered during the intoxication and the withdrawal phase, respectively. In addition it was assessed whether spatial memory performance under the influence of MDMA was taskdependent. Chapter 4 describes a study of the acute effects of a repeated dose of MDMA during the night and after a night of sleep deprivation, on memory performance. Fourteen healthy subjects with previous experience with ecstasy use participated in this double-blind placebo-controlled two-way cross-over study. It was assessed whether evening doses of MDMA added to or interacted with the memory impairment due to sleep deprivation. Chapter 5 describes the effects of a single dose of MDMA on memory performance and memory related neural structures. Seven healthy subjects with previous experience with ecstasy use participated in this double-blind placebo-controlled two-way cross-over pharmacofMRI study. It was hypothesized that frontal and temporal areas, often linked with 
memory encoding would be especially active during the encoding or word-learning phase and second that MDMA would affect these encoding related areas.

Chapters 6,7, and 8 describe studies of the acute effects of MDMA on impulsivity, psychomotor performance, and actual driving performance.

Chapter 6 describes the effects of MDMA, with and without a low dose of alcohol, on measures of impulsivity. Eighteen healthy subjects with previous experience with the use of ecstasy participated in this double-blind placebocontrolled six-way cross-over study. It was hypothesized that subjects would display an increased impulse control due to elevated 5-HT levels. In addition, it was measured what effects the combination of alcohol with MDMA would have on measures of impulsivity. Chapter 7 describes the effects of nocturnal doses of MDMA throughout the night on measures of psychomotor performance and impulsivity. Fourteen healthy subjects with previous experience with ecstasy use participated in this double-blind placebo-controlled two-way cross-over study. The effects of nocturnal doses of MDMA during the night and after a night of sleep deprivation, on psychomotor performance and impulsivity were assessed. Chapter 8 describes the effects of MDMA, with and without a low dose of alcohol, on actual driving performance. Eighteen healthy subjects with previous experience with the use of ecstasy participated in this double-blind placebo-controlled six-way cross-over study. It was measured whether MDMA had differential effects on a number of aspects of actual driving performance when MDMA was used alone, or used in combination with alcohol.

In chapter 9, several topics related with MDMA use and MDMA research are being reviewed. Additionally some suggestions for further research are presented. It is concluded that the studies presented in this dissertation are unique (because of their design), provide new information, and they are the stepping stones to further profound research in the area of MDMA use, cognition, and psychomotor behavior. 


\section{References}

Barrett, S. P., Gross, S. R., Garand, I., \& Pihl, R. O. (2005). Patterns of Simultaneous Polysubstance Use in Canadian Rave Attendees.

Baylen, C. A., \& Rosenberg, H. (2006). A review of the acute subjective effects of MDMA/ecstasy. Addiction Abingdon, England, 101(7), 933-947.

Britt, G. C., \& McCance Katz, E. F. (2005). A brief overview of the clinical pharmacology of "club drugs". Substance use and misuse, 40(9-10), 1189-1201.

Brookhuis, K. A., De Waard, D., \& Samyn, N. (2004). Effects of MDMA (ecstasy), and multiple drugs use on (simulated) driving performance and traffic safety. Psychopharmacology, 173(3-4), 440-445.

Butler, G. K., \& Montgomery, A. M. (2004). Impulsivity, risk taking and recreational 'ecstasy' (MDMA) use. Drug and alcohol dependence, 76(1), 55-62.

Carmen del Rio, M., Gomez, J., Sancho, M., \& Alvarez, F. J. (2002). Alcohol, illicit drugs and medicinal drugs in fatally injured drivers in Spain between 1991 and 2000. Forensic science international, 127(1-2), 63-70.

Curran, H. V., \& Travill, R. A. (1997). Mood and cognitive effects of +/-3,4methylenedioxymethamphetamine (MDMA, 'ecstasy'): week-end 'high' followed by mid-week low. Addiction Abingdon, England, 92(7), 821-831.

De La Torre, R., \& Farre, M. (2004). Neurotoxicity of MDMA (ecstasy): the limitations of scaling from animals to humans. Trends in pharmacological sciences, 25(10), 505-508.

de la Torre, R., Farre, M., Roset, P. N., Pizarro, N., Abanades, S., Segura, M., et al. (2004). Human pharmacology of MDMA: pharmacokinetics, metabolism, and disposition. Therapeutic drug monitoring, 26(2), 137-144.

DIMS. (2005). DIMS Jaarbericht 2005: Trimbos institute Netherlands Institute of Mental health and Addiction.

EWDD. (2005). Jaarverslag 2005. Stand van de drugsproblematiek in Europa. Luxemburg: Bureau voor officiele publicaties der Europese Gemeenschappen.

Gouzoulis Mayfrank, E., \& Daumann, J. (2006). The confounding problem of polydrug use in recreational ecstasy/MDMA users: A brief overview.

Green, A. R., Mechan, A. O., Elliott, J. M., O'Shea, E., \& Colado, M. I. (2003). The pharmacology and clinical pharmacology of 3,4methylenedioxymethamphetamine (MDMA, "ecstasy"). Pharmacological reviews, 55(3), 463-508.

Hall, A. P., \& Henry, J. A. (2006). Acute toxic effects of 'Ecstasy' (MDMA) and related compounds: overview of pathophysiology and clinical management. British journal of anaesthesia, 96(6), 678-685. 
Halpern, J. H., Pope, H. G., Jr., Sherwood, A. R., Barry, S., Hudson, J. I., \& Yurgelun Todd, D. (2004). Residual neuropsychological effects of illicit 3,4methylenedioxymethamphetamine (MDMA) in individuals with minimal exposure to other drugs. Drug and alcohol dependence, 75(2), 135-147.

Kalant, H. (2001). The pharmacology and toxicology of "ecstasy" (MDMA) and related drugs. CMAJ Canadian Medical Association journal; Journal de l'Association medicale canadienne, 165(7), 917-928.

Logan, B. K., \& Couper, F. J. (2001). 3,4-Methylenedioxymethamphetamine (MDMA, ecstasy) and driving impairment. Journal of forensic sciences, 46(6), 1426-1433.

Logan, B. K., \& Couper, F. J. (2003). 3.4-Methylenedioxymethamphetamine- effects on human performance and behavior. Forensic Sci Rev, 15(11), 12-28.

Lyvers, M., \& Hasking, P. (2004). Have Halpern et al. (2004) detected 'residual neuropsychological effects' of MDMA? Not likely. Drug and alcohol dependence, 75(2), 149-152.

Meeter, M., Talamini, L., Schmitt, J. A., \& Riedel, W. J. (2006). Effects of 5-HT on memory and the hippocampus: model and data. Neuropsychopharmacology official publication of the American College of Neuropsychopharmacology, 31(4), 712-720.

Milroy, C. M. (1999). Ten years of 'ecstasy'. Journal of the Royal Society of Medicine, 92(2), 6871.

Moeller, F. G., Barratt, E. S., Dougherty, D. M., Schmitz, J. M., \& Swann, A. C. (2001). Psychiatric aspects of impulsivity. American journal of psychiatry, 158(11), 1783-1793.

Moeller, F. G., Dougherty, D. M., Steinberg, J. L., Swann, A. C., Silverman, P. B., Ruiz, P., et al. (2002). Heavy "ecstasy" use is associated with increased impulsivity. Addictive Disorders and Their Treatment, 1(2), 47-52.

Morgan, M. J. (1998). Recreational use of "ecstasy" (MDMA) is associated with elevated impulsivity. Neuropsychopharmacology official publication of the American College of Neuropsychopharmacology, 19(4), 252-264.

Morgan, M. J., Impallomeni, L. C., Pirona, A., \& Rogers, R. D. (2006). Elevated impulsivity and impaired decision-making in abstinent Ecstasy (MDMA) users compared to polydrug and drug-naive controls. Neuropsychopharmacology official publication of the American College of Neuropsychopharmacology, 31(7), 1562-1573.

Parrott, A. C. (2001). Human psychopharmacology of Ecstasy (MDMA): A review of 15 years of empirical research. Human Psychopharmacology Clinical and Experimental, 16(8), 557-577.

Parrott, A. C., \& Lasky, J. (1998). Ecstasy (MDMA) effects upon mood and cognition: before, during and after a Saturday night dance. Psychopharmacology, 139(3), 261268. 
Parrott, A. C., Milani, R. M., Parmar, R., \& Turner, J. J. D. (2001). Recreational ecstasy/MDMA and other drug users from the UK and Italy: psychiatric symptoms and psychobiological problems. Psychopharmacology, 159(1), 77-82.

Rattray, M. (1991). Ecstasy: towards an understanding of the biochemical basis of the actions of MDMA. Essays biochem, 26, 77-87.

Reneman, L., de Win, M. M., van den Brink, W., Booij, J., \& den Heeten, G. J. (2006). Neuroimaging findings with MDMA/ecstasy: technical aspects, conceptual issues and future prospects. Journal of psychopharmacology Oxford, England, 20(2), 164-175.

Ricaurte, G. A., Yuan, J., \& McCann, U. D. (2000). (+/-)3,4-

Methylenedioxymethamphetamine ('Ecstasy')-induced serotonin neurotoxicity: studies in animals. Neuropsychobiology, 42(1), 5-10.

Schifano, F. (1995). Dangerous driving and MDMA ("Ecstasy") abuse. Journal of Serotonin Research, 1, 53-57.

Schmidt, C. J., \& Kehne, J. H. (1990). Neurotoxicity of MDMA: neurochemical effects. Annals of the New York Academy of Sciences, 600, 665-680; discussion 680-661.

Soar, K., Turner, J. J. D., \& Parrott, A. C. (2001). Psychiatric disorders in Ecstasy (MDMA) users: A literature review focusing on personal predisposition and drug history. Vol 16(8), 641-645 URLJ: http://www.interscience.wiley.com/ipages/0885$\underline{6222 / .}$

ter Bogt, T. F. M., \& Engels, R. C. M. E. (2005). "Partying" hard: party style, motives for and effects of MDMA use at rave parties. Substance use and misuse, 40(9-10), 14791502.

Thomasius, R., Zapletalova, P., Petersen, K., Buchert, R., Andresen, B., Wartberg, L., et al. (2006). Mood, cognition and serotonin transporter availability in current and former ecstasy (MDMA) users: The longitudinal perspective.

Topp, L., Hando, J., Dillon, P., Roche, A., \& Solowij, N. (1999). Ecstasy use in Australia: patterns of use and associated harm. Drug and alcohol dependence, 55(1-2), 105-115.

Winstock, A. R., Griffiths, P., \& Stewart, D. (2001). Drugs and the dance music scene: a survey of current drug use patterns among a sample of dance music enthusiasts in the UK. Drug and alcohol dependence, 64(1), 9-17. 
22 | CHAPTER 1 


\section{Chapter 2}

\section{TRANSIENT MEMORY IMPAIRMENT AFTER ACUTE DOSE OF $75 \mathrm{MG} \pm 3,4$ METHYLENEDIOXYMETHAMPHETAMINE (MDMA, 'ECSTASY')}

Kuypers, K.P.C. and Ramaekers, J.G.

Background. A range of studies has indicated that users of MDMA display cognitive deficits, particularly memory impairment, as compared to non-drug using controls. Yet it is difficult to determine whether these deficits are caused by MDMA or some other confounding factor, such as poly-drug use. Objective. The present study was designed to establish the direct relation between MDMA and memory impairment under placebo-controlled conditions. Methods. Eighteen recreational MDMA users participated in a double blind, placebo controlled, 3-way crossover design. They were treated with placebo, MDMA $75 \mathrm{mg}$ and methylphenidate $20 \mathrm{mg}$. Memory tests were conducted between 1.5-2 h (intoxication phase) and between 25.5-26 h (withdrawal phase) post dosing. Results. Results showed that a single dose of MDMA caused impairment of immediate and delayed recall on a verbal learning task during the intoxication phase. However, there was no residual memory impairment during the withdrawal phase. Subjects reported more fatigue and less vigor, but no symptoms of depression during the withdrawal phase of MDMA treatment. Methylphenidate did not affect memory or mood at any time of testing. Conclusion. A single dose of MDMA produces transient memory impairment.

Journal of psychopharmacology Oxford, England, 2005; 19(6), 633-639 
24 | CHAPTER 2 


\section{Introduction}

MDMA, the main psychoactive constituent in the popular recreational party drug Ecstasy, has been frequently associated with cognitive impairments (Cole \& Sumnall, 2003; Parrott, 2001). Bolla and colleagues (1998) for example showed impairment of (immediate) verbal memory performance in abstinent MDMA users compared to polydrug non-MDMA users. The magnitude of memory impairment was dose-related and inversely related to the amount of 5HIAA cerebrospinal fluid. They concluded that memory impairment seen in abstinent users could be due to MDMA's presumed neurotoxicity (Bolla et al., 1998). Whether MDMA is neurotoxic in humans however is difficult to infer from these studies for two reasons. First, other markers, like decreased transporter density may have more potential to serve as marker for axonal or structural degeneration (Vollenweider, Gamma, Liechti, \& Huber, 1999). Second, confounding factors may limit interpretation of results from between group designs. A few examples are lifestyle factors that may differ between groups, the dose used that is unknown, and the fact that MDMA users are usually polydrug users so damage can be due to other drugs or combinations (Turner \& Parrott, 2000).

To date, only two studies have made an attempt to assess the effects of MDMA on memory during intoxication (Curran \& Travill, 1997; Parrott \& Lasky, 1998). Both studies employed quasi-experimental, between-subjects designs with multiple test sessions. They assessed mood and cognitive function of regular MDMA users after they self-administered MDMA before going to a party. In the first study (Curran \& Travill, 1997), MDMA users tended to perform worse on memory tasks during intoxication as compared to normal controls, but this difference did not reach statistical significance. The MDMA group did however show significant impairment of long-term memory, the following day. However, the researchers argued that this effect might not be drug related, as the subjects in the MDMA group had suffered from lack of sleep during the night following MDMA use. In the second study (Parrott \& Lasky, 1998) ecstasy users showed marked deficits in memory function after self-administration of MDMA, when compared to normal controls. Yet, results from these studies may suffer from similar interpretational problems as observed in abstinent users. Curran and Travill (1997) for example noted that the use of alcohol and drugs other than MDMA in both control and experimental groups could not be controlled for.

The present study was designed to overcome these interpretational problems in quasi-experimental designs. The study is unique in that it is the first to 
look at the acute and residual effects of a single dose of MDMA (75 mg) on memory functioning in MDMA users in a double-blind, placebo-controlled, crossover design. It was hypothesized that memory function would change as a function of serotonin (5-HT) availability during intoxication and withdrawal. Methylphenidate (Ritalin), a dopaminergic agent, was used as an active control to see whether the effects of MDMA were mediated by dopamine and/ or serotonin.

\section{Methods}

\section{Design and treatments}

The study design was, double-blind, placebo-controlled, 3-way crossover with balancing of the treatments. Testing took place on two consecutive days, on three separate testing periods, with a minimum washout period of 14 days in between. On the first day of each period, participants received either $75 \mathrm{mg}$ of MDMA, 20 $\mathrm{mg}$ of methylphenidate or placebo. MDMA powder was dissolved in $25 \mathrm{ml}$ bitter orange peel syrup and mixed with $200 \mathrm{ml}$ orange juice; and methylphenidate was administered as a pill. All treatments were identically in appearance. Participants received on each first day a solution and a pill in order to blind them, and the researcher, for the treatment. The second day was identical to the first day with the exception that no drug or placebo was given.

\section{Participants}

Eighteen healthy subjects, 9 male and 9 female, aged in the range of 20 to 39 years (mean (SD) 26.22 (5.1)) participated in the study. They all had used MDMA prior to onset of the study. Lifetime use of MDMA varied from light (<30 times) in 15 subjects to heavy (between 60-120 times) in three subjects. They were recruited by means of advertisements in local newspapers and the snowball technique. Potential candidates first underwent a telephonic screening. They were questioned about their drug use and medical condition and were given information about the study. They were sent a detailed brochure with information about the study procedure and two questionnaires for medical history and detailed history of drug use. Inclusion criteria were experience with MDMA use, free from psychotropic medication, good physical health, absence of major medical endocrine and neurological conditions, normal weight (BMI 18-28 kg/m²). Exclusion criteria were history of drug abuse (other than MDMA) or addiction, pregnancy or lactation, cardiovascular abnormalities (assessed by a standard 12-lead electrocardiogram (ECG)), excessive drinking ( $>20$ alcoholic consumptions), 
hypertension (diastolic $>100 \mathrm{mmHg}$; systolic $>170 \mathrm{mmHg}$ ), history of psychiatric or neurological disorders. A medical doctor checked the completed list, and upon approval, they were invited for a medical examination. Blood and urine samples were taken for examination and they underwent an ECG measurement. When they were found medically sound, they were contacted for participation. They were given a brochure with information about the study and a number of rules they had to obey during the period of the study and signed an informed consent to prove they read the information and agreed on it. They were paid upon completion of the testing periods for their participation. The study was performed in accordance with the 1975 declaration of Helsinki, adjusted in Edinburgh (2000) and was approved by the Medical Ethics Committee of the Academic Hospital of Maastricht and the University of Maastricht. A permit for obtaining, storing and administering MDMA was obtained from the Dutch drug enforcement administration.

\section{Study procedure}

Prior to testing days, participants were familiarized with the tests on a training day. Participants were requested to abstain from any drug use one week prior to the medical examination until 14 days after the last testing day. They were asked not to use any caffeinated or alcoholic beverages 24 hours prior to testing and to get a normal night's sleep.

Participants were screened for alcohol use in breath and for recent drug use in urine (THC/ opiates/ cocaine/ amphetamines/ methamphetamines) upon arrival at the testing facilities at $9 \mathrm{am}$. Women were given a pregnancy test. When the results on all of the tests were negative, subjects proceeded with a light breakfast and were given a sleep questionnaire to assess sleep complaints. One hour later subjects received drugs or placebo. One hour and a half after drug intake a blood sample was taken to determine the MDMA/MDA or ritalinic acid concentrations in blood plasma. Following blood sampling, subjects proceeded with the memory tests during the intoxication phase. Similar study procedures were followed when the subjects returned to the laboratory the following day to assess memory performance during withdrawal, i.e. between $25.5-26 \mathrm{~h}$ post dosing. An additional blood sample was taken every 11 th day after drug administration to control for renal- and liver functioning.

\section{Cognitive measures}

Verbal word-learning task. In the verbal word-learning task (Rey, 1958) participants were shown subsequently fifteen monosyllabic words in the center of a computer 
screen; each word was shown for 2 seconds. Their task was to repeat every word aloud upon appearance and recall as many words as possible at the end of the trial. This procedure was repeated five times. The total number of correctly recalled words over 5 trials yielded the immediate recall score (IR), a measure of learning or working memory. After a 30-minute delay, the subject was again asked to recall as many words as possible form the list shown previously. The number of correct recalled words represented the delayed recall score (DR). The DR score was also transformed into a percentage of the highest IR score in the learning trials ((IRDR)/IR) to yield relative recall scores, a measure of forgetting or memory decay. Finally, after the delayed recall, the subject was shown a series of 30 words on the computer display, comprising of the original set and 15 new words in random order. Their task was to indicate which words were parts of the original list. The number of correct recognitions and the average speed (ms) of correct recognitions were recorded as the recognition score (a measure of long term memory) and the recognition time (RT, a measure of speed of retrieval from long term memory). Parallel versions of the verbal word-learning task were used in each test replication to prevent learning effects over time.

Syntactic reasoning task. In the syntactic reasoning task (Baddeley, 1968) participants were shown subsequently 32 sentences in the center of a computer screen describing the order of two letters, each followed by a letterpair ( $\mathrm{BB}$ or BA). Half of the letter pairs showed the same order as in the preceding sentence, and half of the pairs the opposite. Sentence difficulty varied within the series, from simple active sentences to more complicated sentences involving passives, negatives or both; e.g. "B is not followed by A". Participants had to decide, as quickly as possible, by means of pressing a yes or no button whether the letter pair matched the descriptive sentence. The number of correctly categorized items can be considered as a measure of working memory and reaction time of correct answers as a measure of speed of working memory.

Digit Symbol Substitution Task. The digit symbol substitution task used here was a computerized version of the paper and pencil task from the WAIS (Wechsler, 1981; Ramaekers et al., 1999). It assesses short-term memory and psychomotor performance. Participants were shown an encoding scheme which linked numbers of 1 to 9 to nine different symbols. The same row of symbols was shown at the bottom of the screen as response buttons. In the center of the screen, digits appeared. The task of the subject was to choose the correct symbol that was linked 
to the digit by means of moving the cursor to the correct symbol in the bottom row and clicking on it. The number of digits correctly encoded within 3 minutes was the performance measure.

\section{Subjective measures}

Three subjective measurements were taken: i.e. the Groninger Sleep Scale (GSS), the Profile of Mood States (POMS) and the Hamilton Depression Rating Scale (HAM-D).

The GSS assesses sleep quality and quantity (hours of sleep). It consists of fifteen dichotomous questions about sleep complaints and an open question concerning the duration of sleep. The POMS is a self-assessment mood questionnaire with 32 visual analogue scales representing five mood states; that is Depression, Vigor, Tension, Fatigue and Anger. On both ends of each scale there are words reflecting opposite moods. The participant had to indicate in how far these items were representing his or her mood.

\section{Pharmacokinetics}

Blood samples were collected in $5 \mathrm{ml}$ glass tubes containing sodium fluoride and potassium oxalate. After centrifugation at $4000 \mathrm{~g}$ for $10 \mathrm{~min}$, the resultant plasma was transferred to a clean tube and frozen at $-20^{\circ} \mathrm{C}$ until LC-MS/MS analysis. The limit of quantification for MDMA, MDA, and ritalinic acid was $1 \mathrm{ng} / \mathrm{ml}$.

\section{Statistical Analyses}

All statistical analyses were conducted by means of SPSS 11.5 for Windows. Each objective parameter was analysed using GLM univariate repeated measures procedures with Treatment ( 3 levels) and Day of testing (2 levels) as main within subject factors. In case of the immediate recall scores, Trial (5 levels) was added as an additional factor. Separate contrast (drug vs placebo) tests were conducted in case of a significant overall effect of Treatment or Treatment by Day.

\section{Results}

\section{Missing data}

In the Placebo condition, one subject completed only 50\% of the POMS questionnaire on two consecutive days. Missing values were replaced by the mean of scores of the other 17 subjects in the same condition. 


\section{Cognitive measures}

Verbal word-learning task. ANOVA revealed significant main effects of Treatment by Day $\left(\mathrm{F}_{2,34}=7.58 ; \mathrm{p}=0.002\right)$ and Trial $\left(\mathrm{F}_{4,68}=214.86 ; \mathrm{p}<0.001\right)$ on immediate recall scores. The latter effect reflects the overall increase in the number of words recalled over 5 subsequent learning trials (see Fig 1). The former effect was further analysed by means of separate drug placebo contrasts on each Day of testing. These revealed a significant difference between MDMA and placebo $(p=0.009)$ on day 1. During MDMA intoxication subjects learned less words as compared to placebo (See Fig. 1). The mean (SE) difference from placebo summed over 5 trials was 4.6 (1.7) words. There were no differences between drugs and placebo on day 2 of testing during withdrawal.

Delayed recall scores revealed significant main effects of Day $\left(\mathrm{F}_{1,17}=5.36\right.$; $\mathrm{p}=0.033)$ and Treatment by Day $\left(\mathrm{F}_{2,34}=4.72 ; \mathrm{p}=0.016\right)$. Subjects generally remembered less during delayed recall on day 2 (mean (SE) $0.83(0.36)$ ) as compared to day 1 . Separate drug-placebo contrasts revealed a significant difference between MDMA and placebo $\left(\mathrm{F}_{1,17}=9.91 ; \mathrm{p}=0.006\right)$ on day 1 but no differences between drugs and placebo on day 2. While under influence of MDMA participants remembered approximately 1.44 (SE 0.46) words less during delayed recall, compared to placebo.

Relative recall scores analysis showed main effects of Day $\left(\mathrm{F}_{1,17}=7.30 ; \mathrm{p}=\right.$ $0.015)$ and Treatment by Day $\left(\mathrm{F}_{2,34}=3.43 ; \mathrm{p}=0.044\right)$. Treatment contrasts revealed a nearly significant difference between MDMA and placebo $\left(\mathrm{F}_{1,17}=3.5 ; \mathrm{p}=0.079\right)$ on day 1 but no differences between drugs and placebo on day 2. Recognition scores were not affected by Treatment, Day or their interaction (Table 1).

Syntactic reasoning task. Except for a main effect of Day $\left(\mathrm{F}_{1,17}=9.63 ; \mathrm{p}=0.006\right)$ on reaction time of correctly categorized items, there were no significant main effects of Treatment, Day or Treatment by Day on the variables of the syntactic reasoning task (See Table 1). Subjects appeared to react faster on day 2 (92.01; SE 29.64) compared to day 1.

Digit-Symbol Substitution Task. Apart from a significant main effect of Day $\left(\mathrm{F}_{1,17}=\right.$ 25.87; $\mathrm{p}=0.001)$, there were no significant effects of Treatment or Treatment by Day on number of correctly encoded digits.Subjects encoded on average more digits correctly on day 2 (3.96, SE 0.78) compared to day 1 (Table 1). 


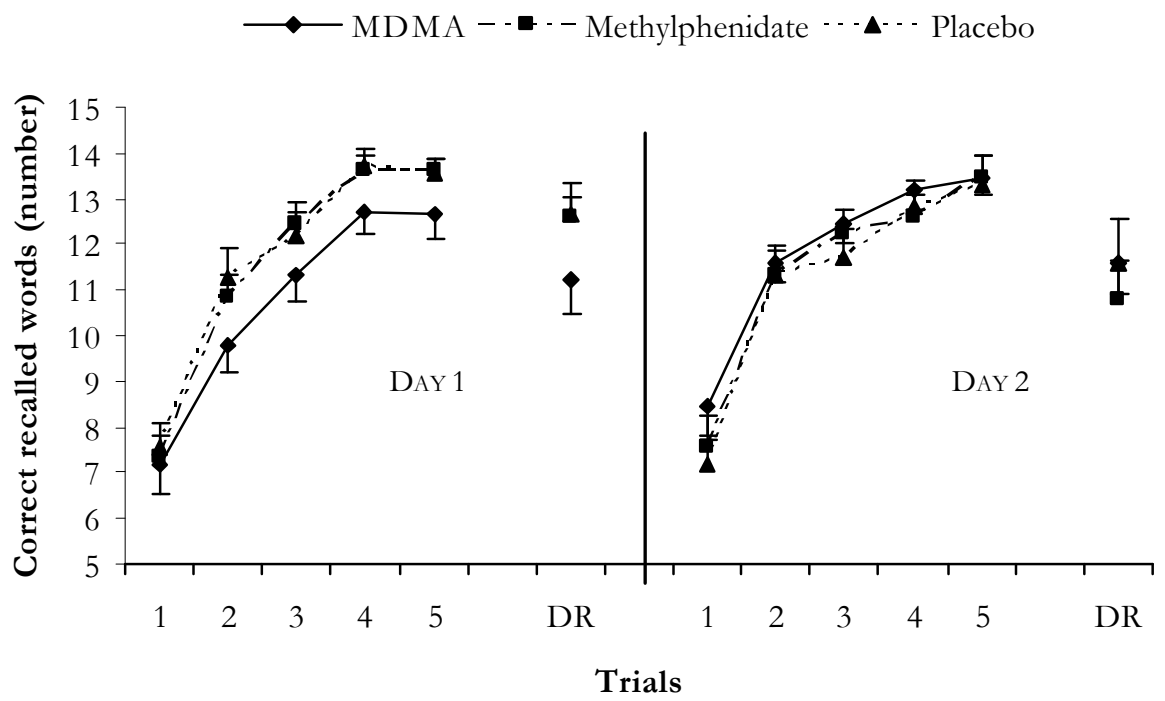

Figure 1. In this figure the number of correctly recalled words on the fifteen word-learning task on the five immediate recall trials and the delayed recall (DR) trial on day 1 (acute phase) and day 2 (withdrawal phase) is shown.

\section{Subjective Measures}

There were no significant main effects of Treatment, Day or Treatment by Day on Sleep Quantity or Quality scores. Subjects slept on average 7.28h (SE 0.19) and had an average score of 2.28 (SE 0.39) on the sleep complaints questionnaire. There were no significant main effects of Treatment or Day on the five subscales of the POMS. There were however significant interaction effects of Treatment by Day on two subscales measuring fatigue $\left(\mathrm{F}_{2,34}=3.56 ; \mathrm{p}=0.039\right)$ and vigor $\left(\mathrm{F}_{2,34}=\right.$ 6.15; $\mathrm{p}=$ 0.005). Drug-placebo contrasts on day 1 revealed no significant differences between MDMA, methylphenidate and placebo. On day 2 however MDMA increased subjective feelings of fatigue $\left(\mathrm{F}_{1,17}=6.06 ; \mathrm{p}=0.025\right)$ and decreased vigor $\left(\mathrm{F}_{1,17}=8.71 ; \mathrm{p}=0.009\right)$ relative to placebo. There were no significant effects of Treatment or Treatment by Day on the total score of the Hamilton-D. Analysis of Hamilton-D scores did reveal a significant main effect of Day $\left(\mathrm{F}_{1,17}=5.02 ; \mathrm{p}=0.039\right)$ indicating that scores were higher on day 1 as compared to day 2 (Table 2).

\section{Pharmacokinetics}

Plasma concentrations of MDMA, MDA and ritalinic acid were determined 1.5 hours (day 1 ) and 25.5 hours (day 2) post-drug. Concentrations of MDMA were on average $113.4 \mathrm{ng} / \mathrm{ml}( \pm 37.4)$ and $11.6 \mathrm{ng} / \mathrm{ml}( \pm 9.3)$ respectively on day 1 and 2 . 
Concentrations of MDA were on average $2.9 \mathrm{ng} / \mathrm{ml}( \pm 0.9)$ and $2.1 \mathrm{ng} / \mathrm{ml}( \pm 1.2)$ respectively on day 1 and 2 . Concentrations of ritalinic acid were on average 95.9 $\mathrm{ng} / \mathrm{ml}( \pm 78.4)$ and $22.9 \mathrm{ng} / \mathrm{ml}( \pm 7)$ respectively on day 1 and 2 .

\section{Discussion}

A single dose of MDMA impaired memory during the intoxication phase. This impairment was no longer visible the day after, during the withdrawal phase. In the intoxication phase, working memory, as assessed by the number of words recalled in the immediate recall trials of the word-learning task, was impaired. Subjects showed a normal learning curve over the five trials, that is, every trial they could recall more words compared to the previous trial, but in total, they learned fewer words following MDMA as compared to placebo. In addition, the delayed recall trial was also affected after MDMA. Subjects recalled fewer words after a delay of 30 minutes compared to placebo. An almost significant reduction of the relative recall score furthermore demonstrated that this effect was not only attributable to a lesser amount of words learned in the immediate recall trials but also to a faster forgetting rate. This pattern of impairment of immediate and delayed recall is similar to the pattern of memory deficits shown in abstinent users of MDMA (Parrott, 2001). The similarity in memory impairment observed in abstinent MDMA users and that during acute MDMA intoxication strengthen the notion that these phenomena may share a common source or neuro-pharmacological brain structure.

Verbal memory was not impaired in the withdrawal phase of MDMA. Both immediate and delayed recall scores showed no deviations from performance in the placebo condition. Subjects learned a comparable number of words and produced the same learning curve as in the placebo condition. They also recalled a comparable number of words after a 30-minute delay and had normal forgetting rates as shown by the relative recall score that was the same in the placebo condition. These findings show that a single dose of MDMA does not cause permanent memory damage.

It has been noted before that memory impairment during withdrawal may be secondary to the development of depressive symptoms during the mid-week. Curran and Travill (1997), and Parrott and Lasky (1998) were the first to describe symptoms of depression in subjects between 2 to 5 days following the use of MDMA. In the present study, mood assessments were also included in the study protocol. On day 2, during withdrawal, subjects appeared to be less vigorous and 
more fatigued as compared to placebo. However, there were no signs of depression following MDMA treatment, as measured by the HAM-D or the Profile of Mood State. The failure of the present study to replicate sub-acute mood effects reported by Curran and Travill (1997) and Parrot and Lasky (1998) may be related to the fact that the latter conducted their quasi-controlled studies in nightclubs. Many subjects in these nightclub studies were polydrug users who attested to the use of combinations of MDMA with alcohol, cannabis or cocaine (Parrott and Lasky, 1998) or suffered from lack of sleep when tested the day after. It can thus not be excluded that depressive symptoms reported in these nightclub studies may have been confounded by such factors as polydrug use or sleep deprivation.

Methylphenidate did not affect memory performance. This finding indicates that MDMA-induced memory impairment is related to the drugs' serotonergic, but not its dopaminergic mechanism of action. Bray and colleagues (2004) previously also found this absence of effects of methylphenidate on verbal memory. Sleep-deprived subjects, which were administered methylphenidate (20 $\mathrm{mg}$ ), performed equally well compared to a sleep-deprived control group on a verbal memory task. Apparently, methylphenidate does not seem to play a role in verbal memory, at least not at the dose that was used by Bray and colleagues (2004) and the present study. Thus, memory impairment, observed after administration of MDMA is likely to be caused by manipulation of the serotonergic system.

The exact mechanism underlying memory impairment following acute MDMA administration is presently unknown. There are several candidate explanations, one of which is 5-HT depletion. From animal studies, it is known that administration of MDMA causes two serotonin depletion phases. Single doses of MDMA diminish tryptophan hydroxylase, responsible for serotonin synthesis, and increase release of 5-HT at the same time. The net result of these two processes is lowered serotonin concentrations during the first 3 to 6 hours postdrug. This is the first depletion phase, also known as the acute or reversible phase. Hereafter a gradual recovery of serotonin levels takes place, followed by a second depletion phase (neurotoxic or long-term phase) 24 hours, to a week post- drug (Schmidt \& Kehne, 1990). Perhaps these fluctuations in serotonin concentrations can account for the results of the present study. That is, depleted serotonin levels may account for the impairment in memory seen 1.5 hours after MDMA administration. The absence of memory impairment 25.5 hours post-drug may have resulted from a normalization of 5-HT levels following the first depletion phase. Still, it should be noted that tryptophan depletion has generally failed to 
affect working memory as measured in immediate word recall tasks (Riedel, Klaassen, Deutz, van Someren, \& van Praag, 1999; Schmitt et al., 2000). What seems typical however to MDMA induced memory deficits is a reduction in both immediate and delayed word recall. This impairment pattern was shown in the present study during acute MDMA intoxication and in previous studies assessing memory in abstinent MDMA users (Parrott, 2001).

The qualitative differences between memory impairments observed after MDMA intoxication and tryptophan depletion raises the question whether MDMA exerts its detrimental effect on memory through other mechanisms than synaptic 5-HT depletion alone. Alternative mechanisms that may account for memory impairment during MDMA intoxication include indirect agonism of postsynaptic 5-HT $\mathrm{H}_{\mathrm{a}}$ and direct activation of 5- $\mathrm{HT}_{2 \mathrm{a}}$ receptors. Yasuno and colleagues (2003) showed that stimulation of postsynaptic 5-HT $\mathrm{T}_{1 \mathrm{a}}$ receptors in the hippocampus produced dose-related short- and long-term (recognition) memory impairment in humans. In addition, PET studies have shown a negative correlation between memory function and $5-\mathrm{HT}_{1 \mathrm{~A}}$ receptor agonists binding in the hippocampus. It is also known that $5-\mathrm{HT}_{1}$ receptors possess very high binding affinities for 5 -HT (Glennon \& Dukat, 1995). Thus, an increment in 5-HT release following acute MDMA administration may indirectly stimulate postsynaptic $5-\mathrm{HT}_{1 \mathrm{a}}$ receptors.

Reneman and colleagues (2000) have shown that $5 \mathrm{HT}_{2 \mathrm{a}}$ receptor binding was associated with memory impairment in abstinent MDMA users (Reneman, Booij, Schmand, van den Brink, \& Gunning, 2000). It is clear from animal studies that $5-\mathrm{HT}_{2}$ receptors mediate learning and memory processes, although it is not completely clear whether $5-\mathrm{HT}_{2}$ receptor drugs achieve their facilitating and impairing effects through agonism, antagonism or inverse agonism (Meneses, 2002). Still, there appears to be some consensus that $5-\mathrm{HT}_{2 \mathrm{~A} / 2 \mathrm{C}}$ blockade improves learning whereas $5-\mathrm{HT}_{2}$ receptor agonists such as $\mathrm{mCPP}$ have been shown to decrease learning and memory in rats (Meneses, 1999). The same mechanism might be responsible for the decrement in learning and delayed recall in the present experiment, as it has been previously demonstrated that MDMA possesses a moderate affinity for activating the 5-HT2A receptor (Battaglia, Brooks, Kulsakdinun, \& De Souza, 1988).

In conclusion, data from the present study showed that a single dose of MDMA causes memory impairment during intoxication. It was demonstrated that subjects recalled fewer words during immediate and delayed recall of a wordlearning task while under the influence of MDMA. Yet, memory impairment was also transient and no longer present the following day. 
Table 1. Summary of means (SE) and Treatment by Day interaction effects in cognitive tasks. (Overall $D f=2,34)(M P=$ methylphenidate; $P l=$ Placebo $)$

\begin{tabular}{|c|c|c|c|c|c|}
\hline Task & Day & MDMA & $\begin{array}{l}\text { TREATMENT } \\
\text { MP }\end{array}$ & \multicolumn{2}{|c|}{ Placebo } \\
\hline \multicolumn{6}{|c|}{ VERBAL WORD-LEARNING TASK } \\
\hline IR (Trial 1) & \multirow{6}{*}{1} & $7.17(0.61)$ & $7.33(0.46)$ & \multirow{2}{*}{\multicolumn{2}{|c|}{$\begin{array}{c}7.56(0.56) \\
1128(063)\end{array}$}} \\
\hline IR (Trial 2) & & $9.78(0.60)$ & $10.83(0.51)$ & & \\
\hline IR (Trial 3) & & $11.33(0.59)$ & $12.44(0.50)$ & \multirow{2}{*}{\multicolumn{2}{|c|}{$\begin{array}{l}12.17(0.54) \\
13.72(0.36)\end{array}$}} \\
\hline IR (Trial 4) & & $12.72(0.50)$ & 13.61(0.30) & & \\
\hline IR (Trial 5) & & $12.67(0.55)$ & $13.61(0.29)$ & \multicolumn{2}{|c|}{$\begin{array}{l}13.72(0.36) \\
13.55(0.32)\end{array}$} \\
\hline IR (Trial 1) & & $8.44(0.64)$ & $7.55(0.69)$ & \multicolumn{2}{|c|}{$7.17(0.52)$} \\
\hline IR ('Trial 2) & \multirow{4}{*}{2} & $11.61(0.46)$ & $11.33(0.55)$ & \multicolumn{2}{|c|}{$11.33(0.64)$} \\
\hline IR (Trial 3) & & $12.44(0.42)$ & $12.22(0.55)$ & \multicolumn{2}{|c|}{$11.72(0.62)$} \\
\hline IR (Trial 4) & & $13.17(0.41)$ & $12.61(0.47)$ & \multirow{2}{*}{\multicolumn{2}{|c|}{$12.83(0.59)$}} \\
\hline IR (Trial 5) & & $13.44(0.37)$ & $13.44(0.47)$ & 13.28(0.64) & \\
\hline IR (Total) & 1 & $53.67(2.39)$ & $57.83(1.81)$ & \multirow{2}{*}{\multicolumn{2}{|c|}{$\begin{array}{l}58.28(2.06) \\
56.33(2.82)\end{array}$}} \\
\hline & 2 & $59.11(1.93)$ & $57.17(2.41)$ & & \\
\hline DR & 1 & $11.22(0.75)$ & $12.61(0.44)$ & \multicolumn{2}{|c|}{$\begin{array}{l}56.33(2.82) \\
12.67(0.66)\end{array}$} \\
\hline & 2 & $12.61(0.71)$ & $10.78(0.86)$ & \multicolumn{2}{|c|}{$\begin{array}{l}12.67(0.66) \\
11.61(0.97)\end{array}$} \\
\hline $\mathrm{RR}$ & 1 & $0.18(0.04)$ & $0.09(0.03)$ & \multicolumn{2}{|c|}{$0.10(0.03)$} \\
\hline & 2 & $0.17(0.04)$ & $0.23(0.05)$ & \multicolumn{2}{|c|}{$0.17(0.05)$} \\
\hline Recognition & 1 & $28.83(0.47)$ & $29.00(0.31)$ & \multirow{2}{*}{\multicolumn{2}{|c|}{$\begin{array}{l}29.39(0.24) \\
28.61(0.40)\end{array}$}} \\
\hline & 2 & $28.50(0.64)$ & $28.67(0.47)$ & & \\
\hline RT Recognition & 1 & $665.82(18.14)$ & $665.17(16.78)$ & \multicolumn{2}{|c|}{$648.90(15.30)$} \\
\hline & 2 & $689.82(37.08)$ & $666.80(15.35)$ & \multicolumn{2}{|c|}{$652.69(18.63)$} \\
\hline \multicolumn{6}{|c|}{ SYNTACTICAL REASONING TASK } \\
\hline Correct total & 1 & $24.06(1.19)$ & $24.78(1.26)$ & & \\
\hline & 2 & $24.44(1.37)$ & $25.33(1.37)$ & 25.17 & $(1.45)$ \\
\hline RT & 1 & $1458.56(89.89)$ & $1474.45(98.44)$ & 1531.6 & $4(77.99)$ \\
\hline & 2 & 1347.71(97.86) & $1430.47(90.22)$ & 1410.45 & $5(93.85)$ \\
\hline DIGIT-SYMBOL SL & 3STITU & N TASK & & & \\
\hline Encoded digits & 1 & $84.94(3.28)$ & $85.89(3.79)$ & 86.94 & (3.59) \\
\hline (\# correct) & 2 & $88.56(3.76)$ & $89.67(3.21)$ & 91.44 & (3.53) \\
\hline & & & ANOVA & & \\
\hline & & Overall ( $\mathrm{T}$ & ent by day) & & \\
\hline Task & Day & $\mathrm{F}$ & $\mathrm{p}$ & Con & trasts \\
\hline VERBAL WORD LF & RNIN & & & MDMA/PL & $\mathrm{MP} / \mathrm{PL}$ \\
\hline IR (Trial 1-5) & $\begin{array}{l}1 \\
2\end{array}$ & 7.58 & 0.002 & $\begin{array}{c}0.026 \\
-\end{array}$ & - \\
\hline IR (Total) & $\begin{array}{l}1 \\
2\end{array}$ & 7.58 & 0.002 & 0.026 & - \\
\hline DR & 1 & 4.72 & 0.016 & 0.006 & - \\
\hline $\mathrm{RR}$ & 1 & 3.43 & 0.044 & 0.079 & - \\
\hline Recononition & $\begin{array}{l}2 \\
1\end{array}$ & & & $\begin{array}{l}- \\
-\end{array}$ & - \\
\hline Kecognition & $\begin{array}{l}1 \\
2\end{array}$ & - & - & $\begin{array}{c}- \\
-\end{array}$ & - \\
\hline RT Recognition & 1 & & & - & - \\
\hline & 2 & - & - & - & - \\
\hline SYNTACTIC REASC & NING ${ }^{-1}-4$ & & & & \\
\hline Correct (total) & 1 & - & - & - & - \\
\hline RT & $\begin{array}{l}2 \\
1\end{array}$ & - & - & $\begin{array}{l}- \\
-\end{array}$ & $\begin{array}{l}- \\
-\end{array}$ \\
\hline 111 & 2 & - & - & - & - \\
\hline DIGIT-SYMBOL SI & BSTITL & N TASK & & - & \\
\hline $\begin{array}{l}\text { Encoded digits } \\
\text { (\# correct) }\end{array}$ & $\begin{array}{l}1 \\
2\end{array}$ & - & - & - & - \\
\hline
\end{tabular}


36 | CHAPTER 2

Table 2. In this table, a summary of means (SE) and Treatment by Day interaction effects in subjective evaluations are shown. (Overall $D f=2,34)(M P=$ methylphenidate)

\begin{tabular}{|c|c|c|c|c|c|}
\hline & & & TREATMF & \multirow{2}{*}{\multicolumn{2}{|c|}{ Placebo }} \\
\hline Questionnaire & Day & MDMA & MP & & \\
\hline \multicolumn{6}{|c|}{ SLEEP QUESTIONNAIRE } \\
\hline Quality & 1 & $2.22(0.59)$ & $3.56(0.94)$ & \multicolumn{2}{|c|}{$2.67(0.66)$} \\
\hline & 2 & $1.72(0.61)$ & $2.00(0.48)$ & \multicolumn{2}{|c|}{$1.50(0.78)$} \\
\hline Quantity & 1 & $6.94(0.22)$ & $6.76(0.32)$ & \multicolumn{2}{|c|}{ 7.67(0.48) } \\
\hline & 2 & $7.78(0.33)$ & $7.25(0.28)$ & \multicolumn{2}{|c|}{$7.25(0.39)$} \\
\hline \multicolumn{6}{|l|}{ POMS } \\
\hline Depression & 1 & $64.80(1.87)$ & $64.52(2.06)$ & \multicolumn{2}{|c|}{$61.02(3.09)$} \\
\hline & 2 & $61.93(2.89)$ & $62.30(2.72)$ & \multicolumn{2}{|c|}{$64.59(2.70)$} \\
\hline Anger & 1 & $58.31(1.24)$ & $57.69(1.68)$ & \multicolumn{2}{|c|}{$55.09(2.42)$} \\
\hline & 2 & $55.59(2.48)$ & $54.98(2.26)$ & \multicolumn{2}{|c|}{$56.38(1.97)$} \\
\hline Fatigue & 1 & $41.31(2.88)$ & $38.56(2.80)$ & \multicolumn{2}{|c|}{$35.98(3.22)$} \\
\hline & 2 & $37.29(2.98)$ & $38.99(2.59)$ & \multicolumn{2}{|c|}{$42.94(2.36)$} \\
\hline Vigor & 1 & $12.88(2.11)$ & $15.46(2.24)$ & \multicolumn{2}{|c|}{$18.11(2.71)$} \\
\hline & 2 & $18.18(2.32)$ & $16.12(2.25)$ & \multicolumn{2}{|c|}{$12.97(2.00)$} \\
\hline Tension & 1 & 45.01(1.97) & $47.01(1.81)$ & \multicolumn{2}{|c|}{$47.81(1.66)$} \\
\hline & 2 & $47.20(1.83)$ & $47.79(1.74)$ & \multicolumn{2}{|c|}{$48.42(1.74)$} \\
\hline \multicolumn{6}{|l|}{ HAM-D } \\
\hline \multirow[t]{2}{*}{ Score } & 1 & $1.72(0.54)$ & $1.39(0.44)$ & \multirow{2}{*}{\multicolumn{2}{|c|}{$\begin{array}{l}1.72(0.59) \\
1.28(0.38)\end{array}$}} \\
\hline & 2 & $1.33(0.28)$ & $1.00(0.37)$ & & \\
\hline Questionnaire & Day & \multicolumn{2}{|c|}{$\begin{array}{c}\text { ANOV } \\
\text { Overall (Treatment by dav) }\end{array}$} & \multicolumn{2}{|c|}{ Contrasts } \\
\hline \multicolumn{2}{|c|}{ SLEEP QUESTIONNAIRE } & $\mathrm{F}$ & $\mathrm{P}$ & MDMA/PL & $\mathrm{MP} / \mathrm{PL}_{\mathrm{L}}$ \\
\hline Quality & 1 & \multirow[b]{2}{*}{-} & \multirow[t]{2}{*}{ 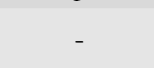 } & \multirow{2}{*}{\multicolumn{2}{|c|}{$\begin{array}{lll}- & - \\
- & -\end{array}$}} \\
\hline & 2 & & & & \\
\hline Quantity & 1 & - & - & - & - \\
\hline & 2 & - & - & - & - \\
\hline POMS & & & & & \\
\hline Depression & 1 & & & - & - \\
\hline & 2 & - & - & - & - \\
\hline Anger & 1 & - & - & - & - \\
\hline & 2 & - & - & - & - \\
\hline Fatigue & 1 & 3.56 & 0.039 & - & - \\
\hline & 2 & 5.50 & 0.039 & 0.025 & - \\
\hline Vigor & 1 & 6.15 & 0.005 & - & - \\
\hline & 2 & 0.15 & 0.005 & 0.009 & - \\
\hline Tension & 1 & & & - & - \\
\hline & 2 & - & - & - & - \\
\hline HAM-D & & & & & \\
\hline Score & 1 & - & - & - & - \\
\hline & 2 & - & - & - & - \\
\hline
\end{tabular}

\section{Acknowledgements}

We would like to thank Nele Samyn, Gert De Boeck and Marleen Laloup from NICC, Brussels, for analyzing MDMA and methylphenidate blood plasma samples.

This study was part of the IMMORTAL project, funded by the European Commission (Grant GMA1-2000-27043). 


\section{References}

Baddeley, A. D. (1968). A 3 Min Reasoning Test Based on Grammatical Transformation. Psychonomic Science, 10(10), 341-342.

Battaglia, G., Brooks, B. P., Kulsakdinun, C., \& De Souza, E. B. (1988). Pharmacologic profile of MDMA (3,4-methylenedioxymethamphetamine) at various brain recognition sites. European journal of pharmacology, 149(1-2), 159-163.

Bolla, K. I., McCann, U. D., \& Ricaurte, G. A. (1998). Memory impairment in abstinent MDMA ("Ecstasy") users. Neurology, 51(6), 1532-1537.

Bray, C. L., Cahill, K. S., Oshier, J. T., Peden, C. S., Theriaque, D. W., Flotte, T. R., et al. (2004). Methylphenidate does not improve cognitive function in healthy sleepdeprived young adults. Journal of investigative medicine the official publication of the American Federation for Clinical Research, 52(3), 192-201.

Cole, J. C., \& Sumnall, H. R. (2003). Altered states: the clinical effects of Ecstasy. Pharmacology and Therapeutics, 98, 35-58.

Curran, H. V., \& Travill, R. A. (1997). Mood and cognitive effects of +/-3,4methylenedioxymethamphetamine (MDMA, 'ecstasy'): week-end 'high' followed by mid-week low. Addiction Abingdon, England, 92(7), 821-831.

Glennon, R. A., \& Dukat, M. (1995). Serotonin receptor subtypes and ligands. In F. E. K. Bloom, D.J. (Ed.), Psychopharmacology: The fourth generation of progress (pp. 415-429). New York: Raven Press.

Meneses, A. (1999). 5-HT system and cognition. Neuroscience and biobehavioral reviews, 23(8), 1111-1125.

Meneses, A. (2002). Involvement of 5-HT(2A/2B/2C) receptors on memory formation: simple agonism, antagonism, or inverse agonism? Cellular and molecular neurobiology, 22(5-6), 675-688.

Parrott, A. C. (2001). Human psychopharmacology of Ecstasy (MDMA): A review of 15 years of empirical research. Human Psychopharmacology Clinical and Experimental, 16(8), 557-577.

Parrott, A. C., \& Lasky, J. (1998). Ecstasy (MDMA) effects upon mood and cognition: before, during and after a Saturday night dance. Psychopharmacology, 139(3), 261268.

Ramaekers, J. G., Louwerens, J. W., Muntjewerff, N. D., Milius, H., de Bie, A., Rosenzweig, P., et al. (1999). Psychomotor, Cognitive, extrapyramidal, and affective functions of healthy volunteers during treatment with an atypical (amisulpride) and a classic (haloperidol) antipsychotic. Journal of clinical psychopharmacology, 19(3), 209-221. 
Reneman, L., Booij, J., Schmand, B., van den Brink, W., \& Gunning, B. (2000). Memory disturbances in "Ecstacy" users are correlated with an altered brain serotonin neurotransmission. Psychopharmacology, 148(3), 322-324.

Rey, A. (1958). The clinical examination in psychology / L'examen clinique en psychologie: Oxford, England: Presses Universitaries De France.

Riedel, W. J., Klaassen, T., Deutz, N. E., van Someren, A., \& van Praag, H. M. (1999). Tryptophan depletion in normal volunteers produces selective impairment in memory consolidation. Psychopharmacology, 141(4), 362-369.

Schmidt, C. J., \& Kehne, J. H. (1990). Neurotoxicity of MDMA: neurochemical effects. Annals of the New York. Academy of Sciences, 600, 665-680; discussion 680-661.

Schmitt, J. A., Jorissen, B. L., Sobczak, S., van Boxtel, M. P., Hogervorst, E., Deutz, N. E., et al. (2000). Tryptophan depletion impairs memory consolidation but improves focussed attention in healthy young volunteers. Journal of psychopharmacology Oxford, England, 14(1), 21-29.

Turner, J. J., \& Parrott, A. C. (2000). 'Is MDMA a human neurotoxin?': diverse views from the discussants. Neuropsychobiology, 42(1), 42-48.

Vollenweider, F. X., Gamma, A., Liechti, M., \& Huber, T. (1999). Is a single dose of MDMA harmless? Neuropsychopharmacology official publication of the American College of Neuropsychopharmacology, 21(4), 598-600.

Wechsler, D. (1981). The psychometric tradition: Developing the Wechsler Adult Intelligence Scale. Contemporary Educational Psychology, 6(2), 82-85.

Yasuno, F., Suhara, T., Nakayama, T., Ichimiya, T., Okubo, Y., Takano, A., et al. (2003). Inhibitory effect of hippocampal 5-HT-sub(1A ) receptors on human explicit memory. American Journal of Psychiatry, 160(2), 334-340. 


\section{CHAPTER 3}

\section{ACUTE DOSE OF MDMA (75MG) IMPAIRS SPATIAL MEMORY FOR LOCATION BUT LEAVES CONTEXTUAL PROCESSING OF VISUOSPATIAL INFORMATION UNAFFECTED}

Kuypers, K.P.C., and Ramaekers, J.G.

Rationale. Research concerning spatial memory in MDMA users has presented conflicting results showing either the presence or absence of spatial memory deficits. Two factors may have confounded results in abstinent users: memory task characteristics and polydrug use. Objectives. The present study aims to assess whether a single dose of MDMA affects spatial memory performance during intoxication and withdrawal phase and whether spatial memory performance after MDMA is task dependent. Methods. Eighteen recreational MDMA users participated in a double-blind, placebo-controlled, 3-way crossover design. They were treated with placebo, MDMA (75 mg), and methylphenidate $(20 \mathrm{mg})$. Memory tests were conducted between 1.5 and $2 \mathrm{~h}$ (intoxication phase) and between 25.5 and $26 \mathrm{~h}$ (withdrawal phase) post-dosing. Two spatial memory tasks of varying complexity were used that required either storage of stimulus location alone (spatial memory task) or memory for location as well as processing of content or contextual information (change blindness task). Results. After a single dose of MDMA subjects made larger localization errors and responded faster compared to placebo in the simple spatial memory task during intoxication phase. Inaccuracy was not due to increased response speed, as determined by regression analysis. Performance in the change blindness task was not affected by MDMA. Methylphenidate did not affect performance on any of the tasks. Conclusion. It is concluded that a single dose of MDMA impairs spatial memory for location but leaves processing of contextual information intact.

Psychopharmacology, 2007; 198 (4), 557-563 
40 | CHAPTER 3 


\section{Introduction}

3,4-Methylenedioxymethamphetamine (MDMA, Ecstasy) has been frequently associated with cognitive impairments in abstinent and/or current Ecstasy users, and more particularly with problems in verbal memory (Cole \& Sumnall, 2003; Parrott, 2001). Research concerning spatial memory in MDMA users, however, has provided mixed results showing either the presence or absence of spatial memory deficits. It has been suggested that this discrepancy may be primarily related to the types of spatial memory task that have been employed in these studies, and that tasks requiring predominantly memory storage would be less vulnerable for MDMA than spatial memory tasks requiring storage as well as contextual processing of visuospatial cues (Wareing, Murphy, \& Fisk, 2004). However, there is little support for this notion when classifying spatial memory studies according to 3 distinguishable types of task.

First, there are studies using spatial span tasks in which subjects are required to repeat a previously seen or heard (Wareing, Fisk, \& Murphy, 2000) sequence, sometimes in combination with a visual decision task (Wareing, Fisk, Murphy, \& Montgomery, 2005; Wareing et al., 2004). Some between-group comparisons have revealed impairment of spatial memory in Ecstasy polydrug users compared to polydrug or nondrug controls (Verkes et al., 2001; Wareing et al., 2005; Wareing et al., 2004) whereas others did not (Gouzoulis Mayfrank et al., 2000; Morgan, 1998; Wareing et al., 2005; Wareing et al., 2000; Wareing et al., 2004). A second category includes studies using "delayed matching to sample tasks" or recognition tasks. In this type of task, the subject is required to compare a previously seen pattern or location with one that is shown after a certain delay, and respond by stating whether these two are the same or not. Some studies have found Ecstasy polydrug users to be impaired on this kind of tasks, as compared to a polydrug control group (Fox, Parrott, \& Turner, 2001; Verkes et al., 2001); whereas others did not (McCann, Mertl, Eligulashvili, \& Ricaurte, 1999; Semple, Ebmeier, Glabus, O'Carroll, \& Johnstone, 1999); Fox and colleagues (2002) also reported conflicting results on delayed matching to sample tasks. The third category comprises studies using "search tokens tasks". In this type of task, subjects are required to find hidden objects, one at a time, in an increasing number of boxes. Once a token or object is found in a certain place during one trial, the subject has to keep in mind not to return to that place. Fox and colleagues (2002) demonstrated that ecstasy users performed worse on this task, on the most difficult level, as compared to normal controls, but this finding conflicted with results from an earlier study (Semple et al., 1999). In other words, categorization of 
spatial memory tasks has not yet provided additional insight into the conflicting pattern of spatial memory deficits that has emerged in studies employing current and/or abstinent drug users.

Yet, the importance of task classifications may become more apparent when another confounding factor common to most studies in MDMA users can be controlled for, that is polydrug use. It is well known that MDMA-users also consume a variety of other drugs, most notably cannabis, alcohol, and cocaine (Rodgers et al., 2001; Scholey et al., 2004). All of these drugs have been associated with cognitive deficits (Dafters, Hoshi, \& Talbot, 2004; Verdejo Garcia, Lopez Torrecillas, Aguilar de Arcos, \& Perez Garcia, 2005), which in turn may have confounded results from studies on spatial memory function in MDMA users. One way to circumvent the problem of drug confounding is to study the effects of MDMA on spatial memory in a placebo-controlled, double-blind study that checks for and excludes concurrent drug use during memory testing by means of drug screening in blood or urine.

The present study is a double-blind, placebo-controlled study that aims to assess whether a single dose of MDMA affects spatial memory performance during the intoxication and withdrawal phase, and whether spatial memory performance after MDMA is task dependent. Two spatial memory tasks of varying complexity were used that required either storage of stimulus location alone (spatial memory task) or memory for location as well as processing of content or contextual information (change blindness task). Methylphenidate (Ritalin), a dopamine transporter blocker, was used as active control to see whether the effects of MDMA were mediated by dopamine and/ or serotonin.

\section{Methods}

\section{Design and treatments}

The study design was, double-blind, placebo-controlled, 3-way crossover, with balancing of the treatments. Testing took place on two consecutive days, in three separate testing periods, with a minimum washout period of 14 days in between. On the first day of each period, participants received either $75 \mathrm{mg}$ of MDMA, 20 $\mathrm{mg}$ of methylphenidate or placebo. MDMA powder was dissolved in $25 \mathrm{ml}$ bitter orange peel syrup and mixed with $200 \mathrm{ml}$ orange juice; and methylphenidate was administered as a pill. All treatments were identical in appearance. Participants received on each first day a solution and a pill in order to blind them and the researcher for the treatment. The second day was identical to the first day with the exception that no drugs or placebo were given. 


\section{Participants}

Twenty eight volunteers underwent medical examination. Three volunteers were excluded from participation as they did not meet inclusion or exclusion criteria. The remaining subjects passed the medical screening and entered the study. Seven subjects dropped-out prematurely either because of private matters (three cases) or because of repeated failure to abstain from drug use in between testing days as indicated by drug urine screens on test days (four cases). Eighteen subjects, nine male and nine female, aged in the range of 20 to 39 years (mean (SD) 26.22 (5.1)) completed all study treatments. They all had used MDMA before onset of the study. Lifetime use of MDMA varied from light ( $<30$ times) in 15 subjects to heavy (between 60-120 times) in three subjects. They were recruited by means of advertisements in local newspapers and the snowball technique. Potential candidates first underwent a telephonic screening. They were questioned about their drug use and medical condition and were given information about the study. They were sent a detailed brochure with information about the study procedure and two questionnaires for medical history and detailed history of drug use. Inclusion criteria were experience with MDMA use, free from psychotropic medication, good physical health, absence of major medical endocrine and neurological conditions, normal weight (BMI 18-28 kg/m²). Exclusion criteria were history of drug abuse (other than MDMA) or addiction, pregnancy or lactation, cardiovascular abnormalities (assessed by a standard 12-lead electrocardiogram (ECG)), excessive drinking (> 20 alcoholic consumptions), hypertension (diastolic $>100 \mathrm{mmHg}$; systolic $>170 \mathrm{mmHg}$ ), history of psychiatric or neurological disorders. A medical doctor checked the completed list, and upon approval, they were invited for a medical examination. Blood and urine samples were taken for examination and subjects underwent an ECG measurement. When they were found medically sound, they were contacted for participation. They were given a brochure with information about the study and a number of rules they had to obey during the period of the study and signed an informed consent to prove they read the information and agreed on it. They were paid upon completion of the testing periods for their participation. The study was performed in accordance with the 1975 declaration of Helsinki, adjusted in Edinburgh (2000) and was approved by the Medical Ethics Committee of the Academic Hospital of Maastricht and the University of Maastricht. A permit for obtaining, storing and administering MDMA was obtained from the Dutch drug enforcement administration. 


\section{Study procedure}

Before testing days, participants were familiarized with the tests on a training day. Participants were requested to abstain from any drug use 1 week prior to the medical examination until 14 days after the last testing day. They were asked not to use any caffeinated or alcoholic beverages 24 hours prior to testing and to get a normal night's sleep. Subjects were allowed to smoke but the amount of cigarettes was limited to two cigarettes during the whole testing period and smoking was not allowed right before cognitive testing.

Participants were screened for alcohol use in breath and for recent drug use in urine (THC/ opiates/ cocaine/ amphetamines/ methamphetamines) upon arrival at the testing facilities at $9 \mathrm{am}$. Women were given a pregnancy test. When the results on all of the tests were negative, subjects proceeded with a light breakfast and were given a sleep questionnaire to assess sleep complaints. One hour later subjects received drugs or placebo. One hour and a half after drug intake a blood sample was taken to determine the MDMA/MDA or ritalinic acid concentrations in blood plasma. Following blood sampling, subjects proceeded with the memory tests during the intoxication phase. Similar study procedures were followed when the subjects returned to the laboratory the following day to assess memory performance during withdrawal, i.e. between 25.5 and $26 \mathrm{~h}$ postdosing. An additional blood sample was taken every 11th day after drug administration to control for renal- and liver functioning. Subjects were instructed to report any adverse physical reactions that they might experience during drug intoxication. No serious adverse reactions emerged.

\section{Cognitive Measures}

Spatial memory task. The spatial memory task (Vermeeren et al., 1995) assesses short-term memory for spatial information. The subject is briefly shown a fixation point in the center of the computer screen. Shortly thereafter, a target appears at a random location for 500 milliseconds. The subjects' task is to memorize the location of the target and, using a computer mouse, relocate the cursor as accurately as possible over that position. The cursor appears either immediately upon target offset or after a delay of 2 or 4 seconds. The subject depresses the left mouse button to indicate that the cursor is at the recalled position of the target. The test consists of 75 trials, divided equally among the 3 response delays. The sequence of delays is random. Dependent variables are localization error and reaction time. 
Change blindness task. The change blindness task (Dornhoefer, Unema, \& Velichkovsky, 2002) assesses visual scene analysis and memory for changes in visuospatial information. The subject is presented a series of 100 photographs of traffic situations. Each photograph is shown for 3 seconds and followed by a 300milliseconds blank screen. During the blank period a fixation dot appears that subjects have to attend to. In the next 3 seconds the original photo reappears with or without a superimposed change in the traffic scene. Changes appear in $80 \%$ of the cases and half of those are either relevant or irrelevant to traffic (context of change). Fifty percent of the relevant and irrelevant changes occur at a position central or peripheral (location of change) relative to the position of the fixation dot during the blank period. Number of correct detections and reaction time are the dependent variables.

\section{Pharmacokinetics}

Blood samples were collected in 5-ml glass tubes containing sodium fluoride and potassium oxalate. After centrifugation at $4000 \mathrm{~g}$ for $10 \mathrm{~min}$, the resultant plasma was transferred to a clean tube and frozen at $-20^{\circ} \mathrm{C}$ until liquid chromatographymass spectrometry LC-MS/MS analysis. The limit of quantification for MDMA, MDA and ritalinic acid was $1 \mathrm{ng} / \mathrm{ml}$.

\section{Statistical analyses}

All statistical analyses were conducted by means of SPSS 11.5 for Windows. Each objective parameter was analyzed using GLM univariate repeated-measures procedures with Treatment (three levels) and Day of testing (two levels) as main within-subject factors. Additional factors were Response Delay (three levels) in the spatial memory task and Context of change (two levels) and Location of change (two levels) in the change blindness task. Separate contrast (drug vs. placebo) tests were conducted in case of a significant overall effect of Treatment or Treatment by Day. The alpha criterion level of significance was set at $p=0.05$.

\section{Results}

Spatial memory task. ANOVA revealed significant main effects of Treatment by Day $\left(\mathrm{F}_{2,34}=3.46 ; \mathrm{p}=0.043\right)$ and Response Delay $\left(\mathrm{F}_{2,34}=83.56 ; \mathrm{p}<0.001\right)$ on Localization Error. The latter effect reflects increasing localization error as a function of response delay. The former effect was further analyzed by means of separate drug-placebo contrasts on each day of testing. These revealed that MDMA significantly increased localization error on day $1\left(\mathrm{~F}_{1,17}=10.86 ; \mathrm{p}=0.004\right)$ 
as compared to placebo. No differences in localization error were apparent between drugs and placebo on day 2 (Fig. 1; Table 1).

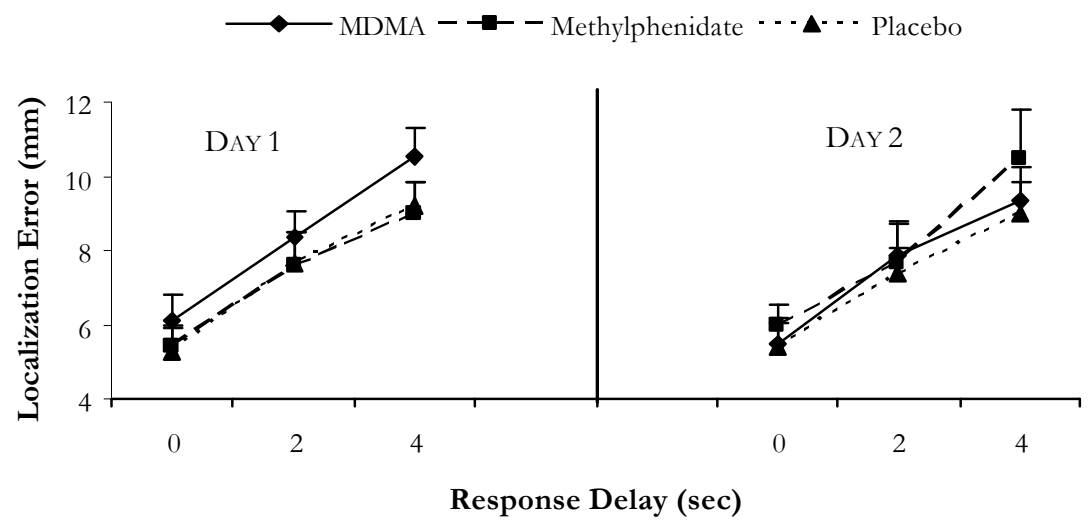

Figure 1. Mean (SE) Localization Error (mm), per Response Delay, for each drug condition on both test days.

Reaction Time showed significant main effects of Treatment by Day $\left(\mathrm{F}_{2,34}=6.44\right.$; $\mathrm{p}=0.004)$, Day $\left(\mathrm{F}_{1,17}=4.46 ; \mathrm{p}=0.050\right)$ and Response Delay $\left(\mathrm{F}_{2,34}=3.42 ; \mathrm{p}=0.044\right)$. The interaction effect was further analyzed by means of separate drug-placebo contrasts on each day of testing. These showed that MDMA significantly reduced Reaction Time on day $1\left(\mathrm{~F}_{1,17}=7.92 ; \mathrm{p}=0.012\right)$ as compared to placebo. No differences in Reaction Time were apparent between drugs and placebo on day 2 (Fig. 2; Table 1).

An additional multiple linear regression analysis was conducted to determine whether other factors independently correlated with Localization Error in the spatial memory task. The regression analysis was conducted on individual trial data rather than the subject means in order to achieve maximal statistical power. Each repetition of the spatial memory tests generated 75 individual trials. The total number of trials that entered the equation was determined by the number of subjects $\mathrm{x}$ the number of treatments $\mathrm{x}$ the number of individual trials in each spatial memory test repetition. Selected factors were Reaction Time, Drug (MDMA or placebo), Response Delay, Trial number and Localization Error. Results showed a significant positive correlation of the factors Drug and Response Delay with Localization Error. Together, these 2 factors significantly explained about $8 \% \quad\left(\mathrm{R}=0.289 ; \mathrm{R}^{2}=0.083\right)$ of the variance in Localization Error $\left(\mathrm{F}_{3,2699}=102.846 ; \mathrm{p}<0.001\right)$. Reaction Time was not correlated with localization error. 


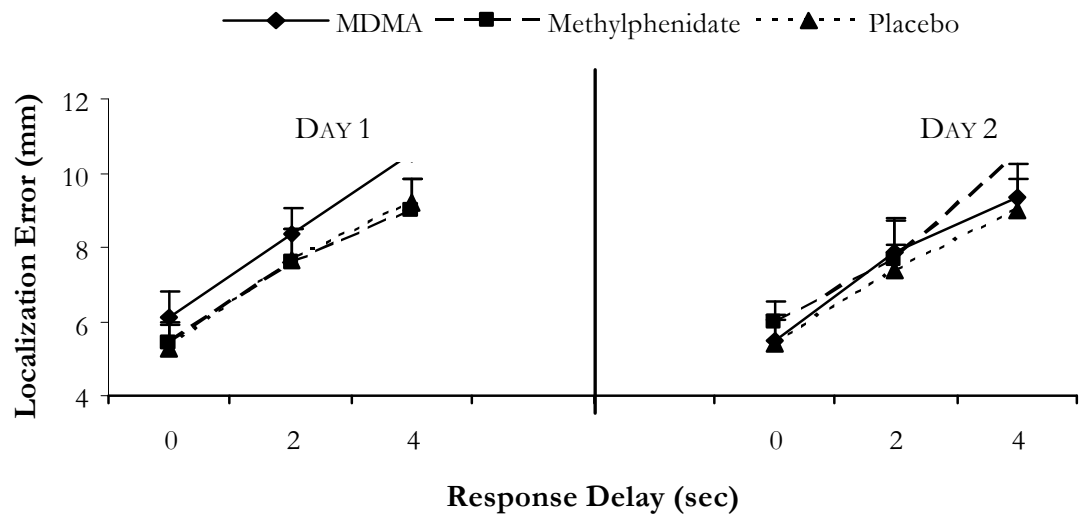

Figure 2. Mean (SE) Reaction Time (ms), per Response Delay, for each drug condition on both test days.

Table 1. Summary of means (SE), and Treatment by Day interaction effects in the Spatial Memory Task. (Overall $D f=2,34$ )

\begin{tabular}{|c|c|c|c|c|c|c|}
\hline $\begin{array}{l}\text { Dependent } \\
\text { variables }\end{array}$ & Day & Delay & \multicolumn{2}{|c|}{ MDMA } & TREATMENT & Placebo \\
\hline \multirow{6}{*}{$\begin{array}{l}\text { Localisation } \\
\text { Error }\end{array}$} & \multirow[t]{3}{*}{1} & 0 & \multicolumn{2}{|c|}{$6.14(0.67)$} & $5.39(0.53)$ & $5.28(0.65)$ \\
\hline & & 2 & \multicolumn{2}{|c|}{$8.36(0.66)$} & $7.59(0.88)$ & $7.63(0.90)$ \\
\hline & & 4 & \multicolumn{2}{|c|}{$10.49(0.84)$} & $8.96(0.88)$ & $9.21(0.64)$ \\
\hline & \multirow[t]{3}{*}{2} & 0 & \multicolumn{2}{|c|}{$5.51(0.69)$} & $5.94(0.58)$ & $5.43(0.63)$ \\
\hline & & 2 & \multicolumn{2}{|c|}{ 7.86(0.88) } & $7.67(1.01)$ & $7.36(0.69)$ \\
\hline & & 4 & \multicolumn{2}{|c|}{$9.34(0.91)$} & $10.46(1.32)$ & $9.01(0.82)$ \\
\hline \multirow{6}{*}{ Reaction Time } & \multirow[t]{3}{*}{1} & 0 & \multicolumn{2}{|c|}{$1659.67(77.70)$} & $176211(8812)$ & $1867.28(117.29)$ \\
\hline & & 2 & \multicolumn{2}{|c|}{$1602.66(83.44)$} & $1652.11(75.72)$ & $1803.72(98.16)$ \\
\hline & & 4 & \multicolumn{2}{|c|}{$1586.83(69.02)$} & $1734.50(85.23)$ & 1816.06(120.25) \\
\hline & 2 & 0 & \multicolumn{2}{|c|}{ 1822.67(119.50) } & $1842.44(124.50)$ & $1772.56(99.89)$ \\
\hline & & 2 & \multicolumn{2}{|c|}{$1715.44(96.52)$} & $\begin{array}{l}1042.44(124.50) \\
1789.78(92.26)\end{array}$ & $1748.22(103.34)$ \\
\hline & & 4 & \multicolumn{2}{|c|}{$1813.44(123.83)$} & $1828.89(116.11)$ & $1747.78(100.06)$ \\
\hline \multirow{2}{*}{$\begin{array}{l}\text { Dependent } \\
\text { variables }\end{array}$} & \multirow{2}{*}{ Day } & \multicolumn{3}{|c|}{$\begin{array}{c}\text { ANOVA } \\
\text { Overall (Treatment by Day) }\end{array}$} & \multicolumn{2}{|c|}{ Contrasts } \\
\hline & & & & $\mathrm{p}$ & MDMA/Placebo & $\mathrm{MP} / \mathrm{Placebo}$ \\
\hline \multirow{3}{*}{$\begin{array}{l}\text { Localisation } \\
\text { Error } \\
\text { Reaction Time }\end{array}$} & 1 & \multirow{2}{*}{\multicolumn{2}{|c|}{3.46}} & \multirow{2}{*}{0.043} & 0.004 & - \\
\hline & 2 & & & & - & - \\
\hline & $\begin{array}{l}1 \\
2\end{array}$ & \multicolumn{2}{|c|}{6.44} & 0.004 & $\begin{array}{c}0.012 \\
-\end{array}$ & - \\
\hline
\end{tabular}

Change blindness task. Reaction time performance in the change blindness task revealed overall effects of Context of change (relevant vs. irrelevant) $\left(F_{1,17}=\right.$ 184.01; $\mathrm{p}<0.001)$ and Location of change (central vs. peripheral) $\left(\mathrm{F}_{1,17}=51.15 ; \mathrm{p}<\right.$ $0.001)$ and Day $\left(F_{1,17}=23.55 ; \mathrm{p}<0.001\right)$. Number of Correct Detections was affected by Context of change $\left(\mathrm{F}_{1,17}=674.34 ; \mathrm{p}<0.001\right)$, Location of change $\left(\mathrm{F}_{1,17}=42.46 ; \mathrm{p}<0.001\right)$ and Day $\left(\mathrm{F}_{1,17}=23.55 ; \mathrm{p}<0.001\right)$. Changes relevant to traffic safety or driver performance were faster and more often detected than 
changes irrelevant to traffic. Changes in the central field of vision were faster and more frequently discovered than changes taking place in the peripheral field. Treatments did not affect performance. Interactions between Treatment and Content or Location of change also failed to reach statistical significance (Table 2).

Table 2. Summary of means (SE) in the Change Blindness Task. (Overall Df=2,34); $M P=$ Methylphenidate

\begin{tabular}{|c|c|c|c|c|c|c|}
\hline $\begin{array}{c}\text { Dependent } \\
\text { Variables }\end{array}$ & Day & $\begin{array}{l}\text { Context } \\
\text { of change }\end{array}$ & $\begin{array}{l}\text { Location } \\
\text { of change }\end{array}$ & MDMA & $\begin{array}{c}\text { TREATMENT } \\
\text { MP }\end{array}$ & Placebo \\
\hline \multirow{8}{*}{ \# correct } & \multirow[t]{4}{*}{1} & \multirow{2}{*}{ Irrelevant } & Central & $9.83(0.94)$ & $9.67(0.78)$ & 10.11(1.09) \\
\hline & & & Peripheral & $8.83(0.55)$ & $8.11(0.66)$ & $8.06(0.50)$ \\
\hline & & \multirow[t]{2}{*}{ Relevant } & Central & $15.94(0.47)$ & $15.94(0.45)$ & $15.33(0.52)$ \\
\hline & & & Peripheral & $15.89(0.59)$ & $16.61(0.30)$ & $16.39(0.33)$ \\
\hline & \multirow[t]{4}{*}{2} & \multirow[t]{2}{*}{ Irrelevant } & Central & $10.00(0.44)$ & $10.50(0.47)$ & $9.78(0.67)$ \\
\hline & & & Peripheral & $9.39(0.69)$ & $9.33(0.40)$ & $9.39(0.73)$ \\
\hline & & \multirow[t]{2}{*}{ Relevant } & Central & $17.56(0.33)$ & $17.22(0.31)$ & 17.44(0.37) \\
\hline & & & Peripheral & $16.61(0.32)$ & $15.56(0.47)$ & 16.11(0.39) \\
\hline \multirow{8}{*}{ RT } & \multirow{4}{*}{1} & \multirow[t]{2}{*}{ Irrelevant } & Central & $893.80(66.81)$ & $922.29(53.75)$ & $982.74(81.68)$ \\
\hline & & & Peripheral & $998.71(66.22)$ & $998.79(56.64)$ & $967.27(51.56)$ \\
\hline & & \multirow[t]{2}{*}{ Relevant } & Central & $658.64(27.81)$ & $681.80(27.10)$ & $723.90(43.24)$ \\
\hline & & & Peripheral & $756.58(29.73)$ & $766.57(37.58)$ & $822.92(38.48)$ \\
\hline & \multirow[t]{4}{*}{2} & \multirow[t]{2}{*}{ Irrelevant } & Central & $835.76(51.79)$ & 851.43(57.43) & $780.00(41.08)$ \\
\hline & & & Peripheral & $976.19(53.24)$ & $1004.49(54.66)$ & $929.80(51.46)$ \\
\hline & & \multirow[t]{2}{*}{ Relevant } & Central & $620.81(36.52)$ & $681.56(38.42)$ & $658.29(39.64)$ \\
\hline & & & Peripheral & $783.91(30.76)$ & 779.38(41.08) & $825.20(53.22)$ \\
\hline
\end{tabular}

\section{Pharmacokinetics}

Plasma concentrations of MDMA, MDA and ritalinic acid were determined 1.5 (day 1) and $25.5 \mathrm{~h}$ (day 2) post-drug. Concentrations of MDMA were on average (SD) 113.4 (37.4) and $11.6 \mathrm{ng} / \mathrm{ml}$ (9.3), respectively, on days 1 and 2 . Concentrations of MDA were on average 2.9 (0.9) and $2.1 \mathrm{ng} / \mathrm{ml}$ (1.2), respectively, on days 1 and 2. Concentrations of ritalinic acid were on average 95.9 (78.4) and $22.9 \mathrm{ng} / \mathrm{ml}(7)$, respectively, on days 1 and 2.

\section{Discussion}

An analysis of variance revealed that a single dose of MDMA caused subjects to perform worse on a simple spatial memory task during the intoxication phase. Subjects displayed larger localization errors and responded faster while under influence of MDMA. These effects were no longer visible during the withdrawal phase. To sort out whether changes in Reaction Time had contributed significantly to changes in Localization Error during MDMA intoxication, a multiple linear regression was conducted. It showed that Localization Error was not correlated with Reaction Time. MDMA treatment and Response Delay however were correlated and explained about $8 \%$ of the variance in Localization Error. Together, 
results from ANOVA and multiple regression indicate that MDMA is the primary factor underlying spatial memory impairment in the present study.

Contextual processing of visuospatial information in the change blindness task was not affected by treatment. Overall, changes relevant to traffic safety or driver performance were faster and more often detected than changes irrelevant to traffic. Changes in the central field of vision were faster and more frequently discovered than changes taking place in the peripheral field. However, spatial memory for changes in location or context was not affected by treatment. This seems to suggest that processing of information conveyed through a variety of spatial locations is not impaired during MDMA intoxication. An alternative explanation however might be that the change blindness task is not sensitive to drug effects. This cannot be totally excluded as this was the first study to apply a change blindness paradigm in a drug trial. However, the task was sufficiently sensitive to pick up on internal task manipulations (i.e. Content of change and Location of change), which indicates that the task is sensitive to external manipulation such as drug treatment as well. In sum, a single dose of MDMA impaired memory for location in a simple spatial memory task but did not affect processing of contextual information in a complex visuospatial memory task.

The absence of effects of the dopamine transporter blocker (methylphenidate) on performance is interesting. It indicates that MDMA's effect on spatial memory is not due to its action within the dopaminergic system. Though MDMA exerts its effects in the brain through a multitude of transmitter systems it is generally assumed that MDMA induced memory deficits that are related to changes in serotonergic function (Bolla, McCann, \& Ricaurte, 1998; McCann, Ridenour, Shaham, \& Ricaurte, 1994; Reneman et al., 2002; Verkes et al., 2001). The present data seem to support this general notion. Our data are also in line with results from a study of Luciana and colleagues (1998) who assessed the effects of a 5-HT agonist in a similar spatial memory paradigm as in the current study. Localization error increased after administering fenfluramine, a 5-HT agonist. This increased inaccuracy was not due to effects on motor performance, as indicated by the absence of a drug effect on latency. Our results mimic those of Luciana in that we also found increased inaccuracy independent of motor performance. Together, these results strengthen the notion that 5-HT suppletion following serotonergic drugs such as MDMA and fenfluramine produce spatial memory impairment. In addition, support for this serotonin hypothesis also comes from animal studies that have shown impairment of spatial memory after treatment with MDMA (Broening, Morford, Inman Wood, Fukumura, \& Vorhees, 2001; Sprague, Preston, Leifheit, \& 
Woodside, 2003) or d-fenfluramine (Morford, Inman Wood, Gudelsky, Williams, \& Vorhees, 2002) as well. Sprague and colleagues (2003) subsequently linked the MDMA-induced serotonergic lesions in the hippocampus to deficits seen in spatial memory. The overall conclusion that can be drawn from these animal studies is that 5-HT plays a direct or indirect role in the observed deficits in spatial memory after treatment with a 5 -HT agonist or MDMA.

Other researches studying the effects of methylphenidate on spatial memory performance however have shown memory performance improvement in young healthy volunteers (Elliott et al., 1997; Mehta et al., 2000). Elliott and colleagues (1997) showed that methylphenidate (20 and $40 \mathrm{mg}$ ) improved performance on a spatial span task and a search tokens task as compared to placebo, when the methylphenidate session preceded placebo sessions. These facilitating effects of the drug were determined by the familiarity with the task (novelty of the task) and consequently only showed when subjects received methylphenidate on the first session. Metha and colleagues (2000) showed that the size of effect of methylphenidate $(40 \mathrm{mg}$ ) on spatial memory correlated negatively with the baseline working memory capacity. This capacity was determined by performance on a span task, before administration of the drug. Subjects with lower working memory capacity showed the most improvement on a search tokens test in a positron emission tomography scanner after administration of methylphenidate as illustrated by a reduction in 'between search' errors and taskrelated reductions in $\mathrm{rCBF}$ when on methylphenidate. When comparing the results of these studies with the present results, a few comments can be made. First, cognitive tasks used in the present study were abundantly trained before testing took place, so in contrast to subjects in the study by Elliott and colleagues (1997) subjects were already familiar with the tasks. Second, it is likely that the working memory capacity of our subjects was rather high as shown by their excellent performance on the two spatial tasks during placebo treatment. Performance may have been close to maximal, leaving little space for measuring performance improvement when on methylphenidate. Third, both studies used another paradigm than the one used in the present study. A study mentioned earlier by Luciana and colleagues (1998) showed improvement of performance on the same task as used in the present study, after administration of a dopaminergic agent, bromocriptine. This improvement in performance was only visible after a long memory delay of 8 seconds and not at smaller memory delays as employed in the present study. 
It is noteworthy that spatial memory performance during placebo treatment as well as during MDMA withdrawal was close to maximal in the present study. Subjects in the present study were generally light recreational users of MDMA which may explain why their spatial memory performance appears relatively well as compared to some of the memory deficits that have been reported in abstinent users (Cole \& Sumnall, 2003; Parrott, 2001). Studies in abstinent users usually employ light users as well as heavy users of MDMA. Long term deficits during abstinence have repeatedly been associated with heavy MDMA use and may not have developed in light recreational users of MDMA as in the present study. The present data, therefore, cannot be taken to suggest that persisting spatial memory deficits will never occur during abstinence.

It is concluded that a single dose of MDMA impairs spatial memory for location in a simple spatial memory task but leaves processing of contextual visuospatial information in a more complex task intact.

\section{Acknowledgements}

We would like to thank Nele Samyn, Gert De Boeck and Marleen Laloup from NICC, Brussels, for analyzing MDMA and methylphenidate blood plasma samples.

This study was part of the IMMORTAL project, funded by the European Commission (Grant GMA1-2000-27043). 


\section{References}

Bolla, K. I., McCann, U. D., \& Ricaurte, G. A. (1998). Memory impairment in abstinent MDMA ("Ecstasy") users. Neurology, 51(6), 1532-1537.

Broening, H. W., Morford, L. L., Inman Wood, S. L., Fukumura, M., \& Vorhees, C. V. (2001). 3,4-methylenedioxymethamphetamine (ecstasy)-induced learning and memory impairments depend on the age of exposure during early development. Journal of neuroscience the official journal of the Society for Neuroscience, 21(9), 3228-3235.

Cole, J. C., \& Sumnall, H. R. (2003). Altered states: the clinical effects of Ecstasy. Pharmacology and Therapeutics, 98, 35-58.

Dafters, R. I., Hoshi, R., \& Talbot, A. C. (2004). Contribution of cannabis and MDMA ("ecstasy") to cognitive changes in long-term polydrug users. Psychopharmacology, 173(3-4), 405-410.

Dornhoefer, S. M., Unema, P. J., \& Velichkovsky, B. M. (2002). Blinks, blanks and saccades: how blind we really are for relevant visual events. Progress in brain research, 140, 119-131.

Elliott, R., Sahakian, B. J., Matthews, K., Bannerjea, A., Rimmer, J., \& Robbins, T. W. (1997). Effects of methylphenidate on spatial working memory and planning in healthy young adults. Psychopharmacology, 131(2), 196-206.

Fox, H. C., McLean, A., Turner, J. J., Parrott, A. C., Rogers, R., \& Sahakian, B. J. (2002). Neuropsychological evidence of a relatively selective profile of temporal dysfunction in drug-free MDMA ("ecstasy") polydrug users. Psychopharmacology, 162(2), 203-214.

Fox, H. C., Parrott, A. C., \& Turner, J. J. (2001). Ecstasy use: cognitive deficits related to dosage rather than self-reported problematic use of the drug. Journal of psychopharmacology Oxford, England, 15(4), 273-281.

Gouzoulis Mayfrank, E., Daumann, J., Tuchtenhagen, F., Pelz, S., Becker, S., Kunert, H. J., et al. (2000). Impaired cognitive performance in drug free users of recreational ecstasy (MDMA). Journal of neurology, neurosurgery, and psychiatry, 68(6), 719-725.

Luciana, M., Collins, P. F., \& Depue, R. A. (1998). Opposing roles for dopamine and serotonin in the modulation of human spatial working memory functions. Cerebral cortex New York, N.Y. 1991, 8(3), 218-226.

McCann, U. D., Mertl, M., Eligulashvili, V., \& Ricaurte, G. A. (1999). Cognitive performance in (+/-) 3,4-methylenedioxymethamphetamine (MDMA, "ecstasy") users: a controlled study. Psychopharmacology, 143(4), 417-425.

McCann, U. D., Ridenour, A., Shaham, Y., \& Ricaurte, G. A. (1994). Serotonin neurotoxicity after (+/-)3,4-methylenedioxymethamphetamine (MDMA; 
"Ecstasy"): a controlled study in humans. Neuropsychopharmacology official publication of the American College of Neuropsychopharmacology, 10(2), 129-138.

Mehta, M. A., Owen, A. M., Sahakian, B. J., Mavaddat, N., Pickard, J. D., \& Robbins, T. W. (2000). Methylphenidate enhances working memory by modulating discrete frontal and parietal lobe regions in the human brain. Journal of neuroscience the official journal of the Society for Neuroscience, 20(6), Rc65.

Morford, L. L., Inman Wood, S. L., Gudelsky, G. A., Williams, M. T., \& Vorhees, C. V. (2002). Impaired spatial and sequential learning in rats treated neonatally with Dfenfluramine. European journal of neuroscience, 16(3), 491-500.

Morgan, M. J. (1998). Recreational use of "ecstasy" (MDMA) is associated with elevated impulsivity. Neuropsychopharmacology official publication of the American College of Neuropsychopharmacology, 19(4), 252-264.

Parrott, A. C. (2001). Human psychopharmacology of Ecstasy (MDMA): A review of 15 years of empirical research. Human Psychopharmacology Clinical and Experimental, 16(8), 557-577.

Reneman, L., Endert, E., de Bruin, K., Lavalaye, J., Feenstra, M. G., de Wolff, F. A., et al. (2002). The acute and chronic effects of MDMA ("ecstasy") on cortical 5-HT2A receptors in rat and human brain. Neuropsychopharmacology official publication of the American College of Neuropsychopharmacology, 26(3), 387-396.

Rodgers, J., Buchanan, T., Scholey, A. B., Heffernan, T. M., Ling, J., \& Parrott, A. (2001). Differential effects of Ecstasy and cannabis on self-reports of memory ability: A web-based study. Vol 16(8), 619-625 URLJ: http://www.interscience.wiley.com/ipages/0885-6222/.

Scholey, A. B., Parrott, A. C., Buchanan, T., Heffernan, T. M., Ling, J., \& Rodgers, J. (2004). Increased intensity of Ecstasy and polydrug usage in the more experienced recreational Ecstasy/MDMA users: a WWW study. Addictive behaviors, 29(4), 743752.

Semple, D. M., Ebmeier, K. P., Glabus, M. F., O'Carroll, R. E., \& Johnstone, E. C. (1999). Reduced in vivo binding to the serotonin transporter in the cerebral cortex of MDMA ('ecstasy') users. British journal of psychiatry the journal of mental science, 175, 63-69.

Sprague, J. E., Preston, A. S., Leifheit, M., \& Woodside, B. (2003). Hippocampal serotonergic damage induced by MDMA (ecstasy): effects on spatial learning. Physiology and behavior, 79(2), 281-287.

Verdejo Garcia, A. J., Lopez Torrecillas, F., Aguilar de Arcos, F., \& Perez Garcia, M. (2005). Differential effects of MDMA, cocaine, and cannabis use severity on 
distinctive components of the executive functions in polysubstance users: a multiple regression analysis. Addictive behaviors, 30(1), 89-101.

Verkes, R. J., Gijsman, H. J., Pieters, M. S., Schoemaker, R. C., de Visser, S., Kuijpers, M., et al. (2001). Cognitive performance and serotonergic function in users of ecstasy. Psychopharmacology, 153(2), 196-202.

Vermeeren, A., Jackson, J. L., Muntjewerff, N. D., Quint, P. J., Harrison, E. M., \& O'Hanlon, J. F. (1995). Comparison of acute alprazolam (0.25, 0.50 and $1.0 \mathrm{mg})$ effects versus those of lorazepam $2 \mathrm{mg}$ and placebo on memory in healthy volunteers using laboratory and telephone tests. Psychopharmacology, 118(1), 1-9.

Wareing, M., Fisk, J. E., Murphy, P., \& Montgomery, C. (2005). Visuo-spatial working memory deficits in current and former users of MDMA ('ecstasy'). Human psychopharmacology, 20(2), 115-123.

Wareing, M., Fisk, J. E., \& Murphy, P. N. (2000). Working memory deficits in current and previous users of MDMA ('ecstasy'). British journal of psychology, 91(Pt 2), 181-188.

Wareing, M., Murphy, P. N., \& Fisk, J. E. (2004). Visuospatial memory impairments in users of MDMA ('ecstasy'). Psychopharmacology, 173(3-4), 391-397. 


\title{
Chapter 4
}

\section{MEMORY AND MOOD DURING THE NIGHT AND IN THE MORNING AFTER REPEATED EVENING DOSES \\ OF MDMA}

\author{
Kuypers, K.P.C., Wingen, M., and Ramaekers, J.G.
}

Background. Previously it has been shown that MDMA causes memory impairment during daytime testing. However, MDMA is usually taken in the evening or during the night. In addition, it is known that sleep deprivation also causes memory impairment. Aim. The present study aimed to assess whether evening doses of MDMA added to, or interacted with the memory impairment due to sleep deprivation. Methods. Fourteen healthy subjects participated in a double-blind, placebo-controlled, 2-way cross-over study. Treatments consisted of MDMA $75 \mathrm{mg}$ and $50 \mathrm{mg}$, administered at $8 \mathrm{PM}$ and $12 \mathrm{PM}$ respectively, or double placebo. Memory tests and subjective measures of mood were conducted at baseline and three times post dosing i.e. at 6.30 PM, 9.30 PM, 1.30 AM and 7 AM, respectively -1.5 $\mathrm{h}, 1.5 \mathrm{~h}, 5.5 \mathrm{~h}$ and $11 \mathrm{~h}$ relative to drug intake (1 ${ }^{\text {st }}$ dose). Results. Memory performance detoriated progessively over time as a function of sleep loss, independent of treatment. MDMA added to this impairment as indicated by a significant main effect of MDMA on verbal and spatial memory performance. Mood ratings and response speed revealed an MDMA by Time interaction. After administration of MDMA, response speed improved and feelings of vigor, friendliness, elation, anxiety, confusion, arousal and positive mood increased in magnitude during the night, while all these parameters returned to placebo-like levels on the final morning session. Conclusion. It is concluded that evening doses of MDMA selectively impair memory performance, and that this impairment is additional to the effect of sleep deprivation on memory performance. 
56 | CHAPTER 4 


\section{Introduction}

Ecstasy has been frequently associated with cognitive impairment. Several studies have shown that abstinent ecstasy users display cognitive deficits, such as verbal, spatial and working memory deficits (reviews (Cole \& Sumnall, 2003; Parrott, 2001)). A recent longitudinal study showed that impairment of verbal memory persisted for longer than 2.5 years after quitting the use of ecstasy (Thomasius et al., 2006). However, the possibility exists that other factors contributed to these memory deficits such as the existence of pre-morbid group differences and polydrug use. In addition, even when groups of ecstasy users were compared with polydrug users groups, the pattern of drug use, apart from ecstasy, might differ (Gouzoulis Mayfrank \& Daumann, 2006).

Recently, a direct and selective pharmacological link between memory impairment and MDMA intoxication has been established in two acute, placebocontrolled within-subject studies (Kuypers \& Ramaekers, 2005; Kuypers \& Ramaekers, 2007). Verbal memory was impaired in subjects after administration of MDMA compared with placebo as demonstrated by a decrement in immediate and delayed recall in a word-learning task. While showing a normal learning curve over successive trials when under influence of MDMA, subjects learned less compared with placebo. Spatial memory was also impaired after an acute dose of MDMA, as shown by larger localization error during target localization in a simple spatial memory task. Other skills like syntactic reasoning remained unimpaired under the influence of MDMA.

Characteristic of these studies was that subjects were tested during daytime after administration of a single dose of MDMA. However, ecstasy users typically take one or more tablets during the evening or night (Farre et al., 2004) and this is is often accompanied by insufficient rest. Sleep deprivation (stressor) interferes with allostasis, a process which serves to maintain a balance between several mediators (e.g. glucocorticoids, cytokines) in the normal functioning body. Moreover, it has been shown that even very limited sleep restriction (i.e. 6 hours sleep/night) can cause an imbalance in these mediators (e.g cortisol) and can consequently damage structures implicated in important functions e.g. memory (McEwen, 2006; Parrott, 2006).

Parrott and Lasky (1998) who studied the acute and sub-acute effects of ecstasy in combination with other drugs on memory in a naturalistic setting, showed that verbal memory was impaired $2-8$ or $8-16$ hours after drug intake, compared with two control groups (Parrott \& Lasky, 1998). This is in line with results of our previous study, showing impairing effects of a single dose of MDMA 
on memory (Kuypers \& Ramaekers, 2005). However, Parrott and Lasky (1998) showed that the impairing effects lasted up to 7 days whereas the memory impairment in Kuypers and Ramaekers' study was 25.5 hours after MDMA administration not present. This prolonged presence of memory deficits in Parrott and Lasky's study could be due to additional factors like the combined use of several drugs and/or disturbed sleep pattern. It is therefore possible that supplementary stressors like sleep deprivation and repeated dosing magnify memory deficits caused by MDMA.

The aim of the present study was to assess whether evening doses of MDMA added to or interacted with the memory impairment due to sleep deprivation. The design was double blind, placebo-controlled and two-way crossover. Subjects received two doses of MDMA, separated by an interval of four hours, and were tested four times (75 minutes) throughout the evening and night i.e. at 6.30 PM, 9.30 PM, 1.30 AM and 7 AM. Three cognitive tests assessing working memory (e.g. verbal, spatial) and a subjective measure of mood state were included. This mood questionnaire was included to provide additional information about the subjective feelings the subject experienced during the experimental sessions next to the objective measures of impairment.

\section{Methods}

\section{Design and treatments}

The study was conducted according to a double-blind, placebo-controlled, twoway within subject design. The treatments consisted of two subsequent doses of MDMA (75 mg and $50 \mathrm{mg}$ ) and double placebo. According to the annual report (2005) of the 'Drugs information and monitoring system' (DIMS) of the Netherlands, average recreational doses of MDMA may vary between 0 and 200 $\mathrm{mg}$, taken as 1 or 2 pills per evening (DIMS, 2005). Doses up to $150 \mathrm{mg}$ have previously been studied in acute studies. It was shown that side effects were limited with doses up to $125 \mathrm{mg}$ but appeared to be more frequent with $150 \mathrm{mg}$ doses (Cami et al., 2000; de la Torre et al., 2000). Dose administration was separated in time by 4 hours, at respectively 8 PM and 12 PM. A four-hour interval was chosen because subjects tend to take an additional dose when the subjective effects have worn off and that is approximately after 4 hours (Cami et al., 2000; Hammersley, 1999). Placebo and MDMA were administered orally in identically appearing formulations. MDMA was administered as a $25 \mathrm{ml}$ solution in bitter orange peel syrup; placebo was given as $25 \mathrm{ml}$ bitter orange peel syrup; both were mixed with $200 \mathrm{ml}$ orange or apple juice. 


\section{Participants}

Twenty volunteers underwent medical examination. Two volunteers were excluded from participation as they did not meet inclusion or exclusion criteria. The remaining subjects passed the medical screening and entered the training. Four subjects dropped-out prematurely either because of private matters (3x) or because of not feeling well on the first test day after placebo treatment (1x).

In total, fourteen subjects (7 male and 7 female) aged in the range of 19-33 years (mean (SD) 22.93 (3.75)) completed all study treatments. They all had used MDMA prior to onset of the study. Lifetime use of MDMA varied from light $(<25$ times) in 9 subjects to moderate (between 30-35 times) in five subjects.

Subjects were recruited by means of advertisements in local newspapers and the snowball technique. Potential candidates were questioned about their drug use and medical condition and were given information about the study. Thereupon they were sent a detailed brochure with information about the study procedure and two questionnaires for medical history and detailed history of drug use. Inclusion criteria were experience with MDMA use, free from psychotropic medication, good physical health, absence of major medical endocrine and neurological conditions, normal weight (Body Mass Index 18-28 kg/m²). Exclusion criteria were history of drug abuse (other than MDMA) or addiction, pregnancy or lactation, cardiovascular abnormalities (assessed by a standard 12-lead electrocardiogram (ECG)), excessive drinking (> 20 alcoholic consumptions per week), hypertension (diastolic >100 mmHg; systolic $>170 \mathrm{mmHg}$ ), history of psychiatric or neurological disorders. A medical doctor checked the completed list, and upon approval, subjects were invited for a medical examination. Blood and urine samples were taken for examination and subjects underwent an ECG measurement. When medically fit, they were contacted and received a brochure with information about the study and a number of rules they had to obey during the period of the study. Subjects signed an informed consent to prove they read the information and agreed on it. They were paid upon completion of the testing periods for their participation. The study was performed in accordance with the 1975 declaration of Helsinki, adjusted in Edinburgh (2000) and was approved by the Medical Ethics Committee of the Academic Hospital of Maastricht and the University of Maastricht. A permit for obtaining, storing and administering MDMA was obtained from the Dutch drug enforcement administration. 


\section{Study procedure}

Before testing days, participants were familiarized with the tests on a training day. Participants were requested to abstain from any drug use 1 week before the medical examination until 14 days after the last testing day. They were asked not to use any caffeinated or alcoholic beverages $24 \mathrm{~h}$ before testing and to get a normal night's sleep. Participants were screened for alcohol use in breath and for recent drug use in urine (THC/opiates/cocaine/amphetamines/methamphetamines) upon arrival at the testing facilities at 6 PM. Women were given a pregnancy test. When tests were negative, the subjects received a light meal and were given a sleep questionnaire to assess sleep complaints and a mood questionnaire (POMS) to assess their mood state. Cognitive tests and POMS were assessed four times during the evening and night at 6.30 PM (baseline measure), 9.30 PM, $1.30 \mathrm{AM}$ and $7 \mathrm{AM}$; respectively 1.5 hours pre-dosing and, $1.5,5.5$ and 11 hours post-dosing ( 1 st dose). A test day ended at 8.15 AM. A blood sample was collected before each test session, except at baseline, to determine MDMA and MDA concentrations in blood serum. Participants were kept awake the whole night (e.g. they played party games, watched TV and/or listened to music). Testing days were minimally separated by 7 days.

\section{Cognitive assessments}

Spatial memory task. In the spatial memory task (Vermeeren et al., 1995) subjects were briefly shown a fixation point in the center of the computer screen. Shortly thereafter, a target appeared at a random location for 500 milliseconds. Subjects had to memorize the location of the target and, using a computer mouse, relocate the cursor as accurately as possible over that position. The cursor appeared either immediately upon target offset or after a delay of 2 or 4 seconds. Subjects depressed the left mouse button to indicate that the cursor was at the recalled position of the target. The task consisted of 75 trials, divided equally among the 3 response delays, and the sequence of delays was random. Short-term memory for spatial information was assessed. Dependent variables were localization error and reaction time.

Sternberg memory scanning task. In the memory scanning test (Sternberg, 1966) subjects were briefly shown a set of unrelated consonants they had to memorise. This was called the "memory set". Thereafter a series of 90 letters was displayed on a computer screen. Subjects had to respond to each letter as rapidly as possible by pressing either a 'yes' or 'no' button to indicate whether or not the letter belonged 
to the memory set. This task was performed with memory sets consisting of 1,2 and 4 letters respectively. Half of the presented letters were part of the memory set. The task assessed speed of memory search or retrieval of verbal stimuli from memory. The dependent variable was the mean of the response time for correct responses on targets and non targets.

Star counting task. The star counting task (de Jong \& Das Smaal, 1995) consisted of nine trials. Each trial consisted of a matrix of stars with plus and minus signs in between. Subjects had to start adding and subtracting stars from a given number which was shown in the upper left corner. The plus and the minus signs indicated whether stars should be added to, or subtracted from the total score. This task required the control of a very simple process, namely counting (forward and backward), known to involve working memory. The dependent variable was the number of correct calculations.

\section{Subjective assessments}

Two subjective measurements were taken: i.e. the Groninger Sleep Scale (GSS) and the Profile of Mood States (POMS). The GSS (Mulder-Hajonides van der Meulen, Wijnberg, Hollanders, DeDiana, \& Hoofdakker, 1980) assessed sleep quality and quantity (hours of sleep). It consists of fifteen dichotomous questions about sleep complaints and an open question concerning the duration of sleep. The POMS (de Wit, Enggasser, \& Richards, 2002) is a self-assessment mood questionnaire with 72 five point-Likert scale items, representing eight mood states; i.e. Anxiety, Depression, Anger, Vigor, Fatigue, Confusion, Friendliness and Elation. Two extra scales were derived, i.e. Arousal ((Anxiety + Vigor) $-($ Fatigue + Confusion $))$ and Positive mood (Elation - Depression). The subject had to indicate in how far these items were representing his/her mood.

\section{Pharmacokinetic assessments}

Blood samples were taken at $1.5 \mathrm{~h}, 5.5 \mathrm{~h}$ and $11 \mathrm{~h}$ post dosing (1st dose), centrifuged immediately at 4,000xg for $10 \mathrm{~min}$ and the corresponding serum samples were subsequently frozen at minus $20{ }^{\circ} \mathrm{C}$ until analysis for pharmacokinetic assessment. MDMA and MDA concentrations were determined using an integrated on-line SPE-LC-MS system (Symbiosis Pharma, Spark Holland) with tandem mass spectrometric detection (Quattro Premier, Waters Corportation) using deuterated analogues for internal standardization. Quantification limits were $5.0 \mathrm{ng} / \mathrm{ml}$ for MDMA and $2.5 \mathrm{ng} / \mathrm{ml}$ for MDA. 


\section{Statistical analyses}

All statistical analyses were conducted by means of SPSS 11.5 for Windows. T-tests were applied to baseline data to check for baseline differences between treatments. All cognitive variables entered GLM univariate repeated measures procedures with MDMA (2 levels) and Time (4 levels) as main within-subject factors. Additional factors were Response Delay (3 levels) in the spatial memory task and Memory Load (3 levels) in the Sternberg memory scanning task. Separate contrast (drug vs placebo) tests were conducted in case of a significant overall effect of MDMA or MDMA by Session. Subjective parameters of the POMS and Groninger sleep questionnaire were analysed by means of nonparametric Wilcoxon rank tests. The alpha criterion level of significance was set at $p=0.05$. In case of significant main effects of MDMA or Time, post-hoc analyses were conducted and alpha was then corrected for multiple comparisons.

\section{Results}

There were no statistically significant differences between baseline scores of the cognitive tasks. In the subjective tasks, there was a baseline difference on the fatigue scale and therefore difference scores for the fatigue scale were calculated.

\section{Cognitive assessments}

Spatial memory task. Analysis revealed significant main effects of MDMA $\left(\mathrm{F}_{1,13}=\right.$ 6.152; $\mathrm{p}=0.028)$, Time $\left(\mathrm{F}_{3,39}=13.207 ; \mathrm{p}<0.001\right)$, Response Delay $\left(\mathrm{F}_{2,26}=130.99\right.$; $\mathrm{p}<0.001)$ and MDMA by Response Delay $\left(\mathrm{F}_{2,26}=3.41 ; \mathrm{p}=0.048\right)$ on Localization Error. Localization errors were on average higher while subjects were under influence of MDMA (10.38) compared with placebo (8.91). Further analysis of the Time effect revealed that localization error gradually increased as a function of Time $\left(\mathrm{F}_{1,13}=20.87 ; \mathrm{p}=0.001\right)$ (Fig 1). This elevation of Localization Error over time was independent of MDMA as demonstrated by the absence of a significant MDMA by Time interaction (Table 1).

Reaction Time showed significant effects of MDMA $\left(\mathrm{F}_{1,13}=9.62 ; \mathrm{p}=\right.$ 0.008), Time $\left(\mathrm{F}_{3,39}=9.43 ; \mathrm{p}=0.002\right)$ and MDMA by Time $\left(\mathrm{F}_{3,39}=4.48 ; \mathrm{p}=0.023\right)$. Subjects responded faster, particularly on session 2 and 3 , under influence of MDMA compared with placebo (quadratic contrast, $\mathrm{F}_{1,13}=9.38 ; \mathrm{p}=0.009$ ) (Fig 2; Table 1). 


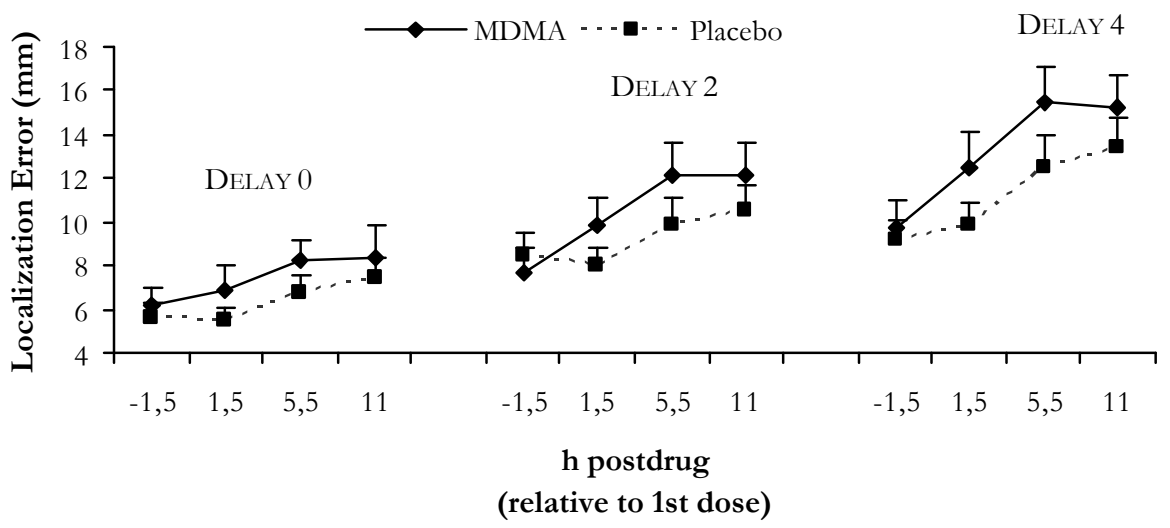

Figure 1. Localization Error ( $\mathrm{mm}$ ) in the Spatial memory task, per session and Response Delay (-1.5, 1.5, 5.5 and 7 hours post-drug correspond respectively with 6.30 pm, 9.30 pm, 1.30 am and 7 am).

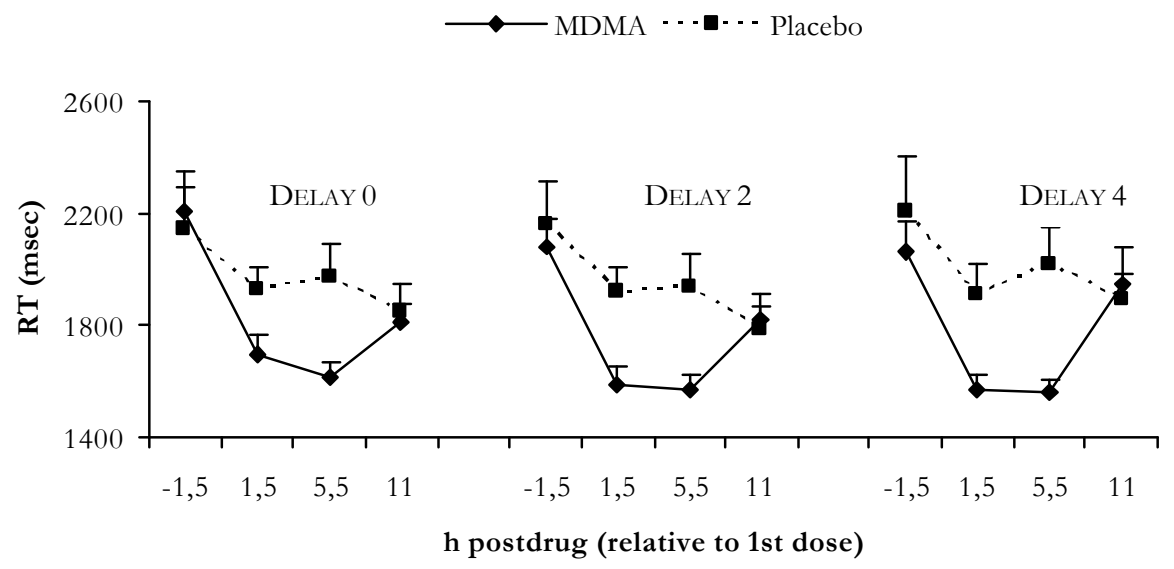

Figure 2. Reaction Time (msec) in the Spatial memory task, per session and Response Delay. (-1.5, 1.5, 5.5 and 7 hours post-drug corresponds respectively with 6.30 pm, 9.30 pm, 1.30 am and 7 am).

An additional multiple regression analysis was conducted to determine whether other factors independently correlated with Localization Error in the spatial memory task. The analysis was conducted on individual trial data rather than the subjects' means in order to achieve maximal statistical power. The seven predictors that initially entered the model were subject number (1 to 14), MDMA (placebo, MDMA), Time (2, 3), Trial number (1 to 75$)$, Response Delay $(0,2,4)$, distance 
from fixation point (Circle) 1 to 5)) and Reaction Time. The stepwise regression procedure removed eventually two predictors (subject number and Reaction Time). The resulting model yielded five predictors i.e. Delay, Circle, MDMA, Time and Trial Number. Together, these predictors explained about $14 \%(R=0.378$; $\left.R^{2}=0.143\right)$ of the variance in Localization Error $\left(F_{5,4194}=139.76, p<0.001\right)$ Reaction Time was not correlated with Localization Error.

Sternberg memory scanning task. Analysis revealed significant effects of MDMA $\left(\mathrm{F}_{1,13}=\right.$ 5.75; $\mathrm{p}=0.032)$, Time $\left(\mathrm{F}_{3,39}=10.35 ; \mathrm{p}<0.001\right)$, and Memory Load $\left(\mathrm{F}_{2,26}=245.19\right.$; $\mathrm{p}<0.001)$ on reaction time. Under influence of MDMA, subjects responded 21.85 msec on average slower compared with placebo (Fig 3). Reaction time increased as function of time after drug administration (linear contrast; $\mathrm{F}_{1,13}=16.10 ; \mathrm{p}=0.001$ ). There was no significant MDMA by Time interaction (Table 1).

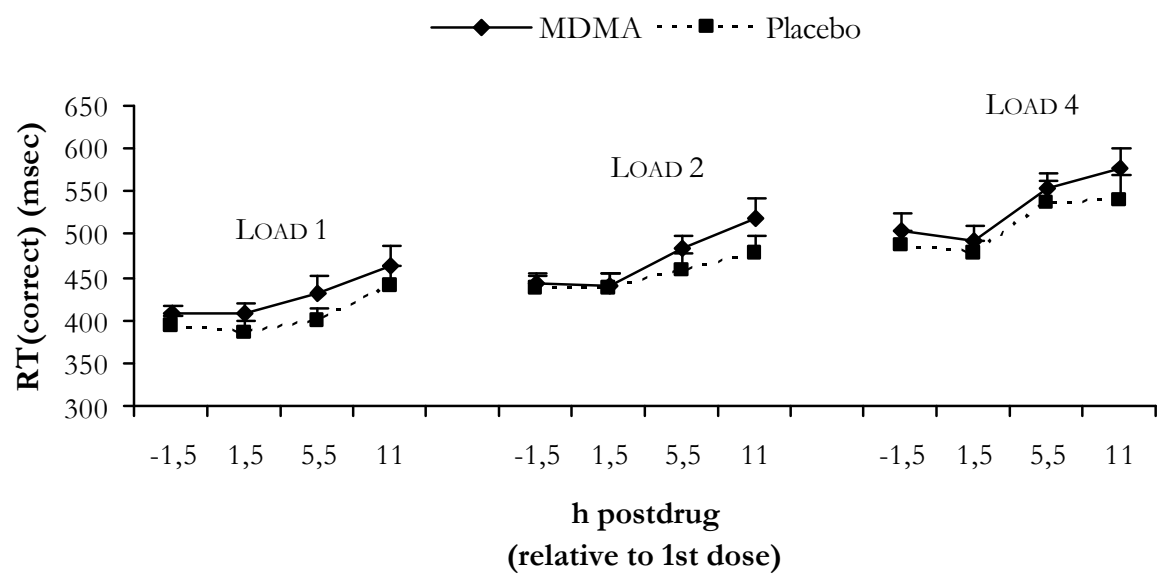

Figure 3. Reaction Time (msec) in the Sternberg memory scanning task, per session and Memory Load. (1.5, 1.5, 5.5 and 7 hours post-drug corresponds respectively with 6.30 pm, 9.30 pm, 1.30 am and 7 am).

Star counting task. Analysis revealed significant effects of MDMA $\left(\mathrm{F}_{1,13}=6.45 ; \mathrm{p}=\right.$ $0.025)$ and MDMA by Time $\left(\mathrm{F}_{3,39}=2.85 ; \mathrm{p}=0.050\right)$ on number of correct calculations. Subjects had a lower score in the MDMA condition compared with placebo. Decrements were particularly noteworthy during the second and third session, but not in the final morning session. There were no significant effects of MDMA, Time, or MDMA by Time on Reaction Time (Fig 4; Table 1). 


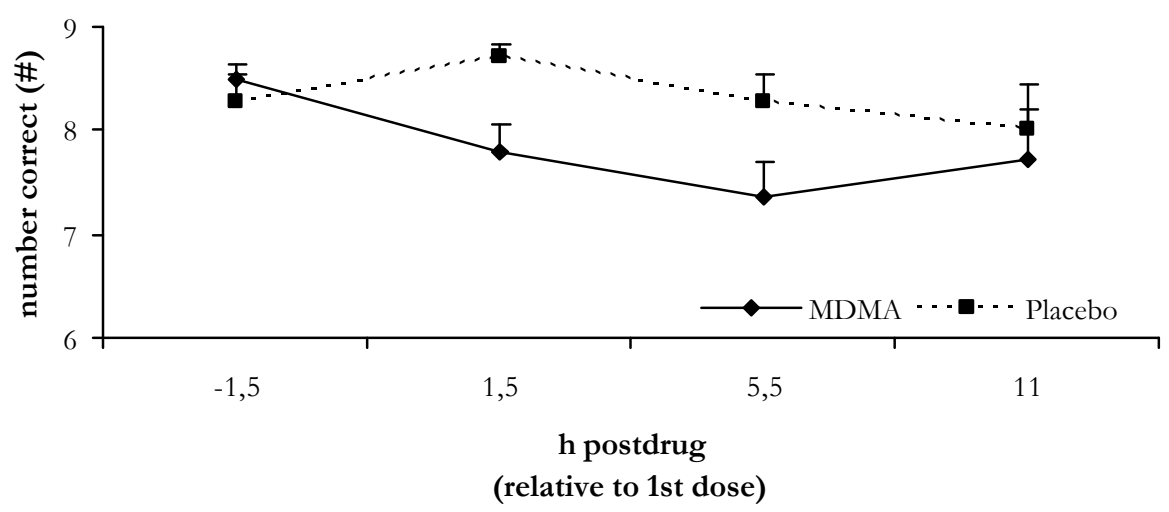

Figure 4. Number of correct calculations in the star counting task, per session. (-1.5, 1.5, 5.5, and 7 hours post-drug corresponds respectively with $6.30 \mathrm{pm}, 9.30 \mathrm{pm}, 1.30$ am and 7 am).

\section{Subjective assessments}

Groninger sleep scale. There were no significant main effects of MDMA on Sleep Quantity or Quality scores on the evening prior to testing. Subjects slept on average $8.03 \mathrm{~h}$ (SE 0.30) and had an average score of 2.64 (SE 0.74) on the sleep complaints questionnaire.

POMS. Analysis revealed effects of MDMA on 7 out of 10 scales of the POMS. Subjects showed increased scores during session 2 and 3 on Anxiety, Vigor, Confusion (only session 2), Friendliness, Elation, Arousal and Positive mood under influence of MDMA compared with placebo. There were no significant MDMA effects on measures of Depression, Fatigue and Anger (Table 2).

\section{Pharmacokinetic assessments}

Blood serum concentrations of MDMA were on average (SD) 171.79 (54.63), 298.25 (65.38) and 199.27 (51.90), respectively 1.5, 5.5 and 11 hours post-dosing ( $1^{\text {st }}$ dose). The higher concentrations in the second and third measurement are in agreement with the expected levels after administration of a $2^{\text {nd }}$ dose, 4 hours after the first one. Concentrations of MDA were on average (SD) 6.35 (3.04), 17.84 (7.48), and 21.94 (9.17), respectively 1.5, 5.5 and 11 hours post-dosing (1 ${ }^{\text {st }}$ dose). 
66 | CHAPTER 4

Table 1. Mean scores (SE) of cognitive parameters in three memory tasks. Ses= Session; Del= Delay

\begin{tabular}{|c|c|c|c|c|c|c|c|}
\hline & & & TRE & TMENT & & ANOVA & \\
\hline Task & Ses & Del & MDMA & Placebo & MDMA & Ses & $\begin{array}{c}\text { MDMA* } \\
\text { Ses }\end{array}$ \\
\hline Spatial Mei & DRY T & & & & & & \\
\hline Localization & 1 & 0 & $6.17(.80)$ & $5.55(.71)$ & & & \\
\hline Error & & 2 & $7.62(1.22)$ & $8.44(1.08)$ & & & \\
\hline & & 4 & $9.75(1.26)$ & $9.17(.92)$ & & & \\
\hline & 2 & 0 & $6.90(1.06)$ & $5.45(.67)$ & & & \\
\hline & & 2 & $9.90(1.20)$ & $8.00(.80)$ & & & \\
\hline & & 4 & $12.53(1.51)$ & $9.82(1.11)$ & 0.028 & 0.000 & - \\
\hline & 3 & 0 & $8.23(.99)$ & $6.75(.78)$ & $0.0 \angle 0$ & 0.000 & - \\
\hline & & 2 & $12.16(1.48)$ & $9.88(1.25)$ & & & \\
\hline & & 4 & $15.52(1.60)$ & $12.47(1.57)$ & & & \\
\hline & 4 & 0 & $8.36(1.46)$ & $7.42(.99)$ & & & \\
\hline & & 2 & $12.16(1.46)$ & $10.53(1.11)$ & & & \\
\hline & & 4 & $15.23(1.48)$ & $13.41(1.42)$ & & & \\
\hline Reaction & 1 & 0 & $2207.50(138.12)$ & $2143.29(151.41)$ & & & \\
\hline Time & & 2 & 2078.14(99.78) & $2160.50(155.11)$ & & & \\
\hline & & 4 & $2063.50(103.25)$ & $2204.64(201.65)$ & & & \\
\hline & 2 & 0 & $1691.86(78.70)$ & $1926.71(81.01)$ & & & \\
\hline & & 2 & $1590.07(56.87)$ & $1917.57(93.92)$ & & & \\
\hline & & 4 & $1565.79(59.50)$ & 1914.21(102.76) & & & \\
\hline & 3 & 0 & $1613.07(59.14)$ & $1972.93(117.66)$ & 0.008 & 0.002 & 0.023 \\
\hline & & 2 & $1567.00(57.06)$ & $1936.14(117.08)$ & & & \\
\hline & & 4 & $1559.57(43.54)$ & 2020.71(131.43) & & & \\
\hline & 4 & 0 & $1814.29(56.50)$ & $1845.50(100.68)$ & & & \\
\hline & & 2 & $1819.29(95.13)$ & $1786.79(75.22)$ & & & \\
\hline & & 4 & 1941.86(142.94) & $1895.71(82.08)$ & & & \\
\hline STERNBERG I & EMOI & TASK & & & & & \\
\hline & & Load & & & & & \\
\hline $\mathrm{RT}($ Correct) & 1 & 1 & $407.53(8.34)$ & $392.98(13.32)$ & & & \\
\hline & & 2 & $443.62(11.08)$ & $436.40(16.19)$ & & & \\
\hline & & 4 & $505.43(18.40)$ & $487.21(16.91)$ & & & \\
\hline & 2 & 1 & $406.63(13.02)$ & $385.13(14.28)$ & & & \\
\hline & & 2 & $440.25(13.95)$ & $437.22(18.72)$ & & & \\
\hline & & 4 & $492.89(18.46)$ & $476.66(17.19)$ & 0032 & 0001 & - \\
\hline & 3 & 1 & 432.13(19.01) & $398.29(15.61)$ & 0.032 & 0.001 & - \\
\hline & & 2 & $483.48(15.96)$ & $457.35(19.14)$ & & & \\
\hline & & 4 & $554.04(17.53)$ & $535.92(25.32)$ & & & \\
\hline & 4 & 1 & $462.08(24.34)$ & $439.79(23.91)$ & & & \\
\hline & & 2 & $519.07(24.09)$ & $477.20(21.82)$ & & & \\
\hline & & 4 & $578.53(20.63)$ & $539.28(27.81)$ & & & \\
\hline STAR COUNT & $\mathrm{NGT}$ & & & & & & \\
\hline $\mathrm{RT}$ (Correct) & 1 & & $826.30(2045.38)$ & $32966.74(2473.89)$ & & & \\
\hline & 2 & & $992.82(2118.03)$ & $30839.04(1663.36)$ & - & - & - \\
\hline & 3 & & 644.16(1488.10) & $31318.41(2128.66)$ & - & - & - \\
\hline & 4 & & $575.10(2269.06)$ & $30915.42(1561.21)$ & & & \\
\hline Correct & 1 & & $8.50(0.14)$ & $8.29(0.24)$ & & & \\
\hline & 2 & & $7.79(0.28)$ & $8.71(0.12)$ & 0.025 & - & 0.050 \\
\hline & 4 & & $7.71(0.50)$ & $800(0.44)$ & & & \\
\hline
\end{tabular}


Table 2. Mean scores (SE) of 10 scales of POMS.

\begin{tabular}{|c|c|c|c|c|c|}
\hline \multirow[t]{2}{*}{ Mood scales } & \multirow[t]{2}{*}{ Session } & \multicolumn{2}{|c|}{ TREATMENT } & \multicolumn{2}{|c|}{$\begin{array}{l}\text { WILCOXON SIGNED RANK } \\
\text { MDMA-Placebo }\end{array}$} \\
\hline & & MDMA & Placebo & $\mathrm{z}$ & p \\
\hline \multirow{4}{*}{ Anxiety } & 1 & $4.43(1.12)$ & $3.29(0.64)$ & -0.915 & - \\
\hline & 2 & $6.07(0.77)$ & $2.50(0.36)$ & -3.190 & 0.001 \\
\hline & 3 & $5.64(0.72)$ & $2.14(0.48)$ & -3.084 & 0.002 \\
\hline & 4 & $3.14(0.39)$ & $2.57(0.43)$ & -1.705 & - \\
\hline \multirow{4}{*}{ Depression } & 1 & $0.43(0.23)$ & $0.57(0.29)$ & -0.423 & - \\
\hline & 2 & $0.07(0.07)$ & $0.07(0.07)$ & 0.000 & - \\
\hline & 3 & $0.21(0.15)$ & $0.64(0.51)$ & -0.816 & - \\
\hline & 4 & $1.43(0.69)$ & $0.86(0.53)$ & -1.480 & - \\
\hline \multirow[t]{4}{*}{ Anger } & 1 & $1.71(0.35)$ & $2.43(0.40)$ & -1.778 & - \\
\hline & 2 & $1.50(0.36)$ & $1.29(0.29)$ & -0.734 & - \\
\hline & 3 & $1.71(0.50)$ & $1.29(0.27)$ & -1.100 & - \\
\hline & 4 & $1.57(0.56)$ & $1.07(0.27)$ & -0.777 & - \\
\hline \multirow[t]{4}{*}{ Vigor } & 1 & $15.86(0.91)$ & $15.64(1.06)$ & -0.362 & - \\
\hline & 2 & $17.57(0.96)$ & $12.29(1.24)$ & -2.766 & 0.006 \\
\hline & 3 & $17.07(1.71)$ & $10.71(1.29)$ & -2.447 & 0.014 \\
\hline & 4 & $7.00(1.50)$ & $6.21(1.25)$ & -0.567 & - \\
\hline \multirow[t]{4}{*}{ Fatigue } & 1 & $0.50(0.29)$ & $2.07(0.62)$ & -2.342 & - \\
\hline & 2 & $0.86(0.29)$ & $2.86(0.64)$ & -2.286 & - \\
\hline & 3 & $1.29(0.44)$ & $5.07(1.32)$ & -2.449 & - \\
\hline & 4 & $10.71(1.74)$ & $10.71(1.94)$ & -0.178 & - \\
\hline \multirow{4}{*}{ Confusion } & 1 & $3.79(0.51)$ & $3.00(0.31)$ & -1.848 & - \\
\hline & 2 & $6.57(0.98)$ & $3.50(0.42)$ & -2.941 & 0.003 \\
\hline & 3 & $6.00(0.84)$ & $4.14(0.48)$ & -1.695 & - \\
\hline & 4 & $5.36(0.64)$ & $5.50(0.54)$ & -0.425 & - \\
\hline \multirow[t]{4}{*}{ Friendliness } & 1 & $19.86(0.91)$ & $18.64(1.15)$ & -1.073 & - \\
\hline & 2 & $21.36(0.99)$ & $16.64(1.11)$ & -2.556 & 0.011 \\
\hline & 3 & $22.21(1.29)$ & $14.50(1.43)$ & -2.923 & 0.003 \\
\hline & 4 & $14.43(1.58)$ & $13.00(1.35)$ & -0.668 & - \\
\hline \multirow[t]{4}{*}{ Elation } & 1 & $14.07(0.72)$ & $12.57(0.77)$ & -1.740 & - \\
\hline & 2 & $16.64(0.59)$ & $10.50(0.94)$ & -3.113 & 0.002 \\
\hline & 3 & $16.64(1.20)$ & $10.00(0.99)$ & -2.959 & 0.003 \\
\hline & 4 & $8.14(1.9)$ & $7.43(1.00)$ & -0.758 & - \\
\hline \multirow{4}{*}{ Arousal } & 1 & $16.00(1.47)$ & $13.86(1.84)$ & -0.865 & - \\
\hline & 2 & $16.21(1.24)$ & $8.43(2.11)$ & -2.990 & 0.003 \\
\hline & 3 & $15.43(2.43)$ & $3.64(2.42)$ & -2.701 & 0.007 \\
\hline & 4 & $-5.93(3.45)$ & $-7.43(2.95)$ & -0.754 & - \\
\hline \multirow{4}{*}{ Positive Mood } & 1 & $13.64(0.84)$ & $12.00(0.76)$ & -1.742 & - \\
\hline & 2 & $16.57(0.56)$ & $10.43(0.98)$ & -3.113 & 0.002 \\
\hline & 3 & $16.43(1.27)$ & $9.36(1.41)$ & -2.864 & 0.004 \\
\hline & 4 & $6.71(1.72)$ & $6.57(1.41)$ & -0.039 & - \\
\hline
\end{tabular}

\section{Discussion}

Performance on different types of memory tasks (verbal, spatial) deteriorated independent of treatment, as a function of sleep deprivation. MDMA treatment produced a stable memory impairment that was consistent during the night and added to the memory impairment due to sleep deprivation. The fact that memory was impaired after administration of MDMA was in line with previous studies conducted during daytime. These acute studies showed impairment of verbal and 
spatial memory after administration of $75 \mathrm{mg}$ of MDMA (Kuypers \& Ramaekers, 2005; Kuypers \& Ramaekers, 2006).

In the spatial memory task, subjects produced larger localization errors and reacted faster after MDMA as compared with placebo. To investigate the possibility that increments in Localization Error were caused by increments in speed of responding, a regression analysis was conducted. This analysis showed that Localization Error was uncorrelated with Reaction Time and that other factors e.g. Response Delay and MDMA explained about $14 \%$ of the variance in Localization Error. This was in line with previous results that showed an increase in Localization Error when under the influence of MDMA, independent of Reaction Time (Kuypers \& Ramaekers, 2007). There was no interaction between MDMA and Time (level of sleep deprivation) on Localization Error. Localization Error increased over successive sessions when hours of sleep loss increased in both MDMA and placebo condition. Reaction time however showed a U-curve shaped progression over sessions. Reaction time decreased during the evening and night, in session 2 and 3, after administration of the drug, whereas during the morning session it increased to placebo-like levels. Apparently, there is a discrepancy between the stimulant-like effects of MDMA on motor behavior which seem to have worn off during the morning, and the impairing effects it has on memory performance, which were still present in the morning. The presence of the memory impairment in the morning was in line with the blood plasma concentrations which at that point were comparable with those measured $1.5 \mathrm{~h}$ after administration of $75 \mathrm{mg}$ of MDMA. This discrepancy between effects of MDMA on memory performance and psychomotor performance was, besides the regression analysis, additional support for the view that the impairment of memory after MDMA administration was not due to increments in response speed.

Performance on the verbal memory task, i.e. the Sternberg memory scanning task, deteriorated over time and independent of treatment. The speed of retrieval of verbal stimuli from short-term memory slowed down during the night and in the morning. MDMA added to this impairment and caused a larger speed reduction of retrieval speed compared to placebo. There was no interaction between MDMA and Time on the speed of retrieval. This indicated that the rates of decline in speed of retrieval as a function of hours of sleep loss were similar after MDMA and placebo. Subjects' performance on the working memory task, i.e. the star counting task, was compromised by MDMA, especially during the nighttime. However, in the morning when the sleep deprivation was maximal, performance was comparable to placebo. Performance in the placebo condition 
showed a clear decrement over time, with the worst performance in the morning, when the level of sleep deprivation was maximal. This demonstrates that the impairing effect of MDMA on working memory performance was no longer present during the morning session, when subjects' performance after repeated MDMA doses was comparable to that after placebo.

There was a clear interaction between the MDMA effect on mood and hours of sleep loss. After administration of MDMA, feelings of vigor, friendliness, elation, anxiety, confusion, arousal and positive mood seemed to increase in magnitude during the night, while they returned to placebo-like levels on the final morning session. MDMA did not affect measures of depression, fatigue and anger. The interaction between MDMA's effects on mood and hours of sleep loss seemed to be in line with the stimulant effects MDMA had on reaction time performance in the spatial memory task. This stimulant effect on reaction time also disappeared during the morning session when sleep loss was maximal even though MDMA concentrations in blood which were in the morning as high as after the first dose given in the evening. This discrepancy between kinetics and dynamics is also known as hysteresis, which means that the pharmacological effect is dependent on the time since dose as well as on the concentration in the blood (Logan \& Isenschmid, 2003). The time since dose in the morning, in the present study, was 11 and 7 hours for the $1^{\text {st }}$ and $2^{\text {nd }}$ dose respectively. This means that subjects were at that moment in the elimination phase, as the half-life of MDMA is approximately 8 hours (de la Torre et al., 2004). Its stimulating effects disappeared or were not high enough to compensate for the sleep loss as demonstrated by the interaction effect on reaction time in the spatial memory task and several scales of the POMS.

Previous acute studies have shown that a single dose of MDMA impaired verbal and spatial memory and improved response speed. The present study showed that after a single and repeated dose of MDMA, memory was impaired and that this added to the memory impairment due to sleep deprivation. During the nighttime sessions, MDMA exerted a stimulant-like effect on response speed which was also previously observed during day-time testing. This stimulant effect disappeared in the morning session, after a night of sleep deprivation. The same pattern was also reflected in ratings of mood, which were elevated after acute dosing of MDMA but decreased in the morning, when sleep deprivation was maximal. 
70 | CHAPTER 4

It is concluded that evening doses of MDMA selectively impair memory performance, and that this impairment is additional to the effect of sleep deprivation on memory performance.

\section{Acknowledgments}

We would like to thank. Nele Samyn, Gert De Boeck and Maria del Mar Ramirez Fernandez. from NICC, Brussels, for analyzing MDMA blood plasma samples and Nina Limbert for her belp with the data collection. 


\section{References}

Cami, J., Farre, M., Mas, M., Roset, P. N., Poudevida, S., Mas, A., et al. (2000). Human pharmacology of 3,4-methylenedioxymethamphetamine ("ecstasy"): psychomotor performance and subjective effects. Journal of clinical psychopharmacology, 20(4), 455466.

Cole, J. C., \& Sumnall, H. R. (2003). Altered states: the clinical effects of Ecstasy. Pharmacology and Therapeutics, 98, 35-58.

de Jong, P. F., \& Das Smaal, E. A. (1995). Attention and intelligence: The validity of the Star Counting Test. Journal of Educational Psychology, 87(1), 80-92.

de la Torre, R., Farre, M., Ortuno, J., Mas, M., Brenneisen, R., Roset, P. N., et al. (2000). Non-linear pharmacokinetics of MDMA ('ecstasy') in humans. British journal of clinical pharmacology, 49(2), 104-109.

de la Torre, R., Farre, M., Roset, P. N., Pizarro, N., Abanades, S., Segura, M., et al. (2004). Human pharmacology of MDMA: pharmacokinetics, metabolism, and disposition. Therapeutic drug monitoring, 26(2), 137-144.

de Wit, H., Enggasser, J. L., \& Richards, J. B. (2002). Acute administration of damphetamine decreases impulsivity in healthy volunteers. Neuropsychopharmacology official publication of the American College of Neuropsychopharmacology, 27(5), 813-825.

DIMS. (2005). DIMS Jaarbericht 2005: Trimbos institute Netherlands Institute of Mental health and Addiction.

Farre, M., De La Torre, R., O Mathuna, B., Roset, P. N., Peiro, A. M., Torrens, M., et al. (2004). Repeated doses administration of MDMA in humans: pharmacological effects and pharmacokinetics. Psychopharmacology, 173(3-4), 364-375.

Gouzoulis Mayfrank, E., \& Daumann, J. (2006). The confounding problem of polydrug use in recreational ecstasy/MDMA users: A brief overview.

Hammersley, R., Ditton, J., Smith, I., Short, E. (1999). Patterns of ecstasy use by drug users. British journal of criminology, 39(4), 625-647.

Kuypers, K. P. C., \& Ramaekers, J. G. (2005). Transient memory impairment after acute dose of 75mg 3.4-Methylene-dioxymethamphetamine. Journal of psychopharmacology Oxford, England, 19(6), 633-639.

Kuypers, K. P. C., \& Ramaekers, J. G. (2007). Acute dose of MDMA (75 mg) impairs spatial memory for location but leaves contextual processing of visuospatial information unaffected. Psychopharmacology, 198(4), 557-563. 
Logan, B. K., \& Isenschmid, D. S. (2003). Cocaine, methamphetamine and driving impairment. In M. Burns (Ed.), Medical-legal aspects of drugs (first edition ed., Vol. Chapter 8, pp. 215-234). Tucson, US: Lawyers and Judges publishing company.

McEwen, B. S. (2006). Sleep deprivation as a neurobiologic and physiologic stressor: Allostasis and allostatic load. Metabolism clinical and experimental, 55(10 Suppl 2), S20-23.

Mulder-Hajonides van der Meulen, W. R. E. H., Wijnberg, J. R., Hollanders, J. J., DeDiana, I., \& Hoofdakker, R. (1980). Measurement of subjective sleep quality. Paper presented at the Fifth European Congress on Sleep Research, Amsterdam.

Parrott, A. C. (2001). Human psychopharmacology of Ecstasy (MDMA): A review of 15 years of empirical research. Human Psychopharmacology Clinical and Experimental, 16(8), 557-577.

Parrott, A. C. (2006). MDMA in humans: Factors which affect the neuropsychobiological profiles of recreational ecstasy users, the integrative role of bioenergetic stress.

Parrott, A. C., \& Lasky, J. (1998). Ecstasy (MDMA) effects upon mood and cognition: before, during and after a Saturday night dance. Psychopharmacology, 139(3), 261268.

Sternberg, S. (1966). High-speed scanning in human memory.

Thomasius, R., Zapletalova, P., Petersen, K., Buchert, R., Andresen, B., Wartberg, L., et al. (2006). Mood, cognition and serotonin transporter availability in current and former ecstasy (MDMA) users: The longitudinal perspective.

Vermeeren, A., Jackson, J. L., Muntjewerff, N. D., Quint, P. J., Harrison, E. M., \& O'Hanlon, J. F. (1995). Comparison of acute alprazolam (0.25, 0.50 and $1.0 \mathrm{mg})$ effects versus those of lorazepam $2 \mathrm{mg}$ and placebo on memory in healthy volunteers using laboratory and telephone tests. Psychopharmacology, 118(1), 1-9. 


\title{
CHAPTER 5
}

\section{IMAGING THE ACUTE EFFECTS OF A SINGLE DOSE OF MDMA ON WORD LEARNING -PRELIMINARY RESULTS-}

\author{
Kuypers, K.P.C., Wingen, M., Formisano, E., and Ramaekers, J.G.
}

Background. Previous research has shown that a single dose of MDMA causes transient impairment of verbal memory. Objective. The aim of the present study was to investigate the neural substrates underlying MDMA-induced memory impairment. Methods. Seven healthy male recreational MDMA users participated in the study. The study was conducted according to a double-blind, placebocontrolled within-subject design. Treatments were $75 \mathrm{mg}$ of MDMA and placebo. Prior to drug-intake, subjects had to memorize a list of 15 words (control list). One and a half hours after drug administration, they were placed in a 3-Telsa scanner. In the scanner, they were presented two word lists each in alternation. One list was the control list and the other the experimental list, containing 15 new words. Subjects' task was to read the words quietly and learn them. Thirty-second blocks of the experimental list were alternated with 30 -second blocks of the control list and this procedure was repeated five times. After each list-presentation, subjects had to recall as much words as possible. Behavioral data included the number of words correctly recalled on each trial, and their sum over the 5 trials. Functional data consisted of recordings of the BOLD signal during presentation of both lists. Results. Word learning was mainly related with activation patterns in frontal regions. Under influence of MDMA, the fMRI response increased independent of list-type in the left middle frontal area which is involved in word learning. On the behavioural level, memory performance tended to be lower under the influence of MDMA, but failed to reach statistical significance. Discussion. It was shown that a single dose of MDMA caused an increase in BOLD activity in the experimental as well as the control list condition. This seems to indicate that the left middle frontal area which is involved in word learning underlies memory impairment observed at the behavioral level. The preliminary conclusion is that MDMA affects verbal memory functioning by interfering with the word learning or encoding phase. 
74 | CHAPTER 5 


\section{Introduction}

Previously, a causal association between pharmacological exposure of MDMA and memory impairment was established in an acute, placebo-controlled study. It was shown that immediate and delayed recall of words was impaired after a single dose of MDMA. Although subjects under the influence of MDMA showed a normal learning curve, they learnt less as compared with placebo (Kuypers \& Ramaekers, 2005). Based on these results it was hypothesized that this deficit could perhaps be attributed to a specific influence of MDMA on encoding of words.

Imaging studies have implicated frontal and temporal areas in the encoding of verbal material, and more specifically posterior and more ventral regions of the left frontal cortex and medial temporal lobe structures (Buckner, Kelley, \& Petersen, 1999; Dupont, Samson, Le Bihan, \& Baulac, 2002; Gabrieli, Poldrack, \& Desmond, 1998; Ojemann, Buckner, Corbetta, \& Raichle, 1997; Schacter \& Wagner, 1999; Smith, Jonides, Marshuetz, \& Koeppe, 1998). Frontal areas supply information to medial temporal lobe structures, and these structures are subsequently implied in the formation of memory (Buckner et al., 1999; Squire \& Zola Morgan, 1991).

The aim of the present study was to identify the neural substrate underlying memory impairment due to a single dose of MDMA. The present study was conducted according to a double-blind, placebo-controlled, within-subject design. Treatments were MDMA (75 mg) and placebo. The experimental task was hypothesized to involve word learning/ encoding and reading processes whereas the control task was hypothesized to involve only reading. Subtraction of the control images from the experimental task images should reveal activity related to word-learning. It was hypothesized that frontal and temporal areas, often linked with memory encoding would be especially active during the encoding or wordlearning phase and second that MDMA would affect these encoding related areas.

The data presented in this chapter were part of a larger fMRI study investigating the acute effects of a single dose of MDMA on verbal memory and prospective memory performance. The study, which is presently ongoing, is aiming at a sample size of $\mathrm{N}=12$. The results presented here should be considered as preliminary. 


\section{Methods}

\section{Design and treatments}

The study was conducted according to a double-blind, placebo controlled, randomized, crossover design with balancing of the treatments. The memory task was conducted between 1.5 and 2 hours post-drug. The treatments were a single dose of MDMA (75mg) and placebo. Placebo and MDMA were administered orally, in identically appearing formulations. MDMA was administered as a $25 \mathrm{ml}$ solution in bitter orange peel syrup and mixed with $200 \mathrm{ml}$ of orange juice.

\section{Participants}

Participants were seven healthy male recreational ecstasy users (6 right-handed; one left-handed), aged in the range of 20 years to 29 years (mean age (SD): 24.86(3.24)). Lifetime use of MDMA varied from light ( $<18$ times) in 5 subjects to heavy in two subjects (35 and 200 times). They were all native Dutch speakers.

Subjects were recruited by means of advertisements in local newspapers and by word of mouth. Potential candidates were questioned about their drug use and medical condition and were given information about the study. Thereupon they were sent a detailed brochure with information about the study procedure, two questionnaires for medical history and detailed history of drug use, and an fMRI screenings list. Inclusion criteria were experience with MDMA use, free from psychotropic medication, good physical health, absence of major medical endocrine and neurological conditions, normal weight (Body Mass Index 18-28 $\mathrm{kg} / \mathrm{m}^{2}$ ). Exclusion criteria were history of drug abuse (other than MDMA) or addiction, pregnancy or lactation, cardiovascular abnormalities (assessed by a standard 12-lead electrocardiogram (ECG)), excessive drinking ( $>20$ alcoholic consumptions per week), hypertension (diastolic $>100 \mathrm{mmHg}$; systolic $>170$ $\mathrm{mmHg}$ ), history of psychiatric or neurological disorders, presence of parts with magnetic properties in the body (fMRI criterion). A medical doctor checked the completed medical questionnaire, and upon approval, subjects were invited for a medical examination. Blood and urine samples were taken for examination and they underwent an ECG measurement. If medically fit, they were contacted and sent a brochure with information about the study and a number of rules they had to obey during the period of the study. Subjects signed an informed consent. They were paid for participating upon completion of the study.

The study was performed in accordance with the 1975 declaration of Helsinki, adjusted in Edinburgh (2000) and was approved by the Medical Ethics Committee 
of the Academic Hospital of Maastricht and the University of Maastricht. A permit for obtaining, storing and administering MDMA was obtained from the Dutch drug enforcement administration.

\section{Study procedure}

Before study onset, subjects were familiarized with the task in a short training session and they were given a list of 15 monosyllabic words which they had to learn by heart. This list then served as control list on both test days.

On the test day, subjects were screened for drugs and alcohol in their urine and breath, respectively, upon arrival at the test facilities at $8.30 \mathrm{AM}$. If negative, subjects proceeded with a light breakfast. Subjects filled out a sleep questionnaire and after breakfast it was checked whether they could perfectly recall the fifteen words of the control list. Half an hour later ( 9 AM) subjects received a drink containing either placebo or MDMA. Seventy-five minutes after drug-intake, subjects had to fill out a mood questionnaire, and a blood sample was taken to determine MDMA/MDA concentrations in blood serum afterwards (this quantification will be conducted after study completion and is not presented in this chapter). Following this, subjects were accompanied to the MR-scanner and placed on the scanner bed and provided with earplugs to reduce the noise and a head phone set, for communication with the person operating the scanner. The subjects' head was fixed by means of foam pads to restrict head motion. The tasks were projected onto a screen at the end of the scanner bore, and viewed via a mirror mounted on the volume head-coil. Headphones and earplugs were provided. The verbal word learning task started approximately 1.5 hours post-drug, followed by another memory task of 20 minutes (not reported on in this chapter) and finally the anatomical scan. When the scanning finished, subjects were asked outside the scanner to recall as much words, of both lists, they remembered.

An additional blood sample was taken every $7^{\text {th }}$ day (at $2^{\text {nd }}$ test day) and $11^{\text {th }}$ day (after $2^{\text {nd }}$ test day) to control for the integrity of renal and liver functioning. The minimum washout period between sessions was 7 days.

\section{Cognitive assessment}

Word-learning task. The word-learning task used in the present study is an adjusted version of the one Kuypers and Ramaekers (2005) used in a previous behavioural study (Kuypers \& Ramaekers, 2005; Rey, 1958). The task was customised to suit blocked imaging, which was used to examine brain regions specialized for learning and/or reading words. 
The word-learning test began with memorizing the experimental list, a list of 15 monosyllabic concrete nouns. Each word was shown on the computer display for 1 second and the subject read it silently. When the series ended, a 10second blank was presented, followed by an instruction screen, telling the subjects to start to recall as many words as possible within a time frame of 60 seconds.. Thereupon the control list, which was also a list of 15 monosyllabic words, learned before the session and drug administration, was shown in the same manner as the experimental list. At the end of the control list subjects also had to recall as many words as possible. The two lists were presented in alternation on five successive occasions. A stars count-down preceded each list to signal the beginning of the list for the subject. During scanning, speech was recorded (Goldwave version 4.26) and processed off-line by means of a scanner noise reduction program (Cusack, Cumming, Bor, Norris, \& Lyzenga, 2005). After the reduction of the scanner noise, it was possible to hear and list the words subjects recalled during the recall periods. The numbers of words correctly recalled in the five experimental trials were summed to yield the total immediate free recall score, a measure of learning or working memory.

After a 40 minutes delay, during which subjects performed a second memory task (not reported here), subjects were asked (outside the scanner) to recall as many words as possible. The number of words correctly recalled was taken as the delayed recall score.

The difference between the control list and the experimental list was that during the presentation of the experimental list, subjects had to read and learn these words and only read the words of the control list because these had been memorized already.

\section{Subjective assessment}

Two subjective measurements were taken: i.e. the Groninger Sleep Scale (GSS) and the Profile of Mood States (POMS). The GSS assessed sleep quality and quantity (hours of sleep). It consists of fifteen dichotomous questions about sleep complaints and an open question concerning the duration of sleep the previous night (Mulder-Hajonides van der Meulen, Wijnberg, Hollanders, DeDiana, \& Hoofdakker, 1980). The POMS (de Wit, Enggasser, \& Richards, 2002) is a selfassessment mood questionnaire with 72 five-point Likert scale items, representing eight mood states; i.e. Anxiety, Depression, Anger, Vigor, Fatigue, Confusion, Friendliness and Elation. Two extra scales were derived, i.e. Arousal ((Anxiety + Vigor $)-($ Fatigue + Confusion) $)$ and Positive mood (Elation - Depression). The subject had to indicate to what extent these items were representing his/her mood. 


\section{Behavioural and subjective data analysis}

All data was analysed using the statistical package SPSS version 11.5. The alpha criterion level of significance was set at $p=0.05$. Data were analysed with the nonparametric Wilcoxon signed ranks test.

\section{Image acquisition}

Imaging was conducted in a 3-Tesla Siemens Allegra MR head-only scanner, equipped with a standard head coil and echo planar sequences for ultra-fast MRimaging (Allegra, Siemens medical systems). Anatomical T1-weighted images were acquired using a 3-D modified driven equilibrium Fourier transform (MDEFT) sequence with an isotropic spatial resolution of $1 \mathrm{~mm}$. An anatomical run contained 176 slices, lasted 12 minutes, and was performed for each subject in each condition.

Blood oxygen level dependent (BOLD) fMRI images were acquired with a gradient echo $\mathrm{T} 2 *$-weighted image sequence with the following parameters: $\mathrm{TR}=$ $2000 \mathrm{~ms}$; TE $=30 \mathrm{~ms}$; voxel size $=3.5 \times 3.5 \times 3.5 \mathrm{~mm}$; no gap between slices, interleaved slice sampling; flip angle $=90$ degrees; matrix size $=64 * 64$. Functional time series consisted of 32 slices and 600 volumes, and lasted 20 minutes. During this time, the experimental list was shown 5 times in alternation with the control list. Each list presentation was followed by a recall period; (15 scans per list presentation).

\section{fMRI data analysis}

Data analysis, registration and visualization were performed with the fMRI software package Brain Voyager QX version 1.6 (www.brainvoyager.com). Before statistical analysis, functional data were preprocessed (3D motion correction (trilinear interpolation, reduced data), slice scan time correction (sinc interpolation, ascending and interleaved slice scanning order), and temporal data filtering (linear trend removal, high pass filter of 3 cycles/run). The estimates of the six motion parameters from this analysis were included, together with the 12 predictors (experimental list 1-5, control list 1-5, recall, and stars) from the task, in the multisubject GLM analysis.

Whole brain fixed effects analysis with three contrasts was performed on the imaging data. The task effect was computed by means of a contrast of the experimental list minus the control list, in the placebo condition. The drug effect on activation was computed by means of a contrast of MDMA minus placebo, independent of list-type. The third contrast included the interaction of treatment 
(MDMA/Placebo) by task (encoding \& reading/reading). The threshold was set at pFDR $<0.001$ (False Discovery Rate correction) and the cluster size threshold at 100.

In regions showing significant activation, epoch-based event-related averaging was conducted to detect whether selected conditions (e.g. experimental list/ MDMA) showed differences in bold response compared with a reference condition (control list/ placebo). To investigate the drug by task interaction, only areas which showed a significant task effect were inspected for drug effects.

\section{Results}

\section{Missing data}

One behavioural data set was missing for a subject in the MDMA condition. The behavioural data set therefore contains only 6 subjects. Complete datasets $(N=7)$ were collected for imaging and mood parameters.

\section{Cognitive assessment (Word-learning task)}

Drug effect. There were no significant effects of MDMA on number of correct per trial, and in total, nor on delayed recall, of the experimental and control list (Table 1, Fig.1). Task effect. Analysis revealed that the experimental and the control list differed significantly from each other on total correct (immediate recall) ( $\mathrm{z}=$ 2.201; $\mathrm{p}=0.028)$ and on delayed recall $(\mathrm{z}=-2.023 ; \mathrm{p}=0.043)$.

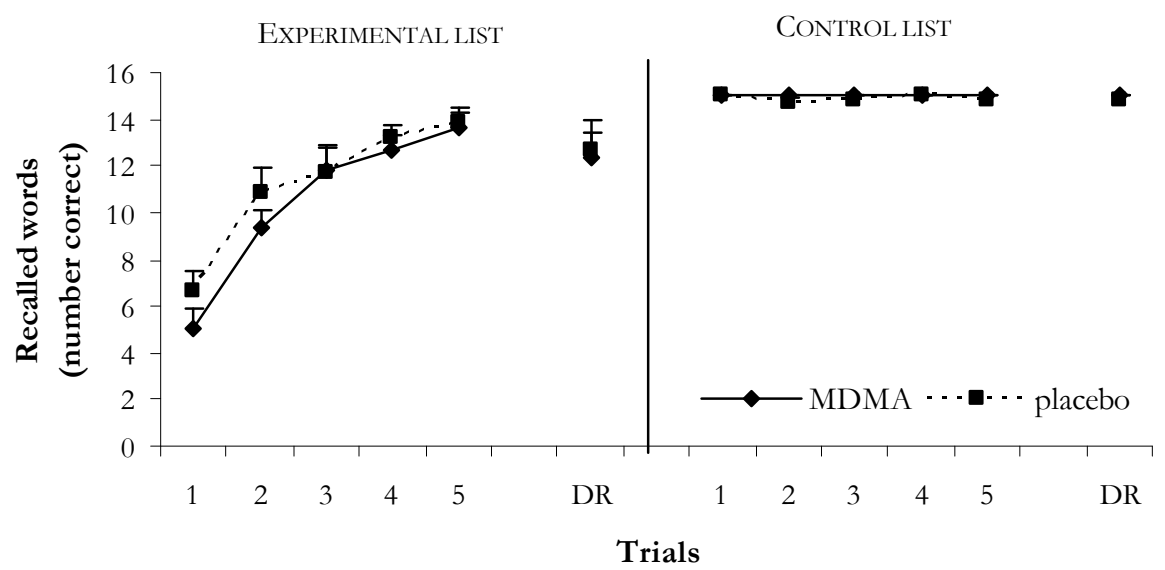

Figure 1. In this figure the number of correct recalled words per trial in the control list and the experimental list for both the MDMA and the placebo condition is shown. 
Table 1. Mean scores (SE) of correct recalled words per trial in the control list and the experimental list for both the MDMA and the placebo condition.

\begin{tabular}{lcccc} 
& \multicolumn{2}{c}{ TREATMENT } & \multicolumn{2}{c}{ WILCOXON SigNED RANK } \\
& MDMA & Placebo & $\mathrm{z}$ & $\mathrm{p}$ \\
EXPERIMENTAL LIST & & & & .057 \\
IR (Trial 1) & $5(.93)$ & $6.67(.88)$ & -1.90 & - \\
IR (Trial 2) & $9.33(.76)$ & $10.83(1.14)$ & -1.48 & - \\
IR (Trial 3) & $11.83(.95)$ & $11.67(1.26)$ & -.14 & - \\
IR (Trial 4) & $12.67(.67)$ & $13.17(.60)$ & -1.73 & - \\
IR (Trial 5) & $13.67(.61)$ & $13.83(.65)$ & -.58 & - \\
IR (Total) & $52.50(3.11)$ & $56.17(4.06)$ & -1.47 & - \\
DR & $12.33(1.05)$ & $12.67(1.33)$ & -.53 & - \\
CONTROL LIST & & & & - \\
IR (Trial 1) & $15(.00)$ & $15(.00)$ & .00 & - \\
IR (Trial 2) & $15(.00)$ & $14.67(.21)$ & -1.41 & - \\
IR (Trial 3) & $15(.00)$ & $14.83(.17)$ & -1.00 & - \\
IR (Trial 4) & $15(.00)$ & $15(.00)$ & .00 & - \\
IR (Trial 5) & $15(.00)$ & $14.83(.17)$ & -1.00 & - \\
IR (Total) & $75.00)$ & $74.33(.33)$ & -1.63 & - \\
DR & $15(.00)$ & $14.83(.17)$ & -1.00 &
\end{tabular}

Table2. Mean scores (SE) of 10 scales of POMS.

\begin{tabular}{lcccc} 
& \multicolumn{2}{c}{ TREATMENT } & \multicolumn{2}{c}{ WilCOXON SiGNED RANK } \\
MDMA-Placebo \\
POMS SCALES & MDMA & PLACEBO & $\mathrm{Z}$ & $\mathrm{P}$ \\
Anxiety & $6.28(3.15)$ & $2.43(1.40)$ & -1.997 & .046 \\
Depression & $.28(.49)$ & $.43(.79)$ & -.577 & - \\
Anger & $.71(1.11)$ & $1.43(1.62)$ & -1.069 & - \\
Vigor & $16.57(6.11)$ & $13.43(6.97)$ & -1.892 & .058 \\
Fatigue & $1.28(1.25)$ & $1.00(1.91)$ & -.541 & - \\
Confusion & $7.86(2.91)$ & $2.57(2.07)$ & -2.371 & .018 \\
Friendliness & $18.28(7.54)$ & $16.86(7.49)$ & -.843 & - \\
Elation & $12.57(3.82)$ & $10.28(3.82)$ & -2.032 & .042 \\
Arousal & $13.71(9.21)$ & $12.28(8.30)$ & -.509 & - \\
Positive Mood & $12.28(3.68)$ & $9.86(3.89)$ & -2.232 & .026
\end{tabular}

\section{Functional imaging data}

A summary of drug effect, task effect and drug by task effects is given in table 3 .

Main effect of task. The verbal encoding activity was examined by means of the first contrast, comparing experimental list-activation with control list- activation, in the placebo condition. Mainly frontal and parietal areas showed a bilateral activation pattern. After event-related averaging in these activated regions, six regions (i.e. right and left medial frontal area, left frontal operculum (inferior frontal gyrus), left putamen, right medial cingulum and right supplemental motor area) revealed a larger bold signal during the experimental list compared with the control list (See table 3). An example of fMRI responses on the experimental versus the control list in the left medial frontal region is shown in figure 2. 

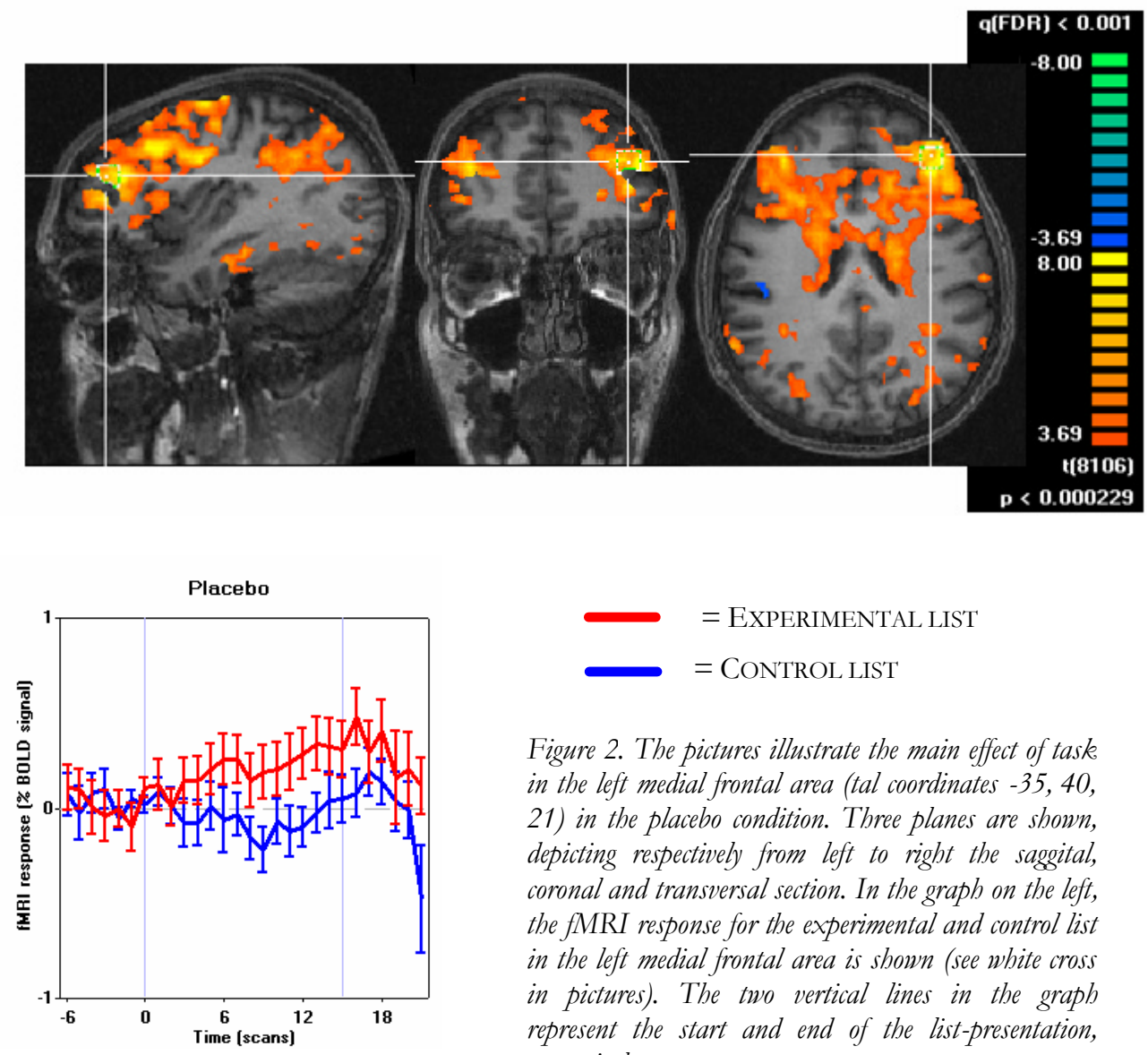

Figure 2. The pictures illustrate the main effect of task in the left medial frontal area (tal coordinates -35, 40, 21) in the placebo condition. Three planes are shown, depicting respectively from left to right the saggital, coronal and transversal section. In the graph on the left, the fMRI response for the experimental and control list in the left medial frontal area is shown (see white cross in pictures). The two vertical lines in the graph represent the start and end of the list-presentation, respectively.

Main effect of drug. The main effect of drug on activity was computed comparing the MDMA-related activation with the placebo-related activation, in both the experimental and control list. A number of cortical regions (parietal and temporal) demonstrated primarily left lateralised activity except for the occipital lobe, which was bilaterally activated. After event-related averaging in these activated regions, a larger MDMA-related fMRI response was observed compared with placebo in almost all significantly activated regions i.e. left and right inferior occipital gyrus, left medial occipital gyrus, left medial and inferior temporal gyrus, left inferior parietal gyrus, right lingual gyrus, and left precuneus (Table 3). An example of fMRI responses in the MDMA versus the placebo condition in the occipital lobe is shown in figure 3. 

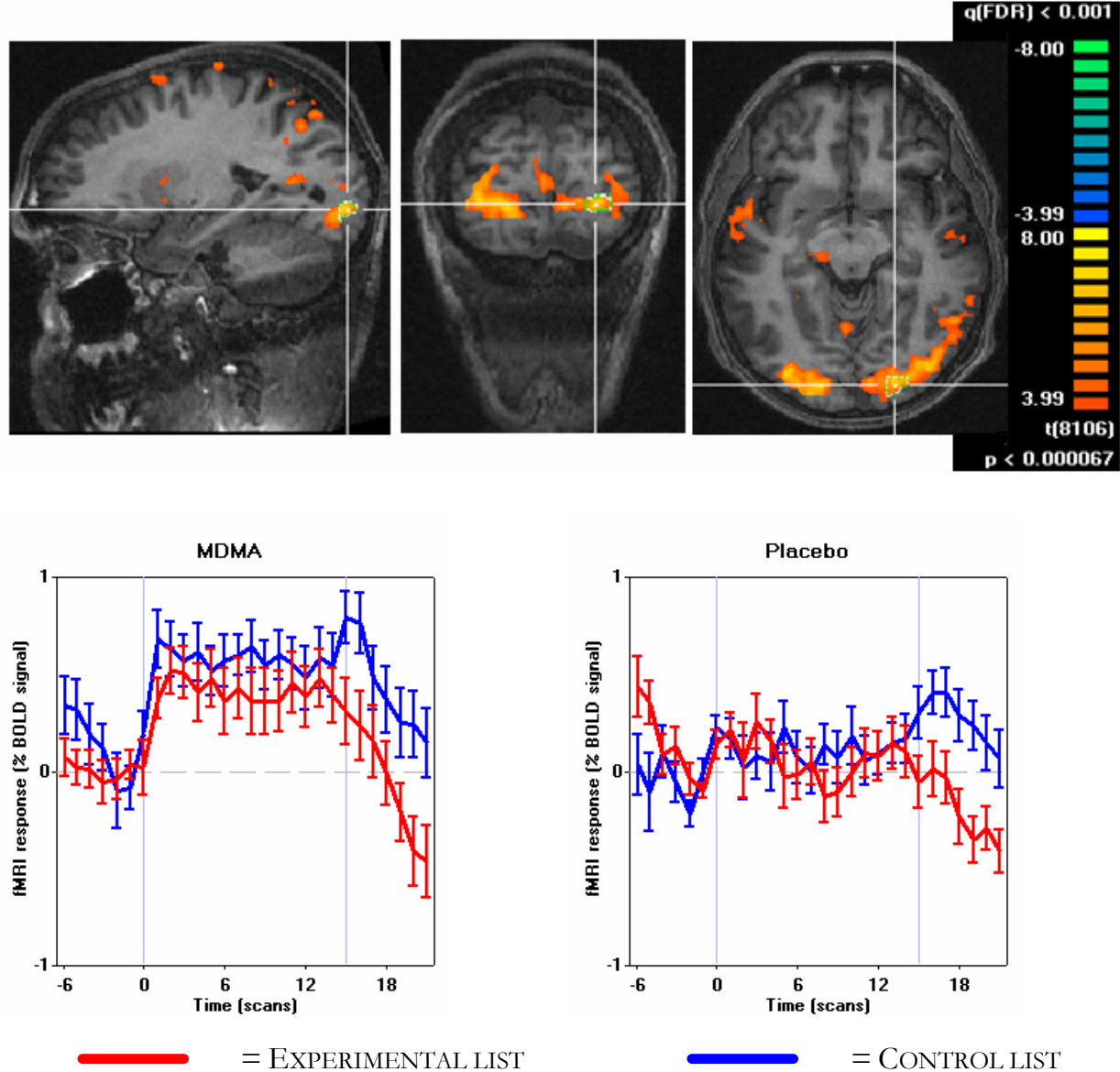

Figure 3. The top pictures illustrate the main effect of drug in the left inferior occipital area (tal coordinates 22, -87, -7) as computed by the main drug effect contrast. Three planes are shown, depicting respectively from left to right the saggital, coronal and transversal section. In the graphs underneath, the fMRI response in the $M D M A$ condition (left graph) and the placebo condition (right graph) for both lists in the left inferior occipital area is shown (see white cross in pictures). The two vertical lines in the graphs represent the start and end of the list-presentation, respectively.

Interaction effect of drug by task. The drug by task interaction effect was computed by subtracting the task effect in the placebo condition from the task effect in the MDMA condition. This analysis revealed mainly decreased activation in a limited number of regions (e.g. left medial frontal gyrus, left medial temporal gyrus and left hippocampus). Only regions which showed a significant task-effect (see table 3) were inspected. This resulted in one area i.e. left medial frontal gyrus which was subjected to event-related averaging. The fMRI response was larger in the MDMA condition compared with the placebo condition, during the control task in the left medial frontal gyrus (see figure 4). 

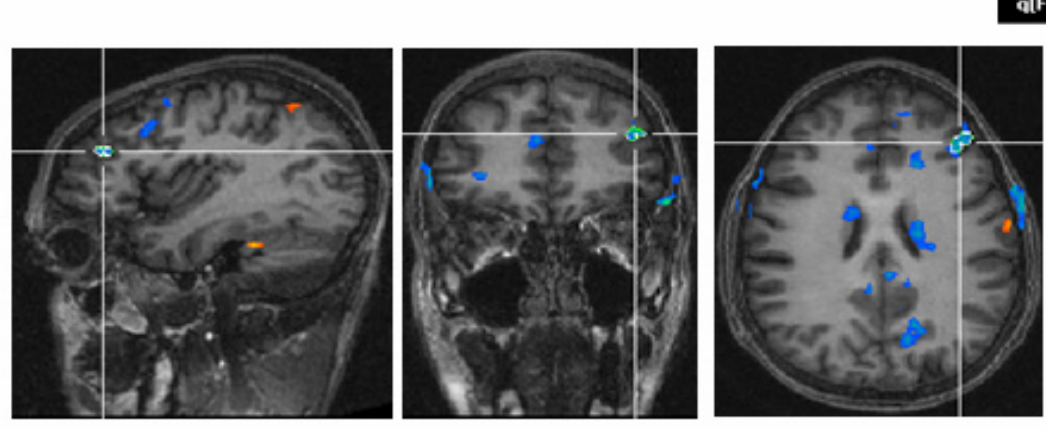

a(FDR $<0.001$
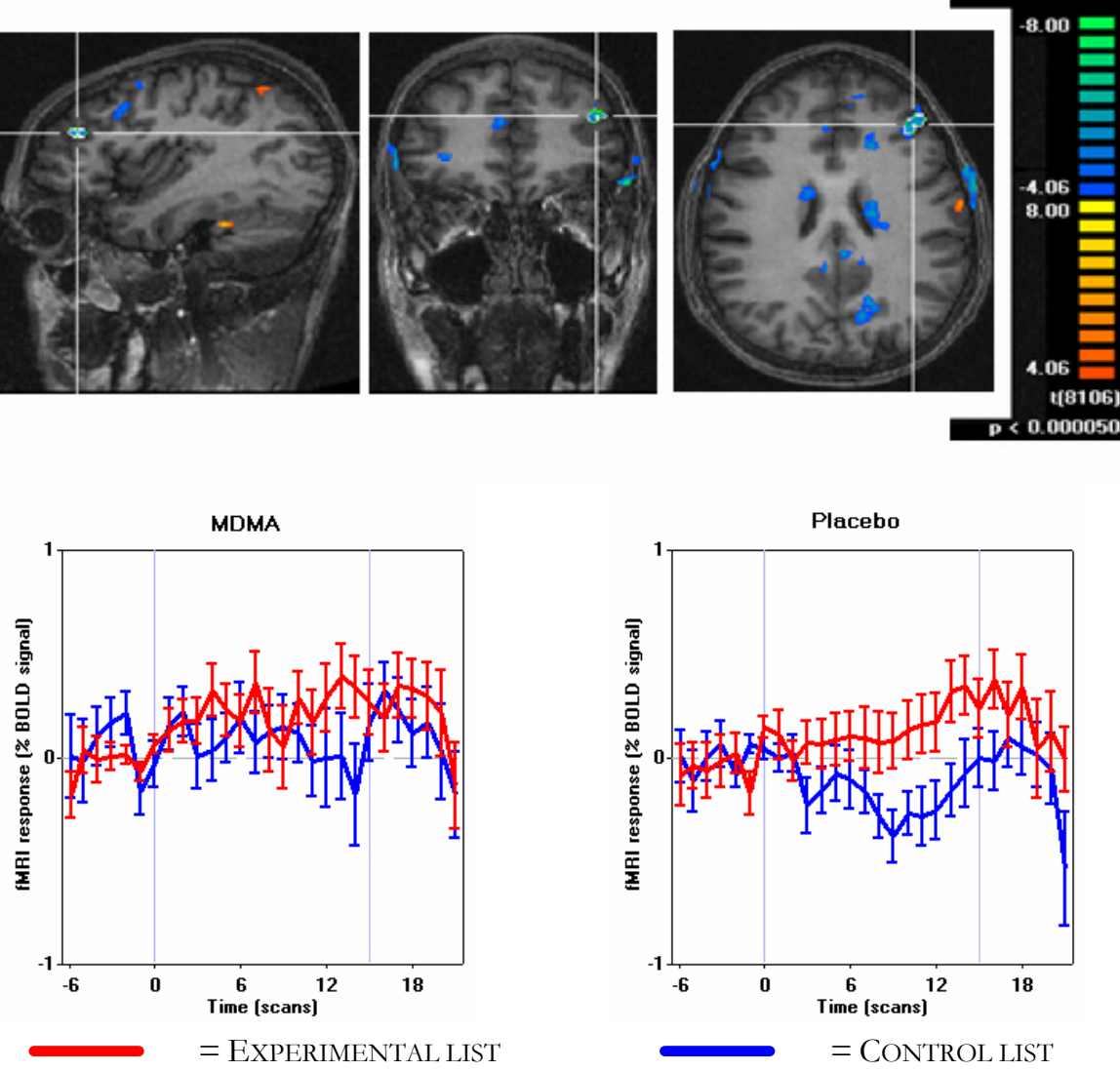

Figure 4. The top pictures illustrate the drug by task interaction in the left medial frontal area (tal coordinates $-37,35,22)$ as computed by the interaction'-contrast. Three planes are shown, depicting respectively from left to right the saggital, coronal and transversal section. In the graphs underneath, the $f M R I$ response in the MDMA condition (left graph) and the placebo condition (right graph) for both lists in the left medial frontal gyrus is shown (see white cross in pictures). The two vertical lines in the graphs represent the start and end of the list-presentation, respectively.

Table 3. Talairach $(x, y, z)$ coordinates and t-values and number of voxels for regions showing activation in the three contrasts (showing main activation of task, drug or drug by task) increased and decreased activation are indicated by a plus-sign and minus-sign, respectively. All reported t-values were significant at $q=0.001$.

\begin{tabular}{|c|c|c|c|c|c|c|c|c|}
\hline \multirow{2}{*}{\multicolumn{2}{|c|}{ Region of activation }} & \multirow{2}{*}{$\begin{array}{l}\text { Left/ } \\
\text { Right }\end{array}$} & \multirow{2}{*}{ Activation } & \multicolumn{3}{|c|}{ Tailarach coordinates } & \multirow{2}{*}{ t-value } & \multirow{2}{*}{$\begin{array}{c}\# \\
\text { voxels }\end{array}$} \\
\hline & & & & $x$ & $y$ & $z$ & & \\
\hline \multicolumn{9}{|c|}{-TASK EFFECT-PLACEBO (EXPERIMENTAL LIST MINUS CONTROL LIST) } \\
\hline \multirow[t]{2}{*}{ Frontal } & Medial & $\begin{array}{l}\mathrm{L} \\
\mathrm{R}\end{array}$ & $\begin{array}{l}+ \\
+\end{array}$ & $\begin{array}{r}-35 \\
39\end{array}$ & $\begin{array}{l}40 \\
29\end{array}$ & $\begin{array}{l}21 \\
32\end{array}$ & $\begin{array}{l}6.51 \\
5.10\end{array}$ & $\begin{array}{l}1141 \\
1271\end{array}$ \\
\hline & $\begin{array}{l}\text { Inferior } \\
\text { Operculum }\end{array}$ & $\mathrm{L}$ & + & -39 & 16 & 34 & 7.37 & 1315 \\
\hline $\begin{array}{l}\text { Putamen } \\
\text { Cingulum } \\
\text { SMA }\end{array}$ & Medial & $\begin{array}{l}\mathrm{L} \\
\mathrm{R} \\
\mathrm{R}\end{array}$ & $\begin{array}{l}+ \\
+ \\
+\end{array}$ & $\begin{array}{c}-26 \\
6 \\
1\end{array}$ & $\begin{array}{l}10 \\
12 \\
13\end{array}$ & $\begin{array}{c}3 \\
43 \\
47\end{array}$ & $\begin{array}{l}4.55 \\
5.58 \\
5.53\end{array}$ & $\begin{array}{c}847 \\
985 \\
1047\end{array}$ \\
\hline
\end{tabular}


Table 3. Continued

\begin{tabular}{llccccccc} 
Region of activation & $\begin{array}{l}\text { Left/ } \\
\text { Right }\end{array}$ & Activation & \multicolumn{2}{c}{ Tailarach coordinates } & t-value & \# \\
voxels
\end{tabular}

\section{Discussion}

A single dose of MDMA caused a selective increase in activation in regions related with word learning i.e. left medial frontal gyrus, irrespective of list-type. On the behavioral level, no significant effects of MDMA on immediate recall, an indirect measure of word learning, were found. However, there was a tendency for memory performance in the MDMA condition to be inferior to memory performance in the placebo condition. Under influence of MDMA, subjects recalled 4 words less in total compared with the number of words recalled during the placebo condition. In a previous study by Kuypers and Ramaekers (2005), a similar effect achieved significance in 18 subjects (Kuypers \& Ramaekers, 2005). Given the small sample size $(n=6)$ of the present data set it is to be expected that memory impairment will become apparent when the statistical power is increased by means of a larger sample size.

To determine the main effect of MDMA on brain activation patterns, MDMA-related activation was compared with placebo-related activation, independent of list-type. It was shown that there was an increase in activity in a number of regions that were not selectively activated by a specific list, while subjects were under influence of MDMA, compared with placebo. An example is the pronounced bilateral activation pattern in the occipital areas. Activation in this region was also demonstrated by Brevard and colleagues (2006) when imaging brain activity in monkeys after administration of a single dose of MDMA. It was shown that especially occipital areas were activated more in presence of visual stimuli compared with a baseline (off-drug) condition. 
To determine the task effect on brain activation patterns, experimental list activation was compared with control list activation in the placebo condition. A region which was significantly involved in word learning in the present paradigm was the cingulum. This structure is located in the posterior part of the cingulate gyrus (retrosplenial cortex), communicates with the temporal lobe (medial), is implicated in memory processing (Hirono et al., 1998) and is associated with the execution of novel tasks (Andreasen et al., 1995). Another region which was significantly activated during word learning was the medial frontal cortex (bilateral). Frontal cortex activations during long-term memory tasks possibly reflect control processes that support and optimize memory encoding and retrieval. The mid-dorsal region (Brodmann areas 9 and 46) is important for monitoring and maintaining pieces of information in working memory (Cabeza \& Nyberg, 2000). Mottaghy and colleagues (2002) showed that verbal working memory (2-back task) was impaired during rTMS stimulation of the medial frontal gyrus and the inferior parietal cortex (both bilaterally). It was suggested that the medial frontal gyrus functioned as a central executive system and that higher task complexity (2-back) was responsible for the right-sided involvement in the task (Mottaghy, Doring, Muller Gartner, Topper, \& Krause, 2002). Because frontal areas are a crucial link in the memory formation process, as they initiate the formation of the memory trace, it is to be expected that dysfunctions of these regions can result in defective memory formation (Kelley et al., 1998).

The present study also revealed a drug by task interaction. Subjects under influence of MDMA showed a similar increase in BOLD responses in the left medial frontal area, compared with placebo, in both the experimental and control condition. In the placebo condition, the fMRI response is increased in the experimental but not in the control condition. During placebo, it is to be expected that the fMRI response during the control list of pretreatment-memorized words be smaller compared with the fMRI response elicited by the experimental list, which contained new information that had to be memorized. The absence of a differential fMRI response to the experimental and the control list following MDMA is very noteworthy. It appeared that subjects under influence of MDMA invested an equal amount of effort in the experimental and the control list. This could be an indication for disturbed information transmission which could lead to impaired memory encoding.

The paradigm in the present study was previously used by other investigators, though in a slightly different form (Andreasen et al., 1995; Dupont et al., 2002). Dupont and colleagues (2002) investigated recall and encoding processes 
and found activation in 5 regions i.e. occipital cortex, left partietal cortex, left superior temporal cortex, right and left ventrolateral frontal cortex, and the fusiform gyrus. This activation was observed after subtraction of the experimental task-activation and the control task-activation. The present study mainly revealed bilateral activation in parietal and frontal regions, with significant differences between experimental task and control task in frontal regions (table 3). The contrast analysis of Dupont and colleagues (2002) and the analysis used in the present study disclose consistencies concerning the regions active during the encoding of words (frontal). The absence of significant differences in activation in the experimental list and control list in parietal or temporal regions during wordlearning in the present paradigm might be attributable due to the dissimilarity of both control tasks i.e. fixation of a single letter (Dupont et al., 2002) versus reading a list of 15 memorized words.

Quednow and colleagues (2006) have demonstrated defective memory in recently abstinent MDMA users with the same word-learning task as was used by Kuypers and Ramaekers (2005). They showed that although the learning curves had the same shape, MDMA users learnt less compared with two control groups i.e. a cannabis and a drug naïve group. Because they also found impairment of other memory related processes besides learning i.e. consolidation, recall, and recognition they concluded that it is not known where the impairment originates, although they suggest it is defective recall (Quednow et al., 2006). Ward and colleagues (2006) found that abstinent users were able to perform at the same level as a control group, and needed more learning to achieve comparable performance levels. Ward and colleagues ascribed memory problems of abstinent MDMA users to deficits in encoding (Ward, Hall, \& Haslam, 2006). In contrast to Quednow and colleagues who suggested that defective recall was the prime cause of impaired memory performance, this study shows that previously learnt words can perfectly be recalled under the influence of MDMA. This suggests that the inferior memory performance in MDMA users is rather due to defective or slower learning.

A general limitation of pharmaco-fMRI studies is that drugs may directly affect the BOLD response by vasodilatation or -constriction. Because MDMA acts generally on the serotonin (5-HT) system, and 5-HT has vasoconstrictor properties, it is possible that MDMA has a general effect on the cerebral blood flow (CBF) in the whole brain (Cohen, Bonvento, Lacombe, \& Hamel, 1996; Frackowiak, 2004; Meyer, Brevard, Piper, Ali, \& Ferris, 2006). The expected physiological effect of MDMA on CBF is a decrease in BOLD response (Brevard, Meyer, Harder, \& Ferris, 2006; Meyer et al., 2006). This would be general and 
independent of task-type. However, the fact that task effect, drug effect and drug by task effect in the brain activated selective areas in the brain counteracts the notion that brain activations in the present study were secondary to a general physiological effect of MDMA.

To conclude, a single dose of MDMA seemed to affect word-learning related structures as indicated by an increased BOLD response. This effect was reflected in the behavioral data showing subjects under the influence of MDMA to recall less compared with placebo, although with a sample size of 6 this was not found to be significant. Future analysis of the imaging data of the complete sample size will include random effects analysis (RFX) to examine the effects of drug and task on activity. 


\section{References}

Andreasen, N. C., O'Leary, D. S., Cizadlo, T., Arndt, S., Rezai, K., Watkins, G. L., et al. (1995). II. PET studies of memory: novel versus practiced free recall of word lists. NeuroImage, 2(4), 296-305.

Brevard, M. E., Meyer, J. S., Harder, J. A., \& Ferris, C. F. (2006). Imaging brain activity in conscious monkeys following oral MDMA ("ecstasy"). Magnetic resonance imaging, 24(6), 707-714.

Buckner, R. L., Kelley, W. M., \& Petersen, S. E. (1999). Frontal cortex contributes to human memory formation. Nature neuroscience, 2(4), 311-314.

Cabeza, R., \& Nyberg, L. (2000). Imaging cognition II: An empirical review of 275 PET and fMRI studies. Journal of cognitive neuroscience, 12(1), 1-47.

Cohen, Z., Bonvento, G., Lacombe, P., \& Hamel, E. (1996). Serotonin in the regulation of brain microcirculation. Progress in neurobiology, 50(4), 335-362.

Cusack, R., Cumming, N., Bor, D., Norris, D., \& Lyzenga, J. (2005). Automated post-hoc noise cancellation tool for audio recordings acquired in an MRI scanner. Human brain mapping, 24(4), 299-304.

de Wit, H., Enggasser, J. L., \& Richards, J. B. (2002). Acute administration of damphetamine decreases impulsivity in healthy volunteers. Neuropsychopharmacology official publication of the American College of Neuropsychopharmacology, 27(5), 813-825.

Dupont, S., Samson, Y., Le Bihan, D., \& Baulac, M. (2002). Anatomy of verbal memory: a functional MRI study. Surgical and radiologic anatomy SRA, 24(1), 57-63.

Frackowiak, R. S. (2004). Functional imaging of cognitive psychopharmacology. In R. S. Frackowiak (Ed.), Human brain function (second ed., pp. 303-327). Amsterdam: Elsevier Academic Press.

Gabrieli, J. D., Poldrack, R. A., \& Desmond, J. E. (1998). The role of left prefrontal cortex in language and memory. Proceedings of the National Academy of Sciences of the United States of America, 95(3), 906-913.

Hirono, N., Mori, E., Ishii, K., Ikejiri, Y., Imamura, T., Shimomura, T., et al. (1998). Hypofunction in the posterior cingulate gyrus correlates with disorientation for time and place in Alzheimer's disease. Journal of neurology, neurosurgery, and psychiatry, 64(4), 552-554.

Kelley, W. M., Miezin, F. M., McDermott, K. B., Buckner, R. L., Raichle, M. E., Cohen, N. J., et al. (1998). Hemispheric specialization in human dorsal frontal cortex and medial temporal lobe for verbal and nonverbal memory encoding. Neuron, 20(5), 927-936. 
Kuypers, K. P. C., \& Ramaekers, J. G. (2005). Transient memory impairment after acute dose of 75mg 3.4-Methylene-dioxymethamphetamine. Journal of psychopharmacology Oxford, England, 19(6), 633-639.

Meyer, J. S., Brevard, M. E., Piper, B. J., Ali, S. F., \& Ferris, C. F. (2006). Neural effects of MDMA as determined by functional magnetic resonance and magnetic resonance spectroscopy in awake marmoset monkeys. Annals New York academy of sciences, 1074, 365-376.

Mottaghy, F. M., Doring, T., Muller Gartner, H. W., Topper, R., \& Krause, B. J. (2002). Bilateral parieto-frontal network for verbal working memory: an interference approach using repetitive transcranial magnetic stimulation (rTMS). European journal of neuroscience, 16(8), 1627-1632.

Mulder-Hajonides van der Meulen, W. R. E. H., Wijnberg, J. R., Hollanders, J. J., DeDiana, I., \& Hoofdakker, R. (1980). Measurement of subjective sleep quality. Paper presented at the Fifth European Congress on Sleep Research, Amsterdam.

Ojemann, J. G., Buckner, R. L., Corbetta, M., \& Raichle, M. E. (1997). Imaging studies of memory and attention. Neurosurgery clinics of North America, 8(3), 307-319.

Quednow, B. B., Jessen, F., Kuhn, K. U., Maier, W., Daum, I., \& Wagner, M. (2006). Memory deficits in abstinent MDMA (ecstasy) users: neuropsychological evidence of frontal dysfunction. Journal of psychopharmacology Oxford, England, 20(3), 373-384.

Rey, A. (1958). The clinical examination in psychology / L'examen clinique en psychologie: Oxford, England: Presses Universitaries De France.

Schacter, D. L., \& Wagner, A. D. (1999). Medial temporal lobe activations in fMRI and PET studies of episodic encoding and retrieval. Hippocampus, 9(1), 7-24.

Smith, E. E., Jonides, J., Marshuetz, C., \& Koeppe, R. A. (1998). Components of verbal working memory: evidence from neuroimaging. Proceedings of the National Academy of Sciences of the United States of America, 95(3), 876-882.

Squire, L. R., \& Zola Morgan, S. (1991). The medial temporal lobe memory system. Science, 253(5026), 1380-1386.

Ward, J., Hall, K., \& Haslam, C. (2006). Patterns of memory dysfunction in current and 2year abstinent MDMA users. Journal of clinical and experimental neuropsychology official journal of the International Neuropsychological Society, 28(3), 306-324. 


\title{
CHAPTER 6
}

\section{ACUTE EFFECTS OF 3,4- METHYLENEDIOXYMETHAMPHETAMINE (MDMA) ON BEHAVIORAL MEASURES OF IMPULSIVITY, ALONE AND IN COMBINATION WITH ALCOHOL}

\author{
Ramaekers, J.G., and Kuypers, K.P.C.
}

Background. The use of 3,4-methylenedioxymethamphetamine (MDMA) has frequently been associated with increased levels of impulsivity during abstinence. The effects of MDMA on measures of impulsivity, however, have not yet been studied during intoxication. Objective. The present study was designed to assess the acute effects of MDMA and alcohol, alone and in combination, on behavioural measures of impulsivity and risk taking behaviour. Methods. A total of 18 recreational users of MDMA entered a double-blind placebo-controlled six-way crossover study. The treatments consisted of MDMA 0, 75, and $100 \mathrm{mg}$ with and without alcohol. Alcohol dosing was designed to achieve a peak blood alcohol concentration (BAC) of about $0.06 \mathrm{~g} / \mathrm{dl}$ during laboratory testing. Laboratory tests of impulsivity were conducted between 1.5 and $2 \mathrm{~h}$ post-MDMA and included a stop-signal task, a go/no-go task and the Iowa gambling task. Results. MDMA decreased stop reaction time in the stop-signal task indicating increased impulse control. Alcohol increased the proportion of commission errors in the stop-signal task and the go/no-go task. Signal detection analyses of alcohol-induced commission errors indicated that this effect may reflect impairment of perceptual or attentive processing rather than an increase of motor impulsivity per se. Performance in the Iowa gambling task was not affected by MDMA and alcohol, but there was a non-significant tendency towards improvement following alcohol intake. Conclusion. None of the behavioral measures of impulsivity showed a MDMA by alcohol interaction effect. The lack of interaction indicated that the CNS stimulant effects of MDMA where never sufficient to overcome alcoholinduced impairment of impulse control or risk-taking behavior.

Neuropsychopharmacology official publication of the American College of Neuropsychopharmacology, 2006; 31(5), 1048-1055 
92 | CHAPTER 6 


\section{Introduction}

3,4-Methylenedioxymethamphetamine (MDMA) is frequently used in combination with psychoactive drugs. Surveys in Australia (Topp, Hando, Dillon, Roche, \& Solowij, 1999) and Spain (Gamella, Alvarez-Roldan, \& Romo, 1997) have indicated that $40-60 \%$ of the MDMA users consumed alcohol concomitantly. Theories of drug use have proposed links with risk-taking behavior and impulsivity particularly among polydrug users (de Wit, Enggasser, \& Richards, 2002). Studies that have examined the effect of MDMA on impulsivity in abstinent MDMA users have provided conflicting results. McCann and colleagues (1994) reported a decrement in impulsivity ratings of MDMA users as measured by the Multidimensional Personality Questionnaire, whereas others reported elevated scores of impulsivity in heavy MDMA users, using different subjective measures of impulsivity (Butler \& Montgomery, 2004; Morgan, 1998, 2000; Parrott, 2000). Elevated levels of impulsiveness have been associated with lower levels of serotonin (5-HT) and CSF-5HIAA (Linnoila, Virkkunen, George, \& Higley, 1993; Linnoila et al., 1983) and with dopaminergic activation in the prefrontal cortex leading to a reduction in 'inhibitory control' over behavioral functions (Jentsch \& Taylor, 1999). MDMAinduced impulsivity could be accounted for by both mechanisms. Low CSF5HIAA levels have been observed in abstinent and recent MDMA users, whereas dopaminergic turnover is temporarily elevated during MDMA intoxication (Green, O'Shea, \& Colado, 2004; Nash, 1990).

Research on MDMA-related impulsivity has generally relied on subjective questionnaires and self report, which have provided valuable information about impulsivity as a trait in abstinent MDMA users. It has been argued, however, that these measures may not be very well suited for pharmacological studies of impulsivity because they are subjective, measure a relatively stable characteristic, and cannot be directly related to biological models of impulsivity (Swann, Bjork, Moeller, \& Dougherty, 2002). These problems have been overcome by using behavioral measures of impulsivity as defined by the inability to anticipate and reflect on the consequences of decision making or by the failure to inhibit a response in a rapid response task (Bechara, Damasio, \& Damasio, 2000; Logan, Cowan, \& Davis, 1984). Studies in MDMA research employing behavioral tests have been relatively sparse but those that were conducted seem to support subjective reports of enhanced impulsivity. Heavy MDMA users produced more commission errors in a go/no-go task (Moeller et al. 2002) and made more risky choices in a gambling task (Butler \& Montgomery, 2004) when compared to nondrug users. In addition, current and former MDMA users produced faster 
responses and more incorrect choices in a matching familiar figures test designed to measure reflection-impulsiveness (Morgan, 1998).

Yet, despite mounting evidence that inhibitory control may be impaired in abstinent MDMA users, the discussion as to its underlying cause remains controversial. A number of confounding factors have been suggested that may account for increments in risk-taking or impulsivity observed in MDMA users, besides or instead of MDMA use per se. For example, MDMA users are generally polydrug users of a range of recreational drugs, which makes attribution of impulsivity to either one drug virtually impossible. In addition, it cannot be excluded that impulse and risk-taking behaviors are related to pre-existing low levels of serotonin in MDMA users or that a predisposition to elevated impulsivity underlies the use of MDMA or self-reports and laboratory results from studies in abstinent MDMA users (Butler \& Montgomery, 2004; Cole \& Sumnall, 2003). Consequently, a better approach for determining a causal relation between MDMA use and change in impulse control may be to conduct placebo-controlled studies on the acute effects of MDMA on laboratory measures of impulse control. Acute studies will be crucial for showing a direct pharmacological link between MDMA use and impulse control during intoxication and may even provide a mechanistic blueprint for MDMA-induced long-term changes in impulse control.

The aim of the present study is to assess the acute effect of MDMA alone and in combination with alcohol on impulsivity in a double-blind, placebocontrolled, within-subject design. Impulsivity was either defined as the failure to inhibit a response in rapid-response models (go/no-go task and stop-signal task) or as the inability to anticipate and reflect on the consequences of a decision (Iowa gambling task). It was predicted that a general increase in brain 5-HT following a single dose of MDMA would improve impulse control. Alcohol has been previously shown to decrease impulse control in rapid-response tasks (de Wit, Crean, \& Richards, 2000; Fillmore \& Vogel Sprott, 1997, 1999) and was included in the present study design as an active control and to assess potential MDMA by alcohol interaction effects.

\section{Methods}

\section{Subjects}

A total of 18 recreational MDMA users (nine males, nine females) aged 20-37 years were recruited through advertisements in local newspapers. All subjects were lightto-moderate users of MDMA who reported to have taken the drug on 2-25 
occasions (mean: 9 occasions) in the previous year. Overall, subjects reported to have taken between 2 and 120 MDMA tablets (mean: 18 tablets) in the previous year. Initial screening was accomplished on the basis of a questionnaire on medical history. Subjects who were accepted were examined by the medical supervisor, who also checked vital signs and took blood and urine samples. Standard blood chemistry, haematological and drug screen tests were conducted on these samples. Inclusion criteria were: experience with the use of MDMA; free from psychotropic medication; good physical health as determined by examination and laboratory analysis; absence of any major medical, endocrine, and neurological condition; normal weight, body mass index (weight/height ${ }^{2}$ ) between 18 and $28 \mathrm{~kg} / \mathrm{m}^{2}$; and written informed consent. Exclusion criteria were: history of drug abuse (other than the use of MDMA) or addiction; pregnancy or lactation; cardiovascular abnormalities as assessed by standard 12-lead ECG; excessive drinking ( $>20$ standard alcoholic consumptions a week); hypertension (diastolic $>100 \mathrm{mmHg}$; systolic $>170 \mathrm{mmHg}$ ); and history of psychiatric or neurological disorder.

This study was conducted according to the code of ethics on human experimentation established by the declaration of Helsinki (1964) and amended in Edinburgh (2000). All subjects gave their informed consent, in writing. Approval for the study was obtained from the University's Medical Ethics committee. A permit for obtaining, storing and administering MDMA was obtained from the Dutch drug enforcement administration.

\section{Design, doses and administration}

The study followed a double-blind, placebo-controlled, six-way crossover design. Complete balancing of the treatment orders yielded 6 treatment orders randomly assigned to 18 subjects. The treatments consisted of MDMA 0, 75, and $100 \mathrm{mg}$ with and without alcohol. MDMA and MDMA-placebo were administered orally in identically appearing formulations. MDMA was administered as a $25 \mathrm{ml}$ solution in bitter orange peel syrup, which was ingested at once. Alcohol dosing was designed to achieve a peak BAC of about $0.06 \mathrm{~g} / \mathrm{dl}$ during laboratory testing. Alcohol doses were administered at 45 minutes post-drug, and consumed within a period of 15 minutes. Subjects' BAC was monitored regularly at 15-minute intervals for 30-90 minutes after cessation of drinking using a Lion SD-4 Breath Alcohol Analyzer. The minimum wash-out period between successive treatments was 1 week. Laboratory tests were conducted between 1.5 and $2 \mathrm{~h}$ post-drug (i.e. 0.5 and $1 \mathrm{~h}$ post alcohol). 


\section{Procedures}

Subjects were asked to refrain from any drugs starting 1 week before the medical screening and physical examination until 2 weeks after the last experimental session. The subjects were not allowed to use alcohol on the day prior to an experimental session and were requested to arrive well rested at the experimental sessions. Drug and alcohol screens in breath and urine were performed prior to experimental sessions upon arrival of the subject. Drugs and placebo were only administered in case a subject had passed the urine drug screen on a given test day. Three subjects tested positive for cannabis in urine prior to drug of placebo administration. These subjects were all sent home to return to our laboratory at a later time. Subjects were transported from their homes to the laboratory or vice versa by one of the experimenters in order to prevent subjects from driving under the influence on the day of testing. Additional clinical blood chemistry, with particular reference to liver and renal function was conducted at day 7 after each treatment. All subjects received a training session prior to onset of the experimental sessions in order to familiarize them with the tests and procedures.

\section{Behavioural tests of impulsivity}

Stop-signal task. This task requires subjects to make quick key responses to visually presented go-signals and to inhibit any response when a visual stop-signal is suddenly presented. The current test is adapted from an earlier version of Fillmore et al (Fillmore, Rush, \& Hays, 2002). The go-signals are four $1.5 \mathrm{~cm}$ letters (A, B, $\mathrm{C}$, and D) presented one at a time in the center of a computer screen. Subjects are required to respond to each letter as quickly as possible by pressing one of two response buttons. One button is pressed to indicate that ' $\mathrm{A}$ ' or ' $\mathrm{C}$ ' appeared and the other to indicate 'B' or 'D'. Letters are displayed for 500 milliseconds and the computer screen is blank for 1.5 seconds (inter-stimulus-interval) before the next letter is displayed. This provides a period of 2 seconds in which the subject can respond to a letter.

A single test consists of 176 trials in which each of the four-letter stimuli is presented equally often. A stop signal occurs in 48 trials during the test. The stopsignal consists of visual cue, i.e. "*”, that appears in one of the four corners of the screen. Subjects are required to withhold any response in case a stop-signal is presented. Stop-signals are presented 12 times at each of the four delays i.e. 50, 150, 250 and 350 milliseconds after the onset of a letter. Trials always begin with a 500-milliseconds preparation interval in which a fixation point appears in the center of the screen. The task lasts for about 10 minutes. Dependent variables are the proportion of commission errors on stop-trials and the reaction times (RT) on 
go and stop-signal trials (i.e. stop reaction time). Stop reaction time to stop-signal trials represents the estimated mean time required to inhibit a response.

The method for calculating stop reaction time was taken for the race model of inhibitory control (Logan, 1994). This model proposes that the response to stop-signal trials is defined by two parallel processes: execution of a motor action in response to a signal and inhibition of a motor action in response to stopsignal. Crucial to the outcome of the race is the speed of both processes. Response inhibition will fail if the time required to inhibit exceeds the time to complete a motor response at the time of the stop-signal. The speed of the inhibition response cannot be observed directly but can be derived mathematically on the basis of three factors: stop-signal delay, reaction time distribution on go-trials and the probability of successful response inhibitions in stop-signal trials. First, reaction times to 128 go-trials were rank-ordered from shortest to longest. The finishing time of the inhibition response was then determined from the probability of successful response inhibition and the distribution of RT. If $n \%$ of the responses on stop-signal trials would be unsuccessfully inhibited (i.e. commission error), than the finishing time would be associated with the $n$th percentile of the RT distribution. Stop reaction time was then determined by subtracting the appropriate stop-signal delay from reaction time at the $n$th percentile of the RT distribution. The resulting values for each stop-signal delay were then averaged to yield a single measure of stop reaction time for the test.

Go/no-go task. The Go/no-go task provides a measure of the ability to inhibit or suppress a habit response. During the test, a series of letters (A, E, H, L, K and X) appear one at a time in the center of the computer screen, and participants are instructed to press the spacebar at the appearance of each letter (go condition). However, if the letter ' $\mathrm{X}$ ' is preceded by 'A', subjects have to inhibit their prepared responses (no-go condition). Dependent variables include average RT on go trials and errors of commission; that is, pushing the spacebar during no-go trials. In addition, the signal detection theory model (Green \& Swets, 1966) was applied to assess the subjects' operation characteristics during task performance. Hit rate $(H$, probability of response inhibition on no-go trials) and false alarm rate ( $F A$, probability of response inhibition on go trials) were established to determine the main parameters of the signal detection model; that is sensitivity (d') and response criterion $(\beta)$. Hit and false alarm rates were referred to standardized normal distributions in order to yield $z(H)$ and $z(F A)$. The sensitivity index $d$ ' is given by $z(H)-z(F A)$ expressed in normal deviates. The response criterion $\beta$ is conceptualized as $f(H) / f(\mathrm{~F} A)$, where $f(H)$ and $f(\mathrm{~F} A)$ are the ordinates of the normal 
curve corresponding to $H$ and $F A$. Sensitivity reflects the discriminability of go trials from no-go trials, whereas the response criterion indicates the minimum level of certainty that is necessary for the subject to make a 'no-go' decision. Two respondents may differ considerably in their criterion, even when they are equally sensitive. A subject's response criterion may be liberal or risky, thereby detecting most of the no-go signals at the cost of many false alarms, or it may be conservative, leading to few false alarms while reducing the probability of detecting no-go signals.

The Iowa gambling task. The subject sees four decks of cards on a computer screen labeled A, B, C, and D at the top end of each deck. With a mouse, the subject can click on a card on any of the four decks. Each deck of cards is programmed to have 40 cards. The gains and losses for each card selection are set so that in each block of 10 cards from deck A or deck B over the course of trials there is a total gain of $\$ 1000$ (interspersed with unpredictable losses totalling $\$ 1250$ ). For decks $C$ and $\mathrm{D}$, the gains and losses for each card selection are set so that in each block of 10 cards there is a total gain of $\$ 500$, interrupted by losses totaling $\$ 250$ (gains and losses all refer to virtual money). Thus decks $\mathrm{A}$ and $\mathrm{B}$ are 'disadvantageous' in the long-term, while decks $\mathrm{C}$ and $\mathrm{D}$ are 'advantageous' in the long-term. The majority of normal people choose advantageously on this task i.e. they select more cards from the advantageous relative to the disadvantageous decks. Patients with frontal lobe lesions do the opposite, that is, select more disadvantageous cards. The sensitivity, reliability, and validity of this task in detecting decision-making impairments has been tested in neurological as well as psychiatric populations. Thus there is one dependent measure that we collect from this task: net score (total number of cards picked from $\mathrm{C}$ and $\mathrm{D}$ minus total number of cards picked from $\mathrm{A}$ and $\mathrm{B})$. Parallel versions of the gambling task are used over six treatment sessions (Bechara et al., 2000; Bechara et al., 2001).

\section{Pharmacokinetic assessments}

MDMA was determined in plasma $(15 \mathrm{ml})$ at $1.5 \mathrm{~h}$ post-drug. Blood samples were placed on ice immediately, centrifuged later, and frozen at minus $20^{\circ} \mathrm{C}$ until analyses for pharmacokinetic assessments. MDMA and MDA concentrations were determined using solid-phase extraction and gas chromatography with mass spectrometric detection with quantification limits of $1 \mathrm{ng} / \mathrm{ml}$. BAC concentrations were assessed every 15 minutes during the first 2 hours after drinking with a Lion SD-4 Breath Alcohol Analyzer. 


\section{Statistics}

Data were analyzed by means of repeated measures MANOVA with Alcohol (two levels) and MDMA (three levels) and their interaction as main factors. Separate drug-placebo contrasts were conducted following an overall effect of MDMA or the interaction between MDMA and Alcohol. The alpha criterion level of significance was set at $p=0.05$.

\section{Results}

Complete data sets $(N=18)$ were collected for the Iowa gabling task and the go/no-go task. In case of the stop-signal task, incomplete data sets were recorded from one subject due to technical problems. Only complete data sets entered the statistical analyses.

On the stop-signal task, MANOVA indicated a significant overall effect of MDMA on stop reaction time $\left(\mathrm{F}_{2,15}=4.62 ; p=0.027\right)$. In general, MDMA decreased stop reaction time as compared to placebo treatments. Separate drug-placebo contrasts indicated that this effect was particularly noteworthy for MDMA $75 \mathrm{mg}$ $(p=0.008)$. Mean (SE) stop reaction times in every treatment condition are shown in Figure 1 (See also Table 1).

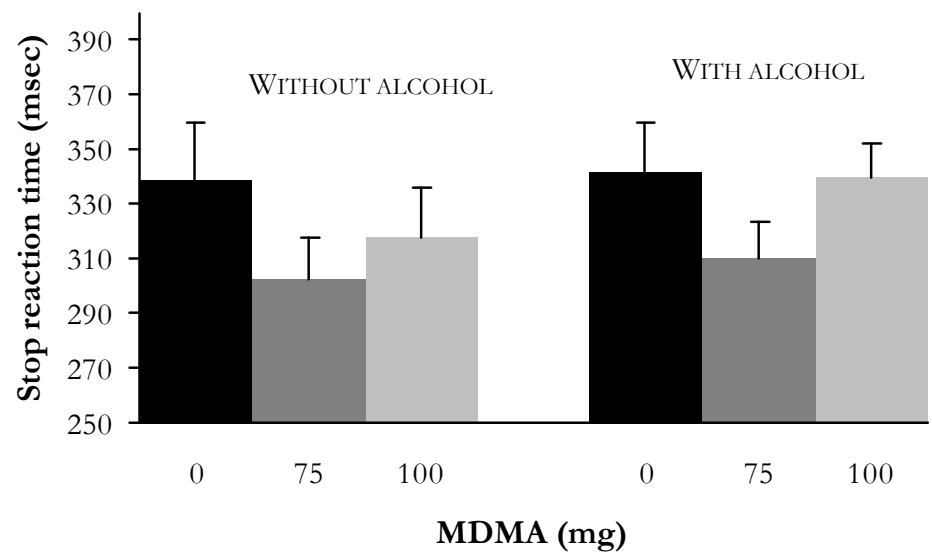

Figure 1. Mean (SE) Stop Reaction Times in each treatment condition in the Stop-signal task

MANOVA also revealed significant main effects of alcohol on the percentage of commission errors in the stop-signal task $\left(\mathrm{F}_{1,16}=7.06 ; p=0.017\right)$ and the go/no-go task $\left(F_{1,17}=7.84 ; p=0.012\right)$. During alcohol treatments the proportion of commission errors was significantly higher as compared to 
treatments without alcohol in both tests. Mean proportions of commission errors in all treatment conditions in the stop signal task and the go/no-go task are shown in Figure 2 and 3. Signal detection parameters related to performance at the go/no-go task indicated that the increment in commission errors during alcohol was related to a reduction in the subjects' sensitivity $(d)$ to distinguish go from nogo signals $\left(F_{1,17}=18.25 ; p=0.001\right)$, and not to a change in the subjects' response criterion $(\beta)$. Mean (SE) sensitivity in each treatment condition is shown in Figure 4 (See also Table 1).

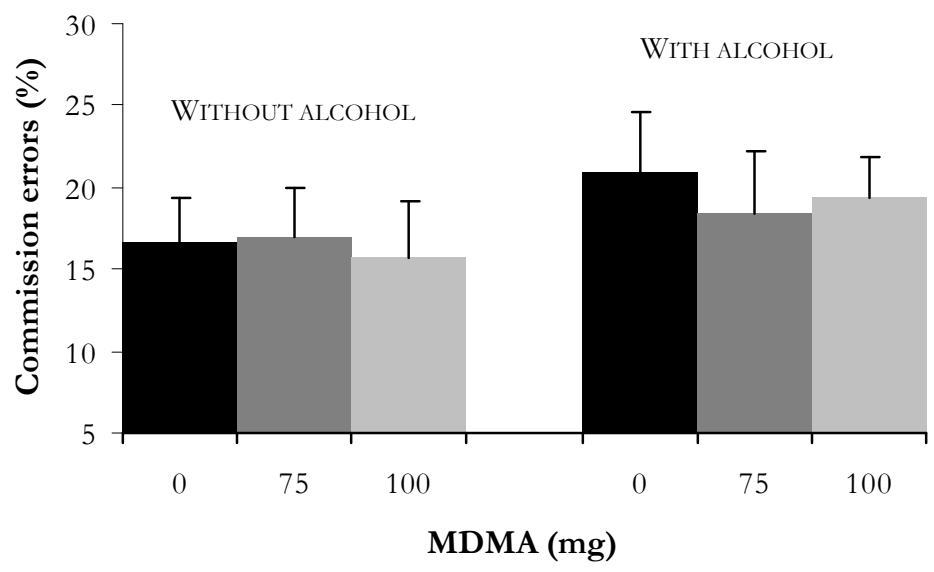

Figure 2. Mean (se) proportions of commission errors during each treatment condition in the Go/no go Task

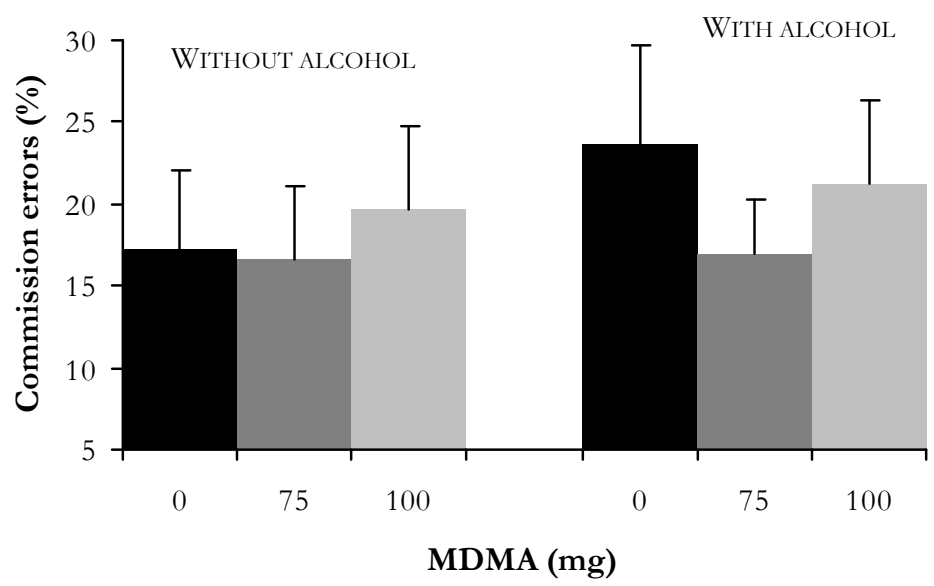

Figure3. Mean (se) proportions of commission errors during each treatment condition in the Stop Signal Task 


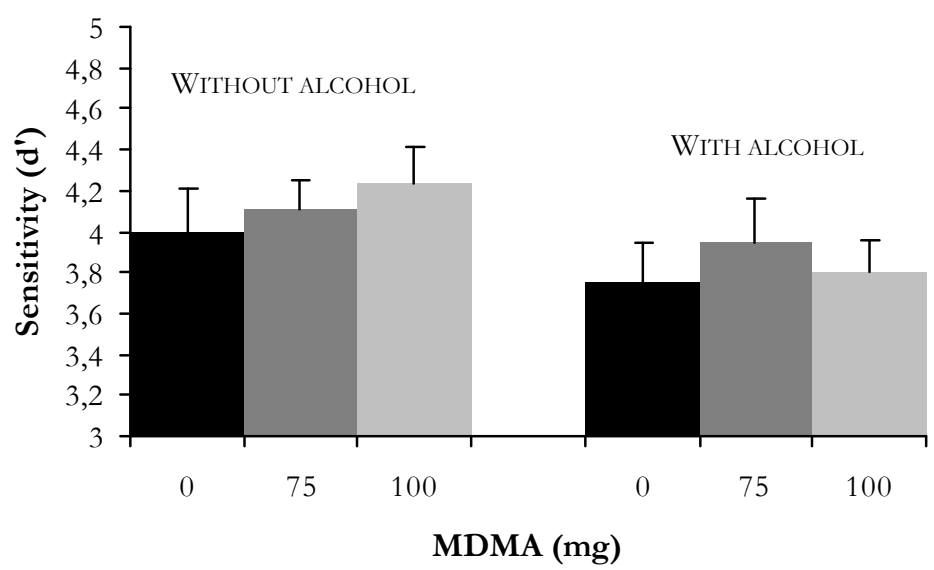

Figure 4. Mean (SE) sensitivity (d') in each treatment condition in the Go/No-Go task.

In the gambling task, alcohol tended to improve performance as indicated by an almost significant main effect of alcohol $\left(\mathrm{F}_{1,17}=3,14 ; p=0.09\right)$. On average, the number of advantageous cards selected during alcohol treatments, were higher as compared to treatments without alcohol. MDMA did not affect performance in the gambling task. There were no alcohol-by-MDMA interactions on any behavioral measures of impulsivity (Table 1).

\section{Pharmacokinetics}

Mean BAC did not significantly differ between treatments. Mean (SE) BACs at the onset of laboratory testing, that is, 30 minutes post drinking were, respectively, $0.65(0.19), 0.56(0.12)$ and $0.57(0.16) \mathrm{mg} / \mathrm{ml}$, following placebo, MDMA 75 and $100 \mathrm{mg}$. Mean (SE) BACs at the end of laboratory testing, that is, 60 minutes post drinking were, respectively, 0.64 (0.16), 0.59 (0.11) and 0.59 (0.12) $\mathrm{mg} / \mathrm{ml}$ following placebo, MDMA $75 \mathrm{mg}$ and MDMA $100 \mathrm{mg}$.

MDMA and MDA concentrations were also comparable between treatments. At onset of the laboratory test, that is, $1.5 \mathrm{~h}$ post-drug, mean (SE) MDMA concentrations were 137.4 (31.9), 191.8 (49.1) ng/ml following MDMA 75 $\mathrm{mg}$ and $100 \mathrm{mg}$, respectively. When combined with alcohol, MDMA concentrations following the $75 \mathrm{mg}$ and $100 \mathrm{mg}$ dose were 147 (36.3) and 208.5 (45.7), respectively. MDA concentrations following single doses of MDMA 75, MDMA 100 alone and combined with alcohol were, respectively, 3.5 (1.2), 4.5 (1.5), 3.4 (1.0), and 4.7 (1.4) ng/ml. 
Table 1. Mean (se) values of all laboratory measures of impulsivity in every treatment condition and a summary of significant treatment effects of $M D M A$, alcohol and their interaction as indicated by $M A N O V A$

\begin{tabular}{|c|c|c|c|}
\hline Test & Placebo & $\begin{array}{c}\text { TREATMENT } \\
\text { Without Alcohol } \\
\text { MDMA } 75\end{array}$ & MDMA 100 \\
\hline \multicolumn{4}{|l|}{ STOP SignAL TASK } \\
\hline Stop RT (msec) & $339(21)$ & $303(15)$ & $318(18)$ \\
\hline Go RT (msec) & $723(60)$ & $648(36)$ & $651(39)$ \\
\hline Commission errors $(\%)$ & $17,2(4.6)$ & $16,7(4,4)$ & $19,6(5,1)$ \\
\hline \multicolumn{4}{|l|}{ Go/NO GO TASK } \\
\hline Go RT (msec) & $282(15)$ & $287(16)$ & $294(20)$ \\
\hline Commission errors $(\%)$ & $16,6(2,7)$ & $17,0(2,9)$ & $15,7(3,4)$ \\
\hline Sensitivity (d') & $4.0(0.2)$ & $4.1(0.1)$ & $4.2(0.2)$ \\
\hline Response criterion ( $(\beta)$ & $3.4(0.2)$ & $3.9(0.2)$ & $3.7(0.2)$ \\
\hline \multicolumn{4}{|l|}{ IOWA GAMBLING TASK } \\
\hline \multirow[t]{2}{*}{ Ratio good/bad } & $-10,7(2,8)$ & $-4,2(3,0)$ & $-3,3(4,9)$ \\
\hline & Placebo & $\begin{array}{l}\text { With Alcohol } \\
\text { MDMA } 75\end{array}$ & MDMA 100 \\
\hline \multicolumn{4}{|l|}{ STOP SigNAL TASK } \\
\hline Stop RT (msec) & $342(18)$ & $310(14)$ & $340(12)$ \\
\hline Go RT (msec) & $658(42)$ & $655(29)$ & $663(37)$ \\
\hline Commission errors $(\%)$ & $23,7(6,1)$ & $16,9(3,4)$ & $21,3(5,1)$ \\
\hline \multicolumn{4}{|l|}{ GO/NO GO TASK } \\
\hline Go RT (msec) & $287(15)$ & $278(14)$ & $288(16)$ \\
\hline Commission errors $(\%)$ & $21.0(3,6)$ & $18,4(3,7)$ & $19,8(2,4)$ \\
\hline Sensitivity (d') & $3.7(0.2)$ & $3.9(0.2)$ & $3.8(0.2)$ \\
\hline Response criterion ( $($ ) & $3.5(0.3)$ & $3.3(0.3)$ & $3.6(0.3)$ \\
\hline \multicolumn{4}{|l|}{ IOWA GAMBLING TASK } \\
\hline \multirow[t]{2}{*}{ Ratio good/bad } & $1,6(4,9)$ & $-3,9(3,1)$ & $1,8(5,0)$ \\
\hline & MDMA & $\begin{array}{c}\text { MANOVA } \\
\text { A }\end{array}$ & MDMA*A \\
\hline \multicolumn{4}{|l|}{ STOP SignAL TASK } \\
\hline Stop RT (msec) & 0.027 & - & - \\
\hline Go RT (msec) & - & - & - \\
\hline Commission errors $(\%)$ & - & 0.012 & \\
\hline \multicolumn{4}{|l|}{ Go/NO GO TASK } \\
\hline Go RT (msec) & - & - & - \\
\hline Commission errors $(\%)$ & - & 0.013 & - \\
\hline Sensitivity (d') & - & 0.001 & - \\
\hline Response criterion (B) & - & - & - \\
\hline \multicolumn{4}{|l|}{ IOWA GAMBLING TASK } \\
\hline Ratio good/bad & - & - & - \\
\hline
\end{tabular}

\section{Discussion}

The main findings of this study on impulsivity were (1) that acute doses of MDMA improved impulse control in the stop-signal task; (2) that a moderate dose of alcohol impaired the subjects' ability to inhibit responses in the stop-signal and go/no-go paradigms; and (3) that MDMA did not affect alcohol-induced impairment in response inhibition tasks.

The present study has been the first to assess the acute effects of MDMA on behavioral measures of impulsivity. In two tasks, that is, the stop-signal task 
and the go/no-go task, impulsivity was defined as the inability to inhibit an activated or pre-cued response leading to errors of commission. In the third task, that is, the Iowa gambling task, impulsivity was defined as the inability to anticipate and reflect on the consequences of decision making. In both response inhibition tasks, MDMA did not affect the proportion of commission errors but significantly improved stop reaction time in the stop-signal task. These data indicate that a single dose of MDMA has the potential to stimulate inhibitory motor control in a stop-signal task during intoxication. The pharmacological mechanism underlying improvement in impulse control may be related to acute serotonergic suppletion following a single dose of MDMA. MDMA is believed to increase 5-HT levels during intoxication by stimulating the acute release of 5-HT and by blocking the pre-synaptic reuptake of 5-HT (Steele, McCann, \& Ricaurte, 1994; Steele, Nichols, \& Yim, 1987). Improved impulse control during serotonergic suppletion seems in line with reports on impaired impulse control in heavy users of MDMA during periods of abstinence. Studies on serotonergic function in recreational MDMA users have repeatedly shown low levels of 5HIAA in CSF (Bolla, McCann, \& Ricaurte, 1998; McCann et al., 1994; Peroutka, Pascoe, \& Faull, 1987; Ricaurte, Finnegan, Irwin, \& Langston, 1990), reduced density of 5-HT transporters (Reneman et al., 2002) and blunted neuro-endocrine responses in serotonergic challenge tests (Gerra et al., 1998; McCann, Eligulashvili, Mertl, Murphy, \& Ricaurte, 1999; Verkes et al., 2001) when compared to nondrug using controls. In addition, serotonergic depletion has been linked to behaviors characterized by impaired impulse control (Linnoila et al., 1993; Linnoila et al., 1983). Thus, acute improvement of inhibitory control in the stop-signal task following MDMA administration can be interpreted as supportive evidence for current notions on the link between serotonin and impulsivity. Impulsivity levels may drop following serotonin suppletion during acute MDMA intoxication but rise during periods of abstinence when serotonin levels are low.

Alternatively, the reduction in stop reaction time during MDMA intoxication may have also resulted from increased dopaminergic neurotransmission. MDMA is known to also stimulate the release of dopamine and to bind to the presynaptic dopamine reuptake transporters, albeit with less affinity then for the 5-HT transporter (Green et al., 2004). Studies on the effects of dopamine agonists on behavioral measures of impulsivity have shown patterns of improvement in impulse control that are similar to the one presented here, following MDMA. D-amphetamine for example, was shown to decrease stop reaction time of healthy volunteers in a stop-signal paradigm without affecting 
reaction time to go trials (de Wit et al., 2000; de Wit et al., 2002). However, opposite effects of amphetamine on behavioral tests of impulsivity have also been found when tested in long-term stimulant abusers. Fillmore and colleagues (2003) reported that an acute dose of d-amphetamine produced a dose-dependent increase in inhibition failures in long-term stimulant abusers performing a cued go/no-go task. Similarly, single doses of cocaine reduced the ability to inhibit responses of cocaine abusers in a stop-signal task (Fillmore et al., 2002). It has been suggested that repeated dopaminergic stimulation of prefrontal pathways leads to impairment of inhibitory functions (Volkow, Wang, \& Fowler, 1997; Volkow, Wang, Fowler et al., 1997) and that neural changes following chronic stimulant use may alter the behavioral response to acute dopaminergic stimulation (Fillmore, Kelly, \& Martin, 2005). In the present study, the subjects can be best qualified as light-to-moderate MDMA users. Therefore, subject characteristics in the present study would seem more comparable to those in healthy volunteer studies than to those in studies employing long-term drug abusers.

MDMA did not affect measures of impulsivity in every behavioral task employed in the present study. Decision making processes in the Iowa gambling task were not affected by MDMA which seems to indicate that its effect on stop reaction time in the stop-signal task is rather selective. It has been argued before that impulsivity is a broad conceptual construct that encompasses multiple mechanisms of behavioral control that may be linked to different regions of the brain (Bechara \& Martin, 2004). Two types of impulsivity that can be distinguished are cognitive impulsivity and motor impulsivity. Cognitive impulsivity as measured by the Iowa gambling task is believed to reflect complex processes involved in the control of several cognitive, behavioral and affective processes. More in particular, it assesses the ability to think and reflect on the consequences of a choice prior to making a decision. The ventromedial section in the prefrontal cortex has been indicated as the most critical neural structure underlying these processes (Bechara, 2004; Ridderinkhof, van den Wildenberg, Segalowitz, \& Carter, 2004). Motor impulsivity or response inhibition as measured in the stop-signal task or go/no-go task on the other hand, is believed to relate to the control of motor processes that are subserved by the anterior cingulate (Bechara, 2004). Thus, it is entirely possible that a pharmacological manipulation can affect these control systems independently and selectively. The present study thus suggests that MDMA selectively improves control over motor impulsivity, while leaving cognitive impulsivity intact. It is interesting to note that selectivity of MDMA effects have been reported in other psychological domains as well. Lamers and colleagues 
(2003) demonstrated that a single dose of MDMA produced dissociable effects on psychomotor skills and attention (Lamers et al., 2003).

The effects of alcohol on measures of behavioral impulsivity were intriguing. Alcohol reduced performance in the stop-signal and go/no-go tasks but almost significantly improved performance in the Iowa gambling task. Similar findings have been reported in the scientific literature. Previous studies employing stop-signal paradigms have reported that alcohol increased stop reaction time (de Wit et al., 2000) or increased the number of commission errors (Fillmore \& Vogel Sprott, 1997, 1999; Mulvihill, Skilling, \& Vogel Sprott, 1997), suggesting increased impulsivity. Application of signal detection theory to data from the go/no-go task in the present study indicated that the increase in commission errors during alcohol intoxication did not result from a change in response bias toward more liberal or risky decisions, but from a reduced ability to discriminate go from no-go signals. Or in terms of signal detection parameters: sensitivity $\left(d^{\prime}\right)$ significantly decreased following alcohol treatment, whereas the response criterion $(\beta)$ was unaffected. Sensitivity is a measure of sensory capabilities of the observer or of the effective signal strength, whereas response criterion is a measure of cautiousness and reflects such things as motives and attitudes. The present data thus suggest that the increase in commission errors may reflect impairment of perceptual or attentive processing rather than an increase of motor impulsivity. The absence of an alcohol effect on reaction time in go and no-go trials in the stop-signal task as well as the go/no-go task further supports this notion. It should be noted, however, that overall mean values for sensitivity and response criterion were rather high in the present experiment, which indicates that the go/no-go task was rather easy to perform by the subjects. It would be preferable to increase task difficulty in future studies to further assess the contribution of alcohol-induced changes in sensitivity and response criterion to performance under more compelling laboratory circumstances.

Performance on the Iowa gambling task tended to improve following alcohol treatment but this effect only approached statistical significance. It is still noteworthy, however, since similar effects have been reported in models, where cognitive impulsivity is defined as the inability to wait for a larger reward. Ortner and colleagues (2003) employed a delay-discounting task, where healthy volunteers made a series of hypothetical choices between a small, immediate reward and a large, delayed reward. In the alcohol condition, subjects tended to discount delayed reward at lower rates than during the sober condition. This difference was not statistical significant, but suggested that alcohol led to more cautious decision 
making. Performance stimulating effects were not found by Richards and colleagues (1999) but they did show that alcohol did not detrimentally affect discounting of delayed monetary reward. Although conceptual differences between tasks measuring cognitive impulsivity do exist, it has been shown that performance on delay-discounting tasks is significantly correlated to performance on the Iowa gambling task (Monterosso, Ehrman, Napier, O'Brien, \& Childress, 2001). Together these results indicate that alcohol does not increase cognitive impulsivity in this type of decision-making tasks. The absence of an alcohol effect on decision making tasks versus the presence of a detrimental effect of alcohol on motor inhibition tasks demonstrates once more that impulsivity is not a unitary concept or brain structure but is comprised of several independent psychological domains or brain regions that can respond very selectively to a pharmacological manipulation.

None of the behavioral parameters showed any significant MDMA by alcohol interaction effect. This suggests that MDMA does not alter the effects of alcohol on behavioral measures of impulsivity. This notion seems in line with conclusions from a previous study that assessed combined effects of alcohol and MDMA on psychomotor function during intoxication in humans (Hernandez Lopez et al., 2002). These investigators reported that MDMA reversed the subjective sedation induced by alcohol but did not change the impairing effect of alcohol on measures of psychomotor function such as simple reaction time and digit symbol substitution. The lack of mitigating effects of MDMA on alcoholinduced impairment on measures of impulsivity or risk-taking may be of particular importance in terms of road safety issues. Most of the MDMA-impaired driving cases that have been reported in the scientific literature consist of drivers who have taken multiple drugs and/or alcohol (Logan \& Couper, 2001). The present data indicates that the CNS-stimulating effects of MDMA do not suffice to overcome alcohol-induced impairment of motor control, which is one of the most common causal factors in vehicle crashes.

\section{Acknowledgments}

We would like to thank Gert De Boeck and Marleen Laloup from NICC, Brussels, for analyzing MDMA blood plasma samples. We also would like to thank Kirsten Schuer, Lisa Willems for their relative contribution to the study. This work was conducted as part of the IMMORTAL research consortium funded by the European Commission (Grant GMA12000-27043). 


\section{References}

Bechara, A. (2004). Separate neural substrates underlie different mechanisms of performance monitoring and behavioral control. In M. Ullsperger \& M. Falkenstein (Eds.), Errors, conflicts and the brain. Current opinions on performance monitoring (MPI special issue in buman cognitive and brain sciences) (pp. 55-62). Leipzig: Max Planck-Institute fur Kognitions und Neurowissenschaften.

Bechara, A., Damasio, H., \& Damasio, A. R. (2000). Emotion, decision making and the orbitofrontal cortex. Cerebral cortex New York, N.Y. 1991, 10(3), 295-307.

Bechara, A., Dolan, S., Denburg, N., Hindes, A., Anderson, S. W., \& Nathan, P. E. (2001). Decision-making deficits, linked to a dysfunctional ventromedial prefrontal cortex, revealed in alcohol and stimulant abusers. Neuropsychologia, 39(4), 376-389.

Bechara, A., \& Martin, E. M. (2004). Impaired Decision Making Related to Working Memory Deficits in Individuals With Substance Addictions. Neuropsychology, 18(1), 152-162.

Bolla, K. I., McCann, U. D., \& Ricaurte, G. A. (1998). Memory impairment in abstinent MDMA ("Ecstasy") users. Neurology, 51(6), 1532-1537.

Butler, G. K., \& Montgomery, A. M. (2004). Impulsivity, risk taking and recreational 'ecstasy' (MDMA) use. Drug and alcohol dependence, 76(1), 55-62.

Cole, J. C., \& Sumnall, H. R. (2003). Altered states: the clinical effects of Ecstasy. Pharmacology and Therapeutics, 98, 35-58.

de Wit, H., Crean, J., \& Richards, J. B. (2000). Effects of d-amphetamine and ethanol on a measure of behavioral inhibition in humans. Behavioral neuroscience, 114(4), 830-837.

de Wit, H., Enggasser, J. L., \& Richards, J. B. (2002). Acute administration of damphetamine decreases impulsivity in healthy volunteers. Neuropsychopharmacology official publication of the American College of Neuropsychopharmacology, 27(5), 813-825.

Fillmore, M. T., Kelly, T. H., \& Martin, C. A. (2005). Effects of d-amphetamine in human models of information processing and inhibitory control. Drug and alcohol dependence, 77(2), 151-159.

Fillmore, M. T., Rush, C. R., \& Hays, L. (2002). Acute effects of oral cocaine on inhibitory control of behavior in humans. Drug and alcohol dependence, 67(2), 157-167.

Fillmore, M. T., Rush, C. R., \& Marczinski, C. A. (2003). Effects of d-amphetamine on behavioral control in stimulant abusers: the role of prepotent response tendencies. Drug and alcohol dependence, 71(2), 143-152.

Fillmore, M. T., \& Vogel Sprott, M. (1997). Resistance to cognitive impairment under alcohol: the role of environmental consequences. Experimental and clinical psychopharmacology, 5(3), 251-255. 
Fillmore, M. T., \& Vogel Sprott, M. (1999). An alcohol model of impaired inhibitory control and its treatment in humans. Experimental and clinical psychopharmacology, 7(1), 49-55.

Gamella, J. F., Alvarez-Roldan, A., \& Romo, N. (1997). The content of ecstasy in Spain. Paper presented at the 7 th annual conference on drug use and drug policy, Amsterdam.

Gerra, G., Zaimovic, A., Giucastro, G., Maestri, D., Monica, C., Sartori, R., et al. (1998). Serotonergic function after (+/-)3,4-methylene-dioxymethamphetamine ('Ecstasy') in humans. International clinical psychopharmacology, 13(1), 1-9.

Green, A. R., O'Shea, E., \& Colado, M. I. (2004). A review of the mechanisms involved in the acute MDMA (ecstasy)-induced hyperthermic response. European journal of pharmacology, 500(1-3), 3-13.

Green, D. M., \& Swets, J. A. (1966). Signal detection theory and psychophysics. New York: Wiley. Hernandez Lopez, C., Farre, M., Roset, P. N., Menoyo, E., Pizarro, N., Ortuno, J., et al. (2002). 3,4-Methylenedioxymethamphetamine (ecstasy) and alcohol interactions in humans: psychomotor performance, subjective effects, and pharmacokinetics. $J$ Pharmacol Exp Ther.

Jentsch, J. D., \& Taylor, J. R. (1999). Impulsivity resulting from frontostriatal dysfunction in drug abuse: implications for the control of behavior by reward-related stimuli. Psychopharmacology, 146(4), 373-390.

Lamers, C. T., Ramaekers, J. G., Muntjewerff, N. D., Sikkema, K. L., Samyn, N., Read, N. L., et al. (2003). Dissociable effects of a single dose of ecstasy (MDMA) on psychomotor skills and attentional performance. Journal of psychopharmacology Oxford, England, 17(4), 379-387.

Linnoila, M., Virkkunen, M., George, T., \& Higley, D. (1993). Impulse control disorders. International clinical psychopharmacology, 8 Suppl 1, 53-56.

Linnoila, M., Virkkunen, M., Scheinin, M., Nuutila, A., Rimon, R., \& Goodwin, F. K. (1983). Low cerebrospinal fluid 5-hydroxyindoleacetic acid concentration differentiates impulsive from nonimpulsive violent behavior. Life sciences, 33(26), 2609-2614.

Logan, B. K., \& Couper, F. J. (2001). 3,4-Methylenedioxymethamphetamine (MDMA, ecstasy) and driving impairment. Journal of forensic sciences, 46(6), 1426-1433.

Logan, G. D. (1994). On the ability to inhibit thought and action. A users'guide to the stop signal paradigm. In D. D. T. H. Carr (Ed.), Inbibitory processes in attention, memory, and language (pp. 189-239). San Diego, California: Academic Press.

Logan, G. D., Cowan, W. B., \& Davis, K. A. (1984). On the ability to inhibit simple and choice reaction time responses: a model and a method. Journal of experimental psychology. Human perception and performance, 10(2), 276-291. 
McCann, U. D., Eligulashvili, V., Mertl, M., Murphy, D. L., \& Ricaurte, G. A. (1999).

Altered neuroendocrine and behavioral responses to $\mathrm{m}$-chlorophenylpiperazine in 3,4-methylenedioxymethamphetamine (MDMA) users. Psychopharmacology, 147(1), 56-65.

McCann, U. D., Ridenour, A., Shaham, Y., \& Ricaurte, G. A. (1994). Serotonin neurotoxicity after (+/-)3,4-methylenedioxymethamphetamine (MDMA; "Ecstasy"): a controlled study in humans. Neuropsychopharmacology official publication of the American College of Neuropsychopharmacology, 10(2), 129-138.

Monterosso, J., Ehrman, R., Napier, K. L., O'Brien, C. P., \& Childress, A. R. (2001). Three decision-making tasks in cocaine-dependent patients: do they measure the same construct? Addiction Abingdon, England, 96(12), 1825-1837.

Morgan, M. J. (1998). Recreational use of "ecstasy" (MDMA) is associated with elevated impulsivity. Neuropsychopharmacology official publication of the American College of Neuropsychopharmacology, 19(4), 252-264.

Morgan, M. J. (2000). Ecstasy (MDMA): a review of its possible persistent psychological effects. Psychopharmacology, 152(3), 230-248.

Mulvihill, L. E., Skilling, T. A., \& Vogel Sprott, M. (1997). Alcohol and the ability to inhibit behavior in men and women. Journal of studies on alcohol, 58(6), 600-605.

Nash, J. F. (1990). Ketanserin pretreatment attenuates MDMA-induced dopamine release in the striatum as measured by in vivo microdialysis. Life sciences, 47(26), 24012408.

Ortner, C. N. M., MacDonald, T. K., \& Olmstead, M. C. (2003). Alcohol intoxication reduces impulsivity in the delay-discounting paradigm. Alcohol and Alcoholism, 38(2), 151-156.

Parrott, A. C. (2000). Human research on MDMA (3,4-methylenedioxymethamphetamine) neurotoxicity: cognitive and behavioural indices of change. Neuropsychobiology, 42(1), 17-24.

Peroutka, S. J., Pascoe, N., \& Faull, K. F. (1987). Monoamine metabolites in the cerebralspinal fluid of recreational users of 3,4-methylenedioxymethamphetamine (MDMA; 'Ecstasy'). Research Communications in Substances of Abuse, 8(3-4), 125-138.

Reneman, L., Endert, E., de Bruin, K., Lavalaye, J., Feenstra, M. G., de Wolff, F. A., et al. (2002). The acute and chronic effects of MDMA ("ecstasy") on cortical 5-HT2A receptors in rat and human brain. Neuropsychopharmacology official publication of the American College of Neuropsychopharmacology, 26(3), 387-396.

Ricaurte, G. A., Finnegan, K. T., Irwin, I., \& Langston, J. W. (1990). Aminergic metabolites in cerebrospinal fluid of humans previously exposed to MDMA: preliminary 
observations. Annals of the New York Academy of Sciences, 600, 699-708; discussion 708-610.

Richards, J. B., Zhang, L., Mitchell, S. H., \& de Wit, H. (1999). Delay or probability discounting in a model of impulsive behavior: effect of alcohol. Journal of the experimental analysis of behavior, 71(2), 121-143.

Ridderinkhof, K. R., van den Wildenberg, W. P., Segalowitz, S. J., \& Carter, C. S. (2004). Neurocognitive mechanisms of cognitive control: the role of prefrontal cortex in action selection, response inhibition, performance monitoring, and reward-based learning. Brain and cognition, 56(2), 129-140.

Steele, T. D., McCann, U. D., \& Ricaurte, G. A. (1994). 3,4-

Methylenedioxymethamphetamine (MDMA, "Ecstasy"): pharmacology and toxicology in animals and humans. Addiction Abingdon, England, 89(5), 539-551.

Steele, T. D., Nichols, D. E., \& Yim, G. K. (1987). Stereochemical effects of 3,4methylenedioxymethamphetamine (MDMA) and related amphetamine derivatives on inhibition of uptake of. Biochemical pharmacology, 36(14), 2297-2303.

Swann, A. C., Bjork, J. M., Moeller, F. G., \& Dougherty, D. M. (2002). Two models of impulsivity: relationship to personality traits and psychopathology. Biological psychiatry, 51(12), 988-994.

Topp, L., Hando, J., Dillon, P., Roche, A., \& Solowij, N. (1999). Ecstasy use in Australia: patterns of use and associated harm. Drug and alcohol dependence, 55(1-2), 105-115.

Verkes, R. J., Gijsman, H. J., Pieters, M. S., Schoemaker, R. C., de Visser, S., Kuijpers, M., et al. (2001). Cognitive performance and serotonergic function in users of ecstasy. Psychopharmacology, 153(2), 196-202.

Volkow, N. D., Wang, G. J., \& Fowler, J. S. (1997). Imaging studies of cocaine in the human brain and studies of the cocaine addict. Annals of the New York. Academy of Sciences, 820, 41-54; discussion 54-45.

Volkow, N. D., Wang, G. J., Fowler, J. S., Logan, J., Gatley, S. J., Hitzemann, R., et al. (1997). Decreased striatal dopaminergic responsiveness in detoxified cocainedependent subjects. Nature, 386(6627), 830-833. 


\title{
CHAPTER 7
}

\section{ACUTE EFFECTS OF NOCTURNAL DOSES OF MDMA ON MEASURES OF IMPULSIVITY AND PSYCHOMOTOR PERFORMANCE THROUGHOUT THE NIGHT}

\author{
Kuypers, K.P.C., Wingen, M., Samyn, N., Limbert, N., and Ramaekers J.G.
}

Background. Previous studies on acute effects of MDMA on psychomotor performance and impulsivity showed that MDMA acts as a stimulant. These studies assessed performance during daytime whereas in real life, dance-attendees leaving a party use the drug during the night. Objective. The present study aimed to assess the effects of nocturnal doses of MDMA during the night and after a night of sleep deprivation, on psychomotor performance and impulsivity. Methods. Fourteen healthy subjects participated in a double-blind, placebo-controlled, twoway within-subject study. Treatments were MDMA 75 and $50 \mathrm{mg}$, administered at 8 PM and 12 PM respectively, or double placebo. Psychomotor and impulsivity tasks were conducted four times throughout the evening and night. A vigilance test was conducted once, at $5 \mathrm{AM}$ and a sleepiness scale was presented to the subjects 10 times throughout the evening and night. Results. MDMA impaired tracking performance in a simple tracking task. Divided attention task performance was also impaired as indicated by a decrease in secondary task performance under influence of MDMA, compared with placebo. MDMA did not affect impulsivity measures. Vigilance performance decreased as a function of time on task, but this decrement was less during MDMA treatment compared to placebo. After administration of MDMA, sleepiness scale scores were lower during the night when compared with placebo. This difference between MDMA and placebo disappeared in the morning. Conclusion. It is concluded that nocturnal doses of MDMA may produce impairments of tracking performance and divided attention throughout the night that are additive to performance impairment produced by sleep loss. 
112 | CHAPTER 7 


\section{Introduction}

Several acute studies have shown that MDMA acts as a psychomotor stimulant (Kuypers et al. 2006; Lamers et al. 2003; Ramaekers and Kuypers 2006; Ramaekers et al. 2006) (see also review (Dumont and Verkes 2006)). Lamers and colleagues (2003) demonstrated that a single dose of MDMA improved simple reaction time and tracking performance under single and dual task conditions. Ramaekers and Kuypers (2006) demonstrated that MDMA caused increased impulse control in a stop signal task, as shown by a decrease in stop reaction time. In addition, two actual driving studies showed improvement after a single dose of MDMA on automated driving skills as indicated by a reduction in weaving index (Kuypers et al. 2006; Ramaekers et al. 2006). When combined with alcohol however, MDMA's stimulant effects were not strong enough to overcome alcohol-induced impairment on psychomotor function and impulse control (Hernandez Lopez et al. 2002; Ramaekers and Kuypers 2006).

Studies showing stimulant effects of MDMA usually employed daytime dosing to subjects who had a normal night of sleep (Kuypers et al. 2006; Lamers et al. 2003; Ramaekers and Kuypers 2006; Ramaekers et al. 2006). Ecstasy users however usually take the drug in single or repeated doses during the evening or night, before going to a party or while partying (Farre et al. 2004; Hammersley 1999; ter Bogt and Engels 2005; Winstock et al. 2001). They subsequently stay awake the whole night while partying and dancing for hours on end (Parrott 2001).

The aim of the present study was to assess the acute effects of MDMA on psychomotor performance, impulsivity (behavioural inhibition and cognitive impulsivity) after repeated dosing during a night of sleep deprivation. The study was conducted according to a double-blind, placebo-controlled, within-subject design. Subjects received two doses of MDMA, separated by an interval of four hours, and were tested four times throughout the evening and night i.e. at 6.30 PM, $9.30 \mathrm{PM}, 1.30 \mathrm{AM}$ and $7 \mathrm{AM}$. The vigilance task was conducted only once at $5 \mathrm{AM}$.

\section{Methods}

\section{Design and treatments}

The study was conducted according to a double-blind, placebo-controlled, twoway within-subject design. The treatments consisted of two subsequent doses of MDMA (75 mg and $50 \mathrm{mg}$ ) and double placebo. According to the annual report (2005) of the 'Drugs information and monitoring system' (DIMS) of the 
Netherlands, average recreational doses of MDMA may vary between 0 and 200 $\mathrm{mg}$, taken as 1 or 2 pills per evening (DIMS 2005). Doses up to $150 \mathrm{mg}$ have previously been studied in acute studies. It was shown that side effects were limited with doses up to $125 \mathrm{mg}$ but appeared to be more frequent with $150 \mathrm{mg}$-doses (Cami et al. 2000; de la Torre et al. 2000). Dose administration was separated in time by 4 hours, at respectively 8 PM and 12 PM. A four-hour interval was chosen because subjects tend to take an additional dose when the subjective effects have worn off and that is approximately after 4 hours (Cami et al. 2000; Hammersley 1999). Placebo and MDMA were administered orally in identically appearing formulations. MDMA was administered as a $25 \mathrm{ml}$ solution in bitter orange peel syrup; placebo was given as $25 \mathrm{ml}$ bitter orange peel syrup, both were mixed with $200 \mathrm{ml}$ orange or apple juice.

\section{Participants}

Twenty volunteers underwent medical examination. Two volunteers were excluded from participation as they did not meet inclusion or exclusion criteria. The remaining subjects passed the medical screening and entered the training. Four subjects dropped-out prematurely either because of private matters $(3 \mathrm{x})$ or because of not feeling well on the first test day after placebo treatment $(1 \mathrm{x})$.

In total, fourteen subjects ( 7 males, 7 females) aged in the range of 19-33 years (mean (SD) 22.93 (3.75)) completed all study treatments. They all had used MDMA prior to onset of the study. Lifetime use of MDMA varied from light $(<25$ times) in 9 subjects to moderate (between 30-35 times) in five subjects.

Subjects were recruited by means of advertisements in local newspapers and the snowball technique. Potential candidates were questioned about their drug use and medical condition and were given information about the study. Thereupon they were sent a detailed brochure with information about the study procedure and two questionnaires for medical history and detailed history of drug use. Inclusion criteria were experience with MDMA use, free from psychotropic medication, good physical health, absence of major medical endocrine and neurological conditions, normal weight (Body Mass Index 18-28 kg/m²). Exclusion criteria were history of drug abuse (other than MDMA) or addiction, pregnancy or lactation, cardiovascular abnormalities (assessed by a standard 12-lead electrocardiogram (ECG)), excessive drinking ( $>20$ alcoholic consumptions), hypertension (diastolic $>100 \mathrm{mmHg}$; systolic $>170 \mathrm{mmHg}$ ), history of psychiatric or neurological disorders. A medical doctor checked the completed medical questionnaire, and upon approval, subjects were invited for a medical examination. Blood and urine 
samples were taken for examination and subjects underwent an ECG measurement. When medically fit, they were contacted and sent a brochure with information about the study and a number of rules they had to obey during the period of the study. Subjects signed an informed consent. They were paid for participating upon completion of the study.

The study was performed in accordance with the 1975 declaration of Helsinki, adjusted in Edinburgh (2000) and was approved by the Medical Ethics Committee of the Academic Hospital of Maastricht and the University of Maastricht. A permit for obtaining, storing and administering MDMA was obtained from the Dutch drug enforcement administration.

\section{Study procedure}

Before study onset, participants were familiarized with the tests on a training day. Participants were requested to abstain from any drug use 1 week before the medical examination until 14 days after the last test day. They were asked not to use any caffeinated or alcoholic beverages 24 hours before testing and to get a normal night's sleep. Participants were screened for alcohol use in breath and for recent drug use in urine (THC, opiates, cocaine, amphetamines, and methamphetamines) upon arrival at the laboratory test facilities at 6 PM. Women were given a pregnancy test. When positive, subjects were sent home. Subjects received a light meal and were given a sleep questionnaire. Cognitive tests were assessed four times during the evening and night i.e. at 6.30 PM (baseline measure), 9.30 PM, 1.30 AM and $7 \mathrm{AM}$; respectively 1.5 hours pre-dosing and, 1.5, 5.5 and 11 hours post-dosing ( $1^{\text {st }}$ dose). A sleep scale was presented to the subjects 10 times throughout the evening and night to assess their current level of sleepiness (Figure 1). The Mackworth clock task was conducted once, at 5 AM. A test day ended at 8.15 AM. A blood sample was collected before each test session, except at baseline, to determine MDMA and MDA concentrations in blood serum. Participants were kept awake the whole night (e.g. they played party games, watched TV and/or listened to music). Testing days were minimally separated by 7 days.

\begin{tabular}{|c|c|c|c|c|c|c|c|c|c|}
\hline Dose & & & $1^{\text {st }}$ & & $2^{\text {nd }}$ & & & & \\
\hline Time & $6: 00$ & $6: 30$ & $8: 00$ & $9: 30$ & 12:00 & $1: 30$ & 5:00 & 7:00 & $8: 15$ \\
\hline 1mine & PM & PM & PM & PM & PM & $\mathrm{AM}$ & $\mathrm{AM}$ & $\mathrm{AM}$ & $\mathrm{AM}$ \\
\hline Testblock & & $1^{\text {st }}$ & & $2^{\text {nd }}$ & & $3^{\text {rd }}$ & M & $4^{\text {th }}$ & End \\
\hline
\end{tabular}

Figure 1. Schematic representation of a test day. Test block contains all cognitive tasks described in article except Mackworth Clock Task (= M) 


\section{Cognitive assessments}

Critical tracking task. The critical tracking task (Jex et al. 1966) measures the subject's ability to control a displayed error signal in a 1st-order compensatory tracking task. Error appeared as horizontal deviation of a cursor from midpoint on a horizontal, linear scale. Subjects had to null the error by returning the cursor to the midpoint by means of compensatory joy-stick movements. The frequency of cursor deviations, and therefore its velocity, increased as a stochastic, linear function of time. The subject was required to make compensatory movements with a progressively higher frequency. Eventually his/her response frequency lagged the error signal by $180^{\circ}$ and control was lost. The frequency at which control loss occurs is commonly called the critical frequency or lambda-c $\left(?_{c}\right)$. The test was repeated five times and the median lambda-c over the five trials was used as the dependent variable.

Divided attention task. The divided attention task (Moskowitz 1973) assesses the ability to divide attention between two tasks performed simultaneously. The primary task is the same as the critical tracking task described above with the exception that the velocity of the error signal was kept constant at $50 \%$ of the subject's optimal performance on the training session $\left(?_{c} / 2\right)$. Tracking error was measured by the absolute distance $(\mathrm{mm})$ between the cursor's position and the center. The secondary task involved monitoring 24 single-digit numbers $(0-9)$ that were displayed in the four corners of the screen. The numbers changed asynchronously every 5 seconds. The requirement was to react as rapidly as possible by lifting their foot from a pedal any time a target, the numeral "2", appeared. Test duration was 12 minutes and dependent variables were tracking error for the primary task and reaction time of correct detections for the secondary task.

Stop-signal task. The current stop signal task is an adapted version of Fillmore and colleagues' task (2002) (Ramaekers and Kuypers 2006). The task requires subjects to make quick key responses to visually presented go-signals and to inhibit any response when a visual stop-signal is presented. The go signals were four $1.5 \mathrm{~cm}$ letters (A, B, C, and D) presented one at a time in the center of a computer screen. Subjects were required to respond to each letter as quickly as possible by pressing on one of two response buttons. One button was pressed to indicate that " $\mathrm{A}$ " or "C" appeared and the other to indicate "B" or " $\mathrm{D}$ " appeared. Letters were displayed for maximally 500 milliseconds and the computer screen was blank for 
1.5 seconds before the next letter was displayed. This provided a period of maximally 2 seconds in which the subject could respond to the displayed letter.

A single test consisted of 176 trials in which each of the 4 letter stimuli were presented equally often. A stop-signal occurred in 48 trials during the test. The stop-signal consisted of visual cue, i.e. “*”, that appeared in one of the four corners of the screen. Subjects were required to withhold any response in case a stop-signal was presented. Stop-signals were presented 12 times at each of the four delays i.e. 50, 150, 250 and 350 milliseconds after the onset of a letter, so in total on $27 \%$ of the trials.

Dependent variables were the percentage correct responses on go-trials, proportion of inhibitions on stop-trials and their respective reaction times i.e. GoRT and stopRT. Stop reaction time to stop-signal trials represented the estimated mean time required to inhibit a response.

Discounting task. The current discounting task was an adjusted version of Mitchell and colleagues' task (1999). It consisted of two subtasks, a delay and probability task. For each of the two tasks, subjects were presented 137 questions. For each question, subjects had to indicate which of two hypothetical rewards they preferred, the 'standard' or the 'alternative' item. In the delay task, the standard item $€ 10$, was available after one of five delays $(0,7,30,180$ or 365 days). In the probability task, the standard item $€ 10$ was available with one of five probabilities $(1.00,0.75,0.50,0.25$, or 0.10$)$. The alternative item was an amount of money $(€$ $0.01,0.25,0.50,1.00,1.50,2.00,2.50,3.00,3.50,4.50,4.50,5.00,5.50,6.00,6.50$, $7.00,7.50,7.50,8.00,8.50,8.50,9.00,9.50,10.00$, or 10.50) available after 0 days (delay task) or with a probability of 1.00 (probability task). During both subtasks, a standard and an alternative item were selected at random, without replacement, to form the question. That is, the alternative items were not presented in either a descending or ascending order for any standard item, and the standard items were not presented in a systematic order. Subjects expressed their preference for an item by pressing one of two buttons. Response choices were recorded and used afterwards to calculate the switch points at which the subject preferred the alternative item over the standard item. Task duration was 10 minutes.

Mackworth clock task. The Mackworth Clock task is a vigilance task which assesses sustained attention (Mackworth 1950; Ramaekers et al. 1995). Subjects were seated in front of a computer screen displaying a circular arrangement of 60 grey dots simulating the second mark on a clock. Dots were briefly illuminated in clockwise 
rotation at a rate of 0.5 seconds. Occasionally, the rotation proceeded with a 'double jump' (signal) by skipping one of the dots in the normal sequence. A total of 30 signals were presented during the whole task, so the target probability $(0.56 \%)$ was very low. Subjects had to detect the occurrence of these signals and respond to this by pressing a button. Within each period of fifteen minutes (= one block), ten signals were given. The test consisted of 3 blocks and test duration was 45 minutes. The dependent variable was the proportion of correct detections.

\section{Sleep assessment}

Stanford sleepiness scale. The Stanford sleepiness scale (Hoddes and et al. 1973) consists of 7 statements, describing stages of sleepiness ranging form feeling active and wide awake (1) to losing the struggle to remain awake and being nearly asleep (7). Subjects were asked every hour to choose the statement describing their current level of sleepiness.

\section{Pharmacokinetic assessments}

Blood samples were taken at $1.5,5.5$ and 11 hours post-dosing ( $1^{\text {st }}$ dose), centrifuged immediately at 4,000xg for $10 \mathrm{~min}$ and the corresponding serum samples were subsequently frozen at minus $20{ }^{\circ} \mathrm{C}$ until analysis for pharmacokinetic assessment. MDMA and MDA concentrations were determined using an integrated on-line SPE-LC-MS system (Symbiosis Pharma, Spark Holland) with tandem mass spectrometric detection (Quattro Premier, Water Corporation) using deuterated analogues for internal standardization. Quantification limits were $5.0 \mathrm{ng} / \mathrm{ml}$ for MDMA and $2.5 \mathrm{ng} / \mathrm{ml}$ for MDA.

\section{Statistical analyses}

All statistical analyses were conducted by means of the Statistical Package for the Social Sciences (SPSS version 11.5 for Windows). Paired t-tests were used to check for differences between baseline measures in the placebo test-session and the MDMA test-session. For performance measures, difference scores (score minus baseline) were calculated. These difference scores entered the general linear model (GLM) univariate repeated measures procedures with MDMA (2 levels) and Time (3 or 10 levels) as main within-subject factors. In the discounting task, an extra factor Delay (5 levels) for the delay discounting task, or Probability (5 levels) for the probability discounting task, was included. The Mackworth clock task was only conducted one time so factor time was here omitted from the analysis and factor Time on task (3 levels) was introduced. When there was no sphericity, F-tests were 
corrected by means of the Greenhouse-Geisser epsilon correction. The alpha criterion level of significance was set at $p=0.05$. In case of significant main effects of MDMA or Time, post-hoc analyses were conducted and alpha was then corrected for multiple comparisons.

\section{Results}

There were no statistically significant differences between baseline scores of the cognitive tasks or the sleep assessment. Nevertheless, it was also clear that some baseline differences existed that just failed to reach significance. In order to correct for this potential confounder it was decided to calculate difference scores from baseline for each performance measure.

\section{Cognitive assessments}

Critical tracking task. Analysis revealed significant main effects of MDMA $\left(\mathrm{F}_{1,13}=\right.$ 6.432; $\mathrm{p}=0.025)$ and Time $\left(\mathrm{F}_{2,26}=3.471 ; \mathrm{p}=0.046\right)$ on critical tracking (Figure 2). Further analysis of the MDMA effect showed that lambda scores were lower under the influence of MDMA compared with placebo. Contrast analysis (polynomial) of the Time effect revealed a quadratic relation between sessions $\left(\mathrm{F}_{1,13}=6.369\right.$; $\mathrm{p}=$ 0.025). There was no significant MDMA by Time interaction effect on critical tracking (Table 1).

Divided attention task. Primary task measures. ANOVA revealed significant effects of Time $\left(\mathrm{F}_{2,26}=15.737 ; \mathrm{p}<0.001\right)$ and MDMA by Time $\left(\mathrm{F}_{2,26}=5.403 ; \mathrm{p}=0.011\right)$ on Tracking Error (Figure 3). During placebo treatment, Tracking Error increased almost linearly as a function during the night. During MDMA treatment, tracking decreased during the night but returned to baseline levels early in the morning. Analysis revealed a significant effect of MDMA on number of Control Losses $\left(F_{1,13}=11.60 ; p=0.005\right)$. Under influence of MDMA, the number of Control Losses was larger compared with placebo. There was no significant effect of Time or MDMA by Time on number of Control Losses (Table 1).

Secondary task measures. There was a significant MDMA effect $\left(\mathrm{F}_{1,13}=9.969\right.$; $\mathrm{p}=0.008$ ) on Reaction Time (Figure 4). After administration of MDMA, subjects were slower in detecting targets compared to placebo. Analysis also revealed significant effects of MDMA $\left(\mathrm{F}_{1,13}=4.79 ; \mathrm{p}=0.047\right)$ and Time $\left(\mathrm{F}_{2,26}=4.66 ; \mathrm{p}=\right.$ 0.019) on number of Correct Detections. Under influence of MDMA, the number of Correct Detections was lower compared to placebo (Table 1). 


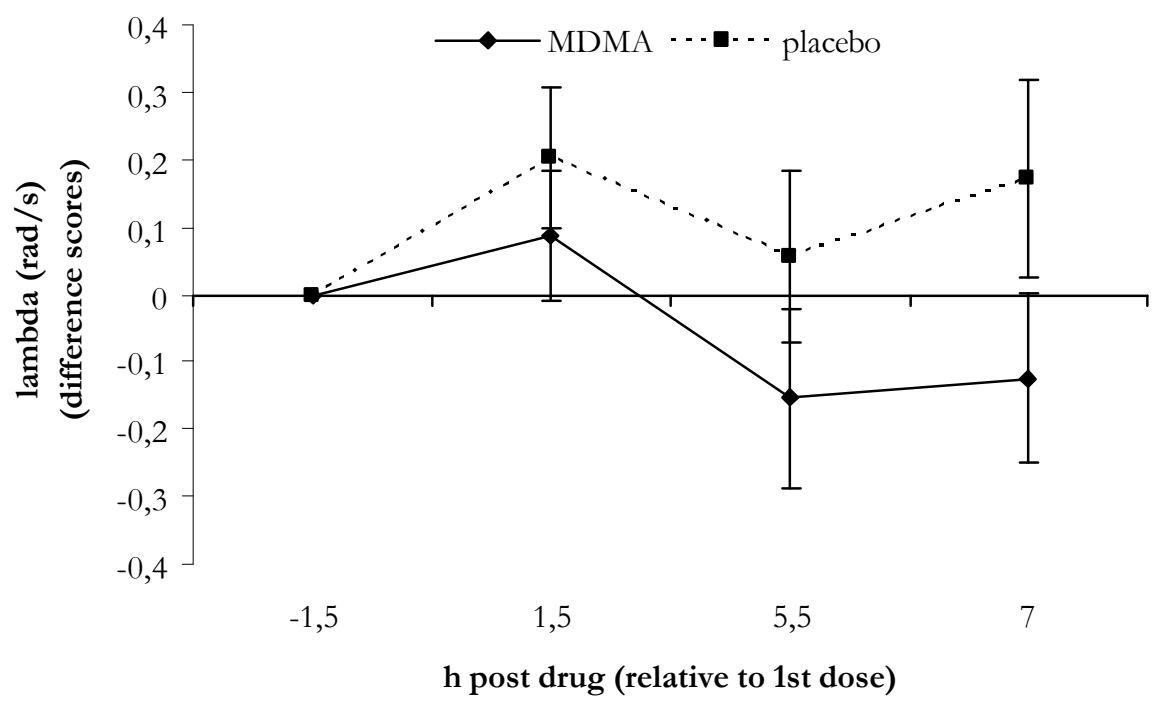

Figure 2. In this graph, critical tracking frequency in the Critical tracking task for both treatment conditions are shown as difference scores (relative changes to baseline).

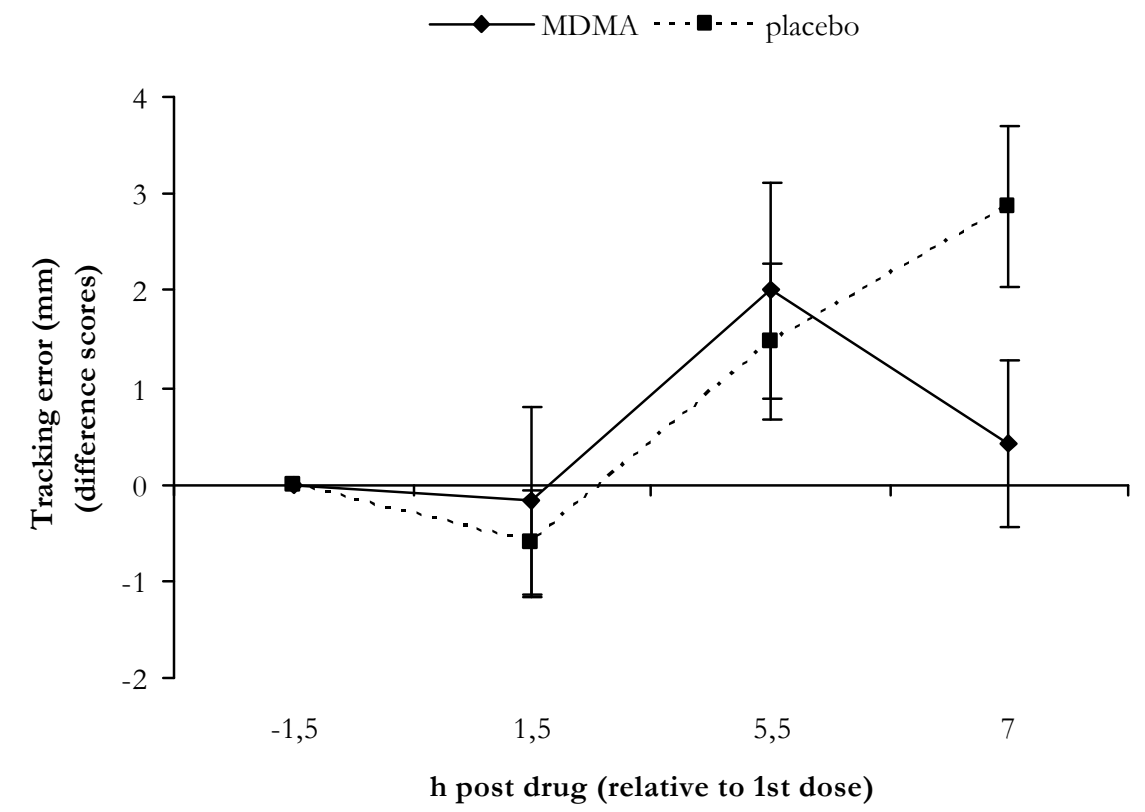

Figure 3. In this graph, mean Tracking Error (mm) (+SE) in the primary task of the Divided attention task for both treatment are shown as difference scores (relative changes to baseline). 
Stop-signal task. Response inbibition measures. There was no significant effect of MDMA, Time or MDMA by Time on Stop RT or proportion of Correct Inhibitions. Response execution measures. Analysis revealed a significant effect of Time $\left(F_{2,26}=4.65 ; \mathrm{p}=0.019\right)$ on the proportion of Correct Responses on the Go trials. There was no significant effect of MDMA, Time or MDMA by Time on Go RT (Table 1).

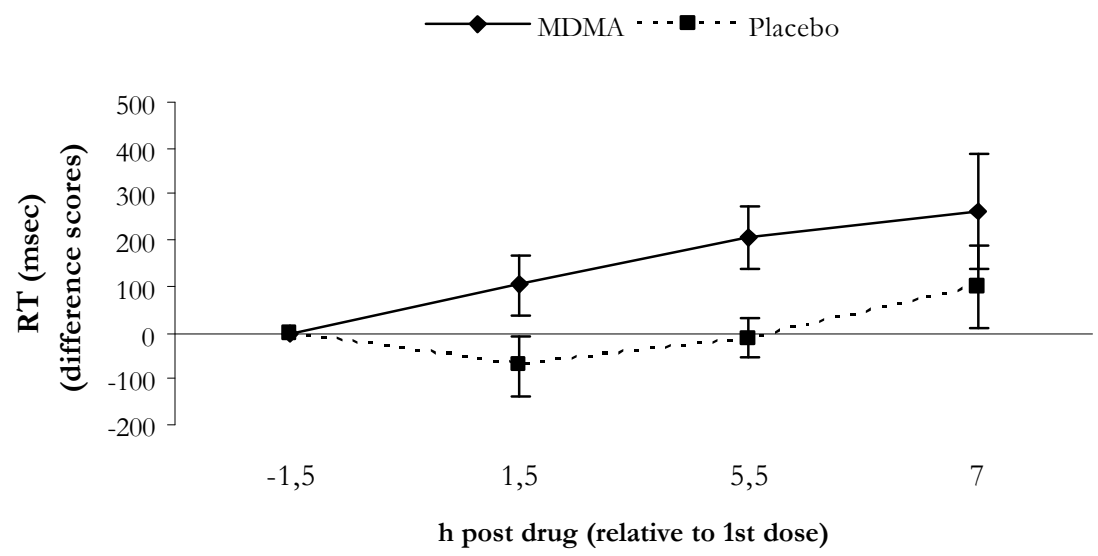

Figure 4. In this graph, mean reaction time (msec) (+SE) of Correct Detections in the secondary task of the Divided attention task for both treatment conditions are shown as difference scores (relative changes to baseline).

Discounting task. Two subjects displayed a random answer pattern on the discounting task and therefore their scores were omitted from the analysis. In both the subtasks, the switch point curves displayed a normal hyperbola-like pattern. The factor Delay or Probability was significant $\left(\mathrm{F}_{4,44}=5.154 ; \mathrm{p}=0.002\right)$ and $\left(\mathrm{F}_{4,44}=\right.$ 5.024; $\mathrm{p}=0.002)$ respectively for the delay and probability task. There were no significant effects of MDMA, Time or MDMA by Time on switch points in the delay or probability task (Table 1).

Mackworth clock task. Analysis revealed effects of Time on task (Block) $\left(\mathrm{F}_{2,26}=\right.$ 13.42; $\mathrm{p}<0.001)$ and Time on task by MDMA $\left(\mathrm{F}_{2,26}=5.19 ; \mathrm{p}=0.013\right)$ on proportion of Correct Detections. Vigilance performance decreased as a function of time on task, but this decrement was less during MDMA treatment as compared to placebo (Table 2). 
Table 1. Mean scores and SD's of dependent variables of the psychomotor and impulsivity tests, and pvalues of main and interaction effects on those variables.

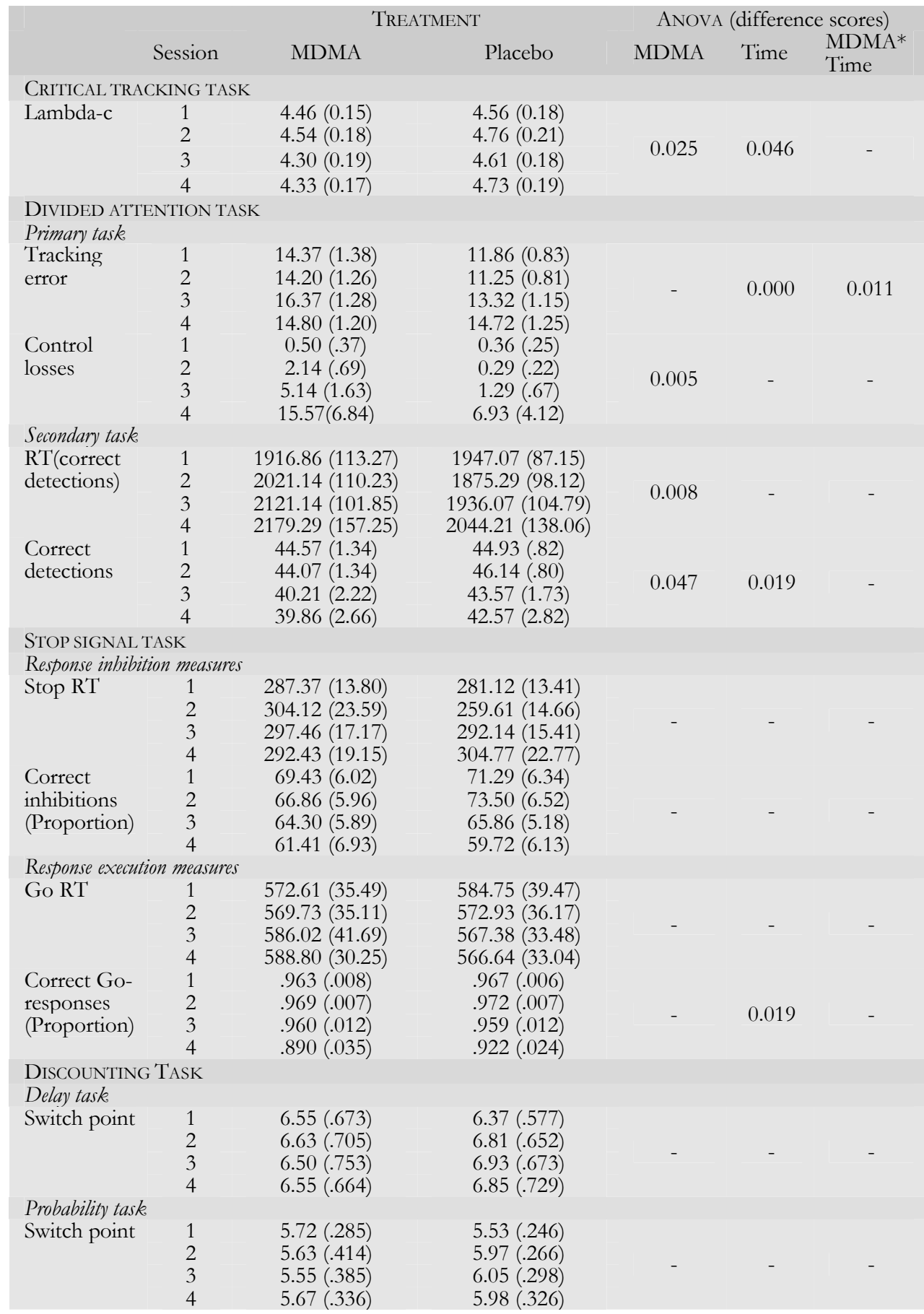


Table 2. Mean scores and SD's of the dependent variable (proportion of correct detections) of the Mackworth clock task, and p-values of main and interaction effects on that variable.

\begin{tabular}{|c|c|c|c|c|c|}
\hline \multirow[b]{2}{*}{ Block } & \multicolumn{2}{|c|}{ TREATMENT } & \multicolumn{3}{|c|}{ ANOVA } \\
\hline & MDMA & Placebo & MDMA & Block & MDMA*Blocl \\
\hline 1 & $.664(.089)$ & $.671(.056)$ & & & \\
\hline 2 & $.486(.080)$ & $.479(.070)$ & - & $<0.001$ & 0.013 \\
\hline 3 & $.536(.094)$ & $.336(.037)$ & & & \\
\hline
\end{tabular}

\section{Sleep assessment}

Analysis revealed significant effects of MDMA $\left(\mathrm{F}_{1,13}=5.892 ; \mathrm{p}=0.030\right)$, Time $\left(\mathrm{F}_{3.5 ; 45.6}=47.33 ; \mathrm{p}<0.001\right)$ and MDMA by Time $\left(\mathrm{F}_{9,117}=2.629 ; \mathrm{p}=0.008\right)$ on Sleepiness (Figure 5). Subjects progressively felt more fatigued. After administration of MDMA, subjects scored lower on the sleepiness scale compared with placebo. This difference between MDMA and placebo disappeared in the morning, when scores were equally high for both conditions.

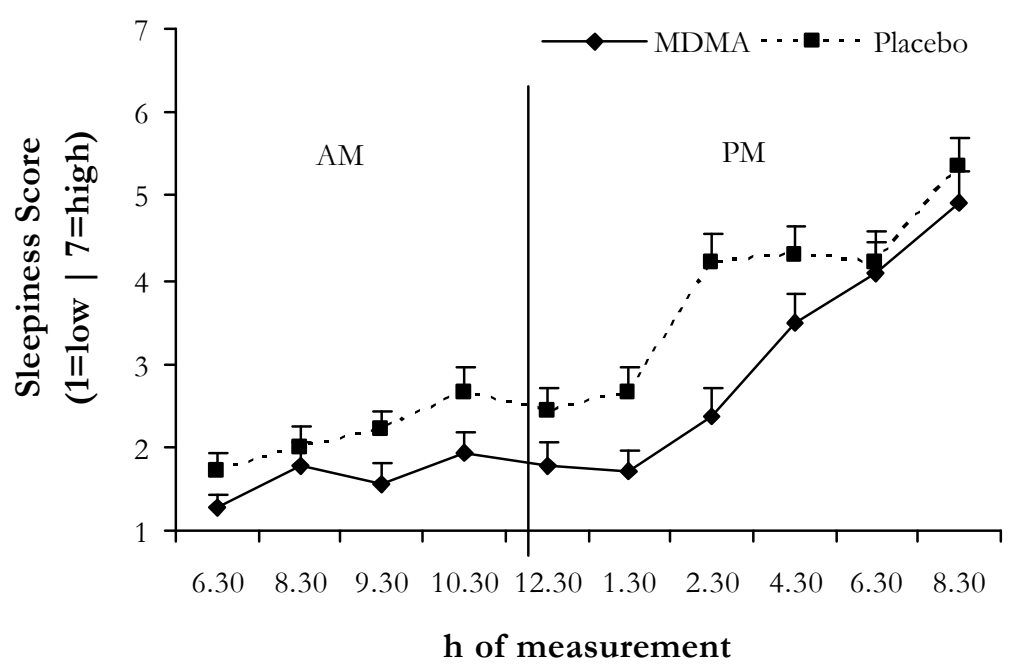

Figure 5. Mean scores (+SE) on the Stanford sleepiness scale as of function of hour of measurement.

\section{Pharmacokinetic assessments}

Mean (SD) blood serum concentrations of MDMA ( $\mathrm{ng} / \mathrm{ml})$ were 171.79 (54.63), 298.25 (65.38) and 199.27 (51.90), respectively 1.5, 5.5 and 11 hours post-dosing (1 $1^{\text {st }}$ dose). The higher concentrations in the second and third measurement are in agreement with the expected levels after administration of a $2^{\text {nd }}$ dose, 4 hours after the first dose. Mean (SD) concentrations of MDA were 6.35 (3.04), 17.84 (7.48), and 21.94 (9.17), respectively $1.5,5.5$ and 11 hours post-dosing ( $1^{\text {st }}$ dose). 


\section{Discussion}

Results showed that MDMA impaired performance in the Critical tracking task. MDMA also negatively affected Divided attention task performance as indicated by impairment of secondary task performance. Under influence of MDMA, subjects detected fewer targets and the corresponding reaction times were slower compared with placebo. This decrement in secondary task performance occurred in parallel with an MDMA-induced improvement of primary task performance. Primary task performance (i.e. tracking) sharply decreased during the night during both treatments. However, following MDMA, primary task performance also returned to baseline level in the morning. Measures of impulsivity, i.e. the Stopsignal task and the Discounting task were not affected by MDMA. The presence of impairment in the Critical tracking task and the Divided attention task as well as the absence of any MDMA effect on impulsivity tasks is in sharp contrast with previous studies who have demonstrated improvement of tracking performance and motor impulse control after a single, daytime dose of MDMA (Lamers et al. 2003; Ramaekers and Kuypers 2006). The main differences between these studies and the present study design are time of testing and time of drug administration. Previous studies have administered MDMA doses during the day and conducted performance testing during daytime. The present study employed nocturnal doses and assessed the effects of MDMA during the night and early in the morning after a night of sleep deprivation.

Collectively, these data thus seem to suggest that single doses of MDMA produce stimulant effects on psychomotor performance during the daytime but induce psychomotor impairment when taken in the evening or during the night. The mechanisms behind this differential effect of MDMA on performance are presently unknown but may be related to fluctuations in circadian rhythm and sleep loss during the night. Previous work has indicated that serotonin is important in the regulation of the circadian clock, which is located in the suprachiasmatic nuclei of the hypothalamus. Animal studies have shown that repeated exposure to MDMA can attenuate phase shifting in the body's circadian clock (Balogh et al. 2004; Colbron et al. 2002; Dafters and Biello 2003). It has been suggested that circadian rhythm disorders underlie the changes in sleep patterns and mood and mental performance changes that have often been reported in abstinent MDMA users (Colbron et al. 2002). In any case, performance impairment produced by MDMA was additive to the effect of sleep loss that caused a performance decrease throughout the night. After placebo treatment, performance in the Critical tracking 
task and the Divided attention task progressively decreased during the night due to sleepiness and sleep loss. MDMA did not counteract the effect of sleep loss but rather added more impairment. Additive impairment appeared to be constant during Critical tracking task performance and secondary task performance in the Divided attention test. It first appeared in the evening shortly after the $1^{\text {st }}$ dose of MDMA and remained stable until early in the morning, as evinced by the absence of an MDMA by Time after drug interaction.

Besides signs of impairment, there was also a clear indication of performance improvement during MDMA intoxication. Performance on the Mackworth clock task, a measure of sustained attention, deteriorated as function of time on task. This was evident from a progressive decrease in the proportion of correct detections that was observed during this 45-minutes task. This is basically a replication of the classical vigilance decrement that has repeatedly been shown under non-drug conditions in the same paradigm (Mackworth 1950; Teichner 1974). When under the influence of MDMA, subjects' performance deteriorated after the first fifteen minutes, but stayed relatively stable over the last 30 minutes of the task. This was not due to an increase in overall response rate as there was no significant effect on proportion of false alarms. It thus seems that MDMA mitigated the usual vigilance decrement which can be interpreted as stimulant activity. A similar impression emerged from subjective sleep recordings. These showed that subjects felt less sleepy during MDMA intoxication as compared to placebo. Many stimulant drugs have been shown to improve sustained attention during the day or during sleep loss (Bonnet et al. 2005; Koelega 1993), and the present vigilance date corroborate these findings.

It is concluded that nocturnal doses of MDMA may produce impairments of tracking performance and divided attention throughout the night that are additive to performance impairment produced by sleep loss.

\section{Acknowledgments}

We would like to thank. Nele Samyn, Gert De Boeck and Maria del Mar Ramirez Fernandez. from NICC, Brussels, for analyzing MDMA blood plasma samples and Nina Limbert for her belp with the data collection. 


\section{References}

Balogh B, Molnar E, Jakus R, Quate L, Olverman HJ, Kelly PA, Kantor S, Bagdy G (2004) Effects of a single dose of 3,4-methylenedioxymethamphetamine on circadian patterns, motor activity and sleep in drug-naive rats and rats previously exposed to MDMA. Psychopharmacology 173: 296-309

Bonnet MH, Balkin TJ, Dinges DF, Roehrs T, Rogers NL, Wesensten NJ (2005) The use of stimulants to modify performance during sleep loss: a review by the sleep deprivation and Stimulant Task Force of the American Academy of Sleep Medicine. Sleep 28: 1163-87

Cami J, Farre M, Mas M, Roset PN, Poudevida S, Mas A, San L, de la Torre R (2000) Human pharmacology of 3,4-methylenedioxymethamphetamine ("ecstasy"): psychomotor performance and subjective effects. Journal of clinical psychopharmacology 20: 455-66

Colbron S, Jones M, Biello SM (2002) MDMA alters the response of the circadian clock to a photic and non-photic stimulus. Brain research 956: 45-52

Dafters RI, Biello SM (2003) The effect of 3,4-methylenedioxymethamphetamine ('Ecstasy') on serotonergic regulation of the mammalian circadian clock mechanism in rats: the role of dopamine and hyperthermia. Neuroscience letters 350: 117-21

de la Torre R, Farre M, Ortuno J, Mas M, Brenneisen R, Roset PN, Segura J, Cami J (2000) Non-linear pharmacokinetics of MDMA ('ecstasy') in humans. British journal of clinical pharmacology 49: 104-9

DIMS (2005) DIMS Jaarbericht 2005. Trimbos institute Netherlands Institute of Mental health and Addiction, pp 1-4

Dumont GJ, Verkes RJ (2006) A review of acute effects of 3,4methylenedioxymethamphetamine in healthy volunteers. Journal of psychopharmacology Oxford, England 20: 176-87

Farre M, De La Torre R, O Mathuna B, Roset PN, Peiro AM, Torrens M, Ortuno J, Pujadas M, Cami J (2004) Repeated doses administration of MDMA in humans: pharmacological effects and pharmacokinetics. Psychopharmacology 173: 364-75

Hammersley R, Ditton, J., Smith, I., Short, E. (1999) Patterns of ecstasy use by drug users. British journal of criminology 39: 625-647

Hernandez Lopez C, Farre M, Roset PN, Menoyo E, Pizarro N, Ortuno J, Torrens M, Cami J, de La Torre R (2002) 3,4-Methylenedioxymethamphetamine (ecstasy) and alcohol interactions in humans: psychomotor performance, subjective effects, and pharmacokinetics. J Pharmacol Exp Ther

Hoddes E, et al. (1973) Quantification of sleepiness: A new approach. 
Jex HR, McDonnell JD, Phatak AV (1966) A "critical" tracking task for man-machine research related to the operator's effective delay time. I. Theory and experiments with a first-order divergent controlled element. NASA CR-616. NASA Contract Rep NASA CR: 1-105

Koelega HS (1993) Stimulant drugs and vigilance performance: a review.

Psychopharmacology 111: 1-16

Kuypers KPC, Samyn N, Ramaekers JG (2006) MDMA and alcohol effects, combined and alone, on objective and subjective measures of actual driving performance and psychomotor function. Psychopharmacology 187: 467-75

Lamers CT, Ramaekers JG, Muntjewerff ND, Sikkema KL, Samyn N, Read NL, Brookhuis KA, Riedel WJ (2003) Dissociable effects of a single dose of ecstasy (MDMA) on psychomotor skills and attentional performance. Journal of psychopharmacology Oxford, England 17: 379-87

Mackworth NH (1950) Researches on the measurement of human performance. (Med. Res. Council, Special Rep. Ser. No. 268.). Oxford, England: His Majesty"s Stationery Office, Oxford, England: His Majesty"s Stationery Office

Moskowitz H (1973) Laboratory studies of the effects of alcohol on some variables related to driving. Journal of Safety Research

Parrott AC (2001) Human psychopharmacology of Ecstasy (MDMA): A review of 15 years of empirical research. Human Psychopharmacology Clinical and Experimental 16: $557-577$

Ramaekers JG, Kuypers KPC (2006) Acute Effects of 3,4Methylenedioxymethamphetamine (MDMA) on Behavioral Measures of Impulsivity: Alone and in Combination with Alcohol. Neuropsychopharmacology official publication of the American College of Neuropsychopharmacology 31: 1048-55

Ramaekers JG, Kuypers KPC, Samyn N (2006) Stimulant effects of 3,4methylenedioxymethamphetamine (MDMA) $75 \mathrm{mg}$ and methylphenidate $20 \mathrm{mg}$ on actual driving during intoxication and withdrawal. Addiction DOI:10.1111/j.1360-0443.2006.01566.x: 1-8

Ramaekers JG, Muntjewerff ND, O'Hanlon JF (1995) A comparative study of acute and subchronic effects of dothiepin, fluoxetine and placebo on psychomotor and actual driving performance. British Journal of Pharmacology 39: 397-404

Teichner WH (1974) The detection of a simple visual signal as a function of time of watch. ter Bogt TFM, Engels RCME (2005) "Partying" hard: party style, motives for and effects of MDMA use at rave parties. Substance use and misuse 40: 1479-502 
128 | CHAPTER 7

Winstock AR, Griffiths P, Stewart D (2001) Drugs and the dance music scene: a survey of current drug use patterns among a sample of dance music enthusiasts in the UK. Drug and alcohol dependence 64: 9-17 


\title{
CHAPTER 8
}

\section{MDMA AND ALCOHOL EFFECTS, COMBINED AND ALONE, ON OBJECTIVE AND SUBJECTIVE MEASURES OF ACTUAL DRIVING PERFORMANCE AND PSYCHOMOTOR FUNCTION}

\author{
Kuypers, K. P. C., Samyn, N., and Ramaekers, J. G.
}

Background. The party drug ecstasy is frequently used in combination with other drugs like marihuana and alcohol. In addition, a substantial proportion of the MDMA users have claimed to drive a car when under the influence of MDMA and/or other drugs. Objective. To assess the effects of MDMA and alcohol, combined and alone, on actual driving performance and laboratory tasks related to driving. Methods. Eighteen healthy subjects participated in a double-blind, placebocontrolled, six-way cross-over study. Treatments consisted of MDMA 0, 75 and $100 \mathrm{mg}$ with and without alcohol; aiming at a blood alcohol concentrations of 0.06 $\mathrm{mg} / \mathrm{ml}$. Laboratory tests (Critical tracking task, Object movement estimation task) were conducted between 1.5 and 2 hours post-drug ( 0.5 and 1 hour post-alcohol). Actual driving tests (Road tracking test, Car-following test) were conducted between 3 and 5 hours post-drug ( 2 and 4 hours post-alcohol). Subjects completed the addiction research center inventory (ARCI) and rated their driving quality and mental effort during driving. Results. Alcohol alone impaired critical tracking performance, as well as a number of actual driving performance parameters (i.e standard deviation of lateral position (SDLP), brake reaction time, coherence). MDMA alone reduced SDLP and standard deviation of speed. MDMA significantly moderated alcohol induced impairment of road tracking performance but did not affect alcohol impairments of car-following and laboratory task performance. Subjective data seemed to support objective data. Conclusion. MDMA moderated the impairing effects of a low dose of alcohol on road tracking performance but it could not overcome alcohol-induced impairment on other aspects of driving behavior or driving-related performance.

Psychopharmacology, 2006; 187(4), 467-475 
130 | CHAPTER 8 


\section{Introduction}

The party drug ecstasy (3,4 methylenedioxymethamphetamine, MDMA) is frequently used in combination with other drugs (Barrett, Gross, Garand, \& Pihl, 2005; Logan \& Couper, 2001; Riley, James, Gregory, Dingle, \& Cadger, 2001; Topp, Hando, Dillon, Roche, \& Solowij, 1999). Attendees at raves or dance parties, have attested to the use of one $(2.7 \%)$ or more substances $(80 \%)$ during rave parties (Barrett et al., 2005). In half of the cases, MDMA was generally used in combination (92.3\%) with substances like cannabis (68.5\%), amphetamine (48.4\%) and/ or alcohol (45.2\%). Furthermore, when questioning rave attendees whether they would drive while using drugs, the conclusion was that up to $36 \%$ drove after having taken drugs (Riley et al., 2001). Several fatal and non-fatal road accidents have been reported in which MDMA was found in the plasma of drivers, alone or in combination with other drugs or alcohol (Carmen del Rio, Gomez, Sancho, \& Alvarez, 2002; Logan \& Couper, 2001). In addition, a few case reports have described erratic driving behaviors in drivers under the influence of MDMA. These behaviors included ignoring traffic signs and traffic lights and speeding (Henry, Jeffreys, \& Dawling, 1992; Schifano, 1995).

Experimental studies of the acute effects of MDMA on psychomotor performance and actual driving behavior have shown that certain aspects like tracking in a compensatory tracking task and weaving in an on-the-road driving task improved after a single dose whereas other aspects like time to contact estimation in a dual attention task, and gain, a parameter modeling speed adaptations, in an on-the-road car following task, deteriorated (Lamers et al., 2003; Ramaekers, Kuypers, \& Samyn, 2006). The improvement of certain aspects of actual driving performance seems to be mediated by manipulations of the dopaminergic system as similar driving improvement was noted for the dopaminergic agent methylphenidate. The impairment of more complex, controlled behavior as measured by the car-following task seems to be due to actions on the serotonergic system as methylphenidate did not affect this behavior whereas MDMA did (Ramaekers et al., 2006).

Up till now, only two studies investigated the effects of a MDMA-alcohol combination on performance, in a controlled experimental setting (Hernandez Lopez et al., 2002; Ramaekers \& Kuypers, 2006). Hernandez-Lopez and colleagues (2002) reported a discrepancy between subjective measures of sedation and objective measures of psychomotor impairment. MDMA moderated the subjective experience of alcohol-induced sedation; but it did not compensate for the alcohol- 
induced impairments on objective measures of psychomotor performance. Ramaekers and Kuypers (2006) found that increments in motor impulsivity caused by alcohol were not reduced by simultaneous administration of MDMA. Overall, these two studies seem to indicate that the stimulant effect of MDMA was not sufficient to compensate for the impairing effects of alcohol on psychomotor function and impulse control.

Brookhuis and colleagues (2004) conducted a quasi-controlled, naturalistic study to assess driving performance of MDMA users in an advanced driving simulator before and after visiting a rave party (Brookhuis, De Waard, \& Samyn, 2004). All subjects indicated that they had taken MDMA (average dose: 56mg) just before going to the party, and $30-40 \%$ of them also attested to the concomitant use of alcohol and/or marijuana. When retuning from the party, most of the subjects had taken additional doses of MDMA (70\%), marijuana (80\%) or alcohol (90\%). The participants were also tested sober, at a comparable time as the first MDMA ride. The results indicated that driving performance was not greatly affected prior to the rave party, but deteriorated during the night after multiple drug use. The most striking result was the apparent decreased sense for risk-taking as shown for example by the acceptance of smaller gaps between cars. This data suggest that combined use of MDMA and alcohol or marijuana produces more severe driving impairment than MDMA alone. However, it should be noted that these data are also confounded by time of testing. Driving impairment was primarily observed when subjects had build up a full-night of sleep loss, at the end of the rave. It can thus not be excluded that exhaustion by itself may also have played a major role in driver impairment observed after multiple drugs use.

The aim of the present study was primarily to assess the acute effects of a single dose of MDMA (75 mg or $100 \mathrm{mg}$ ) with and without a low dose of alcohol on actual driving performance in MDMA users in a double-blind, placebocontrolled, crossover design. Two psychomotor tasks were incorporated in the study to assess driving related behavior, i.e. tracking and time to contact. Two actual driving tests were included to assess different aspects of driving performance like automated behavior and driving at the tactical level, in the road tracking task and car following task respectively. Four subjective questionnaires were added to asses driving quality, mental effort and drug-induced effects. The primary question was whether the combined effects reduced or increased actual driving performance and driving-related behavior. 


\section{Methods}

\section{Design and treatments}

The study design was double-blind, placebo-controlled, six-way cross-over with balancing of the treatment orders. Orders were arranged according to three Latin squares and this resulted in 6 treatment orders which were randomly assigned to 18 subjects. The treatments consisted of MDMA 0,75 and $100 \mathrm{mg}$ with and without alcohol. MDMA and MDMA-placebo were administered orally in identically appearing formulations. MDMA was administered as a $25 \mathrm{ml}$-solution in bitter orange peel syrup, which was ingested at once. Alcohol dosing was designed to produce a peak blood alcohol concentration (BAC) of about $0.06 \mathrm{mg} / \mathrm{ml}$ during laboratory testing and a concentration that was under the legal limit for driving $(0.05 \mathrm{mg} / \mathrm{ml})$ during driving tests. Alcohol doses were administered at 45 minutes post-drug, and consumed within a period of 15 minutes. Before driving tests took place, subjects were given an additional dose of alcohol to keep their BAC levels approximately constant during the tests.

The doses of MDMA administered in this study were selected in the range of the commonly used doses that is 50 to $150 \mathrm{mg}$ (Henry, 1992; Parrott, 2004). Because the pharmacokinetics of MDMA appears to be non-linear, we selected 75 $\mathrm{mg}$ MDMA as our lowest dose and $100 \mathrm{mg}$ as our highest dose. A small increase in dose from $75 \mathrm{mg}$ to $100 \mathrm{mg}$ was expected to approximately double MDMA concentrations in blood (de la Torre et al. 2004).

Laboratory tests were conducted between 1.5 and 2 hours post-drug (0.5 and 1 hour post-alcohol) and driving tests took place between 3 and 5 hours post-drug ( 2 and 4 hours post-alcohol).

\section{Participants}

Eighteen recreational MDMA-users, 9 males and 9 females, were recruited through poster advertisements at Maastricht University and advertisements in local newspapers. Initial screening was based on a questionnaire on medical history and driving experience. Subjects who were accepted were examined by the medical supervisor, who also checked vitals signs and took blood and urine samples. Subjects underwent an electrocardiogram (ECG) measurement. Standard blood chemistry, haematological and drug screen tests were conducted on these samples. Inclusion criteria were: experience with the use of MDMA; free from psychotropic medication; good physical health as determined by examination and laboratory analysis; absence of any major medical, endocrine and neurological condition; normal weight, body mass index (weight/height ${ }^{2}$ ) between 18 and $28 \mathrm{~kg} / \mathrm{m}^{2}$; 
possession of a valid driving license and written Informed Consent. Exclusion criteria were: history of drug abuse (other than the use of MDMA) or addiction; pregnancy or lactation; cardiovascular abnormalities as assessed by standard 12lead ECG; excessive drinking (> 20 standard alcoholic consumptions a week); hypertension (diastolic $>100 \mathrm{mmHg}$; systolic $>170 \mathrm{mmHg}$ ); and history of psychiatric or neurological disorder.

Characteristics. Subjects had a mean (SD) age of 26.6 (5.4) and a mean (SD) weight of 70.39 (17.9). They drove on average (SD) 18444.44 (13536.3) km per year, and were all light-to-moderate users of MDMA who reported to have taken the drug on 2-25 occasions (mean: 9 occasions) in the previous year. Overall, subjects reported to have taken between 2-120 MDMA tablets (mean: 18 tablets) in the previous year. Seventy two percent of them admitted they had ever driven a car while intoxicated with MDMA of whom $83 \%$ had also done it in combination with alcohol.

This study was conducted according to the code of ethics on human experimentation established by the declaration of Helsinki (1964) and amended in Edinburgh (2000). All subjects gave their informed consent, in writing. Approval for the study was obtained from the University's Medical Ethics committee. A permit for obtaining, storing and administering MDMA was obtained from the Dutch drug enforcement administration. Subjects were paid for their participation in the study.

\section{Study procedure}

Subjects were asked to refrain from any drugs starting 1 week before the medical screening and physical examination until two weeks after the last experimental session. The subjects were not allowed to use alcohol or caffeinated beverages on the day prior to an experimental session and were requested to arrive at experimental sessions well rested. Drug screens in urine and alcohol screens in breath were performed prior to experimental sessions upon arrival of the subject. Subjects who tested positive on alcohol or drugs screens were sent home and scheduled to return to the laboratory at a later time. Subjects were transported from their homes to the laboratory or vice versa by one of the experimenters in order to prevent subjects from driving under the influence on the day of testing. Additional clinical blood chemistry, with particular reference to liver and renal function was conducted at day 7 after each treatment. All subjects received a training session prior to onset of the experimental sessions in order to familiarize them with the tests and procedures. The minimum wash-out period between successive treatments was one week. 


\section{Psychomotor assessments}

Critical tracking task. The critical tracking task (CTT) (Jex, McDonnell, \& Phatak, 1966) measures the subject's ability to control a displayed error signal in a compensatory tracking task. Error appears as horizontal deviation of a cursor from midpoint on a horizontal, linear scale. Compensatory joy-stick movements null the error by returning the cursor to the midpoint. The frequency of cursor deviations, and therefore its velocity, increases as a stochastic, linear function of time. The subject is required to make compensatory movements with a progressively higher frequency. Eventually his response frequency lags the error signal in a way that control is lost. The frequency at which control loss occurs is commonly called lambda-c (?c) or the critical frequency. The subject performs this test in five trials on each occasion and the mean ? is recorded as the final score.

Object movement estimation under divided attention. The object movement estimation under divided attention task (OMEDA) (Read, Ward, \& Parkes, 2000) assesses the subject's ability to estimate of speed of movement, and time to contact (TTC) of a moving object to a fixed point under divided attention. The subject is seated in front of a computer screen of which the corners are covered by green triangles and a yellow circle occludes the centre of the screen. The occlusion circle varies in size per trial (2, 100 or 200 pixels). From one of the corners, a red dot (target) travels towards the centre of the screen. Once it encounters the edge of the occlusion circle, it travels underneath and is no longer visible. The subject has to indicate at what point in time the target reaches the centre of the computer screen, by pressing a foot pedal; this is the primary task. During movement of the target 5 geometrical shapes appear; one on top of the occlusion circle and one on top of each of the triangles in the corners. For the secondary task the subject is required to press a button in case the geometric shape at the occlusion circle matches one of the others. Absolute TTC error -defined as the absolute mean difference between estimated and actual TTC-, and the number of correct responses to the geometric targets, are the primary and secondary measures. The test was originally designed as an off-road driving capacity assessment task.

\section{Actual driving tests}

Road tracking test. In the road tracking test (O'Hanlon, 1984) the subject operates a specially instrumented vehicle over a $100 \mathrm{~km}$ primary highway circuit while maintaining a constant speed $(95 \mathrm{~km} / \mathrm{h})$ and a steady lateral position between the delineated boundaries of the right (slower) traffic lane. An electro-optical device 
mounted at the rear back of the car continuously measures lateral distance separating the vehicle and the left lane-line. This signal is stored on an onboard computer disk for later off-line editing wherein all data segments that reveal signal loss, disturbance or occurrence of passing manoeuvres are removed. The remaining data are then used to calculate means and variances for lateral position and speed (SP). Standard deviation of lateral position (SDLP), a measure of road tracking error, is taken as the primary outcome variable. Duration of the test is 1 hour.

Car-following test. The car-following test (Brookhuis, de Waard, \& Mulder, 1994; Ramaekers, Robbe, \& O'Hanlon, 2000) involves the use of two vehicles traveling in tandem at speeds of around $70 \mathrm{~km} / \mathrm{h}$ on a secondary highway. The preceding vehicle is under the control of an investigator. The following vehicle is under the control of the subject who is accompanied by a licensed driving instructor, like in the former test. Subjects attempt to drive $15-30 \mathrm{~m}$ behind the preceding vehicle and to maintain that headway as it executes a series of approximately 6 deceleration maneuvers (sinusoidal speed changes). Between deceleration maneuvers, the investigator in the leading car randomly activates the brake lights of his vehicle while the speed of the leading car remains constant at $70 \mathrm{~km} / \mathrm{h}$. The subject is instructed to react to brake lights by removing his/her foot from the speed pedal as fast as possible. This procedure is repeated between 20 and 30 times throughout the test. Dependent variables are brake reaction time (BRT, in $\mathrm{msec}$ ), and three parameters derived from the analysis of coherence between the speeds of the leading and following car, i.e. time to speed adaptation (TSA, msec), coherence and gain. Time to speed adaptation, also known as 'phase shift', is an estimation for how long it takes the subject to react to speed adaptations of the leading car. The coherence parameter reflects the accuracy of the speed adaptation of the subject. This squared correlation can range from 0 , when there is no coherence, to 1 , which means that there is perfect coherence. Gain or modulus is an amplification factor between the signals of the two cars. This will be larger than 1 when the subject overreacts to speed adaptations of the leading car. Duration of the test is 25 minutes.

\section{Subjective measures}

Two 10-centimeter visual analogue driving quality scales, were rated by the subjects at the start (2.5 hours post-drug) and the end (4.5 hours post-drug) of driving tests, to assess their prediction and evaluation of their driving quality. In addition, the subjects filled out a mental effort scale (Zijlstra, 1993) and the addiction research 
center inventory (ARCI) scale after completing the driving tests. The mental effort scale, a visual analogue scale of $15 \mathrm{~cm}$, assessed the extent to which the subjects experienced the driving tests as mentally demanding. The ARCI contained 49true-false items, all sensitive to the subjective effects of several classes of abused drugs. Items were clustered into five scales: Morphine-Benzedrine Group (MBG), Amphetamine (A), Benzedrine Group (BG), Pentobarbital-Chlorpromazine (PCAG) and Lysergic Acid (LSD), and they measured respectively drug-induced euphoria, stimulant-like effects, intellectual efficiency and energy, sedation, and dysphoria (de Wit, Crean, \& Richards, 2000; Haertzen, 1965).

\section{Pharmacokinetic assessments}

Blood samples were taken at 1.5 and 5.5 hours post-drug, centrifuged immediately and subsequently frozen at minus $20^{\circ} \mathrm{C}$ until analysis for pharmacokinetic assessment. MDMA and MDA concentrations were determined using solid phase extraction and gas chromatography with mass spectrometric detection with quantification limits of $1 \mathrm{ng} / \mathrm{ml}$.

Blood alcohol concentrations were assessed every 15 minutes during the first 2 hours after drinking and at the start and end of driving tests, with a Lion SD-4 Breath Alcohol Analyzer.

\section{Statistical analyses}

All statistical analyses were conducted by means of SPSS 11.5 for Windows. Each variable was analyzed using GLM univariate repeated measures procedures with Alcohol (2 levels) and MDMA (3 levels) as main within-subject factors. Separate drug-placebo contrasts were conducted following an overall effect of MDMA or the interaction between MDMA and Alcohol. The alpha criterion level of significance was set at $p=0.05$.

\section{Results}

\section{Missing values}

Due to technical dysfunctions no data were collected in the CT'T and the OMEDA on a single occasion. These missing values were replaced in the statistical analysis by the means of the other 17 subjects in that particular treatment condition. There were no missing data in both driving tests.

\section{Failures to complete the driving tests}

Four driving tests were terminated before scheduled completion because the driving instructor felt the subjects were unable to continue. These failures to 
complete the driving tests occurred in different subjects, during treatment with alcohol alone (2 times), MDMA 75 and alcohol combined (1 time) and placebo (1 time). All data from all driving tests entered the statistical analysis.

\section{Psychomotor assessments}

Critical tracking task. Analysis revealed a significant main effect of Alcohol $\left(\mathrm{F}_{1,17}=\right.$ 17.53; $\mathrm{p}=0.001)$ on critical tracking $\left(\mathrm{e}_{\mathrm{c}}\right)$. Alcohol impaired tracking performance as shown by a decrease in ? in alcohol conditions (mean (SE)) (3.82(0.15)) compared to the non-alcohol conditions $(4.19(0.12))$. There were no significant effects of MDMA or MDMA by Alcohol (Fig.1; Table 1).

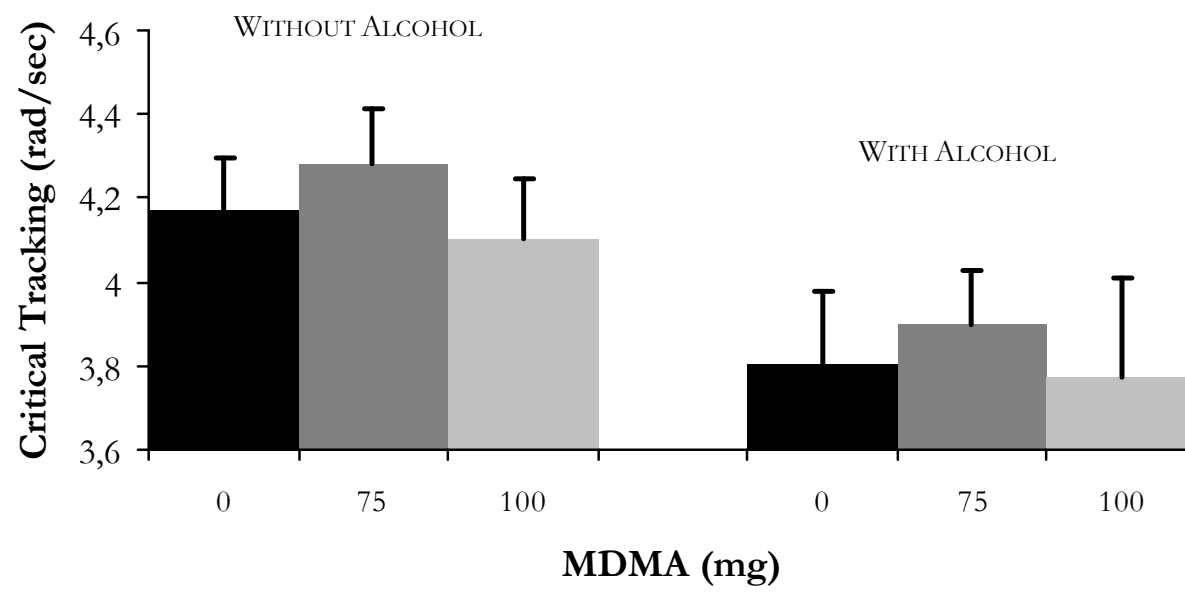

Figure 1. Mean (SE) critical tracking (rad/sec) in the CTT during every treatment condition.

Object movement estimation under divided attention. There were no significant main effects of Alcohol, MDMA or MDMA by Alcohol on the TTC error (Table 1).

\section{Actual driving tests}

Road tracking test. Analysis revealed significant main effects on SDLP of Alcohol $\left(\mathrm{F}_{1,17}=33.37 ; \mathrm{p}<0.001\right)$, MDMA $\left(\mathrm{F}_{2,34}=12.12 ; \mathrm{p}<0.001\right)$, and MDMA by Alcohol $\left(\mathrm{F}_{2,34}=3.27 ; \mathrm{p}=0.05\right)$. SDLP increased by $2.5 \mathrm{~cm}$ under the influence of alcohol, and decreased by approximately $2 \mathrm{~cm}$ after both doses of MDMA, compared with placebo. Inspection of the MDMA-Alcohol interaction data showed that the stimulating effect of MDMA $100 \mathrm{mg}$ on SDLP was more prominent when combined with alcohol whereas the stimulating effect of MDMA $75 \mathrm{mg}$ did not change in magnitude after alcohol co-administration (Figure 2, Table 1). 

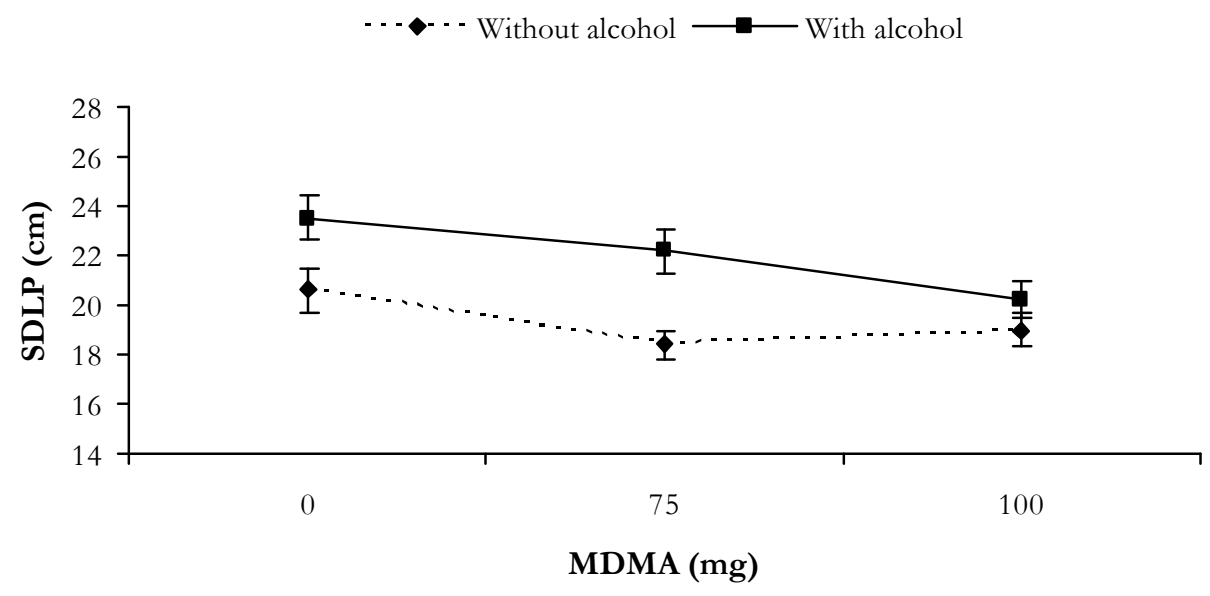

Figure 2. Mean (SE) Standard deviation of lateral position (SDLP, cm) in the Road tracking test during every treatment condition.

Analysis also showed a significant main effect of MDMA $\left(\mathrm{F}_{2,34}=5.38 ; \mathrm{p}=0.009\right)$ on SD Speed. Contrast analysis revealed this effect was due to both the MDMA 75 condition $\left(\mathrm{F}_{1,17}=8.20 ; \mathrm{p}=0.011\right)$ and MDMA 100 condition $\left(\mathrm{F}_{1,17}=7.44 ; \mathrm{p}=\right.$ 0.014) compared with placebo. Both doses of MDMA significantly decreased SDSP by about $0.2 \mathrm{~km} / \mathrm{h}$ relative to placebo. There were no effects of Alcohol, MDMA or MDMA by Alcohol on Lateral Position (LP) or Speed (Table 1).

Car-following test. Analysis revealed significant main effects of Alcohol on BRT $\left(\mathrm{F}_{1,17}=5.76 ; \mathrm{p}=0.028\right)$ and Coherence $\left(\mathrm{F}_{1,17}=7.44 ; \mathrm{p}=0.014\right)$. On the average, alcohol increased Brake reaction time by 40 milliseconds. Additional drug-placebo comparisons revealed that both MDMA by alcohol conditions did not differ significantly from placebo (Fig. 3; Table 1).

There were no significant main effects of Alcohol, MDMA or MDMA by Alcohol on Gain or TSA (Table 1). 
Table 1. Mean (SE) values of laboratory measures of psychomotor performance and parameters of the actual driving tests and p-values of main and interaction effects on those variables.

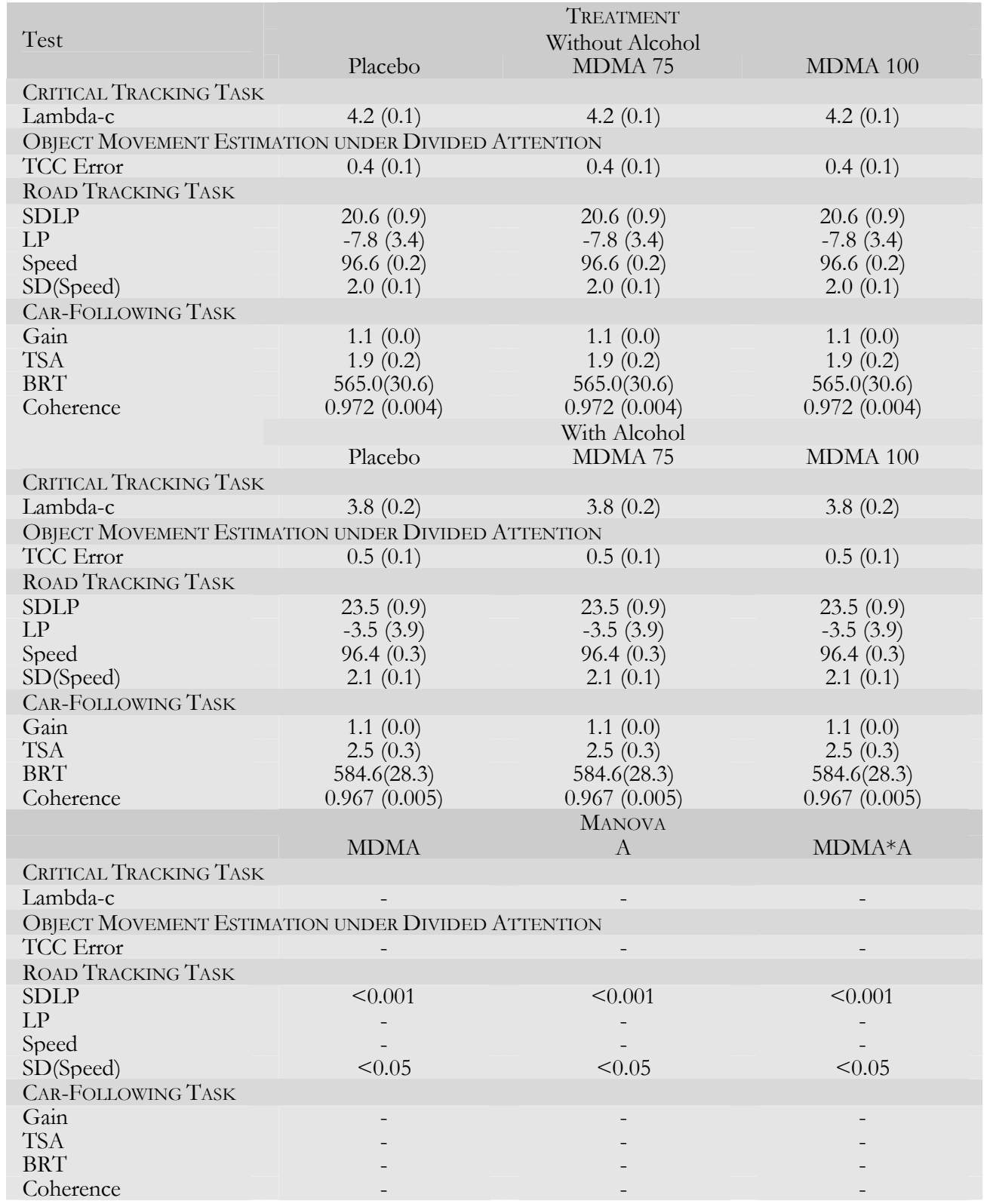




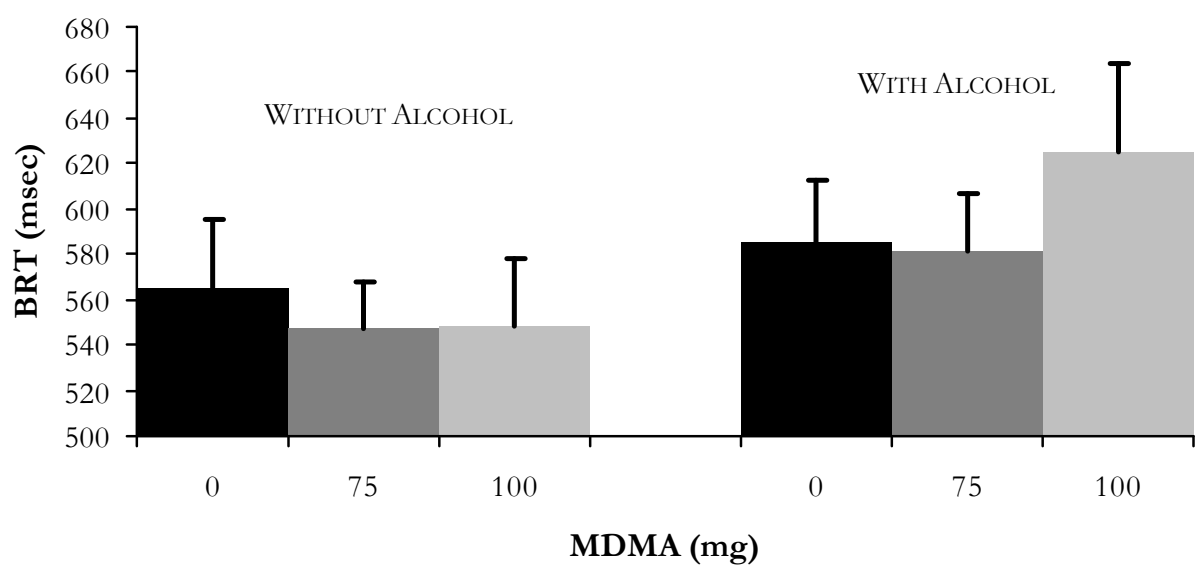

Figure 3. Mean (SE) Brake reaction time (BRT, msec) in the Car-following-test during every treatment condition.

\section{Subjective assessments}

Prior to the driving test, subjects predicted a reduction in their driving ability during treatment with Alcohol $\left(\mathrm{F}_{1,17}=16.55 ; \mathrm{p}=0.001\right)$ and MDMA $\left(\mathrm{F}_{1.47,24.92}=\right.$ 19.86; $\mathrm{p}<0.001)$. At completion of the driving test, only MDMA was rated to positively affect their driving ability $\left(\mathrm{F}_{2,34}=11.19 ; \mathrm{p}<0.001\right)$. There was no significant interaction effect of MDMA by Alcohol. Mental effort during driving significantly increased during Alcohol treatment $\left(\mathrm{F}_{1,17}=6.28 ; \mathrm{p}=0.023\right)$ but decreased after MDMA $\left(\mathrm{F}_{1.49 ; 25.35}=6.18 ; \mathrm{p}=0.011\right)$. Further analysis of the MDMA effect showed this effect was caused by the MDMA 100 condition $(p=0.04)$; MDMA 75 failed to reach significance $(\mathrm{p}=0.058)$. Mental effort was not affected by the MDMA-Alcohol combination (Table 2).

Table 2. Mean (SE) values of subjective evaluations of driving quality, mental effort and p-values of main and interaction effects.. $D Q=$ Driving quality; treatment: $M=M D M A, A=$ Alcohol.

\begin{tabular}{|c|c|c|c|c|c|c|c|c|c|}
\hline \multirow[b]{3}{*}{ Test } & \multicolumn{6}{|c|}{ TREATMENT } & \multirow{2}{*}{\multicolumn{3}{|c|}{ AnOva }} \\
\hline & \multicolumn{3}{|c|}{ Without Alcohol } & \multicolumn{3}{|c|}{ With Alcohol } & & & \\
\hline & PLA & M75 & M100 & PLA & M75 & M100 & M & A & $\mathrm{M}^{*} \mathrm{~A}$ \\
\hline Predicted & .53 & 2.49 & 2.58 & 1.33 & 4.66 & 4.08 & $<00001$ & 0.001 & 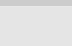 \\
\hline DQ & $(.25)$ & $(.60)$ & $(.56)$ & $(.45)$ & $(.49)$ & $(.67)$ & -0.001 & 0.001 & - \\
\hline Evaluated & 3.63 & 2.35 & 2.26 & 5.12 & 3.60 & 2.08 & $<0 \Omega 01$ & & 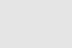 \\
\hline DQ & $(.65)$ & $(.50)$ & (.38) & $(.65)$ & $(.56)$ & $(.26)$ & $<0.001$ & - & - \\
\hline Mental & 4.58 & 3.92 & 3.10 & 6.89 & 4.19 & 3.71 & 01 & 23 & \\
\hline Effort & $(0.89)$ & $(0.54)$ & $(0.48)$ & $(0.78)$ & $(0.66)$ & $(0.53)$ & 0.011 & 0.025 & - \\
\hline
\end{tabular}


ANOVA also revealed significant main effects of MDMA on two scales of the ARCI: i.e., $\mathrm{MBG}\left(\mathrm{F}_{2,34}=14.78 ; \mathrm{p}=0.000\right)$ and $\mathrm{A}\left(\mathrm{F}_{2,34}=6.31 ; \mathrm{p}=0.005\right)$. Subjects felt more euphoric and experienced stimulant-like effects when they received MDMA (both doses), compared with placebo. Furthermore, there were also two significant interaction effects of MDMA by Alcohol on PCAG $\left(\mathrm{F}_{2,34}=4.92\right.$; $\mathrm{p}=$ $0.013)$ and $B G\left(F_{2,34}=5.32 ; p=0.010\right)$. These interactions indicated that alcohol produced sedation and lethargy when given alone, and that combined use with MDMA counteracted feelings of sedation and lethargy (Table 3).

Table 3. Mean (SE) values of 'drug-induced states' (ARCI Scales: MBG: Morphine-Benzedrine Group (drug-induced euphoria), A: Amphetamine (stimulant-like effects), BG: Benzedrine Group (intellectual efficiency and energy), PCAG: Pentobarbital-Chlorpromazine (sedation), and LSD: Lysergic Acid (dysphoria): treatments: $M=M D M A ; A=$ Alcohol

\begin{tabular}{lccccccccc}
\multicolumn{8}{c}{ Without alcohol } & \multicolumn{9}{c}{ TREATMENT } & \multicolumn{3}{c}{ With alcohol } & & & \\
SCALE & PLA & M75 & M100 & PLA & M75 & M100 & M & A & M*A \\
MBG & 1.83 & 3.50 & 4.11 & 1.67 & 4.94 & 5.22 & $<0.001$ & - & - \\
& $(0.29)$ & $(0.71)$ & $(0.85)$ & $(0.59)$ & $(1.07)$ & $(0.96)$ & & & \\
A & 1.11 & 2.00 & 2.28 & 1.06 & 2.94 & 2.67 & 0.005 & - & - \\
& $(0.21)$ & $(0.48)$ & $(0.51)$ & $(0.21)$ & $(0.68)$ & $(0.54)$ & & & \\
BG & 1.22 & 1.22 & 1.5 & -0.22 & 1.72 & 1.28 & & - & 0.010 \\
& $(0.31)$ & $(0.56)$ & $(0.60)$ & $(0.45)$ & $(0.55)$ & $(0.45)$ & - & & \\
PCAG & -0.11 & 0.28 & -0.06 & 3.17 & -0.06 & 0.11 & & & 0.013 \\
& $(0.57)$ & $(0.82)$ & $(0.75)$ & $(0.93)$ & $(0.70)$ & $(0.78)$ & - & - & \\
LSD & -1.50 & -1.06 & -0.72 & -1.17 & -0.94 & -0.89 & & & - \\
& $(0.18)$ & $(0.30)$ & $(0.31)$ & $(0.33)$ & $(0.27)$ & $(0.30)$ & - & - & -
\end{tabular}

\section{Pharmacokinetics}

Mean Blood Alcohol Concentrations (BAC) did not differ significantly between treatments. Mean BACs during performance in the Road tracking test were respectively $.37, .41$ and $.42 \mathrm{mg} / \mathrm{ml}$ following placebo, MDMA $75 \mathrm{mg}$ and MDMA $100 \mathrm{mg}$. During car-following, mean BACs varied from .38, .43 and .41 mg/ml respectively following placebo, MDMA $75 \mathrm{mg}$ and MDMA $100 \mathrm{mg}$. Descriptive parameters of BAC, MDMA and its main metabolite MDA are given in tables 4 and 5.

Table 4. Mean (SD) Blood Alcohol Concentrations as a function of time post dosing in alcohol related treatment conditions $(N=18) ; A=$ Alcohol; $R T$ test $=$ Road Tracking test

$\begin{array}{cccccc}\begin{array}{c}\text { Time post } \\ \text { drinking }(\mathrm{h})\end{array} & \begin{array}{c}\text { Time post } \\ \text { drug }(\mathrm{h})\end{array} & \text { Activity } & \text { A } & \begin{array}{c}\text { TrEATMENT } \\ \text { MDMA75+A }\end{array} & \text { MDMA100+A } \\ 0.5 & 1.5 & \text { Onset labtests } & .65(.19) & .56(.12) & .57(.13) \\ 1.25 & 2.25 & \text { End labtests } & .56(.16) & .56(.08) & .58(.10) \\ 2 \text { or } 3.5 & 3 & \text { Onset CF test } & .38(.02) & .43(.03) & .41(.02) \\ 2.5 & 3.5 & \text { Onset RT test } & .37(.02) & .41(.02) & .42(.02) \\ 4 & 5 & \text { End Driving tests } & .29(.10) & .36(.07) & .34(.11)\end{array}$


Table 5. Mean (SD) concentrations $(\mathrm{ng} / \mathrm{ml})$ of MDMA and $M D A$ at 1.5 and 5.5 hours post-dosing in every treatment condition $(N=18) ; L T=$ Laboratory tests; $D T=$ Driving tests.

$\begin{array}{lllcccc}\begin{array}{c}\text { Time post- } \\ \text { drug (h) }\end{array} & \text { Activity } & & \text { MDMA75 } & \text { MDMA100 } & \text { MDMA75+A } & \text { MDMA100+A } \\ 1.5 & \text { Onset } & \text { MDMA } & 137.4(31.9) & 191.8(49.1) & 147.1(36.3) & 208.5(45.7) \\ & \text { LT } & \text { MDA } & 3.5(1.2) & 4.5(1.5) & 3.4(1.0) & 4.7(1.4) \\ 5.5 & \text { End DT } & \text { MDMA } & 113.5(31.2) & 163.0(40.7) & 107.9(25.4) & 152.6(40.8) \\ & & \text { MDA } & 6.8(1.7) & 9.0(1.9) & 6.5(1.5) & 8.8(2.1)\end{array}$

\section{Discussion}

Actual driving performance was affected by alcohol, MDMA and the combination of both substances. MDMA caused a reduction in SDLP (weaving) and SDSP in the road tracking test. Under the influence of both MDMA doses (75 and $100 \mathrm{mg}$ ) SDLP decreased by $2 \mathrm{~cm}$. This is in line with results of a previous study, in which a similar reduction in SDLP after single doses of MDMA was also shown (Ramaekers et al., 2006). Alcohol in contrast, detrimentally affected driving performance by producing an increase in weaving by $2.5 \mathrm{~cm}$ compared to placebo. Alcohol-related increments in SDLP dropped to $1.5 \mathrm{~cm}$ in combination with MDMA $75 \mathrm{mg}$ and even reduced to zero in combination with MDMA $100 \mathrm{mg}$. These data seem to indicate that the stimulatory effect of MDMA on road tracking performance was present both in the absence and in the presence of alcohol, and perhaps even stronger so in the presence of alcohol as suggested by the significant MDMA-Alcohol interaction. Car-following performance however remained unaffected by MDMA while alcohol alone increased brake reaction time. Brake reaction time increased by $20 \mathrm{msec}$ when alcohol was given alone or in combination with $75 \mathrm{mg}$ of MDMA, compared with placebo. When combined with $100 \mathrm{mg}$ MDMA, brake reaction time even increased by $60 \mathrm{msec}$ compared with placebo. These data thus seem to suggest that MDMA may worsen the impairing effects of alcohol on reaction time performance. However, the MDMA by Alcohol interaction term failed to reach significance at the statistical level. In any case, it is clear that MDMA did not reduce the impairing effects of alcohol in the Car-following task. This is in line with previous results showing that the stimulant effects of MDMA were not sufficient to overcome alcohol-induced impairment of impulse control or risk taking behavior (Ramaekers \& Kuypers, 2006).

Psychomotor performance (critical tracking, OMEDA) was not affected by MDMA. It is noteworthy that both critical tracking and OMEDA task performance were shown to change after acute MDMA administration in a previous study by our group (Lamers et al., 2003). That study showed that a single 
dose of MDMA (75 mg) improved tracking performance and decreased the subjects' ability to estimate time to contact in the OMEDA task. The investigators employed a more demanding and complex version of the OMEDA and they measured tracking performance repeatedly between 2 and 5 hours post-dosing, i.e. closer to $\mathrm{T}_{\max }$, which may have increased the sensitivity of these tests for MDMA effects. This explanation however remains highly speculative since MDMA/MDA plasma concentrations in the present study were comparable at the onset and completion of each test session, which suggests that drug levels were stable throughout testing. Alcohol on the other hand significantly impaired critical tracking performance as expected. Combined administration of MDMA and alcohol produced impairments in the Critical tracking task that were comparable to alcohol alone. Alcohol-induced impairment was thus not mitigated by coadministration of MDMA.

Pharmacokinetics of MDMA were expected to be non-linear based on a study of de la Torre and colleagues (2004) who showed that blood plasma concentrations rose non-proportionaly when giving MDMA in doses ranging from $50 \mathrm{mg}$ to $150 \mathrm{mg}$ to a restricted number of subjects. Based on this information, doses in the current study were selected in proximity. Resulting blood plasma concentrations did not reveal an exponential relation. Nevertheless, the values did not differ much from those found by de la Torre and colleagues (2004). They showed actually a more or less linear relation between the $75 \mathrm{mg}$ and $100 \mathrm{mg}$ dose but non-linear relations when taking into account the extreme doses of $50 \mathrm{mg}$ and $150 \mathrm{mg}$. It was also expected that plasma concentrations of MDMA would increase when combined with alcohol (de la Torre et al., 2000). Hernandez-Lopez and colleagues (2002) showed an increase of $13 \%$ when combining a $100 \mathrm{mg}$ dose of MDMA with $0.8 \mathrm{~g} / \mathrm{kg}$ ethanol. Our data showed increases of $7 \%$ and $8.7 \%$ when $75 \mathrm{mg}$, respectively $100 \mathrm{mg}$ MDMA, was combined with alcohol. There was no significant difference between the mean MDMA concentrations in the alcohol condition versus the MDMA only condition for both dose regimes. Mean MDMA concentrations at 1.5 and 5.5 hours after administration of a $75 \mathrm{mg}$ and $100 \mathrm{mg}$ dose, and inter-subject variabilities, were in agreement with data published in other studies (de la Torre et al., 2004; Mas et al., 1999).

When under the influence of alcohol, subjects experienced they had to invest more effort when performing on the driving tasks. Their subjective experience supported objective measures of alcohol impairments of actual driving. Their impression however that they drove better after intake of MDMA, and had to invest less effort was only partly reflected in objective measures: i.e. a reduction 
in SDLP. The results from ARCI were in line with those of a study by Hernandez and colleagues (2002). MDMA alone produced euphoria and stimulant-like effects, and when given in combination with alcohol, MDMA counteracted the effects of the alcohol-induced sedation and lack of energy. In addition, and similar to the results of Hernandez and colleagues (2002), MDMA counteracted the subjective impairment of alcohol but not the objective psychomotor impairment of alcohol.

Collectively these data indicate that MDMA is a stimulant drug that may facilitate a certain aspect of driving i.e. road tracking. However performance compensation after combined MDMA-alcohol administration was limited to a single driving parameter and was never sufficient to fully overcome alcohol impairment in all driver tasks.

\section{Acknowledgments}

We would like to thank Gert De Boeck and Marleen Laloup from NICC, Brussels, for analyzing MDMA blood plasma samples. We also would like to thank. Kirsten Scbuer, Lisa Willems and Anita van Oers for their relative contribution to the study. This work was conducted as part of the IMMORTAL research consortium funded by EU grant GMA12000-27043. 


\section{References}

Barrett, S. P., Gross, S. R., Garand, I., \& Pihl, R. O. (2005). Patterns of Simultaneous Polysubstance Use in Canadian Rave Attendees.

Brookhuis, K. A., de Waard, D., \& Mulder, B. (1994). Measuring driving performance by car-following in traffic. Ergonomics, 37(3), 427-434.

Brookhuis, K. A., De Waard, D., \& Samyn, N. (2004). Effects of MDMA (ecstasy), and multiple drugs use on (simulated) driving performance and traffic safety. Psychopharmacology, 173(3-4), 440-445.

Carmen del Rio, M., Gomez, J., Sancho, M., \& Alvarez, F. J. (2002). Alcohol, illicit drugs and medicinal drugs in fatally injured drivers in Spain between 1991 and 2000. Forensic science international, 127(1-2), 63-70.

de la Torre, R., Farre, M., Ortuno, J., Mas, M., Brenneisen, R., Roset, P. N., et al. (2000). Non-linear pharmacokinetics of MDMA ('ecstasy') in humans. British journal of clinical pharmacology, 49(2), 104-109.

de la Torre, R., Farre, M., Roset, P. N., Pizarro, N., Abanades, S., Segura, M., et al. (2004). Human pharmacology of MDMA: pharmacokinetics, metabolism, and disposition. Therapeutic drug monitoring, 26(2), 137-144.

de Wit, H., Crean, J., \& Richards, J. B. (2000). Effects of d-amphetamine and ethanol on a measure of behavioral inhibition in humans. Behavioral neuroscience, 114(4), 830-837.

Haertzen, C. A. (1965). Addiction Research Center Inventory (ARCI): development of a general drug estimation scale. Journal of nervous and mental disease, 141(3), 300-307.

Henry, J. A. (1992). Ecstasy and the dance of death. BMJ Clinical research ed, 305(6844), 5-6.

Henry, J. A., Jeffreys, K. J., \& Dawling, S. (1992). Toxicity and deaths from 3,4methylenedioxymethamphetamine ("ecstasy"). Lancet, 340(8816), 384-387.

Hernandez Lopez, C., Farre, M., Roset, P. N., Menoyo, E., Pizarro, N., Ortuno, J., et al. (2002). 3,4-Methylenedioxymethamphetamine (ecstasy) and alcohol interactions in humans: psychomotor performance, subjective effects, and pharmacokinetics. $J$ Pharmacol Exp Ther.

Jex, H. R., McDonnell, J. D., \& Phatak, A. V. (1966). A "critical" tracking task for manmachine research related to the operator's effective delay time. I. Theory and experiments with a first-order divergent controlled element. NASA CR-616. NASA Contract Rep NASA CR, 1-105.

Lamers, C. T., Ramaekers, J. G., Muntjewerff, N. D., Sikkema, K. L., Samyn, N., Read, N. L., et al. (2003). Dissociable effects of a single dose of ecstasy (MDMA) on psychomotor skills and attentional performance. Journal of psychopharmacology Oxford, England, 17(4), 379-387. 
Logan, B. K., \& Couper, F. J. (2001). 3,4-Methylenedioxymethamphetamine (MDMA, ecstasy) and driving impairment. Journal of forensic sciences, 46(6), 1426-1433.

Mas, M., Farre, M., de la Torre, R., Roset, P. N., Ortuno, J., Segura, J., et al. (1999). Cardiovascular and neuroendocrine effects and pharmacokinetics of 3, 4methylenedioxymethamphetamine in humans. Journal of pharmacology and experimental therapeutics, 290(1), 136-145.

O'Hanlon, J. F. (1984). Driving performance under the influence of drugs: rationale for, and application of, a new test. British journal of clinical pharmacology, 18 Suppl 1, 121s$129 \mathrm{~s}$.

Parrott, A. C. (2004). Is ecstasy MDMA? A review of the proportion of ecstasy tablets containing MDMA, their dosage levels, and the changing perceptions of purity. Psychopharmacology, 173(3-4), 234-241.

Ramaekers, J. G., \& Kuypers, K. P. C. (2006). Acute Effects of 3,4Methylenedioxymethamphetamine (MDMA) on Behavioral Measures of Impulsivity: Alone and in Combination with Alcohol. Neuropsychopharmacology official publication of the American College of Neuropsychopharmacology, 31(5), 1048-1055.

Ramaekers, J. G., Kuypers, K. P. C., \& Samyn, N. (2006). Stimulant effects of 3,4methylenedioxymethamphetamine (MDMA) $75 \mathrm{mg}$ and methylphenidate $20 \mathrm{mg}$ on actual driving during intoxication and withdrawal. Addiction, DOI:10.1111/j.1360-0443.2006.01566.x,1-8.

Ramaekers, J. G., Robbe, H. W. J., \& O'Hanlon, J. F. (2000). Marijuana, alcohol and actual driving performance. Human Psychopharmacology Clinical and Experimental, 15(7), 551 558.

Read, N., Ward, N., \& Parkes, A. (2000). The role of dynamic tests in assessing the fitness to drive of healthy and cognitively impaired elderly. Journal of Traffic Medicine, 28, 34-35S.

Riley, S. C., James, C., Gregory, D., Dingle, H., \& Cadger, M. (2001). Patterns of recreational drug use at dance events in Edinburgh, Scotland. Addiction Abingdon, England, 96(7), 1035-1047.

Schifano, F. (1995). Dangerous driving and MDMA ("Ecstasy") abuse. Journal of Serotonin Research, 1, 53-57.

Topp, L., Hando, J., Dillon, P., Roche, A., \& Solowij, N. (1999). Ecstasy use in Australia: patterns of use and associated harm. Drug and alcohol dependence, 55(1-2), 105-115. Zijlstra, F. (1993). Efficiency in work behavior. A design approach for modern tools., University of Technology, Delft, The Netherlands. 
148 | CHAPTER 8 


\section{CHAPTER 9}

\section{GENERAL DISCUSSION}

\section{Selectivity of the memory effect}

In chapters 2 and 3 it was shown that impairment of verbal, and spatial memory was present $1.5 \mathrm{~h}$ after administration of a single dose of MDMA. This impairment was also present after a repeated dose of MDMA in combination with sleep deprivation (chapter 4). The other cognitive tasks assessed in those studies (chapters 2, 3 and 4) were not negatively affected by a single or repeated dose of MDMA. This is a strong indication that the impairing effects of MDMA on memory performance were not due to a general effect on attention or motivation, but were rather selective.

The impairment after an acute dose resembles the deficits seen in abstinent and/or current users of MDMA. This concordance gives an indication that the observed deficits in abstinent and/or current users were actually caused by MDMA, and may share a common psychopharmacological mechanism. Although the mechanism by which the chronic memory impairment is caused is unknown, the mechanism underlying acute impairment is partly known. As shown in chapter 2 , there was no memory impairment after treatment with a dopamine transporter blocker i.e. methylphenidate. Consequently, the memory impairment caused by MDMA is less likely to be attributable to its actions on the dopaminergic system. An obvious culprit is serotonin (5-HT), as it is known that this neurotransmitter is involved in memory processes. MDMA, as a powerful, indirect 5-HT agonist disrupts the normal functioning of the serotonergic system. The disruption of 5HT affects also other systems involved in normal memory functioning i.e. glucocorticoid hormones (i.e. cortisol) (van Praag, 2004). Studies have shown that at low doses, and post-training, glucocorticoid hormones can enhance memory consolidation. At high doses however, glucocorticoglucoids can impair memory performance, although not permanently (Andreano \& Cahill, 2006; Brunner et al., 2006). Increased levels of 5-HT have also been shown to increase corticosteroid release (van Praag, 2004). Lock and colleagues (2006) showed that levels of cortisol were significantly increased in ecstasy users after self-administration of MDMA, in a club (Lock, Kissling, Thome, \& Parrott, 2006). The increase in cortisol levels can cause the $5-\mathrm{HT}_{1 \mathrm{a}}$ receptor system to be more responsive (van Praag, 2004). These 
$5-\mathrm{HT}_{1 \mathrm{a}}$ receptors are implicated in memory performance, with more stimulation being related to poorer memory performance (Meneses \& Hong, 1997; Yasuno et al., 2003). This increased availability of 5-HT in addition to the augmented responsiveness of the $5-\mathrm{HT}_{1 \mathrm{a}}$ receptors would explain the memory deficits caused by an acute dose of MDMA. It would be interesting to study the involvement of the $5-\mathrm{HT}_{1 \mathrm{a}}$ receptor in relation with the MDMA-induced memory impairment. In future research, a selective $5-\mathrm{HT}_{1 \mathrm{a}}$ receptor blocker could be used in combination with MDMA to see whether memory deficits would still be present $1.5 \mathrm{~h}$ after administration of MDMA. Simultaneous measures of cortisol levels could provide additional information.

Another potential mechanism underlying memory impairment in MDMA users is hippocampal dysfunction due to chronic, or repeated acute stress-level cortisol elevations (Riedel, Sobczak, Nicolson, \& Honig, 2002). It has been suggested that intense use of MDMA within a session, or repeated use, leads to repetitive high levels of cortisol. Additionally, factors related to the party lifestyle i.e. heat (as a result of dancing in hot places), can influence the glucocorticoid system and lead to elevated cortisol levels (McMorris et al., 2006). This could cause structural changes in the memory system with functional impairment as a result.

\section{Is MDMA a neurotoxin?}

A topic of societal importance, and major concern, is whether MDMA is truly a neurotoxin. Many animal studies have reported on structural changes in the brains of non-(human) primates after exposure to MDMA (Green, Mechan, Elliott, O'Shea, \& Colado, 2003; Parrott, 2001). Non-human primates appear to be more sensitive to the neurotoxic effects of MDMA compared with rodents as shown by the presence of more persistent structural changes. The functional changes in all animal species tested however appear to be subtle (Gouzoulis Mayfrank \& Daumann, 2006). It can be questioned how these findings from animal models translate to humans because animal models differ in a number of aspects compared with humans e.g. MDMA dosage, pattern of dosing, and route of administration (Gouzoulis Mayfrank \& Daumann, 2006). Irvine and colleagues (2006) investigated whether the plasma concentrations of MDMA in humans at dance parties were comparable with those in animals causing structural damage. It appeared that in a number of subjects, blood plasma concentrations were equivalent to those which caused structural damage in animals (Irvine et al., 2006). However, the concentrations in animals were two to three times higher compared 
with those of humans receiving a 100-150 mg dose under controlled circumstances (Mechan et al., 2006). Additionally, it remains highly debatable whether this dosescaling from animal models to humans is meaningful because pharmacokinetic profiles between species differ (De La Torre \& Farre, 2004). An example of this is that the half-life of MDMA differs between non-human primates, and humans, and it was suggested that sustained concentrations of MDMA in blood could contribute to the origin of structural changes. Furthermore, it appeared that a single dose of MDMA resulted in higher plasma concentrations in non-human primates compared with humans. This could be due to the scaling that is used between species (Mechan et al., 2006). Obviously it remains difficult to make generalizations from animal models to humans (Easton \& Marsden, 2006)

Reports of structural and functional changes also exist in ecstasy users (Parrott, 2001). An example of structural changes is the lower densities of the serotonin transporter that are being found in the brains of heavy ecstasy users compared with non-users. These lowered densities are dose-related and thought to be of temporary nature (Reneman, de Win, van den Brink, Booij, \& den Heeten, 2006). Also, the development of chronic tolerance to MDMA could be an indication for structural changes (modified receptor sensitivity) or perhaps even structural damage (Parrott, 2005). That is, structural brain changes following repeated use may precipitate the need for higher doses in order to achieve desired drug effects. Ecstasy users describe their first time experience as the best, and experience with continued use the need to augment their normal dose in order to experience beneficial mood effects (Parrott, 2005). Of course, these structural changes are not unique to ecstasy use alone as it is shown for example that certain receptors in depressed patients adjust in sensitivity after treatment with SSRI's (Raap \& Van de Kar, 1999). These (temporarily) structural changes are therefore no evidence for ecstasy's potential neurotoxicity. However the existence of functional disturbances i.e. cognitive disturbances (memory, decision making, impulsivity), and psychiatric problems may be a strong indication for the existence of neuronal damage (Cole \& Sumnall, 2003; Morgan, McFie, Fleetwood, \& Robinson, 2002; Parrott, 2001). Recent studies have shown that impairment of verbal memory was even present in ecstasy users that had been abstinent for over 2 years compared with their performance on previous measures and/or compared with a control group (Thomasius et al., 2006; Ward, Hall, \& Haslam, 2006).

These human studies are however loaded with methodological problems which hamper the inference of MDMA being a neurotoxin or not (Curran, 2000; Turner \& Parrott, 2000). Confounding factors like poly-drug use, impurity of 
tablets, or possible predisposition to cognitive and/or psychiatric deficits can act as confounders (Easton \& Marsden, 2006; Lieb, Schuetz, Pfister, von Sydow, \& Wittchen, 2002). Reneman and colleagues tried to circumvent at least one of those problems with their latest prospective longitudinal cohort study. They are currently seeing young subjects who are not using ecstasy but have a high probability of starting to use the drug in the future. De Win and colleagues (2006) presented already some data from this prospective cohort study. They were interested to investigate the relation between first time ecstasy use and some psychological measures i.e. depression, impulsivity. It appeared that low level ecstasy use did not cause depression or impulsivity (de Win et al., 2006). They can however not control for problems like impurity of the tablets or poly-drug use. In chapters 2, and 3 of this dissertation however, these problems were circumvented by using an acute, placebo-controlled design. It was shown that a single dose of MDMA caused impairment of verbal and spatial memory, 1.5 hours post-ingestion.

The fact that the impairment was gone 25.5 hours post-ingestion is a good indication that a single dose of MDMA causes no permanent damage in light and moderate ecstasy users. It can therefore be assumed that a single dose of ecstasy causes no persistent functional damage. Longitudinal prospective cognitive neuroimaging studies are needed to shed more light on the issue whether repeated, chronic use causes irreversible functional and structural damage.

\section{MDMA use, impulsivity, and driving}

In chapter 6,7 , and/ or 8 the effects of MDMA alone, and in combination with a moderate dose of alcohol, were assessed on measures of psychomotor performance, impulse control, decision making, and actual driving performance. Driving performance was tested between 3 and 5 hours post-dosing by means of two driving tasks, each manipulating a different level of driving performance. The driving task can be sub-divided into three hierarchically organized levels depending on demands put on the driver. On the highest, strategically level, route planning and speed choice are defined. On the tactical level, maneuver control is exercised by the driver -negotiating with traffic signs, other traffic participants, keeping following distance, changing traffic lanes, and so forth. At the lowest, operational level, automatic action patterns, e.g. to maintain lane-position, are carried out by the driver (Al-Shihabi, 2001; Brookhuis, de Vries, \& de Waard, 1991). Standard deviation of lateral position (SDLP), the primary outcome of the Road tracking task, a measure of weaving, and psychomotor performance correspond with 
performance on the operational level. The outcome variables of the Car-following task are measures of driving performance at the tactical level. Measures of impulse control, and decision making performance, conducted between 1.5 and 2.5 hours post-dosing, were included to measure aspects related to driving at the tactical (e.g. Stop-signal task), and the strategically (e.g. Iowa gambling task) level, and give additional information to outcomes of the actual driving tests.

In chapter 8 it was shown that a single dose of MDMA affected automated driving performance, as indicated by a reduction of weaving compared with placebo (measured by the Road tracking task). In addition, when combined with alcohol, MDMA seemed to partially counteract the impairing effects of alcohol on weaving. Performance on the tactical level (as measured by the Carfollowing task) however was still impaired when alcohol was combined with MDMA. Evidently the stimulating effects of MDMA were only present when the driving task was relatively easy, and monotonous. When more demands were put on the driver, i.e. interaction with a leading car, in the car-following task, MDMA could not overcome the impairing influence of alcohol on performance on the tactical level. The fact that MDMA could not compensate for the impairing effects of a low dose of alcohol was also demonstrated by a measure of impulsivity. The alcohol-induced impairment of inhibition on a visual stop signal was not counteracted by MDMA. In real traffic this would mean that a person under the influence of MDMA and alcohol is not able to brake when a traffic light turns from green/orange to orange/red. This could create unsafe situations for the driver, and the other persons on the road.

In sum, the stimulating effects of two particular doses of MDMA were found only on one level of the driving task (operational) under optimal driving conditions i.e. daytime, lower than average speed compared to other traffic, low level of interaction with traffic. In real life on the other hand, conditions are not that optimal as people tend to combine several substances, perhaps take additional doses of ecstasy and drive at nighttime at higher speeds than the set limit during the driving test. These are all factors that could affect different levels of the driving task in a negative way.

In chapters 6,7 , and 8 it was shown that several measures of cognitive and psychomotor performance after a dose of MDMA in combination with an arousal/activation-lowering manipulation, i.e. a low dose of alcohol or sleep deprivation, were impaired. Measures of subjective impairment however demonstrated that subjects did not notice this impairment. This discrepancy between objective, and subjective measures is an issue that deserves extra 
attention. In real life, subjects tend to rely on subjective feelings to decide whether they find themselves able to drive a car. In chapters 7 and 8 , subjective measures have shown that subjects are not able to self-judge the extent of their objective impairment. This can be very dangerous, certainly when subjects have mixed several drugs, are sleep deprived, and want to drive home after a party which is the situation every weekend.

\section{Suggestions for further/future research}

Extra control group. It could be argued that the addition of a healthy, drug-naïve, matched, norm-reference control group to the existing 'experimental withinsubject'-format might create a surplus value, although this is debatable. An advantage of the addition of this group (which does not receive any treatment) is that it can be validated that ecstasy users in the placebo condition were functioning at a normal cognitive level. A disadvantage however can be that with the inclusion of a control group, methodological problems (confounders) are introduced. To prevent confounding, the control group has to share as many characteristics with the subject in the experimental condition, i.e. age, education. The experimental group may have different, unknown characteristics, which distinguishes it from the control group. When it is very difficult to find a perfect match it is questionable whether it is really a surplus or rather an extra factor creating confusion.

With respect to the samples used in the studies presented in this thesis, it can be said that all subjects were healthy as ascertained by a medical examination, they were merely light users, and to the larger part university students. The performance of these subjects on tasks in the placebo condition appeared to be normal as compared with the cognitive, and psychomotor performance of healthy, same aged subject-groups, on comparable tasks (Theunissen, van Kroonenburgh, van Deursen, Blom Coenjaerts, \& Ramaekers, 2006; Theunissen, Vermeeren, \& Ramaekers, 2006; Theunissen, Vermeeren, van Oers, van Maris, \& Ramaekers, 2004; Wingen, Bothmer, Langer, \& Ramaekers, 2005; Wingen, Kuypers, \& Ramaekers, 2006a, 2006b; Wingen \& Ramaekers, 2006).

Poly-drug use. As mentioned earlier, ecstasy users are mainly poly-drug users who tend to combine ecstasy with substances such as cannabis, alcohol, amphetamines, and cocaine but also hallucinogens, GHB, and opiates (Tossmann, Boldt, \& Tensil, 2001; Uys \& Niesink, 2005; Winstock, Griffiths, \& Stewart, 2001). The combination of alcohol with MDMA has previously been investigated by a number 
of studies. In chapters 6 and 8 , the effects of the combination of a moderate dose of alcohol with a single dose of MDMA on psychomotor, and impulsivity measures, and actual driving performance, were presented. Overall, MDMA did not modulate the impairing effects of alcohol on these measures except for one, i.e. a parameter of automated driving performance (longitudinal and lateral control of a vehicle). This was not the result of a pharmacokinetic interaction as the blood alcohol concentrations with, and without MDMA, and the plasma concentrations of MDMA with, and without alcohol, did not differ significantly.

In the future, studies combining MDMA with THC or amphetamines could provide very useful information because these substances have mainly different pharmacodynamic properties, and therefore the effects on psychomotor and cognitive measures could be widespread. This could have serious implications in real life, when people try to conduct a vehicle under the influence of this party cocktail of drugs.

When studying the effects of these combinations it would also be interesting to add some factors related to the 'party lifestyle' of ecstasy users i.e. sleep deprivation, and prolonged physical exertion, to increase the ecological validity of the experimental studies. The studies described in this dissertation mainly chapters $3,5,7$, and 8 , combining ecstasy with alcohol or sleep deprivation, are the stepping-stones to future real life studies combining several drugs, and reallife factors (i.e. sleep deprivation and physical exertion).

Mechanistic studies. When taking the cognitive and psychomotor effects of MDMA into consideration, it is not certain which neurotransmitter was responsible for the observed acute effects. It is known that MDMA exerts it main effects through the serotonergic system. Additionally, it also affects dopaminergic, and to a lesser extent cholinergic, noradrenergic, and histaminergic systems. It is most likely that cognitive effects are caused by effects on the serotonergic system, and possibly that some psychomotor effects are primarily caused by manipulations of the dopaminergic system. In the studies presented in chapters 2 and 3, a dopaminergic agent (methylphenidate) was used as an active control to determine whether MDMA-induced effects were mediated by dopamine and/ or serotonin. Overall, methylphenidate demonstrated no effects on the behavioural measures, and therefore it can be assumed that the memory impairing effects, caused by MDMA, were due to a manipulation of the 5-HT system.

There are several ways to study the contribution of respective neurotransmitters to the resulting behaviors following MDMA ingestion in 
humans. One way could be to combine depletion techniques with the administration of ecstasy, i.e. tryptophan, and tyrosine depletion (Riedel, Klaassen, Deutz, van Someren, \& van Praag, 1999); (Harmer, Mc'Tavish, Clark, Goodwin, \& Cowen, 2001). Tryptophan and tyrosine are both precursors for the synthesis of serotonin, and dopamine, and eventually (nor)adrenaline respectively. Behavioral effects resulting after treatment with tryptophan depletion and MDMA administration could not be contributable to 5-HT. In addition, when MDMA administration would be preceded by tyrosine depletion, behavioral effects would essentially result from the 5-HT release, caused by MDMA. This technique has the advantage that it scores low on the scale of invasiveness.

Another, though more invasive technique to unravel the contributions of respective neurotransmitters would be by means of neuro-imaging, i.e. PET or SPECT in combination with MDMA administration. Ideal would be to use different kind of tracers to visualize 5-HT and DA-transporters in different sessions, within one subject. This is however not approved because of health related matters (radiation). The obliged use of a between subjects design limits the strength of the findings; however it would nevertheless provide valuable information.

\section{Conclusion}

The studies presented in this dissertation were the first placebo-controlled withinsubject studies showing a causal relation between MDMA, and memory impairment. Furthermore it was shown that single dose of MDMA produced improved impulse control. When combined with a moderate dose of alcohol or sleep deprivation however, MDMA was not able to counteract the impairing effects of these additional manipulations on psychomotor, and impulsivity measures. Moreover, nocturnal doses of MDMA produced impairment of psychomotor performance that was additive to sleep loss-induced impairment.

The memory impairment caused by a single, and a repeated dose of MDMA was selective i.e. other tasks were not affected. Moreover, it was temporary as it was no longer visible in the withdrawal period i.e. 25.5 hours postdrug. Abstinent and/or current users also display memory deficits and the concordance with results from the present dissertation let us suggest that perhaps a similar mechanism underlies this impairment. It was suggested that $5-\mathrm{HT}_{1 \mathrm{a}}$ receptors in combination with elevated levels of cortisol, and chronic stress may 
contribute to this memory impairment. Future mechanistic studies can perhaps provide additional evidence for this notion.

With respect to MDMA, and psychomotor performance, and impulse control, it would be interesting to include additional experimental factors (combinations of drugs, physical exertion) in future research to increase the ecological validity of controlled laboratory studies, and consequently to gain more insight in the real-life situation.

The studies presented in this dissertation are unique, provide new information, and they are the stepping-stones to further profound research in the area of MDMA use, cognition, and psychomotor behavior. 


\section{References}

Al-Shihabi, T., Mourant, R.R. (2001). A framework for modeling human-like driving behaviors for autonomous vehicles in driving simulators. Proceedings 5 th international conference on autonomous agents, 286-291.

Andreano, J. M., \& Cahill, L. (2006). Glucocorticoid release and memory consolidation in men and women. Psychological science a journal of the American Psychological Society APS, 17(6), 466-470.

Brookhuis, K. A., de Vries, G., \& de Waard, D. (1991). The effects of mobile telephoning on driving performance. Accident analysis and prevention, 23(4), 309-316.

Brunner, R., Schaefer, D., Hess, K., Parzer, P., Resch, F., \& Schwab, S. (2006). Effect of high-dose cortisol on memory functions. Annals of the New York. Academy of Sciences, 1071, 434-437.

Cole, J. C., \& Sumnall, H. R. (2003). Altered states: the clinical effects of Ecstasy. Pharmacology and Therapeutics, 98, 35-58.

Curran, H. V. (2000). Is MDMA ('Ecstasy') neurotoxic in humans? An overview of evidence and of methodological problems in research. Neuropsychobiology, 42(1), 3441.

De La Torre, R., \& Farre, M. (2004). Neurotoxicity of MDMA (ecstasy): the limitations of scaling from animals to humans. Trends in pharmacological sciences, 25(10), 505-508.

de Win, M. M., Schilt, T., Reneman, L., Vervaeke, H., Jager, G., Dijkink, S., et al. (2006). Ecstasy use and self-reported depression, impulsivity, and sensation seeking: a prospective cohort study. Journal of psychopharmacology Oxford, England, 20(2), 226235.

Easton, N., \& Marsden, C. A. (2006). Ecstasy: are animal data consistent between species and can they translate to humans? Journal of psychopharmacology Oxford, England, 20(2), 194-210.

Gouzoulis Mayfrank, E., \& Daumann, J. (2006). Neurotoxicity of methylenedioxyamphetamines (MDMA; ecstasy) in humans: how strong is the evidence for persistent brain damage? Addiction Abingdon, England, 101(3), 348361.

Green, A. R., Mechan, A. O., Elliott, J. M., O'Shea, E., \& Colado, M. I. (2003). The pharmacology and clinical pharmacology of 3,4methylenedioxymethamphetamine (MDMA, "ecstasy"). Pharmacological reviews, 55(3), 463-508. 
Harmer, C. J., McTavish, S. F., Clark, L., Goodwin, G. M., \& Cowen, P. J. (2001). Tyrosine depletion attenuates dopamine function in healthy volunteers. Psychopharmacology, 154(1), 105-111.

Irvine, R. J., Keane, M., Felgate, P., McCann, U. D., Callaghan, P. D., \& White, J. M. (2006). Plasma drug concentrations and physiological measures in 'dance party' participants. Neuropsychopharmacology official publication of the American College of Neuropsychopharmacology, 31(2), 424-430.

Lieb, R., Schuetz, C. G., Pfister, H., von Sydow, K., \& Wittchen, H. (2002). Mental disorders in ecstasy users: a prospective-longitudinal investigation. Drug and alcohol dependence, 68(2), 195-207.

Lock, j., Kissling, C., Thome, J., \& Parrott, A. C. (2006). Cortisol, testosterone, and mood changes in ecstasy/MDMA users at a saturday night dance club: a brief prospective study. Paper presented at the British Association for Psychopharmacology, Oxford.

McMorris, T., Swain, J., Smith, M., Corbett, J., Delves, S., Sale, C., et al. (2006). Heat stress, plasma concentrations of adrenaline, noradrenaline, 5-hydroxytryptamine and cortisol, mood state and cognitive performance. International journal of psychophysiology official journal of the International Organization of Psychophysiology, 61(2), 204-215.

Mechan, A., Yuan, J., Hatzidimitriou, G., Irvine, R. J., McCann, U. D., \& Ricaurte, G. A. (2006). Pharmacokinetic profile of single and repeated oral doses of MDMA in squirrel monkeys: relationship to lasting effects on brain serotonin neurons. Neuropsychopharmacology official publication of the American College of Neuropsychopharmacology, 31(2), 339-350.

Meneses, A., \& Hong, E. (1997). Role of 5-HT-sub(1A ) receptors in acquisition, consolidation and retrieval of learning. CNS Drug Reviews, 3(1), 68-82.

Morgan, M. J., McFie, L., Fleetwood, H., \& Robinson, J. A. (2002). Ecstasy (MDMA): are the psychological problems associated with its use reversed by prolonged abstinence? Psychopharmacology, 159(3), 294-303.

Parrott, A. C. (2001). Human psychopharmacology of Ecstasy (MDMA): A review of 15 years of empirical research. Human Psychopharmacology Clinical and Experimental, 16(8), 557-577.

Parrott, A. C. (2005). Chronic tolerance to recreational MDMA (3,4methylenedioxymethamphetamine) or Ecstasy. Journal of psychopharmacology Oxford, England, 19(1), 71-83.

Raap, D. K., \& Van de Kar, L. D. (1999). Selective serotonin reuptake inhibitors and neuroendocrine function. Life sciences, 65(12), 1217-1235. 
Reneman, L., de Win, M. M., van den Brink, W., Booij, J., \& den Heeten, G. J. (2006). Neuroimaging findings with MDMA/ecstasy: technical aspects, conceptual issues and future prospects. Journal of psychopharmacology Oxford, England, 20(2), 164-175.

Riedel, W. J., Klaassen, T., Deutz, N. E., van Someren, A., \& van Praag, H. M. (1999). Tryptophan depletion in normal volunteers produces selective impairment in memory consolidation. Psychopharmacology, 141(4), 362-369.

Riedel, W. J., Sobczak, S., Nicolson, N., \& Honig, A. (2002). Stress, cortisol and memory as markers of serotonergic vulnerability. Acta neuropsychiatrica, 14, 186-191.

Theunissen, E. L., van Kroonenburgh, M. J., van Deursen, J. A., Blom Coenjaerts, C., \& Ramaekers, J. G. (2006). Stimulating effects of the antihistamine fexofenadine: testing the dopamine transporter hypothesis. Psychopharmacology, 187(1), 95-102.

Theunissen, E. L., Vermeeren, A., \& Ramaekers, J. G. (2006). Repeated-dose effects of mequitazine, cetirizine and dexchlorpheniramine on driving and psychomotor performance. British journal of clinical pharmacology, 61(1), 79-86.

Theunissen, E. L., Vermeeren, A., van Oers, A. C., van Maris, I., \& Ramaekers, J. G. (2004). A dose-ranging study of the effects of mequitazine on actual driving, memory and psychomotor performance as compared to dexchlorpheniramine, cetirizine and placebo. Clinical and experimental allergy journal of the British Society for Allergy and Clinical Immunology, 34(2), 250-258.

Thomasius, R., Zapletalova, P., Petersen, K., Buchert, R., Andresen, B., Wartberg, L., et al. (2006). Mood, cognition and serotonin transporter availability in current and former ecstasy (MDMA) users: The longitudinal perspective.

Tossmann, P., Boldt, S., \& Tensil, M. D. (2001). The use of drugs within the techno party scene in European metropolitan cities. European addiction research, 7(1), 2-23.

Turner, J. J., \& Parrott, A. C. (2000). 'Is MDMA a human neurotoxin?': diverse views from the discussants. Neuropsychobiology, 42(1), 42-48.

Uys, J. D., \& Niesink, R. J. (2005). Pharmacological aspects of the combined use of 3,4methylenedioxymethamphetamine (MDMA, ecstasy) and gamma-hydroxybutyric acid (GHB): a review of the literature. Drug and alcohol review, 24(4), 359-368.

van Praag, H. M. (2004). Can stress cause depression? Progress in neuro psychopharmacology and biological psychiatry, 28(5), 891-907.

Ward, J., Hall, K., \& Haslam, C. (2006). Patterns of memory dysfunction in current and 2year abstinent MDMA users. Journal of clinical and experimental neuropsychology official journal of the International Neuropsychological Society, 28(3), 306-324.

Wingen, M., Bothmer, J., Langer, S., \& Ramaekers, J. G. (2005). Actual driving performance and psychomotor function in healthy subjects after acute and 
subchronic treatment with escitalopram, mirtazapine, and placebo: a crossover trial. Journal of clinical psychiatry, 66(4), 436-443.

Wingen, M., Kuypers, K. P. C., \& Ramaekers, J. G. (2006a). The role of 5-HT1a and 5HT2a receptors in attention and motor control: a mechanistic study in healthy volunteers. Psychopharmacology, submitted.

Wingen, M., Kuypers, K. P. C., \& Ramaekers, J. G. (2006b). Selective verbal and spatial memory impairment after 5-HT1 a and 5-HT2a receptor blockade in healthy volunteers pre-treated with an SSRI. Journal of psychopharmacology Oxford, England, accepted.

Wingen, M., \& Ramaekers, J. G. (2006). Verbal memory performance during subchronic challenge with a selective serotonergic and mixed action depressant. Human psychopharmacology, accepted.

Winstock, A. R., Griffiths, P., \& Stewart, D. (2001). Drugs and the dance music scene: a survey of current drug use patterns among a sample of dance music enthusiasts in the UK. Drug and alcohol dependence, 64(1), 9-17.

Yasuno, F., Suhara, T., Nakayama, T., Ichimiya, T., Okubo, Y., Takano, A., et al. (2003). Inhibitory effect of hippocampal 5-HT-sub(1A ) receptors on human explicit memory. American Journal of Psychiatry, 160(2), 334-340. 
$162 \mid$ CHAPTER 9 


\section{SUMMARY}

Ecstasy (MDMA) is an illegal drug, which is very popular among youngsters, frequently visiting large dance parties. It has been estimated recently that incidence of drug use among young adults (15-34 years) in Europe is currently between 0.6 and $6 \%$. Given the widespread and frequent use of ecstasy, the number of cases with acute medical problems is yet low. Nevertheless, serious concern has been raised by possible long-term effects of ecstasy use on cognitive functioning. Numerous studies investigating cognitive performance, e.g. memory, of ecstasy users have shown that (current/abstinent) ecstasy users have impaired memory performance and display elevated levels of impulsivity in comparison with a reference group, i.e. polydrug users or cannabis users. The problem however is that these differences in performance may be due to confounding demographic variables (e.g. different lifestyle, premorbid undiagnosed psychopathology). Consequently the question remains whether the reported cognitive problems are caused by ecstasy itself or by other factors related to ecstasy use. The major aim of the studies presented in this thesis was to investigate if there is a causal association between ecstasy use and impaired cognitive performance and to elucidate its psychopharmacological mechanism. The basic experimental design to investigate this was a placebo-controlled, within-subject design. In the first four chapters following the introduction, four experiments investigating the relation between MDMA and memory impairment are described. The next three experimental chapters focused on the relation between MDMA and degree of impulsivity and psychomotor functioning.

Chapter 1 gives an overview of the pharmacodynamic and pharmacokinetic effects of ecstasy (MDMA) and mentions limitations of existing research. These limitations are being discussed and an alternative research design that circumvents these problems is presented.

Chapter 2 presents the results of a study that was designed to establish the direct relation between MDMA and memory impairment under placebo-controlled conditions. Eighteen recreational ecstasy users participated in a double-blind, placebo-controlled, three-way crossover design. They were treated with placebo, 75 $\mathrm{mg}$ of MDMA and $20 \mathrm{mg}$ of methylphenidate. Memory tests were conducted between 1.5-2 hours (intoxication phase) and between 25.5-26 hours (withdrawal phase) post dosing. Results showed that a single dose of MDMA caused impairment of immediate and delayed recall on a verbal learning task during the 
intoxication phase. The next day, during the withdrawal phase, there was no residual memory impairment. Subjects reported more fatigue and less vigor, but no symptoms of depression during the MDMA withdrawal phase. Methylphenidate did not affect memory or mood at any time of testing. It was concluded that a single dose of MDMA produces transient memory impairment.

Chapter 3. The study described in this chapter was designed to assess whether a single dose of MDMA affects spatial memory performance during intoxication and withdrawal phase and second, whether spatial memory performance following MDMA is task-dependent. Eighteen recreational ecstasy users participated in a double-blind, placebo-controlled, three-way cross-over design. They were treated with placebo, MDMA $75 \mathrm{mg}$ and methylphenidate $20 \mathrm{mg}$. Memory tests were conducted between 1.5-2 hours (intoxication phase) and between 25.5-26 hours (withdrawal phase) post-dosing. Two spatial memory tasks of varying complexity were used that required either storage of stimulus location alone (Spatial memory task), or memory for location as well as processing of content or contextual information (Change blindness task). Results showed that after a single dose of MDMA, subjects made larger localization errors and responded faster compared with placebo in the simple spatial memory task during intoxication phase. Inaccuracy was not due to increased response speed, as determined by regression analysis. Performance in the Change blindness task was not affected by MDMA. Methylphenidate did not affect performance on any of the tasks. It was concluded that a single dose of MDMA impairs spatial memory for location but leaves processing of contextual information intact.

The study presented in chapter 4 was designed to investigate whether evening doses of MDMA added to, or interacted with the memory impairment due to sleep deprivation. Fourteen healthy subjects participated in a double-blind, placebocontrolled, two-way cross-over study. Treatments consisted of MDMA $75 \mathrm{mg}$ and $50 \mathrm{mg}$, administered at 8 PM and 12 PM respectively, or double placebo. Memory tests and subjective measures of mood were conducted at baseline and three times post-dosing i.e. at 6.30 PM, 9.30 PM, 1.30 AM and $7 \mathrm{AM}$, respectively -1.5, 1.5, 5.5 and 11 hours relative to drug intake ( $1^{\text {st }}$ dose). Memory performance detoriated progessively over time as a function of sleep loss, independent of treatment. MDMA added to this impairment as indicated by a significant main effect of MDMA on verbal and spatial memory performance. Mood ratings and response speed revealed an MDMA by Time interaction. After administration of MDMA response speed improved and feelings of vigor, friendliness, elation, anxiety, 
confusion, arousal and positive mood increased in magnitude during the night, while all these parameters returned to placebo-like levels on the final morning session. It is concluded that evening doses of MDMA selectively impair memory performance, and that this impairment is additional to the effect of sleep deprivation on memory performance.

Chapter 5. The study described in this chapter was designed to investigate the neural substrates underlying MDMA-induced memory impairment. Seven healthy male recreational MDMA users participated in the study. The study was conducted according to a double-blind, placebo-controlled within-subject design. Treatments were $75 \mathrm{mg}$ of MDMA and placebo. Prior to drug-intake, subjects had to memorize a list of 15 words (control list). One and a half hours after drug administration, they were placed in a 3-Telsa scanner. In the scanner, they were presented two word lists each in alternation. One list was the control list and the other the experimental list, containing 15 new words. Subjects' task was to read the words quietly and learn them. Thirty-second blocks of the experimental list were alternated with 30-second blocks of the control list and this procedure was repeated five times. After each list-presentation, subjects had to recall as much words as possible. Behavioral data included the number of words correctly recalled on each trial, and their sum over the 5 trials. Functional data consisted of recordings of the BOLD signal during presentation of both lists. Word-learning was mainly related with activation patterns in frontal regions. Under influence of MDMA, the fMRI response increased independent of list-type in the left medial frontal area, which is involved in word-learning. On the behavioural level, memory performance tended to be lower under the influence of MDMA, but failed to reach statistical significance. It was shown that a single dose of MDMA caused an increase in BOLD activity in the experimental as well as the control list condition. This seems to indicate that the left medial frontal area which is involved in word learning underlies memory impairment observed at the behavioral level. The preliminary conclusion is that MDMA affects verbal memory functioning by interfering with the word-learning or encoding phase.

The study presented in chapter 6 was designed to examine the acute effects of MDMA and alcohol, alone and in combination, on behavioural measures of impulsivity and risk-taking behaviour. Eighteen recreational ecstasy users entered a double-blind, placebo-controlled, six-way cross-over study. The treatments consisted of MDMA 0, 75 and $100 \mathrm{mg}$ with and without alcohol. Alcohol dosing was designed to achieve a peak blood alcohol concentration (BAC) of about 0.06 
$\mathrm{g} / \mathrm{dl}$ during laboratory testing. Laboratory tests of impulsivity were conducted between 1.5 and 2 hours post-MDMA and included a Stop-signal task, a Go/no-go task and the Iowa gambling task. MDMA decreased stop reaction time in the Stopsignal task indicating increased impulse control. Alcohol increased the proportion of commission errors in the Stop-signal task and the Go/no-go task. Signal detection analyses of alcohol-induced commission errors indicated that this effect may reflect impairment of perceptual or attentive processing rather than an increase of motor impulsivity per se. Performance in the Iowa gambling task was not affected by MDMA and alcohol, but there was a non-significant tendency towards improvement following alcohol. None of the behavioral measures of impulsivity showed a MDMA by alcohol interaction effect. The lack of interaction indicated that the CNS stimulant effects of MDMA where never sufficient to overcome alcohol-induced impairment of impulse control or risk-taking behavior.

Chapter 7 presents a study that was designed to investigate the effects of nocturnal doses of MDMA during the night and after a night of sleep deprivation, on psychomotor performance and impulsivity. Fourteen healthy subjects participated in a double-blind, placebo-controlled, two-way cross-over study. Treatments consisted of MDMA 75 and $50 \mathrm{mg}$, administered at $8 \mathrm{PM}$ and $12 \mathrm{PM}$ respectively, or double placebo. Laboratory tests (psychomotor and impulsivity tasks) were conducted four times throughout the evening and night i.e. at 6.30 PM, 9.30 PM, 1.30 AM and $7 \mathrm{AM}$, respectively -1.5, 1.5, 5.5 and 11 hours post dosing (1 st dose). A sleepiness scale was presented to the subjects 10 times throughout the evening and night to assess their current level of sleepiness. MDMA impaired tracking performance in a simple tracking task. In addition, divided attention task performance was also impaired as indicated by a decrease in secondary task performance under influence of MDMA, compared with placebo. MDMA did not affect measures of motor and cognitive impulsivity. Vigilance performance (at 5 $\mathrm{AM}$ ) decreased as a function of time on task, but this decrement was less during MDMA treatment as compared to placebo. After administration of MDMA, subjects scored lower on the sleepiness scale during the night when compared with placebo. This difference between MDMA and placebo disappeared in the morning, when scores were equally high in both conditions. It is concluded that nocturnal doses of MDMA may produce impairments of tracking performance and divided attention throughout the night that are additive to performance impairment produced by sleep loss. 
The study described in chapter $\mathbf{8}$ was designed to assess the effects of MDMA and alcohol, combined and alone, on actual driving performance and laboratory tasks related to driving. Eighteen recreational ecstasy users participated in a doubleblind, placebo-controlled, six-way cross-over study. Treatments consisted of MDMA 0, 75 and $100 \mathrm{mg}$ with and without alcohol, aiming at $0.06 \mathrm{mg} / \mathrm{ml} \mathrm{BAC.}$ Laboratory tests (Critical tracking task, Object movement estimation task) were conducted between 1.5 and 2 hours post-drug ( 0.5 and 1 hour post alcohol). Actual driving tests (Road tracking test, Car-following test) were conducted between 3 and 5 hours post-drug ( 2 and 4 hours post-alcohol). Subjects completed the Addiction research center inventory (ARCI) and rated their driving quality and mental effort during driving. It was shown that alcohol alone impaired critical tracking performance, as well as a number of actual driving performance parameters e.g. standard deviation of lateral position (SDLP), brake reaction time, coherence. MDMA alone reduced SDLP and standard deviation of speed. MDMA significantly moderated alcohol-induced impairment of road tracking performance but did not affect alcohol impairments of car-following and laboratory task performance. Subjective data seemed to support objective data. It was concluded that MDMA moderated the impairing effects of a low dose of alcohol on road tracking performance but nevertheless could not overcome alcohol-induced impairment on other aspects of driving behaviour or driving related performance.

In chapter 9 several topics related to MDMA use and MDMA research were reviewed. First, the selectivity of the by MDMA-induced memory impairment was discussed, followed by a review of MDMA's potential to be a neurotoxin. It was concluded that the memory impairment caused by a single, and a repeated dose of MDMA was selective because other tasks were not affected. Moreover, this impairment was temporary as it was no longer visible in the withdrawal period i.e. 25.5 hours post-drug. It can therefore be assumed that a single dose of ecstasy causes no persistent functional damage. Furthermore, the concordance of memory deficits in abstinent users and during intoxication let us suggest that a similar mechanism underlies this impairment. It was suggested that $5-\mathrm{HT}_{1 \mathrm{a}}$ agonism in combination with elevated levels of cortisol, and chronic stress may be contributory mechanisms. Future mechanistic studies can perhaps provide additional evidence for this notion.

In addition, it was suggested to include additional experimental factors (combinations of drugs, physical exertion) in future research to increase the ecological validity of controlled laboratory studies and consequently to gain more insight in the real-life situation. 
168 | SUMMARY 


\section{SAMENVATTING}

Ecstasy (MDMA) is een illegale drug die erg populair is bij mensen die vaak naar grote dansfeesten gaan. Een schatting van het recent drug gebruik bij Europese jongeren en jong volwassenen (15-34 jaar) laat zien dat tussen 0.6 en $6 \%$ van deze groep ecstasy gebruikt. Ondanks het wijdverbreide en frequente gebruik van de drug, is het aantal gevallen met acute medische problemen klein. Niettemin wordt er onrust gewekt door mogelijke lange termijn effecten van het gebruik van ecstasy op cognitieve functies zoals geheugen. Vele studies die de cognitieve prestaties van ecstasy gebruikers onderzochten hebben aangetoond dat huidige en ex-ecstasy gebruikers impulsiever zijn en een slechtere geheugenprestatie hebben in vergelijking met een referentie groep (bvb. polydrug gebruikers of cannabis gebruikers). Het probleem is echter dat deze verschillen in prestatie te wijten kunnen zijn aan vertekenende demografische variabelen zoals bijvoorbeeld een andere levensstijl of een premorbide niet-gediagnostiseerde psychopathologie. Bijgevolg blijft de vraag of de gerapporteerde cognitieve problemen veroorzaakt worden door ecstasy zelf of door andere factoren die gerelateerd zijn aan het gebruik van ecstasy. Het hoofddoel van de studies die gepresenteerd worden in dit proefschrift is om te onderzoeken of er een causaal verband bestaat tussen het gebruik van ecstasy en prestatiebeperkingen, en om het psychofarmacologische mechanisme te verduidelijken. Het experimentele onderzoeksdesign dat gebruikt wordt om dit te onderzoeken is een placebogecontroleerd, binnengroepsdesign. In de eerste vier hoofdstukken die volgen op de introductie worden 4 studies beschreven die de relatie tussen ecstasy (MDMA) en geheugenproblemen onderzoeken. De laatste drie experimentele hoofdstukken focussen op de relatie tussen MDMA en de mate van impulsiviteit en psychomotorisch functioneren.

Hoofdstuk 1 biedt een algemeen overzicht over wat ecstasy (MDMA) is, wat het doet in het lichaam, met welke cognitieve problemen het gekoppeld wordt, en de beperkingen van bestaande studies. Deze beperkingen worden besproken en een alternatief design, welk deze problemen omzeilt, wordt voorgesteld.

In hoofdstuk 2 worden de acute en residuele effecten van een enkele dosis MDMA (75 mg) op verbaal geheugen gepresenteerd. Achttien recreationele ecstasy gebruikers namen deel aan deze dubbel-blinde, placebo gecontroleerde, drieweg binnengroepsstudie. Er werd angetoond dat MDMA 1,5 uur na inname het geheugen verstoorde maar dat deze effecten de volgende dag, 25,5 uur na inname, 
verdwenen waren. De conclusie is dat de geheugenbeperkende effecten van een enkele dosis MDMA van voorbijgaande aard zijn.

Het doel van de in hoofdstuk 3 beschreven studie was in de eerste plaats om te kijken of een enkele dosis MDMA de spatiele geheugenprestatie aantast tijdens de intoxicatie fase en de ontwenningsfase, respectievelijk 1,5 uur en 25,5 uur na inname. De tweede doelstelling was om te onderzoeken of de spatiele geheugenprestatie na MDMA-inname taakafhankelijk is. Achttien recreationele ecstasy gebruikers namen deel aan deze dubbelblinde, placebo gecontroleerde drieweg binnengroepsstudie. Zij kregen placebo, 75 mg MDMA en $20 \mathrm{mg}$ methylfenidaat toegediend. Geheugentesten, twee spatiële geheugentaken met variërende moeilijkheid, werden afgenomen tussen 1,5 en 2 uur en tussen 25,5 en 26 uur na drug-inname. Bij de ene taak, de 'Spatial memory'-taak, diende enkel de locatie van de stimulus onthouden te worden; bij de andere taak, de 'Change blindness'-taak moest er eveneens inhoudelijke of contextuele informatie verwerkt worden. Resultaten toonden aan dat proefpersonen, 1,5 uur na inname van MDMA, bij de uitvoering van de 'Spatial memory'-taak grotere lokalisatiefouten makkten en sneller reageerden. Een regressieanalyse wees uit dat die onnauwkeurigheid niet te wijten was aan de verhoogde reactiesnelheid. Prestatie in de 'Change blindness'-taak werd niet beïnvloed door een dosis MDMA. Methylfenidaat had geen invloed op de prestatie van beide taken. De conclusie is dat een enkele dosis MDMA het spatiële geheugen voor locatie verslechtert en dat de verwerking van contextuele informatie gespaard blijft.

In hoofdstuk 4 wordt een studie beschreven met als doelstelling onderzoeken of de effecten van avonddossisen van MDMA op geheugen additief zijn aan de geheugenproblemen veroorzaakt door slaaptekort ofdat ze ermee interageren. Veertien recreationele ecstasy gebruikers namen deel aan deze dubbelblinde, placebo gecontroleerde, tweeweg binnengroepsstudie. Ze kregen $75 \mathrm{mg}$ en $50 \mathrm{mg}$ MDMA toegediend, respectievelijk om 20 en 24 uur, of tweemaal placebo. Geheugentesten en subjectieve metingen van gemoedstoestand werden afgenomen tijdens baseline (18u30) en op drie momenten na inname, namelijk om 21u30, 1u30 en $7 \mathrm{u}$, respectievelijk 1,5; 5,5 en 11 uur ten opzichte van drug-inname ( $1^{\mathrm{e}}$ dosis). De geheugenprestatie verslechterde progressief over de tijd als functie van slaaptekort, onafhankelijk van behandeling (MDMA/Placebo). Het hoofdeffect van MDMA op zowel verbaal als spatieel geheugen toont aan het effect van MDMA op geheugenprestatie additief was ten opzichte van het effect veroorzaakt door slaapdeprivatie. De stemmingsvragenlijst en de reactiesnelheid onthulden een 
interactie van MDMA en tijdstip van meting. $\mathrm{Na}$ toediening van MDMA verbeterden reactiesnelheid en gemoedstoestand/stemming; zo vergrootten gevoelens van vriendelijkheid, uitgelatenheid, 'levendigheid', angst, verwarring, arousal en positieve gemoedstoestand tijdens de nacht. Al deze parameters (inclusief reactiesnelheid) waren tijdens de ochtendsessie vergelijkbaar met deze na toediening van placebo. De conclusie is dat avonddosissen van MDMA selectief het geheugen verslechteren en dat deze negatieve invloed additief is ten opzichte van het effect van slaapdeprivatie op geheugen.

Hoofdstuk 5. De studie die beschreven wordt in dit hoofdstuk had als doel te onderzoeken welke neurale structuren aan de basis liggen van een verslechterde geheugenprestatie, veroorzaakt door een enkele dosis MDMA. Zeven gezonde recreationele ecstasy gebruikers (mannen) namen deel aan deze studie. De studie werd uitgevoerd volgens een dubbelblind, placebo gecontroleerd, binnengroepsdesign. Proefpersonen kregen $75 \mathrm{mg}$ MDMA en placebo toegediend Vóór drug-inname leerden proefpersonen een lijst van 15 woorden; dit is de controle lijst. Anderhalf uur na druginname werden de proefpersonen in een 3Telsa scanner geplaatst. Hier moesten ze een experimentele lijst met 15 nieuwe woorden lezen en leren; gevolgd door een presentatie van de controle lijst. Beide lijsten werden afwisselend elk vijf maal gepresenteerd en proefpersonen moesten na iedere presentatie zo veel mogelijk woorden van de lijst trachten te reproduceren. Gedragsmaten waren het aantal woorden, dat correct herinnerd werd na iedere presentatie en hun som over de 5 presentaties. Functionele data (scanner) bestonden uit registraties van het 'BOLD'-signaal tijdens de presentatie van beide lijsten. Resultaten toonden aan dat het leren van woorden hoofdzakelijk gerelateerd was met activiteit in frontale hersengebieden. Wanneer proefpersonen onder invloed waren van MDMA was de BOLD-response groter, onafhankelijk van lijst-type, in het linker mediaal frontaal gebied, welk gerelateerd is met het leren van woorden. Op gedragsniveau bleken geheugenscores lager te zijn wanneer proefpersonen onder invloed waren van MDMA; dit was echter niet statistisch significant. Er werd aangetoond dat een enkele dosis MDMA een verhoging in BOLD activiteit veroorzaakt tijdens het leren en/of lezen van de experimentele lijst en de controle lijst. Dit lijkt er op te wijzen dat het linker mediale frontale gebied, welk gerelateerd is met het leren van woorden, een rol speelt in de verslechterde geheugenprestatie die op gedragsniveau werd waargenomen. De voorlopige conclusie van deze studie is dat een enkele dosis MDMA het verbale geheugen verstoort door te interfereren met het woorden-leer-proces ofwel encodeer-fase. 
In hoofdstuk 6 worden de effecten MDMA, alleen en in combinatie met alcohol, op maten van impulsiviteit en risicogedrag gepresenteerd. Achttien recreationele ecstasy gebruikers namen deel aan deze dubbelblinde, placebo-gecontroleerde zesweg binnengroepsstudie. Ze kregen een enkele dosis MDMA (0 mg, 75 mg, 100 $\mathrm{mg}$ ) die al dan niet gecombineerd werd met een dosis alcohol. De alcoholdosering was zodaning dat er een piek bloedalcohol concentratie bereikt werd van ongeveer 0,06 g/dl tijdens de gedragsmeting. Metingen (Stop-signal taak, Go/no-go taak, Iowa gambling taak) werden tussen 1,5 en 2 uur na inname van MDMA uitgevoerd. MDMA veroorzaakte een daling in de stop reactietijd van de Stopsignal taak wat duidde op een verbeterde impulscontrole. Alcohol veroorzaakte een verhoging van de proportie commissiefouten in de Stop-signal taak en de Go/ nogo taak. Een signaal detectie analyse van deze parameter toonde aan dat de stijging in de foutenproportie een verstoring van perceptuele processen of aandachtsprocessen illustreert en niet zozeer een verhoging van motorische impulsiviteit. De prestatie van proefpersonen in de Iowa gambling taak werd niet beïnvloed door MDMA of alcohol maar er was een niet-significante trend die in de richting van een verbetering na inname van alcohol wees. Geen van de impulsiviteits-gedragsmaten vertoonde een interactie van MDMA en alcohol. De afwezigheid van deze interactie toonde aan dat de stimulerende effecten van MDMA nooit voldoende waren om de door alcohol-geïnduceerde verslechtering van impulscontrole en risicogedrag te overwinnen.

In hoofdstuk 7 wordt een studie beschreven die als doel had de effecten van nachtelijke dosissen van MDMA te onderzoeken op impulsiviteit en psychomotorische prestatie. Veertien recreationele ecstasy gebruikers namen deel aan deze dubbelblinde, placebo gecontroleerde, tweeweg binnengroepsstudie. Proefpersonen kregen $75 \mathrm{mg}$ en $50 \mathrm{mg}$ MDMA toegediend, respectievelijk om 20 en 24 uur, of tweemaal placebo. Gedragsmetingen werden viermaal tijdens de avond en nacht uitgevoerd namelijk om 18u30, 21u30, $1 \mathrm{u} 30$ en $7 \mathrm{u}$, respectievelijk 1,$5 ; 1,5 ; 5,5$ en 11 uur na inname van MDMA (1 ${ }^{\mathrm{e}}$ dosis). Een slaapschaal werd tijdens de avond en nacht, in totaal tien maal, gepresenteerd aan de proefpersonen. Resultaten toonden aan dat MDMA een verslechterende invloed uitoefende op de oog-handcoördinatie (tracking) in een simpele tracking taak. Verder werd er aangetoond dat prestatie op een verdeelde aandachtstaak negatief beïnvloed werd door MDMA; dit kon afgeleid worden uit de verslechterde prestatie op de secundaire tak. MDMA had geen invloed op motorische en cognitieve impulsiviteit. Prestatie op een vigilantie taak om 5 uur 's nachts liet zien dat de 
prestatie afnam in functie van tijd maar dat deze afname kleiner was wanneer proefpersonen onder invloed waren van MDMA, in vergelijking met placebo. Proefpersonen scoorden tijdens de nacht lager op de slaapschaal in vergelijking met placebo, maar in de ochtend was dit verschil weg en waren scores even hoog. De conclusie is dat nachtelijke dosissen van MDMA een verslechtering van tracking prestatie en verdeelde aandacht veroorzaakt die additief is aan prestatieverslechtering veroorzaakt door slaaptekort.

In hoofdstuk 8 worden de effecten van MDMA alleen, of in combinatie met alcohol, op psychomotorisch functioneren en rijvaardigheid beschreven. Achttien recreationele ecstasy gebruikers namen deel aan deze dubbelblinde, placebogecontroleerde, zesweg binnengroepsstudie. Ze kregen een enkele dosis MDMA (0 $\mathrm{mg}, 75 \mathrm{mg}, 100 \mathrm{mg}$ ) die al dan niet gecombineerd werd met een dosis alcohol. De alcoholdosering was zodaning dat er een piek bloedalcoho concentratie bereikt werd van ongeveer $0,06 \mathrm{~g} / \mathrm{dl}$ tijdens de gedragsmeting. Labmetingen (Tracking taak, Object movement estimation taak) werden tussen 1,5 en 2 uur na inname van MDMA, (tussen 0,5 en 1 uur post-alcohol) afgenomen. De rij-testen (Road tracking, Car-following) werden tussen 3 en 5 uur na inname uitgevoerd (tussen 2 en 4 uur post-alcohol). Proefpersonen vulden een vragenlijst in (ARCI) en beoordeelden na afloop van de autorit hun rij-prestatie en mentale inspanning tijdens het rijden. Resultaten wezen uit dat alcohol de tracking-prestatie negatief beïnvloedde, evenals een aantal rij-prestatie parameters bvb. de standaardafwijking van laterale positie (slingeren) en de rem-reactiesnelheid. MDMA veroorzaakte een vermindering van het slingeren en een verkleining van de standaardafwijking van snelheid. In combinatie met alcohol verminderde MDMA de verslechterende invloed van alcohol op een parameter van de road tracking taak; dit was echter niet het geval voor de parameters van de car-following taak, noch van de lab-metingen. Er wordt geconcludeerd dat MDMA de verslechterende invloed van een lage dosis alcohol op één parameter van de road tracking taak verminderde maar MDMA kon echter niet de door alcohol-geïnduceerde beperkingen op andere aspecten van rijgedrag of aan rijvaardigheid gerelateerd gedrag verminderen.

In hoofdstuk 9 worden verschillende onderwerpen gerelateerd aan MDMAgebruik en MDMA onderzoek besproken. Eerst wordt de selectiviteit van de geheugenverslechtering door MDMA besproken gevolgd door een overzicht van het neurotoxisch potentieel van MDMA. Er wordt geconcludeerd dat de geheugenverslechtering die veroorzaakt wordt door een enkele en herhaalde dosis van MDMA selectief is omdat andere cognitieve taken niet beïnvloed werden. 
Daarnaast was deze geheugenverslechtering tijdelijk en niet langer waarneembaar in de 'ontwenningsperiode', 25,5 uur na inname van MDMA. Het kan daarom aangenomen worden dat een enkele dosis van MDMA geen blijvende functionele schade veroorzaakt. Het werd gesuggereerd dat $5-\mathrm{HT}_{1 \mathrm{a}}$ agonisme in combinatie met verhoogde cortisollevels en chronische stress mechanismen zijn die bijdragen aan de geheugenverslechtering. Toekomstige mechanistische studies kunnen misschien bijkomend bewijs voor deze gedachte leveren.

Er werd ook nog voorgesteld om bijkomende experimentele factoren zoals combinaties van drugs en fysieke inspanningen in toekomstig onderzoek te includeren. Deze toevoeging van facoren zou de ecologische validiteit van gecontroleerde laboratoriumstudies vergroten en bijgevolg meer inzicht geven in de situatie zoals die in het dagelijkse leven voorkomt. 


\section{DANKWOORD}

In dit laatste deel van mijn 'boekje' wil ik even stilstaan en een woord van dank richten tot de mensen die een rol hebben gespeeld op persoonlijk en/of professioneel vlak deze afgelopen vier jaar waarin ik mijn promotieonderzoek heb afgerond. Vooralleer ik dit doe wil ik eerst en vooral even vertellen hoe het voor mij geweest is. De 4-jaren van mijn AiO-schap waren een ontdekkingsreis in de wondere wereld van de wetenschap. Ik ben tot het besef gekomen dat je enkel tot goede dingen kunt komen door te doen, en opnieuw te doen, te leren uit je eigen tekortkomingen, en te beseffen dat wetenschap het ontdekken van nieuwe dingen is, maar nog meer het ontdekken is van hoeveel er nog ontdekt moet worden. Maar uit elke ervaring kan geleerd worden en met die ingesteldheid kan het alleen maar beter gaan, zowel in het leven als in het werk. Ik heb mezelf weer een stukje beter leren kennen, ben mezelf soms tegengekomen maar ik ben bovenal gegroeid door de ervaring op professioneel alsook op persoonlijk vlak. Daarom ben ik ook zo blij dat ik dit alles heb kunnen ervaren.

Als eersten dank ik dan ook mijn promotor Wim Riedel en co-promotor Jan Ramaekers voor de kans die ik gekregen heb; voor het vertrouwen dat ze in mij hadden om zo een belangrijk project aan me toe te vertrouwen. Wim, we hebben niet zoveel contact gehad maar ik heb je commentaar op mijn werk in het laatste stadium van mijn loopbaan als $\mathrm{AiO}$ toch als interessant en bruikbaar ervaren. Jan, waar moet ik beginnen? Bedankt voor alles, bedankt dat je deur altijd voor me openstond. Je wist het altijd goed bij te sturen als het dreigde te ontsporen en had op die momenten telkens weer een pep-talk klaar. Je toonde interesse in dingen die speelden op persoonlijk en op professioneel vlak. Een betere begeleider kon ik me niet wensen!

Marleen, een collega zoals jij is goud waard! Ik heb genoten van de (bijna) vier jaar tijdens welke we een werkplek, maar ook lief en (AiO-)leed hebben gedeeld. Wat hebben we samen gelachen en plezier gemaakt; en het is een feit dat het zonder jou een stuk moeilijker was geweest! Ik apprecieerde je altijd luisterend oor en je oprechte interesse in mijn leven. Bedankt dat je mijn duidelijke aanwezigheid zo hebt kunnen verdragen. We verstaan elkaar met een woord. Je doet me beseffen dat werk leuk kan zijn, maar, dat dat tikkeltje meer wordt gemaakt door de mensen die je omringen.

Eef, bedankt dat je me hebt laten meegenieten van jouw ervaringen met onderzoek, cursussen en dergelijke. Je tips en informatie waren altijd handig en het heeft me vaak een hoop tijd bespaard! Anita, Tim, Peter, Anke, Arie, Arjan en 


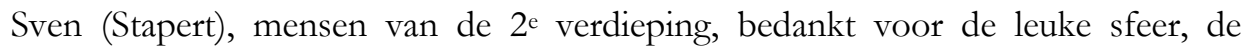
babbels, de fijne lunchpauzes, de leuke e-mails. Jullie zijn geweldige collega's! Sven (Gijsen), bedankt voor de technische assistentie tijdens het scannen en voor je eprime programmeertalent dat ik erg kon gebruiken! Henk, Irma, en Willy, bedankt logistieke ondersteuning tijdens de rijstudies en bedankt voor de data die jullie voor me verzameld hebben! Cees, bedankt voor de medische ondersteuning bij het onderzoek.

Verder wil ik mijn stagiaires Philippe, Mark, Kirsten, Lisa en Nina, bedanken; zonder jullie hulp had ik het niet gekund! Kirsten en Lisa, mijn twee opgewekte stagiaires en landgenotes, wat heb ik genoten van jullie aanwezigheid. Iedere dag dat jullie er waren was lachen! Jullie contact met de proefpersonen was schitterend en jullie doorzettingsvermogen geweldig. Nina, mijn laatste stagiaire tijdens mijn . "AiO-carriere", ik was heel blij met je als stagiaire, door jou heb ik niet alleen de zware testnachten met proefpersonen moeten doormaken! Je was een erg goede stagiaire.

Verder wil ik dan ook al de mensen bedanken die als proefpersoon hebben meegewerkt aan onze studies; zonder jullie waren er geen data om over te rapporteren! Bedankt voor jullie bijdrage aan mijn werk en de wetenschap.

Jo, Ellen en Kristel, bedankt voor de cafe-avondjes en Jo, de lunches op woensdag waren leuk en ontspannend, en dé gelegenheid om weer eens bij te praten. Bedankt voor jullie steun en luisterend oor.

Ma en pa, Ken en Lies, oma en bonne bedankt om er te zijn en voor me te zorgen, allen op jullie manier. Moeilijke momenten in mijn werk weerspiegelden zich als stillere momenten in jullie aanwezigheid. Aangezien iedereen nog met me praat neem ik aan dat jullie dat er wel bijnamen. Maar wiens pad gaat over rozen?

Jurgen, lieve schat, bedankt om er te zijn voor mij, voor de leuke motorritten, ontspannende weekendjes weg, vakanties of leuke uitstapjes op zondag. Het samen genieten van eten of een lekker glaasje wijn. Bedankt om naar me te luisteren als ik mijn frustraties over mijn werk uitte. Bedankt om te zijn wie je bent en om je warm hart voor mij.

Tot slot wil ik iedereen bedanken die ooit mijn pad gekruisd heeft en iets voor me betekend heeft op professioneel of persoonlijk gebied. Ik hoop dat al deze mensen me blijven steunen en ik zal hun hulp en vriendelijkheid altijd op prijs stellen. Dank voor alles. 


\section{CurRiculum Vitae}

Kim Kuypers werd op 14 maart 1980 in Hasselt (België) geboren. In 1997 behaalde zij het diploma wetenschappen-wiskunde (Onbevlekte ontvangenis, Tongeren). Daarna ging ze psychologie studeren en in december 2002 sloot ze haar studie psychologie aan de Universiteit Maastricht af met een stage bij de experimentele psychofarmacologie unit (EPU). Ze studeerde af in de biologische psychologie (neuropsychologie). In januari 2003 begon ze als assistent in opleiding aan haar promotieonderzoek naar de relatie tussen MDMA, cognitie en rijvaardigheid, bij de EPU, waar ze voorheen stage gelopen had. Momenteel zet ze haar werk voort bij de EPU als post-doc onderzoeker. 
178| Publications 


\section{Publications}

Kuypers, K. P. C., \& Ramaekers, J. G. (2007). Acute dose of MDMA (75 mg) impairs spatial memory for location but leaves contextual processing of visuospatial information unaffected. Psychopharmacology, 198(4), 557-563.

Kuypers, K. P. C., Samyn, N., \& Ramaekers, J. G. (2006). MDMA and alcohol effects, combined and alone, on objective and subjective measures of actual driving performance and psychomotor function. Psychopharmacology, 187(4), 467-475.

Ramaekers, J. G., \& Kuypers, K. P. C. (2006). Acute Effects of 3,4Methylenedioxymethamphetamine (MDMA) on Behavioral Measures of Impulsivity: Alone and in Combination with Alcohol. Neuropsychopharmacology official publication of the American College of Neuropsychopharmacology, 31(5), 1048-1055.

Ramaekers, J. G., Kuypers, K. P., \& Samyn, N. (2006). Stimulant effects of 3,4methylenedioxymethamphetamine (MDMA) $75 \mathrm{mg}$ and methylphenidate $20 \mathrm{mg}$ on actual driving during intoxication and withdrawal. Addiction Abingdon, England, 101(11), 1614-1621.

Theunissen, E. L., Jonkman, L. M., Kuypers, K. P., \& Ramaekers, J. G. (2006). A combined neurophysiological and behavioural study into the stimulating effects of fexofenadine on performance. Journal of psychopharmacology Oxford, England, 20(4), 496505.

Kuypers, K. P. C., \& Ramaekers, J. G. (2005). Transient memory impairment after acute dose of 75mg 3.4-Methylene-dioxymethamphetamine. Journal of psychopharmacology Oxford, England, 19(6), 633-639.

Papers in press

Kuypers, K. P. C., Wingen, M., Limbert, N., Samyn, N., \& Ramaekers, J. G. (2007). Acute effects of nocturnal doses of MDMA on measures of impulsivity and psychomotor performance throughout the night. Psychopharmacology (Berl), 10.1007/s00213-0060679-6 [doi].

Wingen, M., Kuypers, K. P. C., \& Ramaekers, J. G. (2006). Selective verbal and spatial memory impairment after 5-HT1a and 5-HT2a receptor blockade in healthy volunteers pre-treated with an SSRI. Journal of psychopharmacology Oxford, England, DOI, 0269881106072506v1.

Wingen, M., Kuypers, K. P. C., \& Ramaekers, J. G. (2006). The role of 5-HT1a and 5HT2a receptors in attention and motor control: a mechanistic study in healthy volunteers. Psychopharmacology, DOI, 10.1007/s00213-006-0614-x.

Paper submitted for publication

Kuypers, K.P.C., Wingen, M., \& Ramaekers, J.G. (submitted). Memory and mood during the night and in the morning after repeated evening doses of MDMA 


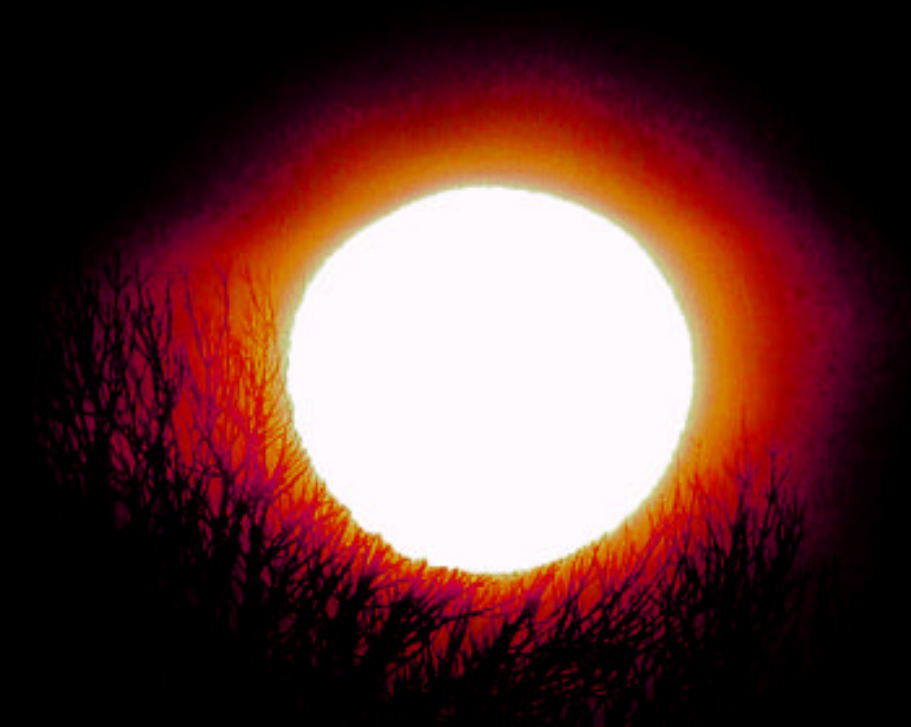

Ecstasy (MDMA) is an illegal drug, which is very popular among youngsters, frequently visiting large dance parties. Given the widespread and frequent use of ecstasy, the number of cases with acute medical problems is yet low. Nevertheless, serious concern has been raised by possible long-term effects of ecstasy use on cognitive functioning. Numerous studies investigating cognitive performance, e.g. memory, of ecstasy users have shown that ecstasy users have impaired memory performance and display elevated levels of impulsivity in comparison with a reference group. The problem however is that these differences in performance may be due to confounding demographic variables e.g. different lifestyle, premorbid undiagnosed psychopathology. Consequently the question remains whether the reported cognitive problems are caused by ecstasy itself or by other factors related to ecstasy use. The present thesis describes a range of studies that were designed to assess the effects of MDMA on memory, impulsivity and psychomotor performance during intoxication. The major aim was to establish a quantitative and causal association between MDMA exposure and performance impairment. 